\title{
Modeling neuropsychiatric phenotypes in mice in the frame of translational neuroscience
}

\section{Dissertation}

\author{
For the award of the degree \\ 'Doctor rerum naturalium' (Dr. rer. nat.) \\ of the Georg-August-University Göttingen
}

within the doctoral program Systems Neuroscience

of the Georg-August University School of Science (GAUSS)

submitted by

Martesa Tantra

from

Bogor, Indonesia

Göttingen, 2013 


\section{DOCTORAL THESIS COMMITTEE}

Prof. Dr. Dr. Hannelore Ehrenreich (Supervisor, First reviewer)

Clinical Neuroscience

Max Planck Institute of Experimental Medicine

Hermann-Rein-Straße 3

37075 Göttingen

Prof. Dr. Nils Brose (Second reviewer)

Department of Molecular Biology

Max Planck Institute of Experimental Medicine

Hermann-Rein-Straße 3

37075 Göttingen

Prof. Dr. Thomas Bayer

Division of Molecular Psychiatry

Department of Psychiatry

University of Göttingen

Von-Siebold-Straße 5

37075 Göttingen

Date of submission of thesis : Thursday, August $15^{\text {th }} 2013$

Date of oral examination

: Tuesday, September $17^{\text {th }} 2013$ 


\section{DECLARATION}

I hereby declare that this thesis has been written independently and with no other sources and aids than quoted.

Martesa Tantra

Göttingen, August $15^{\text {th }} 2013$ 


\section{ACKNOWLEDGEMENT}

The completion of my doctoral thesis has been a long and adventurous journey. I have encountered many amazing people with great talents, with whom I have made acquaintances and also friends. Most importantly, I have learned from each soul I've the honor to get to know. I have learned that life does not just stand still, nor wait until I have time to manage it. Much has happened and changed and I hope that I have evolved to be a better person. To these people, each in their own terms has contributed to my personal growth, I owe my gratitude. For without them, this doctoral thesis will not have been possible.

My deepest and utmost gratitude goes to my supervisor, Hannelore Ehrenreich, for giving me the opportunity to be a puzzle part of her wonderful research team. I sincerely thank you for your unlimited support and guidance along the way. Thank you for never being tired to discuss with me, teach me, and challenge me to make the best out of every little thing. I am grateful for every constructive critics and kind words every time, all those have convinced and motivated me and finally, brought me here.

I would further like to express my gratitude to Nils Brose and Thomas Bayer, as members of my thesis committee, for their supportive and encouraging discussions.

I would also like to extend my gratitude to Judith Stegmüller, Mikael Simons and Michael Müller, as members of my extended examination board, for taking the time and the effort to evaluate my work.

I am extremely grateful to Sonja Wojcik for the successful collaboration, from which the Baiap3 paper was born. It has been such a great pleasure to work with you!

To Ashraf Mannan and Chiranjeevi Bodda also I owe my gratitude, for the collaboration which not only granted me my first paper, but also new knowledge and perspectives and new acquaintances. I am sincerely thankful for their constant support and endless patience to answer to every question I had. 
I thank Kurt Hammerschimdt for his support and invaluable knowledge on ultrasound vocalization.

I wish to extend my special thanks to the past and present members of the "mouse profiler" team: Anja for her invaluable assistance and companionship; Daniela always for her optimism and kind gestures; Ekrem, Anes, Ahmed, Caro and Erin for the time we shared, the jokes we cracked; Jasmin and Aracely (my two little fairies) for their excellent assistance; Giulia and Pallavi for their endless support and willingness to do some gymnastic with my mice, and their enthusiasm for science which really is infectious, but of all, for their friendship. You guys rock!

Beata, Sergi, Artem, Kati, Mo, Karla and all past and present members of the Division of Clinical Neuroscience, to you All I owe a gigantic thank you, for every laughter, tears and every moment I spent here, it has nearly been a second home! I really treasure the opportunity to get to know you and to work with you all.

I sincerely thank Wiebke, for her never-ending patience to take care of many organizational issues, and of course, for her warm and cheerful attitude, especially in Monday mornings!

My special gratitude goes to the animal care takers, not only for taking care of my breeding mice, but also for taking care of me $;$; and making my time in the animal house much more enjoyable with their laughter and friendliness. Also to Rainer Libal, I owe many thanks for his support, kindness and patience.

I am sincerely grateful to the members of Feinmechanik and IT department and all workers of Max Planck Institute of Experimental Medicine, for their endless support and kindness and for making my time here is pleasantly memorable.

I would like to express my gratitude and appreciation for the team of GGNB and CSN offices for their excellent assistance on the administrative matters during my doctoral period.

My deepest gratitude to Linh and Dusan, for their endless long distance support and comforting voice over the phone every now and then. 
Swetlana, to you I am deeply grateful; for in you have I found a beautiful friendship, which shows me the true meaning of "a friend in need is a friend indeed".

Kostik, thank you for sharing with me so many of your Russian jokes and your food every so often. But not only that, also thank you for sharing your knowledge and passion about behavioral neuroscience and for keeping me reminded of the significance of insignificant things! I am very grateful for every kind word you say to lift me up at times and to remind me not to get ahead of myself $(;)$

I am deeply thankful for the continuous love of the Koop family, for their endless tolerance even when I am being rather intolerable and for believing in me.

To the Ms in my life, my safe haven, Mama and Markus. I do not know enough words to even begin to express my gratitude and how I feel... This achievement is for you.

Watch your thoughts, for they become your words Watch your words, for they become your actions Watch your actions, for they become your habits Watch your habits, for they become your character Watch your character, for it becomes your destiny 


\section{TABLE OF CONTENTS}

1. INTRODUCTION TO THE SCIENTIFIC PROJECT 1

The complexity of neuropsychiatric disorders: comorbidity is common, but causality is difficult to determine

Dysregulation of the transcriptional regulator, MECP2 is attributable to a wide spectrum of neuropsychiatric phenotypes

Mice as a model system to study complex human disorders $\quad 7$

2. FOCUS OF THE PRESENT WORK 9

a. Aims of project I 9

b. Aims of project II 10

C. Aims of project III 10

3. MOUSE MODEL TARGETING BAIAP3 GENE: 11

Genetic markers of a Munc13 protein family member, BAIAP3, are gender-specifically associated with anxiety and benzodiazepine abuse in mouse and man
a. Overview of project I
11
b. Original publication

4. MOUSE MODEL TARGETING A TRANSCRIPTIONAL REGULATOR, MECP2 (I):

Mild overexpression of Mecp2 in mice causes a higher susceptibility toward seizures
a. Overview of project II
b. Original publication

5. MOUSE MODEL TARGETING A TRANSCRIPTIONAL REGULATOR, MECP2 (II)

Mild expression differences of MECP2 influencing aggressive social behavior
a. Overview of project III
b. Submitted manuscript

6. DISCUSSIONS AND SUMMARY 110

$\begin{array}{lll}\text { 7. REFERENCES } & 115\end{array}$

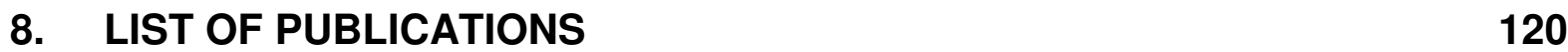

$\begin{array}{lll}\text { 9. } & \text { APPENDIX } & 121\end{array}$

10. CURRICULUM VITAE 144 


\section{INTRODUCTION TO THE SCIENTIFIC PROJECT}

The present cumulative thesis has been prepared on the basis of a translational neuroscience approach based on schizophrenic patients of the GRAS (Göttingen Research Association for Schizophrenia) data collection (Ribbe, Friedrichs et al. 2010): translating the findings from basic research using animal models back to human, and vice versa. The thesis includes three original publications that focus on the establishment of novel genetic mouse models of neuropsychiatric phenotypes. The first paper identifies BAIAP3/Baiap3 as a genetic factor that modulates anxiety and the response to benzodiazepines in mouse and man (Wojcik, Tantra, Stepniak et al. 2013). The second paper focuses on an elevated seizure propensity as the phenotypical consequences of mildly overexpressing the transcriptional regulator Mecp2 at $\sim 1.5$ fold wild-type level (Bodda, Tantra et al. 2013). The third publication expanding the results published in the second paper, now reveals that subtle alterations in the expression level of Mecp2/MECP2 influence social, particularly aggressive behavior (Tantra, Hammer, Kästner et al. submitted).

\section{The complexity of neuropsychiatric disorders: comorbidity is common, but causality is difficult to determine}

Neuropsychiatric disorders, such as autism spectrum disorders (ASD), schizophrenia, anxiety disorders, major depression and substance use disorders are highly prevalent (Kessler Rc 2005) and contribute substantially to disease burden worldwide. Neuropsychiatric disorders are complex traits that result from multiple genetic determinants interacting in the context of poorly understood environmental factors to trigger the emergence of clinically diverse phenotypes (Cristino, Williams et al. 2013). Family and twin studies have revealed that genetic factors play a major role in psychiatric disorders. For example, twin studies showed that heritability for schizophrenia is estimated to be $\sim 80 \%$ (Kas, Fernandes et al. 2006), while concordance between monozygotic twins is as high as 90\% for ASD (Abrahams and Geschwind 2008). Many putative genetic factors that confer risk to these disorders have been identified in individuals from diverse population, but the fact that affected individuals harbor different risk alleles in a heterogeneous genetic background still make candidate disorder genes difficult to detect (Cristino, Williams et al. 2013). 
However, despite large research efforts, the exact neurobiological mechanisms of neuropsychiatric disorders remain largely unknown.

Decades of research in psychiatry have revealed that anxiety disorders and substance use disorders (SUDs) are commonly found in a comorbid state at greater rates than would be expected by chance alone (Smith and Book 2008). Anxiety disorders, with lifetime prevalence of $28.8 \%$ and SUDs, with lifetime prevalence of $14.6 \%$ are among the most frequent psychiatric problems (Kessler Rc 2005). Recent analyses from an epidemiological survey on more than 40.000 individuals (the National Epidemiologic Survey on Alcohol and Related Conditions, USA) revealed striking rates of co-occuring anxiety and SUD: anxiety disorders were significantly related to alcohol and drug use disorders (Compton Wm 2007; Smith and Book 2008). When anxiety disorders co-occur with SUDs, there is a clinical impact since these disorders are functionally intertwined in both the development and maintenance of the comorbidity (Smith and Book 2008). The pathways to comorbidity of anxiety disorders and SUDs can vary (Smith and Book 2008):

(1) a self-medication pathway wherein an anxiety disorder leads to a substance use disorder (i.e. to alleviate the symptoms of anxiety, a person selfmedicates, which in turn leads to SUD)

(2) a substance-induced anxiety disorder pathway (i.e. the consumption of a substance like alcohol, cocaine leads to intoxication which induces anxiety)

(3) a third variable pathway (e.g. genetic predisposition to develop either anxiety disorder and/ or substance abuse)

Alternatively, genetic factors might already play a role in modulating the above mentioned pathways (figure 1). The heritability for anxiety disorders is estimated to be 20-40\% (Hettema, Prescott et al. 2005) and 40-70\% for SUD, respectively (Ducci and Goldman 2012). This implies that individuals are differentially vulnerable to anxiety disorders and SUD. If an individual carries a genetic load to develop anxiety disorders, this individual will likely possess the behavioral traits that predispose it to develop drug-seeking behavior (DeMartini and Carey 2011; Pasche 2012) as a consequence of self-medication to alleviate the anxiety symptoms, which increases the risk of anxiety disorder and SUD co-occurs. Similarly, the converse might also be true. For an individual who carries the genetic predisposition for drug-seeking behavior, the odds to develop substance-induced anxiety will increase, contributing to the 
comorbidity of SUD and anxiety disorders. However, it is also very likely that there are common factors - both genetic and environmental - which interact with each other giving raise to the comorbidity of SUD and anxiety disorders.

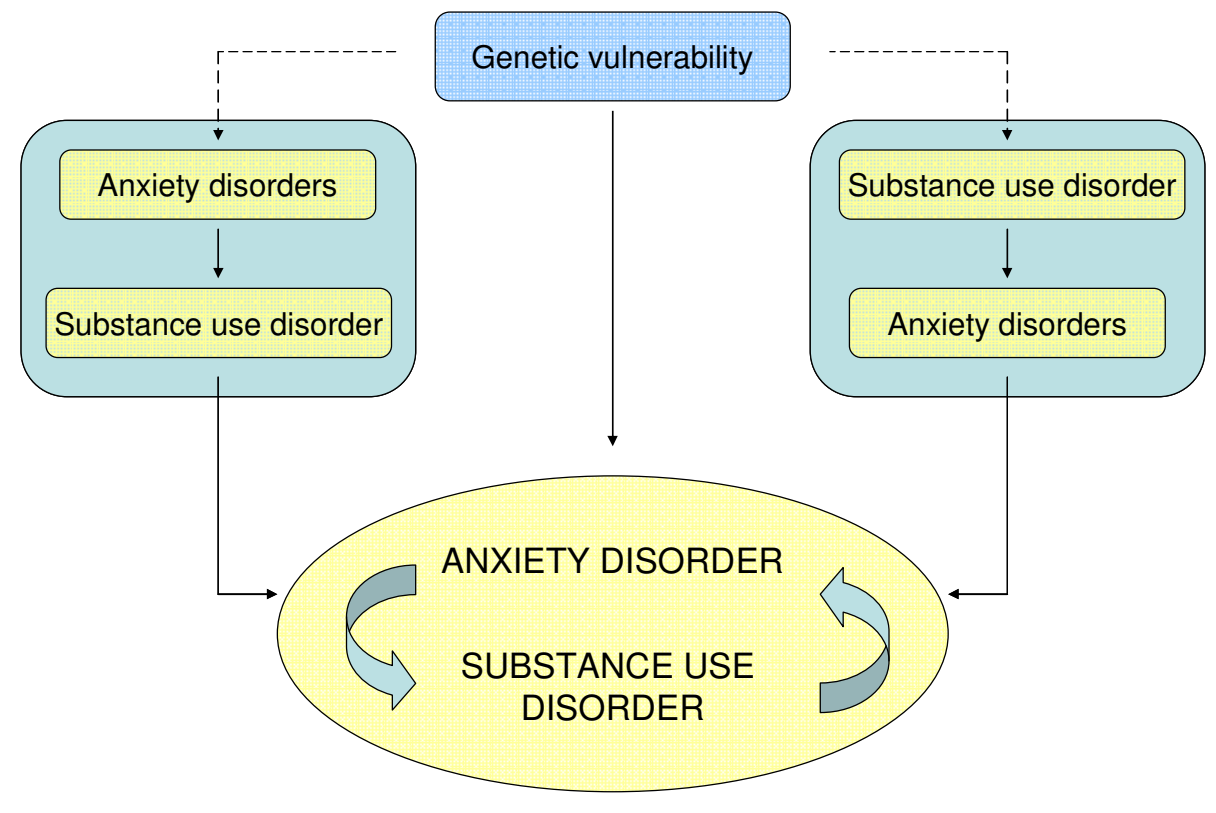

Modified from (Smith and Book 2008)

Figure 1. Development and mutual maintenance of comorbid anxiety disorder and SUD can happen through several pathways.

Although there have been a wide range of studies on finding candidate genes associated with addiction and anxiety disorders (Bierut, Madden et al. 2007; Gratacos, Sahun et al. 2007; Hamilton 2009; Al-Eitan, Jaradat et al. 2012; Ducci and Goldman 2012; Guo, Liu et al. 2012; Harari, Wang et al. 2012), our understanding of the etiology of these disorders are still limited, due to the role of environmental risk factors which also contribute to the emergence of the disorders. Thus, finding common factors underlying both disorders will help elucidating the pathways to the emergence of the disorders, which in turn will be valuable for future treatments for comorbid anxiety and SUDs. 


\section{Dysrequlation of the transcriptional requlator, MECP2 is attributable to a wide spectrum of neuropsychiatric phenotypes}

The transcriptional regulator MECP2 gene encodes for methyl-CpG-binding protein2 (MECP2), which binds to methylated CpG DNA sequences and modulates transcription of genes in the vicinity of methylated sequences (Lewis, Meehan et al. 1992; Nan, Ng et al. 1998; Amir, Van den Veyver et al. 1999; Chahrour and Zoghbi 2007; Chahrour, Jung et al. 2008; Guy, Cheval et al. 2011). Initially, MECP2 was thought to act as a transcriptional regulator, through the interaction with corepressor Sin3a and HDAC (Jones, Veenstra et al. 1998; Nan, Ng et al. 1998; Chahrour and Zoghbi 2007). Upon binding to methylated CpG islands, MECP2 recruits HDAC at the methylation loci which leads to the alteration of the chromatin, making it compact and thus, inaccessible to the transcription machinery (Jones, Veenstra et al. 1998; Nan, $\mathrm{Ng}$ et al. 1998; Chahrour and Zoghbi 2007). However, recent studies have elucidated another role of MECP2, as a transcriptional activator (Chahrour, Jung et al. 2008). MECP2 was shown to be associated with actively transcribed genes (Chahrour, Jung et al. 2008; Mellén, Ayata et al. 2012). Association between MECP2 and the transcriptional activator CREB1 was found on an active, but not repressed form of the same gene (Yasui, Peddada et al. 2007; Chahrour, Jung et al. 2008). Besides its role as a transcriptional regulator, MECP2 was also found to be involved in RNA splicing (Chahrour, Jung et al. 2008).

The MECP2 gene is $\mathrm{X}$-linked and thereby a subject to $\mathrm{X}$-inactivation during dosage compensation in females (Adler, Quaderi et al. 1995). This phenomenon is crucial, as it leads to different outcomes in individuals with MECP2 mutations, depending on gender, with males being more severely affected than females owing to hemizygosity (Guy, Cheval et al. 2011). MECP2 mutations are the cause of Rett syndrome (RTT), a neurological disorder affecting mainly girls during early childhood (Amir, Van den Veyver et al. 1999; Bienvenu, Carrie et al. 2000; Lee, Wan et al. 2001), with an incidence of $\sim 1 / 10000$ female live births (Chahrour and Zoghbi 2007). RTT is classically characterized by a period of normal development milestones until $\sim 6-18$ months of age, followed by loss of acquired cognitive, motor and social skills (Chahrour and Zoghbi 2007). The occurrence of epilepsy - from easily controlled to intractable epilepsy, with the most common types being partial and tonic-clonic seizures, is also a distinct feature of the disorder (Jian, Nagarajan et al. 2006; 
Chahrour and Zoghbi 2007). Also, symptoms of autism belong to the behavioral features of Rett syndrome (Mount, Charman et al. 2003; Moretti and Zoghbi 2006). Rett syndrome and autism have been recognized to have common clinical manifestations: RTT patients were regarded as an autism subgroup before Rett syndrome was recognized as a distinct disorder (Mount, Charman et al. 2003).

The discovery that loss-of-functions mutations in MECP2 cause the neurological disorder termed Rett syndrome, has led to the identification of other neuropsychiatric phenotypes caused by MECP2 mutations. Until now, more than 100 cases of copy number gains of the MECP2 gene have been reported (Ramocki, Tavyev et al. 2010; Breman, Ramocki et al. 2011; Van Esch 2012), causing a neurological disorder, termed as MECP2 duplication syndrome with symptoms overlap with Rett syndrome (Moretti and Zoghbi 2006; Van Esch 2012). Autism is common in boys with duplications spanning MECP2, and anxiety is a comorbid condition (Ramocki, Peters et al. 2009; Ramocki, Tavyev et al. 2010). MECP2 duplication syndrome is characterized by symptoms such as infantile hypotonia, severe mental retardation, poor speech development, progressive spasticity, ataxia and seizures (Ramocki, Tavyev et al. 2010; Van Esch 2012). A more severe phenotype is observed in individuals with triplications spanning MECP2 (del Gaudio, Fang et al. 2006).

The clinical picture of MECP2 mutations points to the necessity of a tight regulation of gene expression to ensure for proper function. Similar results were also obtained from studies on variety of mouse models of Mecp2 dysfunctions (Chen, Akbarian et al. 2001; Guy, Hendrich et al. 2001; Shahbazian, Young et al. 2002; Collins, Levenson et al. 2004; Guy, Gan et al. 2007; Samaco, Fryer et al. 2008; Goffin, Allen et al. 2012). Mice carrying Mecp2 mutations which lead to loss-of-functions displayed features similar to Rett syndrome, including neurological phenotypes, tremors, motor dysfunctions, seizures and breathing abnormalities (Table 1), with the severity of the phenotypes varies depends on the amount of functional Mecp2. 


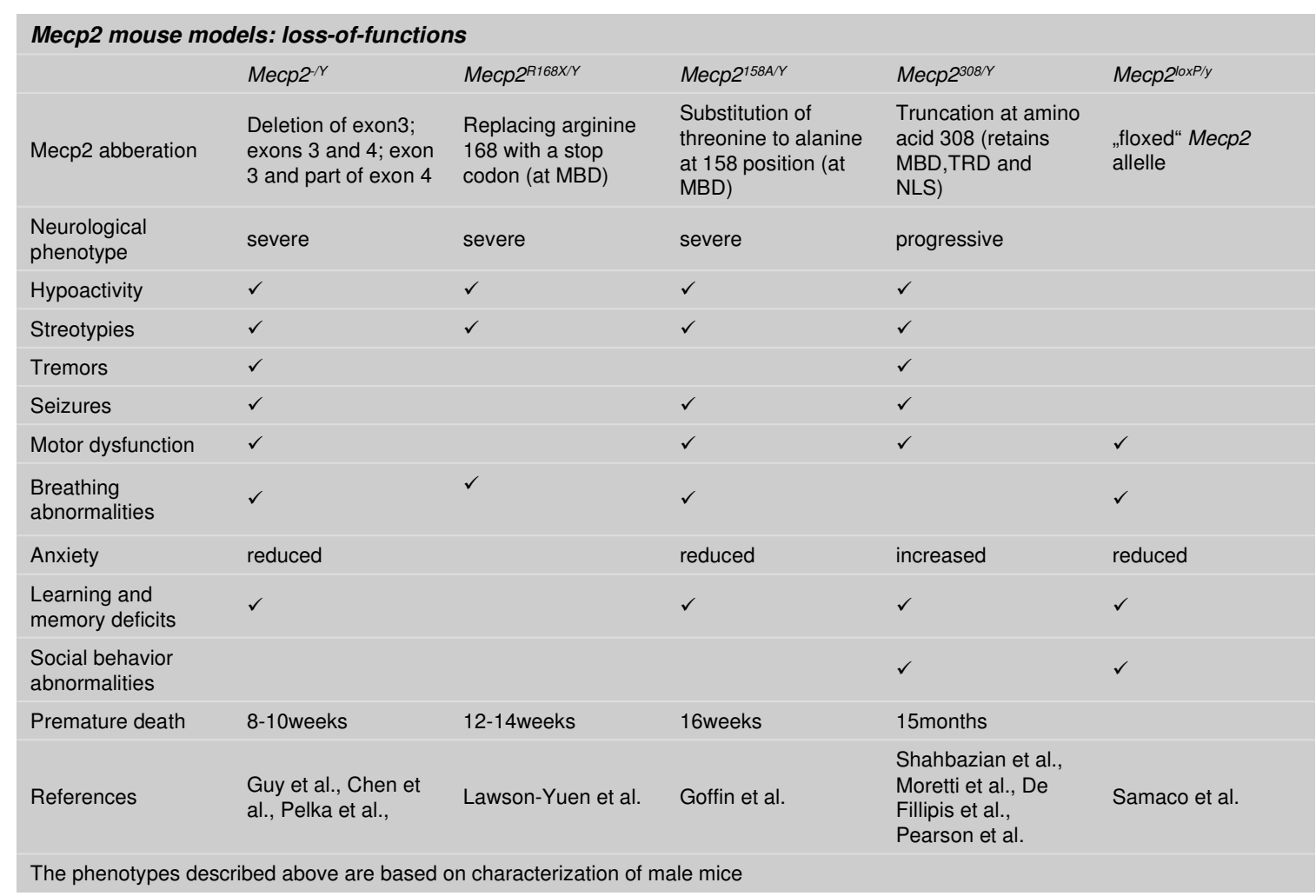

Adapted from (Chahrour and Zoghbi 2007)

Table 1. Summary of phenotypes induced by alterations of Mecp2 level in mouse models of Mecp2 mutations.

Additionally, other mouse models carrying Mecp2 mutations at specifically targeted regions have been characterized, for example mutants with deletion of Mecp2 in Sim1-expressing neurons (hypothalamus), aminergic neurons and GABAergic neurons (Fyffe, Neul et al. 2008; Samaco, Mandel-Brehm et al. 2009; Chao, Chen et al. 2010). These mutant mice also displayed features of Rett syndrome.

In contrast to mouse models of Mecp2 loss-of-functions, the study on Mecp2 overexpression mouse model is just at the beginning. Previously, a transgenic Mecp2 mouse (Mecp2-Tg1) was generated by overexpressing wild-type human protein using a large genomic clone containing the entire human MECP2 locus, resulting in Mecp2 expression at $\sim 2$ fold wild-type level (Collins, Levenson et al. 2004). The phenotypes of these mice are reminiscent of the human MECP2 duplication syndrome. The transgenic mice appeared normal until the age of 10-12 weeks; soon afterwards they develop progressive neurological symptoms which include stereotyped movements, motor dysfunction, epilepsy, hypoactivity, ataxia, kyphosis, followed by premature death (Collins, Levenson et al. 2004). Also, it was reported that increasing the copy numbers of Mecp2 in the transgenic mice which results in higher Mecp2 level, caused 
even more severe phenotypes (Collins, Levenson et al. 2004). Overall, it is evident that the level of Mecp2 is critical to ensure proper function, as both loss- and gain-offunction cause neurological phenotypes (figure 2) (Chao and Zoghbi 2012).

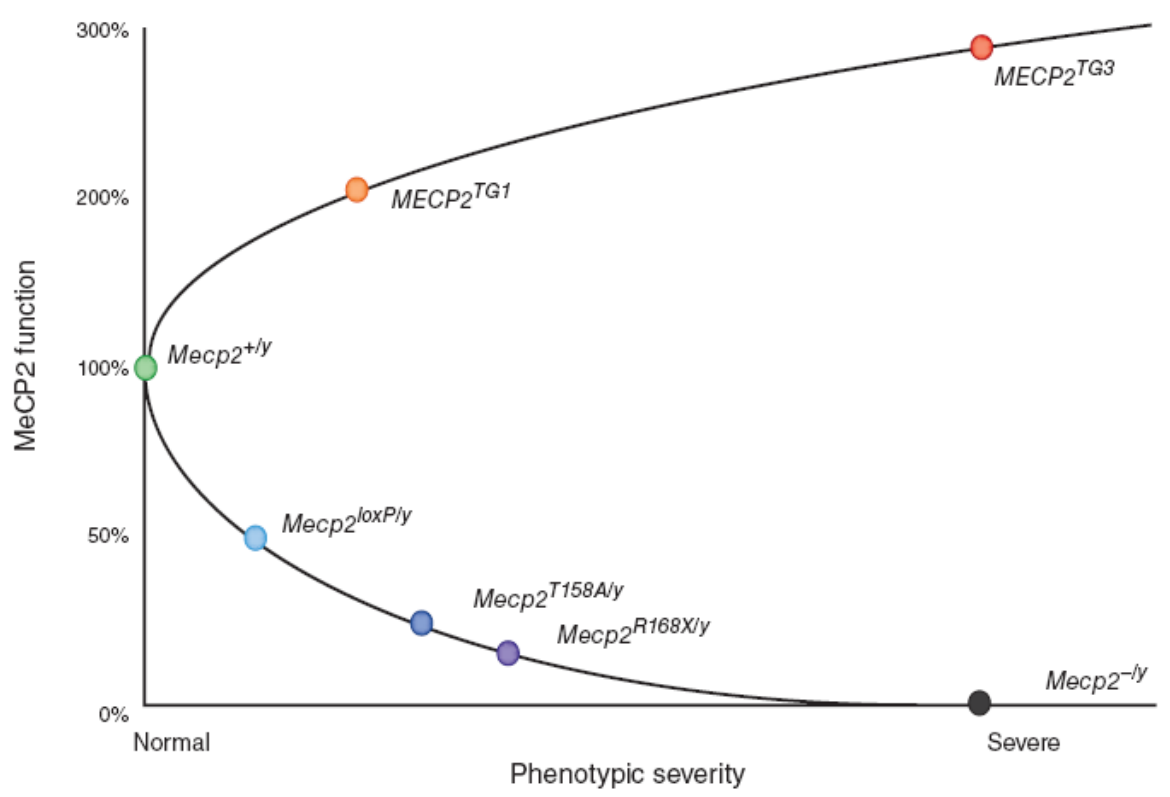

From (Chao and Zoghbi 2012)

Figure 2. Phenotypic severity of mutant Mecp2 increases as Mecp2 function decreases. Severity also increases however, as Mecp2 increases beyond normal $(100 \%)$ level.

\section{Mice as a model system to study complex human disorders}

Mice as a model system play an important role in elucidating the molecular pathways underlying such complex behavioral disorders. The similarity of the mouse and human genomes is remarkable, with most genes of one species occurring in the other, arranged in haplotypes of DNA that share many of the same genes in the same order in both species (Waterston, Lindblad-Toh et al. 2002). In fact, a working draft of the mouse genome has led to estimates that a mouse version may be identified for $99 \%$ of human genes (Tecott 2003). In accordance with their genomic homologies, humans and mice share numerous features of brain organization and may therefore exhibit similar behavioral responses to many pharmacological agents (Tecott 2003). Moreover, transgenic technology has allowed specific human alleles to be inserted into the mouse genome to create remarkable models for research (Wahlsten 2011). In 
the frame of complex human behavioral disorders, since the introduction of gene targeting techniques, diverse mutant mice carrying mutations of candidate genes of psychiatric disorders have been generated. Some of these mutant mouse strains were found to exhibit behavioral abnormalities reminiscent of human psychiatric disorders (Shahbazian, Young et al. 2002; Collins, Levenson et al. 2004; Jamain, Radyushkin et al. 2008; Radyushkin, Hammerschmidt et al. 2009; El-Kordi, Winkler et al. 2012).

In the context of modeling complex human behavioral disorders in mice, the study of animal behaviors as the final output of the nervous system is relevant because the behavioral traits/ phenotypes observed are: (1) influenced by multiple genes, (2) responsive to environmental influences and (3) normally distributed across the range of phenotypic expression (Bazzett 2008). Moreover, the use of mice in behavioral neuroscience has a long and illustrious history, containing well-validated and carefully controlled methods for behavioral testing of sensory abilities, motor functions, learning memory, social behavior, drug self-administration and traits relevant to neuropsychiatric disorders (Crawley 2007).

In my thesis, I will address a concept which focuses on the genetics of behavioral domains relevant to mouse behavior in the frame of human psychiatric disorders: abnormal social behavior associated with Mecp2 overexpression and Baiap3 deletion associated with anxiety-like phenotypes accompanied by an altered response to benzodiazepines. It will be shown in my thesis that interspecies trait genetics, which were assed by (1) comprehensive behavioral studies of genetic mouse models to find affected behavioral domains and (2) a phenotype-based genetic association study (PGAS) using the GRAS database, will optimize genotype-phenotype relationships for psychiatric disorders and facilitate the identification of biological substrates underlying these complex behavioral disorders. 


\section{FOCUS OF THE PRESENT WORK}

This cumulative thesis has been prepared in the frame of a translational neuroscience effort, applying studies from mice to men and vice versa, to delineate the biological basis of complex human behavioral disorders within a schizophrenic (GRAS, Göttingen Research Association for Schizophrenia) population. As part of a translational research group with a multidisciplinary character, my scientific work has been committed to the establishment of novel genetic mouse models of neuropsychiatric conditions. Working bi-directionally, the results from comprehensive behavioral characterizations of mutant mice provide the basis for further identifications of specific genotype-phenotype relationships using a phenotype-based genetic association study (PGAS), or vice versa. Presented in the thesis are 3 original first-authorship publications that investigate (1) the role of a member of the Munc13 family - synaptic regulators of neurotransmitter exocytosis - in modulating behavioral phenotypes relevant for human psychiatric disorders and (2) the consequences of subtle alterations in expression level of a transcriptional regulator MECP2. A forth publication which I co-author has been accepted for publication in Molecular Psychiatry. The manuscript is given in the appendix.

\section{a. Aims of project I}

The focus of the first study was to investigate the role of Baiap3/BAIAP3, which is highly expressed in brain regions involved in fear processing, and therefore possibly in mediating behavioral phenotypes relevant for human psychiatric disorders (Wojcik SM, Tantra M and Stepniak B et al. 2013).

To identify the role of Baiap3, a basic behavioral characterization of Baiap3 deficient mice ${ }^{1}$ and their wild-type (WT) littermates was performed. Subsequently, further behavioral experiments were designed to target specific phenotypes related to Baiap3 expression patterns: anxiety-like phenotypes and benzodiazepines dependence, tolerance and withdrawal. In parallel, in vitro analyses were performed to investigate whether lack of Baiap3 leads to alterations in neuronal activity. Additionally, the association between genetic variations of human BAIAP3 and behavioral phenotypes (anxiety and benzodiapine use disorder) was studied in the GRAS population.

\footnotetext{
${ }^{1}$ Baiap3 deficient mice were generated by Dr. Sonja M. Wojcik (Max Planck Institute of Experimental Medicine, Göttingen)
} 


\section{b. Aims of project II}

The second paper focused on identifying the earliest evidence of pathologies as consequences of gain-of-function properties of Mecp2, elicited by mildly overexpressing Mecp2 (Bodda C and Tantra M et al. 2013).

To evaluate the Mecp2 gene dosage effect, a transgenic mouse model of Mecp2 overexpression on $\mathrm{FVB} / \mathrm{N}$ genetic background was generated, resulting in transgenic mice with an overexpression of Mecp2 at $\sim 1.5$ fold wild-type (WT) level ${ }^{2}$. A comprehensive behavioral characterization of the male transgenic and WT mice was performed, with repetition over several cohorts, during early postnatal (neonatal) period and adulthood. The effect of Mecp2 overexpression on neuronal maturation and characterization and calcium homeostasis was also evaluated in vivo.

\section{c. Aims of project III}

In the third study, expanding the results of the second project, we aimed to explore behavioral consequences of mildly increased Mecp2/MECP2 expression, both in mice and humans (Tantra M, Hammer C, Kästner A et al. submitted).

First, using the Mecp2 transgenic construct expressed in two different mouse strains (FVB/N and C57BL/6N), we investigated behavioral domains, such as social behavior, which were targeted by Mecp2 overexpression. Furthermore, it was also our aim to translate the findings from mice back to humans, by investigating whether naturally occurring genetic variants such as single nucleotide polymorphisms (SNPs) of the MECP2 gene have an influence on social behavior. Putting the data obtained from the mouse and the human study in a context, we strive for mechanistic insights looking at SNP-related MECP2 expression differences, possibly due to miRNA regulations.

\footnotetext{
${ }^{2}$ The transgenic Mecp 2 mice were generated by PD Dr. Ashraf Mannan (Institute of Human Genetics, University Medical Center, Göttingen)
} 


\section{MOUSE MODEL TARGETING BAIAP3 GENE:}

\section{Genetic markers of a Munc13 protein family member, $B A I A P 3$, are gender-specifically associated with anxiety and benzodiazepine abuse in mouse and man}

\section{a. Overview of project I}

Diagnoses of psychiatric disorders rely heavily on the presence of a cluster of behavioral symptoms and their time course and a threshold beyond which the symptoms are considered to be clinically relevant, rather than the presence of any biological markers. Moreover, the observable symptoms are very much unlikely to be unique for a specific disorder. Potential problems with the process of establishing the diagnoses increase further when substance use disorders (SUDs) and psychiatric syndromes occur together (Schuckit 2006).

Anxiety disorders and SUDs, including benzodiazepine use disorder, are frequently comorbid (Smith and Book 2008). The interaction between anxiety disorders and SUDs is likely bi-directional and varies by the type of anxiety (Marmorstein 2012) and genetically determined anxiousness personality traits may predispose an individual more to develop an addiction (DeMartini and Carey 2011; Pasche 2012). Furthermore, the comorbidity of anxiety disorders and SUDs might reduce the treatment efficacy for either disorder. Benzodiazepines, which rank among the most frequently abused prescription medications (National Institute on Drug Abuse [http://www.nida.nih.gov]), are prescribed often to people with substance abuse problems (Mueller, Pagano et al. 2005) and also are still used as leading treatment of anxiety disorders (Clocis and Ferreira 2009; Baldwin, Allgulander et al. 2012). Benzodiazepines are advantageous treatments for anxiety disorders because they work quickly to alleviate the symptoms (Clocis and Ferreira 2009), however, there are concerns regarding their abuse liability, withdrawal reactions and development of tolerance (Argyropoulos and Nutt 1999). For these reasons, individuals with anxiety disorders with an existing comorbid benzodiazepine use disorder or a genetic liability for benzodiazepine use disorder may be at risk of adverse treatment outcomes. The identification of genetic predictors for anxiety disorders, and especially for benzodiazepine use disorder, could aid the selection of the best treatment option and improve clinical outcomes. In addition to 
human genetic association studies, animal models are used to study the genetic and neural circuitries of anxiety disorders and addiction. The selection of candidate genes targeted in animal studies is driven by hypotheses of neural circuitries and neurotransmitter systems which thought to be involved in mediating fear and anxieties (Gratacos, Sahun et al. 2007). Using a candidate gene approach, we investigated the involvement of the brain specific angiogenesis inhibitor l-associated protein 3 (Baiap3), which is highly expressed in brain regions involved in processing fear, such as the amygdala, hypothalamus and periaqueductal gray, and therefore in behavioral phenotypes relevant for human anxiety disorders.

Baiap3 is a member of the mammalian uncoordinated 13 (Munc13) family of synaptic regulators of neurotransmitter exocytosis (Shiratsuchi, Oda et al. 1998; Koch, Hofmann et al. 2000). The cellular function of Baiap3 is currently unknown, however, all other Munc13s are regulators of vesicle exocytosis in various cell types (Wojcik and Brose 2007). To uncover the function of Baiap3, we combined the behavioral analyses of Baiap3 null-mutant (KO) mice with a phenotype-based genetic association study (PGAS) of human BAIAP3 in a schizophrenic (GRAS) population.

The behavioral characterization of Baiap3 mice revealed that deletion of Baiap3 led to enhanced seizure propensity in mice of both genders and increased anxiety, a phenotype which was more pronounced in female mice. Accordingly, we hypothesized that genetic variation in human BAIAP3 may also be associated with anxiety. Two human BAIAP3 single nucleotide polymorphisms (SNPs) risk genotypes (AA for rs2235632 and TT for rs1132358) were identified. Both risk genotypes showed a significant association with anxiety in women and, surprisingly, with benzodiazepine abuse in men. Translating the findings of PGAS back to mice, we established an experimental paradigm of chronic benzodiazepine administration to investigate the development of dependence, tolerance and withdrawal to benzodiazepines in Baiap3 KO and WT mice of both genders. We found that male, but not female Baiap3 KO mice develop tolerance to diazepam more quickly than control mice. Furthermore, to assess whether Baiap3 KO mice would also be more likely to orally self-administer benzodiazepine, we performed an experiment of chronic self-administration of midazolam (a short-acting benzodiazepine). Here, no genotypedependent differences were observed, indicating that Baiap3 deficiency affected the development of tolerance and dependence to benzodiazepines at an early stage, 
prior to the development of addiction. One hypothesis regarding predisposition to the development of addiction is an altered response to addictive substance and its withdrawal at the cellular level. Subsequently, the response to diazepam treatment and withdrawal in hypothalamus neurons in vitro was studied. The results revealed a lack of homeostatic adaptation to diazepam treatment and withdrawal in Baiap3 KO hypothalamus slices. In summary, the first project of my thesis delivers evidence for Baiap3/BAIAP3 as a common genetic factor underlying anxiety and benzodiazepine abuse in mice and human.

\section{b. Original publication}

Wojcik $\mathrm{SM}^{\star}$, Tantra $\mathbf{M}^{*}$, Stepniak $\mathrm{B}^{*}$, Man KM, Müller-Ribbe K, Begemann M, Ju A, Papiol S, Ronnenberg A, Gurvich A, Shin Y, Augustin I, Brose N, Ehrenreich H (2013): Genetic Markers of a Munc13 Protein Family Member, BAIAP3, Are GenderSpecifically Associated with Anxiety and Benzodiazepine Abuse in Mouse and Man. Molecular Medicine

${ }^{*}$ Equal contribution

\section{Own contribution:}

I was responsible for conducting and analyzing all behavioral experiments in several different cohorts of Baiap3 KO mice: behavioral characterization, the investigation of benzodiazepine tolerance, dependence and withdrawal as well as the establishment of oral self administration of midazolam in Baiap3 KO and WT mice. I also performed the behavioral data analysis and prepared the figures for the manuscript (see Figure 2 and 3, Supplementary figure S3 and S6). Furthermore, I participated in the conception, design, drafting, revision and publication of the manuscript. Under the supervision of Prof. Hannelore Ehrenreich, I wrote and cited appropriately the materials and methods of the animal experiments sections of the manuscript. I also wrote the results section and the figure legends, designed the layouts of the figures of the results section and delivered the interpretations of the results for the discussion section of the manuscript. 


\title{
Genetic Markers of a Munc13 Protein Family Member, BAIAP3, Are Gender Specifically Associated with Anxiety and Benzodiazepine Abuse in Mice and Humans
}

\author{
Sonja M Wojcik,,$^{1^{*}}$ Martesa Tantra, ${ }^{2,3^{*}}$ Beata Stepniak, ${ }^{2^{*}}$ Kwun-nok M Man, ${ }^{1,3}$ Katja Müller-Ribbe, ${ }^{2}$ \\ Martin Begemann, ${ }^{2}$ Anes Ju, ${ }^{2}$ Sergi Papiol, ${ }^{2,3}$ Anja Ronnenberg, ${ }^{2}$ Artem Gurvich, ${ }^{2}$ Yong Shin, ${ }^{1,4}$ \\ Iris Augustin, ${ }^{1,5}$ Nils Brose, ${ }^{1,3}$ and Hannelore Ehrenreich ${ }^{2,3}$
}

${ }^{1}$ Max Planck Institute of Experimental Medicine, Department of Molecular Neurobiology, Göttingen, Germany; ${ }^{2}$ Max Planck Institute of Experimental Medicine, Clinical Neuroscience, Göttingen, Germany; ${ }^{3}$ DFG Center for Nanoscale Microscopy and Molecular Physiology of the Brain, Göttingen, Germany; ${ }^{4}$ present address: BioElectronics, Institute of Microelectronics, Agency for Science, Technology and Research (A*STAR), Singapore, Singapore; and ${ }^{5}$ present address: German Cancer Research Center, Department Signaling and Functional Genomics, Heidelberg, Germany

\begin{abstract}
Anxiety disorders and substance abuse, including benzodiazepine use disorder, frequently occur together. Unfortunately, treatment of anxiety disorders still includes benzodiazepines, and patients with an existing comorbid benzodiazepine use disorder or a genetic susceptibility for benzodiazepine use disorder may be at risk of adverse treatment outcomes. The identification of genetic predictors for anxiety disorders, and especially for benzodiazepine use disorder, could aid the selection of the best treatment option and improve clinical outcomes. The brain-specific angiogenesis inhibitor l-associated protein 3 (Baiap3) is a member of the mammalian uncoordinated 13 (Munc 13) protein family of synaptic regulators of neurotransmitter exocytosis, with a striking expression pattern in amygdalae, hypothalamus and periaqueductal gray. Deletion of Baiap3 in mice leads to enhanced seizure propensity and increased anxiety, with the latter being more pronounced in female than in male animals. We hypothesized that genetic variation in human BAIAP3 may also be associated with anxiety. By using a phenotype-based genetic association study, we identified two human BAIAP3 single-nucleotide polymorphism risk genotypes (AA for rs2235632, TT for rs 1132358 ) that show a significant association with anxiety in women and, surprisingly, with benzodiazepine abuse in men. Returning to mice, we found that male, but not female, Baiap3 knockout (KO) mice develop tolerance to diazepam more quickly than control animals. Analysis of cultured Baiap3 KO hypothalamus slices revealed an increase in basal network activity and an altered response to diazepam withdrawal. Thus, Baiap3/BAIAP3 is gender specifically associated with anxiety and benzodiazepine use disorder, and the analysis of Baiap3/BAIAP3-related functions may help elucidate mechanisms underlying the development of both disorders.
\end{abstract}

Online address: http://www.molmed.org

doi: $10.2119 / \mathrm{molmed} .2013 .00033$

\section{INTRODUCTION}

Anxiety disorders have high lifetime prevalence rates (1) and exhibit a remarkable comorbidity with substance use disorders (2-4). This association worsens treatment outcomes for both conditions (5) and represents a significant burden on individuals and society. Both anxiety disorders and substance use disorders are complex disorders that arise from a combination of genetic influence and environmental factors. To

*SMW, MT, and BS contributed equally to this study.

Address correspondence to Sonja M Wojcik, Max Planck Institute of Experimental Medicine, Department of Molecular Neurobiology, Hermann-Rein-Str. 3, D-37075 Göttingen, Germany. Phone: +49-551-3899-722; Fax: +49-551-3899-715; E-mail: wojcik@em.mpg.de. Submitted April 12, 2013; Accepted for publication May 14, 2013; Epub (www.molmed.org) ahead of print May 14, 2013.

The Feinstein Institute for Medical Research ShoreLIJ

improve upon established treatment options, which include pharmacological as well as cognitive-behavioral therapies $(6,7)$, a more detailed picture of the etiology of these disorders is instrumental. Estimates of heritability from twin and family studies are in the range of $20-40 \%$ across the different anxiety disorders $(8,9)$ and in the range of $40-70 \%$ for the major substance use disorders (10). Recent studies point to the involvement of a large number of genes with relatively small effect sizes for both anxiety disorder $(11,12)$ and substance use disorder (13-15). Although the interaction between anxiety disorders and substance use disorders is likely bidirec- 
tional and varies by the type of anxiety (16), genetically determined anxiousness personality traits may make the development of an addiction more likely (2,17-19). The recommended first-line pharmacological treatments of anxiety disorders are selective serotonin or serotonin/norepinephrine reuptake inhibitors and the calcium channel modulator pregabaline (6). However, primary care physicians often still prescribe benzodiazepines, which rank among the most frequently abused prescription medications (National Institute on Drug Abuse [http:/ / www.nida.nih.gov]), to patients suffering from anxiety disorders (20). Identifying genetic risk markers would advance our understanding of the biology of anxiety and benzodiazepine abuse and would be a valuable step in improving treatment options for these complex diseases.

In addition to human family, twin and genome-wide association studies, animal models are used to study the genetic basis and neural circuitries of anxiety and addiction. For both animals and humans, anxiety is an adaptive defensive response to threatening stimuli necessary for the survival of the species, whereas anxiety disorders are an extreme and maladaptive manifestation of normal anxiety (21). Somatic anxiety symptoms are mediated by the release of specific neurotransmitters and neuropeptides. The selection of candidate genes that are being investigated in animal studies is still largely driven by hypotheses of the neural circuitries and neurotransmitter systems thought to be involved in mediating fear and anxiety (22). By using a candidate gene approach, we investigate the involvement of the brain-specific angiogenesis inhibitor I-associated protein 3 (Baiap3), which is highly expressed in brain regions involved in processing fear, such as the amygdalae, hypothalamus and periaqueductal gray, in behavioral phenotypes relevant for human psychiatric disorders.

Baiap3 is a member of the mammalian uncoordinated 13 (Munc13) family of synaptic regulators of neurotransmitter exocytosis (23-25). Baiap3 has a unique and striking expression pattern (Allen Brain Atlas [http://mouse.brainmap.org]) in brain regions such as the central, medial and basomedial amygdaloid nuclei; the hypothalamus; and the periaqueductal gray. These areas are involved in regulating autonomic functions and are also critical in processing fearful stimuli and mediating anxiety-related behaviors $(26,27)$. The cellular function of Baiap3 is currently unknown; however, all other Munc13 members are regulators of vesicle exocytosis in various cell types (28). In the brain, Munc13-1 and Munc13-2 are essential for membrane fusion of synaptic vesicles containing classical neurotransmitters, such as glutamate or $\gamma$-aminobutyric acid (GABA) (25). Munc13-4, a non-neuronal Munc13 isoform most closely related to Baiap3 at the sequence level, is involved in exocytosis in cells of the hematopoietic system $(29,30)$.

To explore the function of Baiap3, we combined the behavioral analysis of Baiap3 knockout (KO) mice with a phenotype-based genetic association study (PGAS) of the human BAIAP3 gene by using the Göttingen Research Association for Schizophrenia (GRAS) database $(31,32)$. Using this two-pronged approach, we identify Baiap3/BAIAP3 as the first genetic risk marker for anxiety and benzodiazepine abuse in both mice and humans.

\section{MATERIALS AND METHODS}

\section{Animals}

Animal maintenance. All experiments were approved by the local Animal Care and Use Committee of Lower Saxony, Oldenburg, Germany. The first three coding exons of the murine Baiap3 gene were preplaced with a neomycin resistance cassette through homologous recombination in embryonic stem cells (129/Ola) (Supplementary Figure S1A). Baiap3 mutant mice of mixed 129/Ola;C57BL/6N background were backcrossed for seven more generations to C57BL/6N; all experiments were done with $\mathrm{WT}$ and $\mathrm{KO}$ littermates of the resulting generation 8 . After weaning, mice were group-housed in standard plastic cages $(n=5$ per cage) and maintained in a temperaturecontrolled environment $\left(21 \pm 2^{\circ} \mathrm{C}\right)$ on a 12-h light-dark cycle with food and water ad libitum, unless stated otherwise.

Drugs used in animal experiments. Two classical benzodiazepines, positive allosteric modulators of GABA type A receptors $\left(G_{A B A} R\right)$ were used: (i) the long-acting benzodiazepine diazepam (ratiopharm GmbH, Ulm, Germany) was suspended in saline containing polysorbate 80 for intraperitoneal (IP) injection, and (ii) the short-acting benzodiazepine midazolam (ratiopharm) was added to $2 \%$ sucrose solution for oral administration. Antagonists used were as follows: (i) flumazenil (Sigma-Aldrich Chemie, Munich, Germany), routinely applied in the clinic to counteract benzodiazepine overdoses, was dissolved in saline containing polysorbate 80 and $\mathrm{HCl}$; and (ii) pentylenetetrazole (PTZ) (Sigma-Aldrich Chemie), a noncompetitive GABA antagonist with epileptogenic properties, was dissolved in saline for IP injection.

Phenotypical characterization of Baiap3 KO mice. Behavioral characterization of naive Baiap3 $\mathrm{KO}$ mice and their WT littermates of both sexes began at the age of $8 \mathrm{wks}$ and was performed in the following order: elevated plus-maze, open field, light-dark box, hole board, rotarod and exposure to a fear-conditioning chamber to assess novelty-induced freezing behavior. Mouse numbers of all individual experiments are given in the figure legends.

Elevated plus-maze. The mouse was placed in the central platform, facing an open arm of the plus-maze. Behavior was recorded over $5 \mathrm{~min}$ by an overhead video camera. A personal computer equipped with Viewer software (Biobserve, Bonn, Germany) was used to calculate the time each animal spent in open versus closed arms. The proportion of time spent in open arms (natural aversion) was used as a fear equivalent.

Open field. Spontaneous activity in open field was tested in a gray Perspex 
arena (120 cm in diameter, $25 \mathrm{~cm}$ high), virtually divided into three zones: central, intermediate and peripheral. The mouse was placed in the center, and the test was started when the mouse reached the wall. Over $7 \mathrm{~min}$, the mouse was allowed to freely explore the open field. Behavior was recorded by a personal computer-linked overhead video camera and calculated using Viewer software. Readouts were as follows: velocity, distance traveled, time spent in each zone and initial latency to reach the wall.

Hole board. The hole board apparatus (TSE Systems GmbH, Bad Homburg, Germany) for measuring exploratory activity consisted of a $50 \mathrm{~cm} \times 50 \mathrm{~cm} \times$ $35 \mathrm{~cm}$ transparent Perspex chamber with a nontransparent floor raised above the bottom of the chamber. The floor had 16 equally spaced holes, $2.4 \mathrm{~cm}$ in diameter, fitted with a light barrier sensor $(8 \mathrm{~mm}$ below floor). Mice were allowed to explore the chamber for $5 \mathrm{~min}$, and the number of explored holes (head dips) was recorded.

Rotarod. This test for motor function, balance and coordination consists of a rotating drum (Ugo Basile, Comerio, Varese, Italy), accelerated from 4 to 40 revolutions per minute over $5 \mathrm{~min}$. Each mouse was placed individually on a drum and the latency of falling from the drum was recorded using a stopwatch. To assess motor learning, the test was repeated $24 \mathrm{~h}$ later.

Novelty-induced fear response. To assess novelty-induced fear response (indicated by freezing behavior), a chamber designed for training and testing of context fear conditioning was used. Mice were placed inside the chamber and allowed to explore the chamber freely for 2 min, during which time no additional stimulus was presented (equivalent to the assessment of baseline freezing of the fear-conditioning paradigm). Duration of freezing behavior, defined as the absolute lack of movement (excluding respiratory movements), was recorded by a video camera and a personal computer equipped with Video Freeze software (MED Associates, St. Albans, VT, USA).
Pentylenetetrazole-induced seizures. Seizure activity was induced in wakeful mice by using a single IP injection of PTZ (50 mg/kg body weight) (33). After injection of the compound, the mouse was placed in a small, clear home cage and closely observed for $30 \mathrm{~min}$. Latencies to focal (partial clonic), generalized (generalized clonic) and maximal (tonic-clonic) behavioral seizures were recorded. Furthermore, four phases in the continuum of behavioral response to IP PTZ injection were defined as follows: (i) hypoactivity (progressive decrease in motor activity until the animal came to rest in a crouched or prone position with the abdomen in full contact with the cage bottom); (ii) partial clonus (clonus seizure activity affecting face, head and/or forelimb or forelimbs); (iii) generalized clonus (sudden loss of upright posture, whole body clonus involving all four limbs and tail, rearing and autonomic signs); and (iv) tonic-clonic (maximal) seizure (generalized seizure characterized by tonic hindlimb extension-also associated with death). Finally, latencies to partial clonus (PC), generalized clonus (GC) and tonic-clonic (TC) seizures were summed to assign a seizure score to each mouse, used as a quantitative trait measure for mapping according to the following equation: seizure score $=[(0.2) \times$ $(1 /$ PC latency $)+(0.3) \times(1 /$ GC latency $)+$ $(0.5) \times(1 /$ TC latency $)] \times 1,000$. The

weighting factors $(0.2,0.3$ and 0.5$)$ in the equation were included as a means of incorporating a measure of the progressive nature of the PTZ-induced seizure phenotype into the severity rating because generalized clonus is regarded as a more significant event than partial clonus and tonic hind limb extension as the most severe component of the phenotype. Therefore, the seizure score reflects the degree of progression of the seizure phenotype in each mouse (33).

Diazepam dependence, tolerance and withdrawal. The mice received injections of diazepam ( $5 \mathrm{mg} / \mathrm{kg}$ body weight IP) for 10 consecutive days. Rotarod test was performed $30 \mathrm{~min}$ after each diazepam injection for $7 \mathrm{~d}$, with baseline rotarod training performed for $2 \mathrm{~d}$ before starting injections. On d 11, diazepam withdrawal was induced by flumazenil (15 mg/kg body weight IP), followed by injection of PTZ (50 mg/ $\mathrm{kg}$ body weight IP) to induce withdrawal-related seizures. Seizure induction by PTZ (50 mg/kg body weight IP) was also performed on drug-naive mice.

Midazolam oral self-administration and behavior testing in the addicted state. To induce benzodiazepine dependence as a prerequisite for oral selfadministration (document of addiction), group-housed mice received midazolam (ratiopharm) in 2\% sucrose (to reduce the bitter taste), instead of drinking water. Midazolam concentration was increased weekly, starting from $0.005 \mathrm{mg} / \mathrm{mL}$ until the maximum concentration of $0.05 \mathrm{mg} / \mathrm{mL}$ was reached after $10 \mathrm{wks}$. A respective control group received $2 \%$ sucrose only. One set of midazolam mice was then exposed to a midazolam preference test. For this purpose, mice were first switched to single housing with a continued supply of midazolam $(0.05 \mathrm{mg} / \mathrm{mL})$ for $2 \mathrm{wks}$. For the preference test, every mouse had a choice of two bottles containing either midazolam $(0.05 \mathrm{mg} / \mathrm{mL})$ in $2 \%$ sucrose or $2 \% \mathrm{su}-$ crose alone for another $2 \mathrm{wks}$. The relative consumption of midazolam solution was calculated. The other set of mice (midazolam and control mice) stayed group-housed and underwent automated home cage observation using the LABORAS $^{\mathrm{TM}}$ system (Metris, Hoofddorp, Netherlands). LABORAS is a fully automated system for continuous behavior recognition and tracking in small rodents. For habituation before testing, mice were temporarily put in single cages similar to the LABORAS cage in the testing room for 2 consecutive nights (1700 to 0900). On the day of testing, Makrolon type 3 cages $\left(840 \mathrm{~cm}^{2}\right)$, filled with a 2-cm layer of bedding used during the habituation phase, were placed on each triangular sensor platform $(95 \mathrm{~cm} \times 75 \mathrm{~cm} \times 75 \mathrm{~cm})$. Food and sucrose solution with midazolam (addicted group) or 2\% sucrose (control group) were provided ad libitum. 
Before each session, LABORAS was calibrated by using the calibration procedure and reference weights supplied by Metris. Movements during nighttime (1800 to 0900) were recorded and distinguished as separate behavioral patterns by the LABORAS software. Locomotion duration and scratching frequency during the dark phase (2000 to 0800) was analyzed.

Statistical analysis. Behavioral data were analyzed separately for males and females by the Mann-Whitney $U$ test and two-way analysis of variance (ANOVA), including post hoc Bonferroni testing, where applicable, using Prism4 (GraphPad Software, San Diego, CA, USA). Significance level was set to $p<0.05$. All data are presented as mean \pm standard error of the mean (SEM).

\section{Human Sample}

Schizophrenic patient sample. The schizophrenic patient sample $(n=1,086)$ was recruited across 23 sites throughout Germany in the cross-sectional GRAS study and most comprehensively phenotyped $(31,32)$. The study was approved by the Ethics Committee of the GeorgAugust-University (Göttingen, Germany) and the review boards of participating centers and complies with the Declaration of Helsinki. Patients fulfilling Diagnostic and Statistical Manual of Mental Disorders: DSM-IV-TR, 4th edition, text revision (34) criteria for schizophrenia or schizoaffective disorder were included in the analyses regardless of their disease stage (acute, chronic, residual or remitted). Almost all patients were of European Caucasian descent (Caucasian 94.7\%; other ethnicities $1.9 \%$; unknown $3.4 \%$ ).

Healthy control sample. Voluntary blood donors $(n=1,142)$ recruited following the national guidelines for blood donation were included for case control analysis $(31,32)$. Also the majority of control subjects are of European Caucasian ethnicity (Caucasian 97.8\%; other ethnicities $2 \%$; unknown $0.2 \%$ ).

Sociodemographic and clinical variables. Sociodemographic data (age, years of education, level of unemployment), information on substance use disorder (summarizing abuse and dependence based on the DSM-IV-TR criteria for alcohol and cannabis) and clinical variables describing disease severity were used to characterize the sample. Clinical variables included Positive and Negative Syndrome Scale (PANSS) positive scale as a measure of positive symptom severity (35) as well as chlorpromazine equivalents to estimate the relative dose of antipsychotic medication. The Global Assessment of Functioning (GAF) scale (DSM-IV-TR) was used as a measure of impairment of psychological, social and occupational functioning.

Target variables. The dichotomous DSM-IV-TR benzodiazepine use disorder diagnosis (summarizing abuse and dependence) and the quantitative anxiety composite score were our target variables. The anxiety composite score is based on the aggregation of four anxietyrelated variables: (i) Brief Symptom Inventory (BSI) subscale anxiousness; (ii) State-Trait Anxiety Inventory (STAI) subscale trait anxiety; (iii) STAI subscale state anxiety; and (iv) anxiety item of the PANSS general psychopathology subscale (Supplementary Figure S2).

DNA extraction and normalization. Genomic DNA was purified from whole blood by using JETQUICK Blood and Cell Culture DNA Spin Kit (Genomed, Loehne, Germany) according to the manufacturer's protocol. DNA aliquots were stored at $-80^{\circ} \mathrm{C}$. For further analyses, DNA was normalized to $50 \mathrm{ng} / \mu \mathrm{L}$ with an automated robotic platform (Microlab Star, Hamilton, Bonaduz, Switzerland). Each sample was analyzed with a $0.8 \%$ agarose gel for quality control.

Genotyping. The three selected SNPs (rs11648169, rs2235632, rs1132358) of BAIAP3 were analyzed by using Simple Probes (TIB Molbiol, Berlin, Germany) and genotyped using the LightCycler ${ }^{\circledR}$ 480 Genotyping Software implemented in the LightCycler 480 system (Roche, Mannheim, Germany). The reaction mixture $(10 \mu \mathrm{L})$ was prepared with $20 \mathrm{ng}$ DNA in 384-well plates following the standard protocol (Roche). In each run, eight positive controls (hgDNA, Bioline, Luckenwalde, Germany) and negative water blanks were included for quality and internal control purposes. Of the GRAS patients, a total of $n=1,082$ (99.63\%) were successfully genotyped for BAIAP3 SNP1 (C/G) rs11648169, $\mathrm{n}=$ 1,086 (100\%) for BAIAP3 SNP2 (G/A) rs2235632 and $\mathrm{n}=1,069$ (98.43\%) for BAIAP3 SNP3 (C/T) rs1132358 and included in the analyses. Of the healthy control subjects, all $n=1,142$ were successfully genotyped for SNP1, SNP2 and SNP3 of the BAIAP3 gene.

Statistical analyses. For all analyses, statistical significance was set to 0.05 . Statistical analyses of human data were performed by using SPSS for Windows, version 17.0. Group differences in categorical and continuous variables were assessed using $\chi^{2}$ or Mann-Whitney $U$ tests; in cases of normal distribution of the continuous variable, $t$ tests were performed. Anxiety score composition was done using $z$-standardized mean subscale scores (BSI anxiousness, STAI trait anxiety, STAI state anxiety) or, in the case of PANSS anxiety, a z-standardized single item, organized such that higher values represent higher symptom severity. Intercorrelations and internal consistency of the anxiety composite score was calculated by using Pearson correlation coefficient and Cronbach $\alpha$ (36). In the GRAS sample, the following items or scales were incomplete: BSI anxiousness $7.5 \%$ missing, STAI trait anxiety $20.2 \%$, STAI state anxiety $21.6 \%$ and PANSS anxiety $3.2 \%$. If all four anxiety variables were available, the mean was calculated for each respective subject as an individual anxiety composite score. In the case of missing data, a linear regression-based multiple imputation model (10 iterations) of missing data was applied, if at least three out of the four variables per subject were available. For the 190 individuals with imputed values, the final anxiety composite score represents the mean of 10 imputed values for the missing item, increasing the availability of the anxiety score from $n=771$ to $n=961$ schizo- 
phrenic subjects (37). Analysis of covariance (adjusted for age, PANSS positive subscale score and chlorpromazine equivalents) was used to analyze the effect of SNP genotypes on the standardized anxiety composite score. For the phenotype-genotype association analyses (including peripheral blood mononuclear cells [PBMCs]; see below) of the BAIAP3 SNP rs2235632, G carriers (GG and AG) were aggregated and contrasted with individuals homozygous for the A allele, and in the case of SNP rs1132358, C carriers (CC and TC) were aggregated and contrasted with TT individuals. SNP rs11648169 was excluded from further analyses, since it yielded no statistically significant effects.

\section{In Vitro Analyses}

Immunofluorescence analysis. Brains were perfusion-fixed, and organotypic hypothalamus slices were immersion fixed in $4 \%$ paraformaldehyde in phosphate buffer ( $\mathrm{pH}$ 7.4). Brains were postfixed for $1 \mathrm{~h}$, cryoprotected with 30\% sucrose and frozen. For immunofluorescence analysis, free-floating brain sections of $40 \mu \mathrm{m}$ thickness or organotypic sections of $300 \mu \mathrm{m}$ thickness were incubated in primary antibodies for $72 \mathrm{~h}$ followed by incubation with IgG-coupled Alexa Fluor 488, Alexa Fluor 555 and Alexa Flour 633 dyes (Invitrogen [Life Technologies, Darmstadt, Germany]) for $24 \mathrm{~h}$. Rabbit and guinea pig antibodies to Baiap3 were raised to a purified fragment (amino acids 617-973) containing the munc homology domain (MHD)-1 and MHD-2 of mouse Baiap3. Commercial primary antibodies used were rabbit and guinea pig anti-vesicular glutamate transporter 1 (VGLUT1), rabbit and guinea pig anti-VGLUT2, rabbit and guinea pig anti-vesicular inhibitory amino acid transporter (Viaat), mouse anti-Gephyrin (mAB7a) (all from Synaptic Systems, Göttingen, Germany), and mouse anti-postsynaptic density protein 95 (PSD-95) (clone K28/48, NeuroMab). False color images of brain sections and organotypic slices were obtained with a fluorescence stereomicroscope (Leica
FluoCombi III ${ }^{\mathrm{TM}}$ ) and an ApoTome ${ }^{\mathrm{TM}}$ fluorescence microscope (Axio Imager Z1; Zeiss), respectively.

Hypothalamus slice culture. Organotypic hypothalamus slices of 300- $\mu \mathrm{m}$ thickness from postnatal d 5 (P5) and P6 mice were prepared in Hanks balanced salt solution (24020-091; Invitrogen [Life Technologies]) with $20 \%$ glucose and $1 \mathrm{mmol} / \mathrm{L}$ kynurenic acid (SigmaAldrich, Germany) (pH 7.4), by using a Mcllwain Tissue Chopper. Slices were cultured in six-well plates on confetti cut from 0.45- $\mu$ m filters (FHLC04700; EMD Millipore [Millipore Ireland B.V., Tullagreen, Carrigtwohill County Cork, Ireland]) that were placed in 0.4- $\mu \mathrm{m}$ Millicell cell culture inserts (PICM03050; Millipore) for $5 \mathrm{~d}$ using a mixture of $41 \%$ Earle basal medium Eagle (BME) (F 0225; Biochrom, Berlin, Germany), with 25\% Earle balanced salt solution $(1.8 \mathrm{mmol} / \mathrm{L}$ $\mathrm{CaCl}_{2}, 1 \mathrm{mmol} / \mathrm{L} \mathrm{NaH}_{2} \mathrm{PO}_{4}, 0.8 \mathrm{mmol} / \mathrm{L}$ $\mathrm{MgSO}_{4}, 116 \mathrm{mmol} / \mathrm{L} \mathrm{NaCl}, 26.2 \mathrm{mmol} / \mathrm{L}$ $\mathrm{NaHCO}_{3}, 5.4 \mathrm{mmol} / \mathrm{L} \mathrm{KCl}, 5 \mathrm{mmol} / \mathrm{L}$ glucose), 20\% heat-inactivated horse serum, $10 \% \mathrm{H}_{2} \mathrm{O}, 25 \mathrm{mmol} / \mathrm{L}$ 4-(2-hydroxyethyl)1-piperazineethanesulfonic acid (HEPES) (Biochrom), $28 \mathrm{mmol} / \mathrm{L}$ glucose, $1 \mathrm{mmol} / \mathrm{L}$ GlutaMAX $^{\mathrm{TM}}$ (35050; Invitrogen [Life Technologies]), $1 \mu \mathrm{g} / \mathrm{mL}$ insulin, $88 \mu \mathrm{g} / \mathrm{mL}$ ascorbic acid, $0.25 \%$ MEM Vitamine Solution (K0373; Biochrom) and 0.5\% MEM Amino Acids (K0363; Biochrom). On d 5 in vitro, the cultures were switched to a medium with identical components but containing 5\% horse serum, 55\% BME and $2 \mathrm{mmol} / \mathrm{L}$ GlutaMAX. At the same time, diazepam was added to the medium from a $60 \mathrm{mmol} / \mathrm{L}$ stock solution in dimethylsulfoxide (DMSO) for a final concentration of $10 \mu \mathrm{mol} / \mathrm{L}$. For control cultures, DMSO was added as a vehicle control at the same dilution of 1:6,000. The $\mathrm{CO}_{2}$ concentration was $5 \%$, and medium changes were done on the day after culture and every $48 \mathrm{~h}$ after that.

Electrophysiological analyses. Organotypic slices containing the ventromedial hypothalamus were transferred to the recording chamber between DIV10 and DIV17. Recordings were started after a 30-min recovery time, the extracellular recording solution contained $120 \mathrm{mmol} / \mathrm{L}$ $\mathrm{NaCl}, 26 \mathrm{mmol} / \mathrm{L} \mathrm{NaHCO}_{3}, 1 \mathrm{mmol} / \mathrm{L}$ $\mathrm{KH}_{2} \mathrm{PO}_{4}, 2 \mathrm{mmol} / \mathrm{L} \mathrm{KCl}, 20 \mathrm{mmol} / \mathrm{L} \mathrm{glu}-$ cose, $2 \mathrm{mmol} / \mathrm{L} \mathrm{MgCl}_{2}, 2 \mathrm{mmol} / \mathrm{L} \mathrm{CaCl}_{2}$ and $250 \mathrm{nmol} / \mathrm{L}$ flumazenil. Cells were whole-cell voltage clamped at -70 or $-20 \mathrm{mV}$ or recorded in current clamp mode with an EPC 10 USB Double (HEKA, Lambrecht/Pfalz, Germany) under control of the Patchmaster 2.52 program (HEKA). All analyses were performed by using the Mini Analysis Program (Synaptosoft, Decatur, GA, USA). Recordings of miniature inhibitory postsynaptic currents (mIPSCs) were performed in the presence of $1 \mu \mathrm{mol} / \mathrm{L}$ tetrodotoxin (Tocris [R\&D Systems, Wiesbaden-Nordenstadt, Germany]) and $10 \mu \mathrm{mol} / \mathrm{L} 2,3-$ dioxo-6-nitro-1,2,3,4tetrahydrobenzo[ $f]$ quinoxaline-7sulfonamide (NBQX) (Tocris [R\&D Systems]), with an intracellular solution containing $100 \mathrm{mmol} / \mathrm{L} \mathrm{KCl}, 50 \mathrm{mmol} / \mathrm{L}$ K-gluconate, $10 \mathrm{mmol} / \mathrm{L}$ HEPES, $0.1 \mathrm{mmol} / \mathrm{L}$ EGTA, $0.3 \mathrm{mmol} / \mathrm{L} \mathrm{GTP}$, $4 \mathrm{mmol} / \mathrm{L} \mathrm{ATP}$ and $0.2 \%$ biocytin. Action potentials and spontaneous inhibitory postsynaptic currents (IPSCs) were recorded with an intracellular solution containing $20 \mathrm{mmol} / \mathrm{L} \mathrm{KCl}, 130 \mathrm{mmol} / \mathrm{L} \mathrm{K}$-gluconate, $10 \mathrm{mmol} / \mathrm{L}$ HEPES, $0.1 \mathrm{mmol} / \mathrm{L}$ EGTA, $0.3 \mathrm{mmol} / \mathrm{L}$ GTP, $4 \mathrm{mmol} / \mathrm{L}$ ATP and $0.2 \%$ biocytin. Action potentials analyzed were from the first minute of a 2-min recording; membrane potentials were measured after setting the current injection to $0 \mathrm{pA}$ at the end of the recording. IPSCs were recorded for $5 \mathrm{~min}$ after switching the cell to a holding potential of $-20 \mathrm{mV}$ and waiting for $1 \mathrm{~min}$. Statistical analyses were performed using GraphPad Prism5.

Analysis of BAIAP3 mRNA levels in PBMCs. PBMCs from 121 patients were isolated by using the standard FicollPaque Plus isolation procedure (GE Healthcare, Munich, Germany). For RNA isolation, the miRNeasy Mini Kit (Qiagen, Hilden, Germany) was used. A total of $1 \mu \mathrm{g}$ RNA, a mixture of oligo dT, hexamer primers, dNTPS (10 mmol/L each) and SuperScriptIII (200 U; Invitrogen [Life Technologies]) were used for transcription into cDNA (20- $\mu \mathrm{L}$ reaction). The mixture 
was incubated for $10 \mathrm{~min}$ at $25^{\circ} \mathrm{C}$ and 45 min at $50^{\circ} \mathrm{C}$, followed by $45 \mathrm{~min}$ at $55^{\circ} \mathrm{C}$. For the quantitative reverse transcriptase polymerase chain reaction (qRT-PCR), a 1:10 dilution of the cDNA was used and three replicate experiments per sample were performed: $5 \mu$ L Power SYBR mix (Applied Biosystems) and 1 pmol of each primer were added. BAIAP3 qRTPCR primers used were as follows: $5^{\prime}-$

AGCTGGGCCCACCGCATCTCT-3' with 5'-CTCGGCAGGCACGGAAAAGTAG-3' and 5'-CTGACTTCAACAGCGACACC-3' with 5'-TGCTGTAGCCAAATTCGTTGT-3'. The following cycling profile was run on the LightCycler480 system (Roche): preheating at $95^{\circ} \mathrm{C}$ for $10 \mathrm{~min}$; 45 cycles of $95^{\circ} \mathrm{C}$ for $15 \mathrm{~s}, 60^{\circ} \mathrm{C}$ for $1 \mathrm{~min}$. Cycle threshold values of BAIAP3 were standardized to cycle threshold values of GAPDH.

All supplementary materials are available online at www.molmed.org.

\section{RESULTS}

\section{Generation of Baiap3 KO Mice}

Baiap3 shares the basic domain structure of other Munc13 isoforms, with two munc-homology domains flanked by two C2 domains but lacks the N-termini contained in Munc13-1, -2 and -3 (23). The murine Baiap3 gene contains 33 coding exons that span $8.7 \mathrm{~kb}$. We generated Baiap3 KO mice by homologous recombination in embryonic stem cells, replacing the first three coding exons with a neomycin selection cassette (Supplementary Figure S1A). Baiap3 KO mice are viable, fertile and indistinguishable from their wild-type (WT) littermates in the home cage. In WT brain, the expression pattern of Baiap3 protein analyzed by immunofluorescence staining largely matches the distribution of Baiap3 mRNA published in the Allen Brain Atlas. Baiap3 protein is prominently expressed throughout the hypothalamus and in the central, medial and basomedial amygdaloid nuclei, as well as in the paraventricular nucleus of the thalamus (Figure 1). Strong expression is further detected in the septum, bed nucleus of the stria terminalis, midbrain including the periaqueductal gray and inferior colliculus, and brain stem including the parabrachial nucleus and nucleus tractus solitarius (Figure 1). Baiap3 immunoreactivity appears punctate, but does not seem to localize to either glutamatergic or GABAergic pre- or postsynapses to a significant degree (Supplementary Figures S1C-G). Adult Baiap3 KO mice lack any detectable expression of Baiap3 protein by immunofluorescence and Western blot analysis (Figure 1B, Supplementary Figure S1B). Western blot analysis of brains taken from newborn Baiap3 $\mathrm{KO}$ animals revealed the presence of a weak band that most likely corresponds to Baiap3 protein expressed from a start codon present in coding exon 4; however, this putative truncated Baiap3 product is barely detectable by the age of $3 \mathrm{wks}$ and not present in adult animals (Supplementary Figure S1B).

\section{Novelty-Induced Anxiety in Baiap3 KO Mice}

The striking expression pattern of Baiap3 in the amygdala and other brain regions involved in processing fear piqued our interest, and we chose to assess whether the genetic deletion of Baiap3 led to any detectable behavioral alterations. We subjected Baiap3 KO mice and WT littermates of both sexes to a battery of standard behavioral tests (Figures 2A-L; Supplementary Figures S3A-J). In the open field, both male and female Baiap3 $\mathrm{KO}$ mice showed an increased latency to reach the wall upon release in the center zone (Figures 2A, B). Female but not male $\mathrm{KO}$ mice also made fewer visits to the center (Figures 2C, D) and spent significantly more time in the periphery (Figures 2E, F). When placed in a novel chamber (fear-conditioning box), both male and female $\mathrm{KO}$ mice showed an increased novelty-induced freezing response (Figures 2K, L). Taken together, these findings are indicative of a heightened novelty-induced anxiety level in Baiap3 $\mathrm{KO}$ animals, with a more pronounced effect noted in females. In contrast, classical tests, measuring anxi- ety in the context of an inherent conflict between a protected and a more anxiogenic area, that is, elevated plus-maze and light-dark box, did not reveal any genotype differences (Figures 2G-J). Furthermore, the distance traveled (motor activity) in open field and elevated plusmaze (Supplementary Figures S3A-D), exploratory behavior (hole board; Supplementary Figures S3E, F), motor learning and coordination (rotarod; Supplementary Figures S3G, H) and body weight (Supplementary Figures S3I, J) were not affected by genotype.

\section{BAIAP3 Is a Risk Marker for Anxiety in Women}

To explore the possibility of an association of genetic variability in the human $B A I A P 3$ gene with specific biological readouts, we made use of the GRAS database of schizophrenic patients $(31,32)$. Our hypotheses regarding Baiap3/BAIAP3 function were based on the anxiety phenotype observed in Baiap3 $\mathrm{KO}$ mice and on the prominent expression of Baiap3 in brain regions involved in processing fearful stimuli as well as in substance use disorders. We selected three single-nucleotide polymorphisms (SNPs) in the BAIAP3 gene: rs11648169 (C/G, intronic), rs2235632 (G/A, intronic) and rs1132358 (C/T, coding sequence, synonymous Asp1040Asp) (Supplementary Figure S4A) from public databases [http:/ / www.ncbi. nlm.nih.gov/projects/SNP/; http:/ / browser.1000genomes.org; http:/ / hapmap.ncbi.nlm.nih.gov/]. The selection of SNPs was based on (i) a high minor allele frequency (MAF $\geq 0.36$ ) distribution within the European Caucasian population (http:/ / www.ncbi.nlm.nih.gov/ $\mathrm{SNP} /$ ), to increase the power to detect genetic effects, and (ii) the potential for functional consequences. The last criterion could only partially be fulfilled; the exonic SNP rs1132358 (C/T, Asp1040Asp, synonymous) might potentially affect mRNA structure or stability. All SNPs fulfilled Hardy-Weinberg equilibrium criteria, both in cases and in controls $(p>$ 0.05). A construction of haplotype blocks of the three SNPs revealed a similarly 

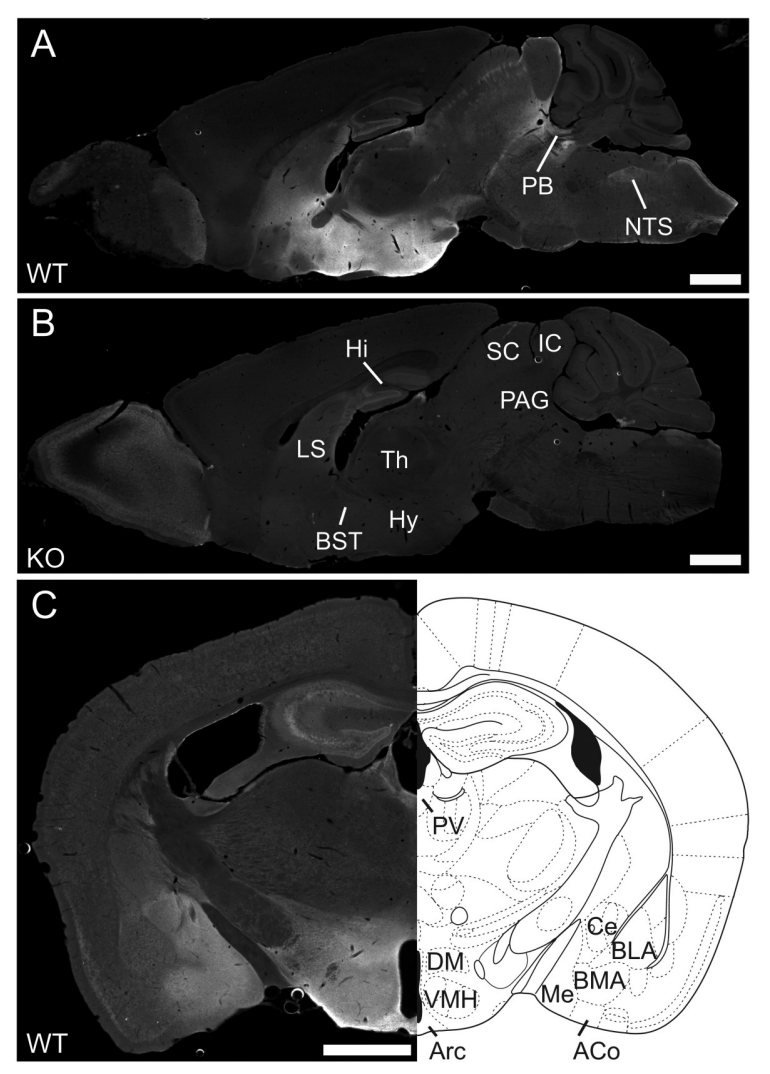

Figure 1. Immunofluorescence analysis of Baiap3 expression in mouse brain. (A) Sagittal brain section of adult Baiap3 WT mouse stained with rabbit anti-Baiap3 antibody. (B) Sagittal brain section of adult Baiap3 KO littermate showing the absence of Baiap3 immunoreactivity. Please note that the signal observed in the hippocampus of both WT and $\mathrm{KO}$ brain is a nonspecific background staining. (C) Coronal brain section of adult WT mouse stained for Baiap3 with a corresponding coronal diagram, adapted from the mouse Paxinos brain atlas (Bregma -1.46). PB, parabrachial nucleus; NTS, nucleus tractus solitarius; Hi, hippocampus; SC, superior colliculus; IC, inferior colliculus; PAG, periaqueductal gray; LS, lateral septum; Th, thalamus; Hy, hypothalamus; BST, bed nucleus of the stria terminalis; PV, paraventricular thalamic nucleus; DM, dorsomedial hypothalamic nucleus; $\mathrm{VMH}$, ventromedial hypothalamic nucleus; Arc, arcuate nucleus; Ce, central amygdaloid nucleus; BLA, basolateral amygdaloid nucleus, anterior part; BMA, basomedial amygdaloid nucleus, anterior part; ME, medial amygdaloid nucleus; ACo, anterior cortical amygdaloid nucleus. Scale bars equal $1 \mathrm{~mm}$.

high degree of linkage disequilibrium between them in the GRAS sample (Supplementary Figure S4B) and in healthy controls (Supplementary Figure S4C). Case control analysis of genotype frequencies of the three SNPs did not reveal any significant differences, indicating that the selected genetic variation in BAIAP3 is not associated with schizophrenia risk (Supplementary Figure S4D). We subsequently used the PGAS approach (32) to analyze the three SNPs for association with specific phenotypic readouts rele- vant for anxiety disorders and substance use disorders. For this step, an anxiety composite score was constructed using four anxiety-relevant variables (Supplementary Figure S2), which showed a significant association with only two of the three selected SNPs (as expected because of the high linkage disequilibrium between both markers and their similar MAFs) for women but not for men (Table 1). SNP rs11648169 was excluded from further analyses, since it yielded no statistically significant effects.

\section{BAIAP3 Is a Risk Gene for Benzodiazepine Abuse in Men}

Because anxiety disorders and substance use disorders often occur together, and BAIAP 3 is expressed in brain regions relevant for emotionality and drug dependence, we also screened for a possible association between genetic variation in $B A I A P 3$ and substance use disorder. The same risk genotypes (AA for rs2235632, TT for rs1132358) that were associated with anxiety in women showed a statistically significant association with benzodiazepine use disorder in men (Table 1). Even though there was a similar tendency for women (benzodiazepine use disorder associated with $7.0 \% / 7.7 \%$ in $\mathrm{AA} / \mathrm{TT}$ genotypes versus $4.7 \% / 4.6 \%$ in $\mathrm{G} / \mathrm{C}$ carrier status), it did not reach statistical significance, perhaps because of the lower numbers of women than men in the GRAS sample. The genotype frequencies of rs2235632 and rs1132358 did not differ between men and women in the GRAS sample (rs2235632, GG/AG/AA: men 25.1\%/49.1\%/25.8\%; women $28.3 \% / 46.3 \% / 25.5 \%$; rs 1132358 , CC/TC/TT: men 26.1\%/49.4\%/24.5\%; women $29.8 \% / 46.9 \% / 23.3 \%$ ), and neither of these two SNPs was associated with disease-related or sociodemographic control variables (Table 1 ).

For the purpose of an association analysis of the relevant BAIAP3 genotypes (GG/AG/AA in rs2235632 and CC/TC/TT in rs1132358) with benzodiazepine use disorder, the GRAS sample delivers an ideal, nearly experimental setting. The distribution of these genotypes among benzodiazepine users versus nonusers is highly comparable, allowing the identification of risk genotypes leading to benzodiazepine use disorder (Supplementary Table S1). Most importantly, the benzodiazepine dose was equal across all genotypes (Supplementary Table S1). Hence, the BAIAP3 risk genotypes (AA for rs2235632, TT for rs1132358) appear to confer a specific genetic risk of developing benzodiazepine use disorder given equal dose and likelihood of exposure. Interestingly, neither alcohol nor cannabis abuse were found to 


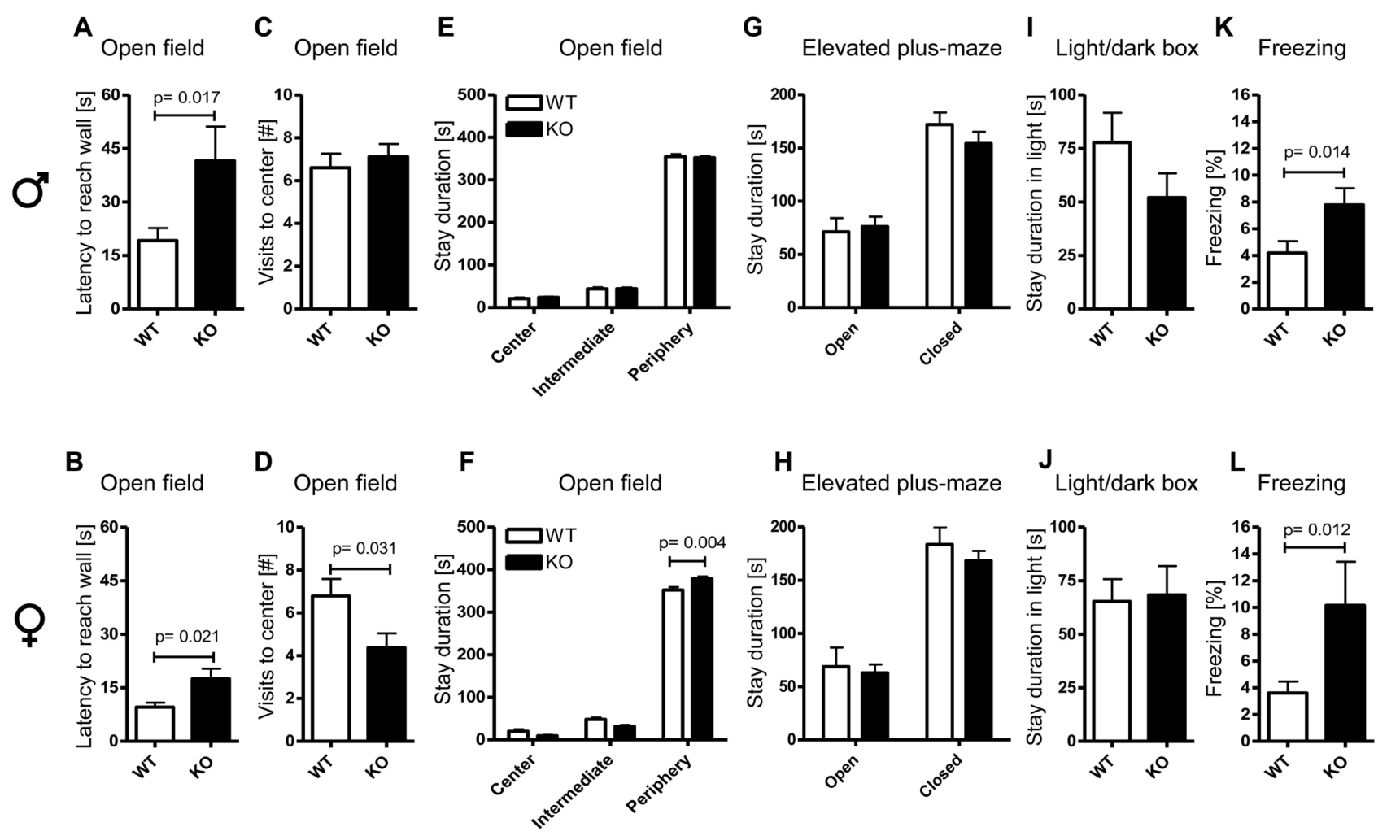

Figure 2. Anxiety phenotype in Baiap3 $\mathrm{KO}$ mice. (A-F) Open field parameters. (A, B) The latency to reach the wall of the open field was significantly increased in Baiap3 $\mathrm{KO}$ mice of both sexes, whereas visits to the center (C, D) and stay in the periphery (E, F) revealed anxiety-like behavior only in females. Elevated plus-maze $(G, H)$ and light-dark box $(\mathrm{I}, \mathrm{J})$ revealed no genotype-dependent differences in either sex. $(K, L)$ As readout of unspecific novelty-related anxiety, a higher freezing response was found in male as well as female Baiap3 KO mice. Numbers tested: males, $\mathrm{WT}=16-25, \mathrm{KO}=16-25$; females, WT = 18-23, $\mathrm{KO}=10-28$. Mann-Whitney U test (A-D, I-L) and two-way ANOVA $(E-H)$, including Bonferroni testing, were applied. Means \pm SEM are presented.

be associated with the two SNPs, pointing to a specific benzodiazepine link with the selected BAIAP3 genotypes (Table 1).

To determine whether the identified risk genotypes are associated with altered expression of BAIAP3, we analyzed the mRNA levels of BAIAP3 in PBMCs obtained from 121 subjects by qRT-PCR. We found a statistically significant association of the BAIAP3 risk genotypes (AA for rs2235632, TT for rs1132358) with lower BAIAP3 mRNA levels in PBMCs of male individuals, which is at least partially comparable to a gene dose reduction or KO situation. This result is not found in women, possibly because of the lower numbers available for analysis (Supplementary Figure S5). However, these findings could also support the interpretation that the effects of BAIAP3 risk alleles are gender specific.

\section{Male Baiap3 KO Mice Show Faster Development of Tolerance to Benzodiazepines}

On the basis of the identification of human BAIAP3 risk genotypes for benzodiazepine abuse in male patients, we tested Baiap3 KO and WT littermates of both sexes in experimental paradigms of chronic benzodiazepine administration to assess the development of tolerance, dependence and withdrawal (Figure 3A). The baseline performance of each mouse in the rotarod test was established on two consecutive days of rotarod training. No significant genotype-dependent differences were detected in baseline performance (Supplementary Figures S3G, $\mathrm{H})$. Benzodiazepine dependence in Baiap3 KO and WT mice of both sexes was then induced with daily diazepam injections (5 mg/kg IP) for 10 consecu- tive days. To monitor the development of tolerance to diazepam, motor performance on rotarod at $30 \mathrm{~min}$ after each injection was evaluated over the first $7 \mathrm{~d}$ of diazepam treatment. Rapid development of tolerance to daily diazepam injections was apparent in both sexes and genotypes by an increase of the latency of falling from rotarod over the course of $7 \mathrm{~d}$ (Figures 3B, C). Here, male Baiap3 KO mice performed significantly better than their WT littermates (Figure 3B), whereas no such difference was detected for females (Figure 3C). Thus, male Baiap3 $\mathrm{KO}$ mice show faster development of tolerance to diazepam.

\section{Baiap3 KO Mice Have an Increased Seizure Propensity}

To evaluate whether Baiap3 genotype would affect the propensity for di- 
Table 1. Phenotype comparison of GRAS patients sorted by BAIAP3 genotypes.

\begin{tabular}{|c|c|c|c|c|c|c|}
\hline \multirow[b]{2}{*}{ GRAS sample } & \multicolumn{3}{|c|}{ BAIAP3 rs2235632 } & \multicolumn{3}{|c|}{ BAIAP3 rs 1132358} \\
\hline & $\begin{array}{l}\text { G carriers } \\
\text { (GG/AG) }\end{array}$ & AA & $\begin{array}{c}p \\
\left(F / T / Z / \chi^{2} \text { value }\right)^{a}\end{array}$ & $\begin{array}{l}\text { C carriers } \\
\text { (CC/TC) }\end{array}$ & TT & $\begin{array}{c}p \\
\left(F / T / Z / \chi^{2} \text { value }\right)^{a}\end{array}$ \\
\hline Males & $n=471-538^{b}$ & $n=155-187^{b}$ & & $n=474-538^{b}$ & $n=152-175^{b}$ & \\
\hline \multicolumn{7}{|l|}{ Target variables } \\
\hline Benzo use disorder ( $(\%)$ ) & $11(2.3)$ & $9(5.4)$ & $0.047\left(\chi^{2}=3.93\right)$ & $11(2.3)$ & $9(5.7)$ & $0.034\left(\chi^{2}=4.47\right)$ \\
\hline Anxiety composite score $(\text { mean } \pm S D)^{c, d}$ & $-0.06 \pm 0.74$ & $-0.07 \pm 0.70$ & $0.499(F=0.46)$ & $-0.07 \pm 0.73$ & $-0.05 \pm 0.72$ & $0.651(F=0.21)$ \\
\hline \multicolumn{7}{|l|}{ Sociodemographic } \\
\hline Age (at examination) (years) (mean \pm SD (range)) & $37.33 \pm 12.01(18-78)$ & $36.17 \pm 11.91(17-75)$ & $0.225(Z=-1.21)$ & $37.28 \pm 11.96(18-78)$ & $36.34 \pm 12.06(17-75)$ & $0.309(Z=-1.02)$ \\
\hline Education (years) $\left(\right.$ mean $\pm S D(\text { range) })^{e}$ & $14.17 \pm 3.48(8-28)$ & $14.42 \pm 3.70(8-27)$ & $0.680(Z=-0.41)$ & $14.21 \pm 3.48(8-28)$ & $14.32 \pm 3.76(8-27)$ & $0.853(Z=-0.19)$ \\
\hline Unemployment (n (\%)) & $217(44.7)$ & $76(44.7)$ & $0.990\left(\chi^{2}=0.00\right)$ & $214(44.2)$ & $75(46.6)$ & $0.601\left(\chi^{2}=0.27\right)$ \\
\hline \multicolumn{7}{|l|}{ Substance use } \\
\hline Alcohol use disorder according to DSM-IV-TR (n (\%)) & $221(42.3)$ & $84(45.7)$ & $0.435\left(\chi^{2}=0.61\right)$ & $217(41.6)$ & $82(47.4)$ & $0.180\left(\chi^{2}=1.80\right)$ \\
\hline Cannabis use disorder according to DSM-IV-TR (n (\%)) & $218(41.8)$ & $83(45.1)$ & $0.430\left(\chi^{2}=0.62\right)$ & $224(42.9)$ & $75(43.4)$ & $0.919\left(\chi^{2}=0.01\right)$ \\
\hline \multicolumn{7}{|l|}{ Clinical } \\
\hline PANSS positive score (mean \pm SD (range)) & $13.55 \pm 6.04(7-36)$ & $14.02 \pm 6.41(7-38)$ & $0.427(Z=-0.79)$ & $13.53 \pm 6.03(7-36)$ & $14.23 \pm 6.50(7-38)$ & $0.249(Z=-1.15)$ \\
\hline Chlorpromazine equivalents (mean \pm SD (range)) & $\begin{array}{c}707.90 \pm 694.69 \\
(0-6,324.29)\end{array}$ & $\begin{array}{c}689.45 \pm 568.91 \\
(0-3,238.00)\end{array}$ & $0.678(Z=-0.42)$ & $\begin{array}{c}701.33 \pm 688.16 \\
(0-6,324.29)\end{array}$ & $\begin{array}{c}712.11 \pm 592.75 \\
(0-3,238.00)\end{array}$ & $0.424(Z=-0.80)$ \\
\hline GAF score (mean \pm SD (range)) & $45.70 \pm 16.04(10-90)$ & $45.35 \pm 16.88(5-90)$ & $0.862(Z=-0.17)$ & $45.75 \pm 16.05(10-90)$ & $44.90 \pm 16.89(5-90)$ & $0.619(Z=-0.50)$ \\
\hline Females & $n=223-269^{b}$ & $n=75-92^{b}$ & & $n=229-273^{b}$ & $n=71-83^{b}$ & \\
\hline \multicolumn{7}{|l|}{ Target variables } \\
\hline Benzo use disorder (n (\%)) & $12(4.7)$ & $6(7.0)$ & $0.406\left(\chi^{2}=0.69\right)$ & $12(4.6)$ & $6(7.7)$ & $0.281\left(\chi^{2}=1.16\right)$ \\
\hline Anxiety composite score $(\text { mean } \pm S D)^{c, d}$ & $-0.02 \pm 0.77$ & $0.19 \pm 0.80$ & $0.028(F=4.91)$ & $-0.02 \pm 0.78$ & $0.21 \pm 0.77$ & $0.017(F=5.81)$ \\
\hline \multicolumn{7}{|l|}{ Sociodemographic } \\
\hline Age (at examination) (years) (mean \pm SD (range)) & $42.36 \pm 12.92(18-79)$ & $44.86 \pm 12.52(21-76)$ & $0.893(T=1.61)$ & $42.52 \pm 12.90(18-79)$ & $44.49 \pm 12.60(20-76)$ & $0.221(T=1.23)$ \\
\hline Education (years) $\left(\right.$ mean $\pm S D(\text { range) })^{e}$ & $14.56 \pm 3.95(7-31)$ & $14.12 \pm 3.54(8-27)$ & $0.447(Z=-0.76)$ & $14.52 \pm 3.94(7-31)$ & $14.13 \pm 3.62(8-27)$ & $0.454(Z=-0.75)$ \\
\hline Unemployment (n (\%)) & $76(31.9)$ & $20(25.0)$ & $0.243\left(\chi^{2}=1.37\right)$ & $74(30.5)$ & $20(28.2)$ & $0.712\left(\chi^{2}=0.14\right)$ \\
\hline \multicolumn{7}{|l|}{ Substance use } \\
\hline Alcohol use disorder according to DSM-IV-TR (n (\%)) & $59(22.4)$ & $14(15.7)$ & $0.178\left(\chi^{2}=1.82\right)$ & $60(22.4)$ & $13(16.0)$ & $0.219\left(x^{2}=1.51\right)$ \\
\hline Cannabis use disorder according to DSM-IV-TR (n (\%)) & $30(11.4)$ & $8(9.0)$ & $0.525\left(\chi^{2}=0.40\right)$ & $29(10.8)$ & $9(11.1)$ & $0.941\left(\chi^{2}=0.01\right)$ \\
\hline \multicolumn{7}{|l|}{ Clinical } \\
\hline PANSS positive score (mean \pm SD (range)) & $13.84 \pm 6.66(7-37)$ & $14.41 \pm 6.38(7-33)$ & $0.288(Z=-1.06)$ & $13.87 \pm 6.69(7-37)$ & $14.32 \pm 6.24(7-32)$ & $0.366(Z=-0.91)$ \\
\hline Chlorpromazine equivalents (mean \pm SD (range)) & $\begin{array}{c}636.37 \pm 776.51 \\
(0-7,375.00)\end{array}$ & $\begin{array}{c}704.59 \pm 762.50 \\
(0-4,370.00)\end{array}$ & $0.612(Z=-0.51)$ & $\begin{array}{c}634.40 \pm 771.10 \\
(0-7,375.00)\end{array}$ & $\begin{array}{c}718.83 \pm 788.67 \\
(0-4,370.00)\end{array}$ & $0.616(Z=-0.50)$ \\
\hline GAF score (mean \pm SD (range) $)$ & $46.34 \pm 19.42(8-90)$ & $44.22 \pm 17.59(12-84)$ & $0.435(Z=-0.78)$ & $46.22 \pm 19.25(8-90)$ & $44.88 \pm 18.07(12-84)$ & $0.645(Z=-0.46)$ \\
\hline
\end{tabular}

SD, standard deviation.

aFor statistical methods, Mann-Whitney $U$ or $\chi^{2}$ tests and for normally distributed variables $t$ tests were used. Bolded values: $p<0.05$.

${ }^{b}$ Because of missing data, sample sizes vary.

'Results after multiple imputations (10).

${ }^{\mathrm{d}}$ Analysis of covariance with age, positive symptoms (PANSS) and medication status (chlorpromazine equivalent) as covariates.

${ }^{e}$ Total years spent in education system; patients currently in school or educational training were excluded. 


\section{A}

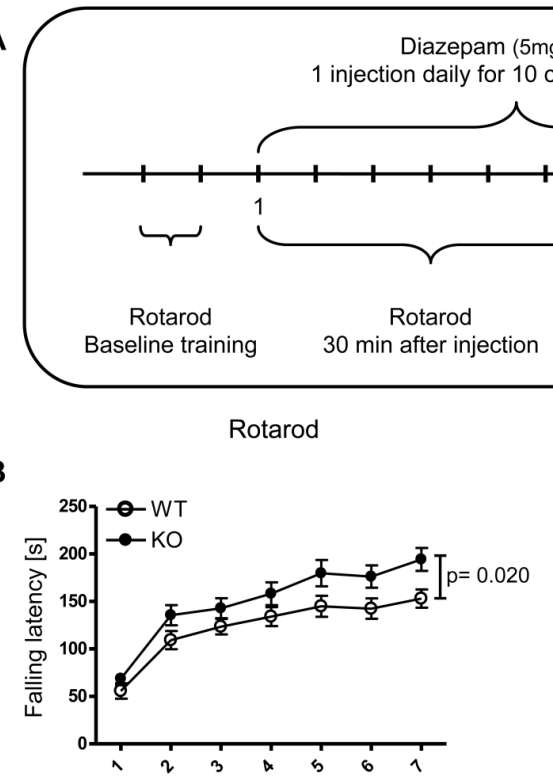

C
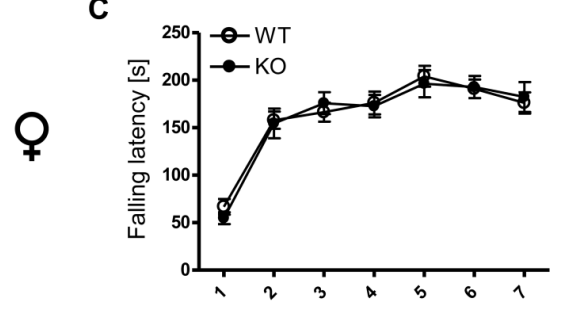

D

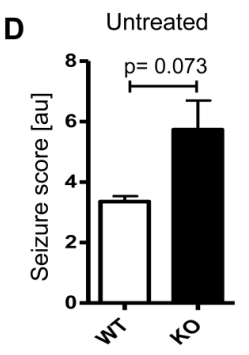

E

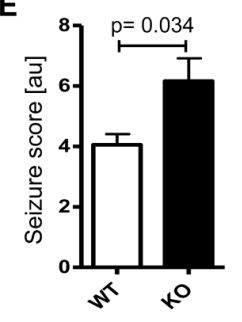

Flumazenil
$(15 \mathrm{mg} / \mathrm{kg} ; \mathrm{IP})$

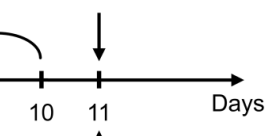

$\uparrow$

Pentylenetetrazole (50mg/ kg; IP) Seizure observation

Seizure propensity

F Diazepam

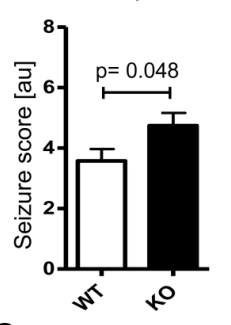

G

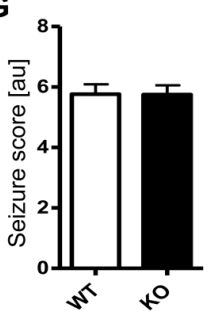

Figure 3. Diazepam tolerance and withdrawal in Baiap3 KO and WT mice. (A) Experimental design scheme. (B) Male diazepam-treated Baiap3 KO mice showed significantly faster improvement of performance on the rotarod, consistent with a more rapid development of tolerance to diazepam. (C) Rotarod performance of female mice was comparable between WT and KO. (D, E) Diazepam-naive Baiap3 KO mice display a higher PTZ-induced seizure propensity compared with WT (significant in females, strong tendency in males). (F, G) Flumazenil-induced diazepam withdrawal does not further increase PTZinduced seizure propensity in Baiap3 KO mice. Seizure propensity of female mice became comparable between genotypes, pointing to a ceiling effect. Numbers tested: males, $\mathrm{WT}=25, \mathrm{KO}=25$; females, $\mathrm{WT}=21, \mathrm{KO}=23$, except for $(\mathrm{D})$ and $(\mathrm{E})$, where males, $\mathrm{WT}=7$, $\mathrm{KO}=7$; females, $\mathrm{WT}=8 ; \mathrm{KO}=10$. Mann-Whitney $U$ test $(\mathrm{D}-\mathrm{G})$ and two-way repeatedmeasures ANOVA $(B, C)$, including Bonferroni, testing applied. Means \pm SEM are presented.

azepam withdrawal-related seizures, the susceptibility to PTZ-induced seizures was first evaluated in diazepam-naive mice. The seizure response of Baiap3 $\mathrm{KO}$ mice of both sexes to PTZ (50 mg/kg IP) was higher than that in WT animals, with the difference just failing to reach significance in males (Figures 3D, E). To assess the effect of genotype on benzodiazepine withdrawal, the diazepam antagonist flumazenil (15 mg/kg IP) was injected on d 11, after $10 \mathrm{~d}$ of daily diazepam treatment, immediately followed by PTZ injection (50 mg/kg IP) to trigger withdrawal seizures (Figure 3A). Upon flumazenil-induced diazepam withdrawal, the response to PTZ in male Baiap3 KO and WT mice did not differ appreciably from the one found in diazepam-naive mice of both genotypes (Figure 3F). In contrast, the genotypedependent differences in diazepam-naive females regarding seizure scores disappeared under conditions of diazepam withdrawal (Figure 3G), which could be explained by a ceiling effect. Thus, fe- male and male Baiap3 KO mice are more seizure-prone than their WT littermates, and this propensity is not further increased by benzodiazepine withdrawal.

\section{Drug Self-administration and Basic Behaviors Do Not Differ between Baiap3 Genotypes upon Chronic Addiction}

To assess whether Baiap3 KO mice, once addicted, would also be more likely to orally self-administer benzodiazepines, we performed an experiment on chronic midazolam addiction, where self-application was assessed after forced long-term exposure to escalating doses of midazolam (Supplementary Figure S6). We detected no genotype or gender differences in the clear preference for midazolam. Moreover, no genotype effects on body weight or basic behavior in the chronically addicted state were noted (Supplementary Figure S6). These data indicate that the Baiap3 genotype gender specifically affects the development of tolerance, that is, drug abuse at an early stage. In chronic addiction, genotype effects are no longer detectable.

\section{Lack of Homeostatic Adaptation to Diazepam in Baiap3 KO Hypothalamus Slices}

One hypothesis regarding predisposition to the development of addiction at the cellular level is an altered response to the addiction-inducing substance and its withdrawal. Because Baiap3 KO mice showed an increased seizure propensity and an altered development of tolerance to diazepam, we investigated whether lack of Baiap3 leads to a measurably altered response to diazepam treatment and withdrawal in neurons in vitro. Because Baiap3 expression is highest in the hypothalamus, we cultured organotypic hypothalamus slices prepared from male P5/P6 Baiap3 KO and WT animals in the presence of either $10 \mu \mathrm{mol} / \mathrm{L} \mathrm{di-}$ azepam or vehicle (DMSO) and recorded from neurons in the ventromedial hypothalamus in the presence of the diazepam antagonist flumazenil to 
mimic diazepam withdrawal conditions in vitro. We hypothesized that diazepam treatment would lead to a homeostatic adaptation in the $\mathrm{GABA}_{\mathrm{A}} \mathrm{R}$-mediated mIPSCs (Figure 4A) that would become apparent under diazepam withdrawal conditions. Although we observed no diazepam treatment-dependent differences that reached statistical significance, there was a significant genotypedependent effect under diazepam withdrawal conditions. Here, WT mIPSC amplitudes were $27 \%$ smaller (Figure 4B) and rise times 13\% longer than in $\mathrm{KO}$ neurons (Figure 4C), which is suggestive of a homeostatic adaptation to diazepam treatment in WT but not in $\mathrm{KO}$ slices. No significant differences in MIPSC decay times and frequencies were observed (Supplementary Table S2). Because the sudden withdrawal of diazepam should lead to an increase in overall network activity, we recorded action potential (AP) frequencies in ventromedial hypothalamus slices in the presence of flumazenil. Surprisingly, $\mathrm{KO}$ slices already showed significantly higher AP frequencies than WT slices under control conditions, with no further increase under diazepam withdrawal conditions. By contrast, in WT slices, we observed a significant increase in AP frequency under diazepam withdrawal conditions compared with vehicle-treated WT slices (Figure 4D). There was no significant difference in the resting membrane potentials (Figure $4 \mathrm{E}), \mathrm{AP}$ rise times, decay times and half-widths (Supplementary Table S2). IPSCs were recorded in the same cells at a holding potential of $-20 \mathrm{mV}$ to be able to isolate spontaneous $\mathrm{GABA}_{\mathrm{A}} \mathrm{R}$ mediated currents without drug application. In WT slices, we observed a significant effect of diazepam withdrawal, with an increase in IPSC amplitude and frequency compared with vehicletreated WT slices (Figures 4F, G), which is in keeping with the overall higher firing rate and which was not apparent in KO slices. In summary, these data show that neurons in Baiap3 KO hypothalamus slices have higher AP firing rates,
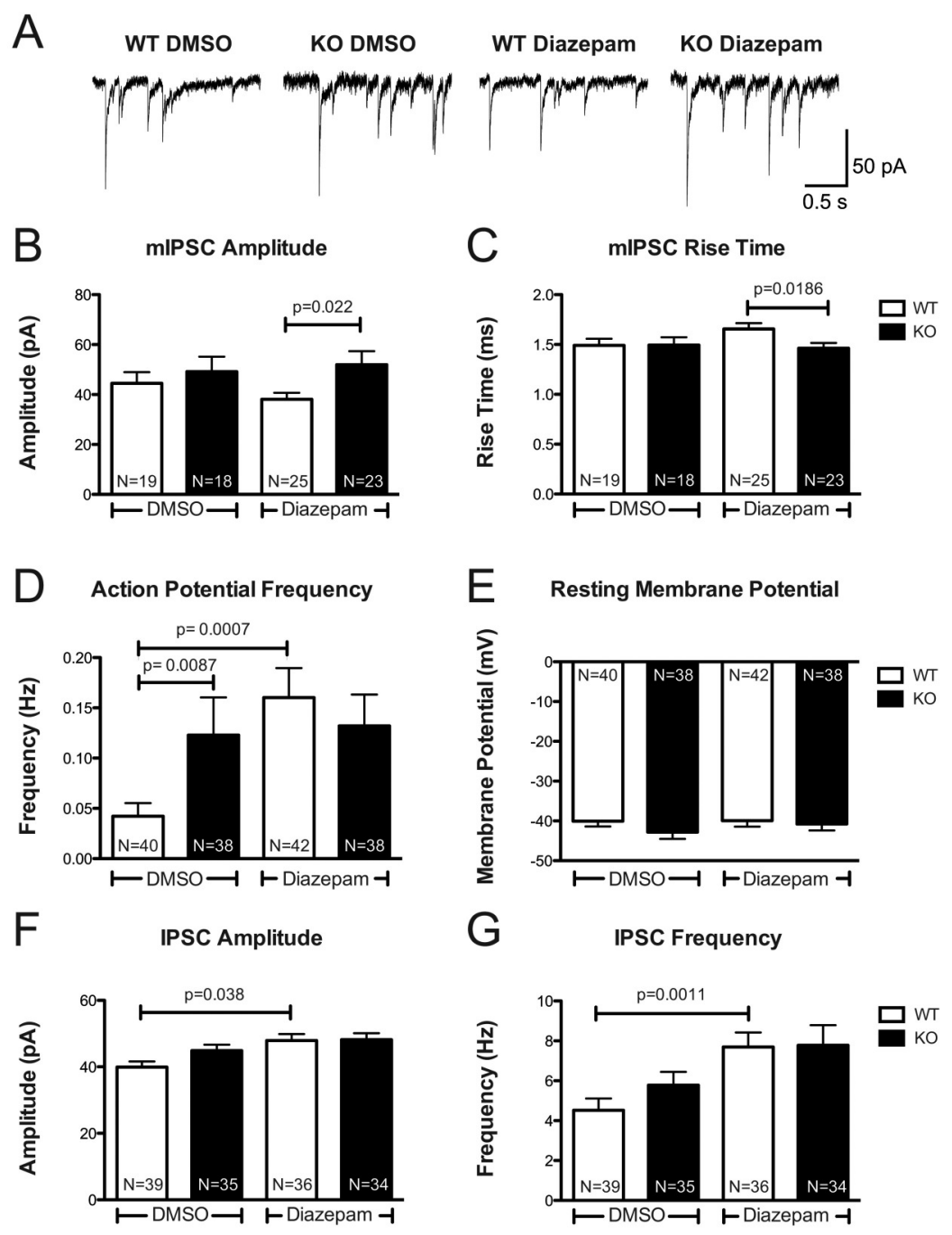

Figure 4. Increased basal network activity and lack of homeostatic adaptation to diazepam treatment in Baiap3 KO hypothalamus slices. (A) Sample traces of mIPSC recordings from WT and KO hypothalamus slices that were cultured in the presence of diazepam or under vehicle control conditions with DMSO. Under diazepam withdrawal conditions, Baiap3 WT mIPSC amplitudes were significantly smaller than in KO slices (B), and WT mIPSC rise times were longer than in KO slices (C). (D) Baiap3 WT slices showed an increase in AP frequency in response to diazepam withdrawal when compared with DMSO-treated WT slices, whereas no such increase was apparent for Baiap3 KO slices, which already showed an increased AP frequency under DMSO control conditions when compared with WT slices. (E) The resting membrane potential was not affected by experimental condition or Baiap3 genotype. IPSC amplitudes (F) and IPSC frequencies $(G)$ were increased in Baiap3 WT slices under diazepam withdrawal compared with DMSO-treated WT slices. Mann-Whitney U test was used for AP and IPSC frequencies; Student $t$ test was used for all other parameters. Means \pm SEM are presented.

likely consistent with the higher seizure propensity found in vivo, and that Baiap3 KO slices show no obvious homeostatic adaptation to diazepam treatment and withdrawal.

\section{DISCUSSION}

In this study, we identify two human BAIAP3 risk genotypes that are associated with anxiety in women and benzodiazepine use disorder in men. We fur- 
ther show that Baiap3 deficiency in mice leads to (i) elevated seizure propensity; (ii) increased anxiety in both genders, with a more pronounced effect in females; and (iii) a faster development of tolerance to benzodiazepines in male mice. In vitro analysis of hypothalamic slices revealed an increase in neuronal baseline activity in the absence of Baiap3. Withdrawal from chronic benzodiazepine application in vitro results in a genotype-specific response pattern.

To the best of our knowledge, no other genetic risk marker that is associated with anxiety and benzodiazepine abuse has been reported to date. We are aware that, pending replication in nonschizophrenic individuals, we cannot be sure that our findings can be applied to the general population. In spite of this limitation, our findings suggest a role for $B A I A P 3$ and potential interaction partners in the development of anxiety and drug dependence.

Unfortunately, similar data from samples of equally well-phenotyped healthy individuals or even other disease groups are not available. This is particularly true with regard to benzodiazepine abuse, since short-term exposure is a primary goal of controlled and medically surveyed indications. Even looking at other rare situations of long-term exposure (for example, intractable epilepsies), a homogeneous sample comparable to the GRAS collection would be difficult to imagine. In the GRAS sample, there are no differences between BAIAP3 genotypes regarding benzodiazepine exposure or daily dose (in case of exposure). This constellation allowed us to analyze the specific genetic risk of developing benzodiazepine use disorder in a setting close to an experimental condition.

Importantly, the present study was purely hypothesis-driven. Our hypotheses for performing a human phenotypebased genetic association study of $B A I A P 3$ were based on the anxiety phenotype we observed during basic behavioral characterization of Baiap3 $\mathrm{KO}$ mice as well as on the distinctive Baiap3 ex- pression pattern in brain, which includes regions relevant for addictive behaviors. We find that in humans, female carriers of the homozygous BAIAP3 risk genotypes (AA for SNP rs2235632; TT for SNP rs1132358) are more likely to meet criteria for an anxiety disorder, whereas male carriers of the same risk genotypes are more likely to fulfill criteria for benzodiazepine use disorder. Neither SNP was associated with schizophrenia in our case control analysis. Furthermore, no associations with substance use disorder other than benzodiazepine use disorder were observed. In general, both genetic linkage and candidate gene studies suffer from lack of replicability (12). However, in our study, the parallel identification of a gender-specific association of BAIAP3/Baiap3 with anxiety and an altered response to benzodiazepines in both mice and men, lends strong support to a causal link between BAIAP3 and the observed phenotypes.

As for other genetic variations associated with anxiety disorders $(11,12)$ or substance use disorders (13-15), the impact of BAIAP3 genotypes on anxiety disorders or benzodiazepine use disorder is likely to be small. However, the observed effects and their gender specificity (across two species) are intriguing. While we currently have no mechanistic insight into this gender specificity, part of the explanation may lie in the fact that Baiap3 is expressed in sexually dimorphic brain regions such as the hypothalamus, amygdala and the bed nucleus of the stria terminalis $(38,39)$.

Given the higher prevalence of both anxiety disorders and benzodiazepine use disorder in women $(9,40)$ the present findings were surprising at first glance, but the similarity of gender differences in mice and humans underlines their significance, encouraging follow-up work on this gender effect. Admittedly, the gender effects in humans may ultimately turn out to be less prominent, since the total number of individuals with benzodiazepine abuse in the GRAS sample is low, resulting in moderate significance levels only for men. It cannot be excluded that, in a larger sample, an association of benzodiazepine use disorder with the genotypes investigated here might reach significance for women as well. Furthermore, research focusing on gender differences and social desirability in self-reported anxiety suggests an underreporting of fear and distress in men (41-43). Therefore, our use of three self-reported measures in the calculation of the anxiety composite score might partly explain the lack of association of the BAIAP3 risk genotypes with anxiety in men. Nevertheless, gender differences in BAIAP3/Baiap3 genotype-phenotype associations most likely exist and are worth pursuing.

Benzodiazepines are positive allosteric modulators of $\mathrm{GABA}_{\mathrm{A}} \mathrm{R}$ and thus enhancers of inhibitory GABAergic neurotransmission. Their sedative, anti-convulsive and amnesic effects are largely mediated by the $\mathrm{GABA}_{\mathrm{A}} \mathrm{R} \alpha 1$ subunit, the anxiolytic effect by the $\alpha 2$ subunit and muscle relaxation by $\alpha 2, \alpha 3$ and $\alpha 5$ subunits (44). To date, no specific risk association of these obvious candidate genes has been identified. At present we have no evidence that would suggest that Baiap3 interacts with $\mathrm{GABA}_{\mathrm{A}} \mathrm{R}$ subunits. However, the increased seizure propensity observed in Baiap3 KO mice of both sexes, which is already apparent without prior diazepam treatment and withdrawal, is indicative of an altered balance of excitatory and inhibitory systems. Our comparison of neuronal firing rates in hypothalamus slices under baseline and diazepam withdrawal conditions uncovered an increase in basal network activity in the absence of Baiap3. This finding was unexpected, and although presently limited to the hypothalamus, is consistent with the increased seizure propensity observed in vivo. Even though we do not know whether the seizures observed in our PTZ-induction model originate in the subcortical regions that express Baiap3, subcortical epileptogenesis with origins in the hypothalamus is a feature seen in hypothalamic hamartomas (45), and the amygdala, which also expresses Baiap3, is known to play a key role in epileptogenesis (46). 
Interestingly, the human BAIAP3 gene is located on chromosome 16p13.3, which has been linked to electroencephalographic traits of idiopathic epilepsy syndromes $(47,48)$. We would thus argue that further investigation of BAIAP3 as a candidate gene for epilepsy-related phenotypes is warranted. Because we found that Baiap3 did not colocalize with markers of GABAergic or glutamatergic preand postsynapses to a significant degree (Supplementary Figures S1C-G), the increased seizure propensity in Baiap3 $\mathrm{KO}$ mice of both sexes and the altered response to benzodiazepines in males is unlikely to be due to a direct effect of Baiap3 at GABAergic or glutamatergic synapses.

The neuronal circuitry underlying the addictive properties of benzodiazepines is less well understood than their molecular mechanism of action. Unlike many other addictive substances, benzodiazepines do not appear to increase dopamine levels in the nucleus accumbens (49-51), although electrophysiological studies suggest that benzodiazepines increase firing of dopaminergic neurons in the VTA through disinhibition of these neurons via inhibition of nearby inhibitory interneurons (52,53). Additional mechanisms, such as neuroendocrine responses to benzodiazepine treatment, may play a critical role in the development of benzodiazepine use disorder (54). Furthermore, because expression of Baiap3 in both the VTA and in the nucleus accumbens is low (Allen Brain Atlas), a direct effect of Baiap3 on the mesolimbic dopamine pathway does not appear be the most likely explanation for the observed interaction between Baiap3 genotypes and the response to benzodiazepines. Instead, our findings support the interpretation that the altered response to benzodiazepines could be a consequence of a local or global change in neuronal excitability. Because all other members of the Munc13 protein family have been shown to be regulators of SNARE-mediated exocytosis $(25,29)$, Baiap3 may regulate the release of one or more modulatory neurotransmitters or neuropeptides that influence the balance between GABAergic and glutamatergic neurotransmission. Baiap3 immuoreactivity appears punctate (Supplementary Figures S1C-G) and may localize to peptidergic release sites, some of which may also contain VGLUT2 or Viaat. Although we presently cannot exclude the possibility that Baiap3 might have a postsynaptic function, given what is know about the function of all other members of the Munc13 protein family, we think that a pre-synaptic function is more likely. We can furthermore not exclude the possibility that alterations in the hypothalamicpituitary-adrenal axis may play a role in the anxiety phenotype or the altered response to benzodiazepines seen in Baiap3 $\mathrm{KO}$ mice. We are currently investigating whether Baiap3 is involved in regulating exocytosis of dense core vesicles and/or intracellular trafficking events that could influence neuropeptide release or extrasynaptic GABA $\mathrm{A}_{\mathrm{A}}$ R.

\section{CONCLUSION}

To conclude, BAIAP3 had not previously been considered a candidate gene for either psychiatric disorders or epilepsy. Our study links BAIAP3/Baiap3 genotypes to anxiety and an altered response to benzodiazepines in both mice and men and thus strongly argues for an involvement of BAIAP3 in these neuropsychiatrically relevant phenotypes. The identification of human genetic variations that influence the risk for the development of pathological phenotypes as well as the response to pharmacological treatments may pave the way for more efficient treatments with fewer side effects. Rodent models are usually only imperfect representations of human psychiatric conditions; however, the simultaneous identification of Baiap3 as a biomarker for anxiety and the response to benzodiazepines in mouse and humans suggests that Baiap3 $\mathrm{KO}$ mice will be a valuable tool in further elucidating the genetic, physiological and neuroanatomical underpinnings of anxiety disorders and benzodiazepine use disorder.

\section{ACKNOWLEDGMENTS}

We are indebted to all patients for their participation in the GRAS study and all collaborating GRAS centers for their support. We are grateful to all colleagues who contributed to the GRAS data collection. We would also like to thank Astrid Zeuch, Astrid Ohle and the members of the DNA sequencing core facility for excellent technical assistance. This work was supported by the Max Planck Society, the MaxPlanck-Förderstiftung, and the DFG Center for Nanoscale Microscopy and Molecular Physiology of the Brain.

\section{DISCLOSURE}

The authors declare that they have no competing interests as defined by Molecular Medicine, or other interests that might be perceived to influence the results and discussion reported in this paper.

\section{REFERENCES}

1. Kessler RC, et al. (2005) Lifetime prevalence and age-of-onset distributions of DSM-IV disorders in the National Comorbidity Survey Replication. Arch. Gen. Psychiatry. 62:593-602.

2. Pasche S. (2012) Exploring the comorbidity of anxiety and substance use disorders. Curr. Psychiatry Rep. 14:176-81.

3. Swendsen J, et al. (2010) Mental disorders as risk factors for substance use, abuse and dependence: results from the 10-year follow-up of the National Comorbidity Survey. Addiction. 105:1117-28.

4. Conway KP, Compton W, Stinson FS, Grant BF. (2006) Lifetime comorbidity of DSM-IV mood and anxiety disorders and specific drug use disorders: results from the National Epidemiologic Survey on Alcohol and Related Conditions. J. Clin. Psychiatry. 67:247-57.

5. Myrick H, Brady K. (2003) Current review of the comorbidity of affective, anxiety, and substance use disorders. Curr. Opin. Psychiatry. 16:261-70.

6. Bandelow B, et al. (2012) Guidelines for the pharmacological treatment of anxiety disorders, obsessive-compulsive disorder and posttraumatic stress disorder in primary care. Int. J. Psychiatry Clin. Pract. 16:77-84.

7. Baker AL, Thornton LK, Hiles S, Hides L, Lubman DI. (2012) Psychological interventions for alcohol misuse among people with co-occurring depression or anxiety disorders: a systematic review. J. Affect. Disord. 139:217-29.

8. Hettema JM, Neale MC, Kendler KS. (2001) A review and meta-analysis of the genetic epidemiology of anxiety disorders. Am. J. Psychiatry. 158:1568-78.

9. Hettema JM, Prescott CA, Myers JM, Neale MC, 
Kendler KS. (2005) The structure of genetic and environmental risk factors for anxiety disorders in men and women. Arch. Gen. Psychiatry. 62:182-9.

10. Ducci F, Goldman D. (2012) The genetic basis of addictive disorders. Psychiatr. Clin. North Am. 35:495-519.

11. Hovatta I, Barlow C. (2008) Molecular genetics of anxiety in mice and men. Ann. Med. 40:92-109.

12. Hamilton SP. (2009) Linkage and association studies of anxiety disorders. Depress. Anxiety. 26:976-83.

13. Wang JC, Kapoor M, Goate AM. (2012) The genetics of substance dependence. Annu. Rev. Genomics Hum. Genet. 13:241-61.

14. Gelernter J, Kranzler HR. (2010) Genetics of drug dependence. Dialogues Clin. Neurosci. 12:77-84.

15. Buckland PR. (2008) Will we ever find the genes for addiction? Addiction. 103:1768-76.

16. Marmorstein NR. (2012) Anxiety disorders and substance use disorders: different associations by anxiety disorder. J. Anxiety Disord. 26:88-94.

17. DeMartini KS, Carey KB. (2011) The role of anxiety sensitivity and drinking motives in predicting alcohol use: a critical review. Clin. Psychol. Rev. 31:169-77.

18. Zavos HM, Gregory AM, Eley TC. (2012) Longitudinal genetic analysis of anxiety sensitivity. Dev. Psychol. 48:204-12.

19. Kushner MG, Thuras P, Abrams K, Brekke M, Stritar L. (2001) Anxiety mediates the association between anxiety sensitivity and coping-related drinking motives in alcoholism treatment patients. Addict. Behav. 26:869-85.

20. Baldwin DS, Allgulander C, Bandelow B, Ferre F, Pallanti S. (2012) An international survey of reported prescribing practice in the treatment of patients with generalised anxiety disorder. World J. Biol. Psychiatry. 13:510-6.

21. Canteras NS, Resstel LB, Bertoglio LJ, Carobrez Ade P, Guimaraes FS. (2010) Neuroanatomy of anxiety. Curr. Top. Behav. Neurosci. 2:77-96.

22. Gratacos M, et al. (2007) Candidate genes for panic disorder: insight from human and mouse genetic studies. Genes Brain Behav. 6 (Suppl. 1):2-23.

23. Koch H, Hofmann K, Brose N. (2000) Definition of Munc13-homology-domains and characterization of a novel ubiquitously expressed Munc13 isoform. Biochem. J. 349:247-53.

24. Shiratsuchi T, et al. (1998) Cloning and characterization of BAP3 (BAI-associated protein 3), a C2 domain-containing protein that interacts with BAI1. Biochem. Biophys. Res. Commun. 251:158-65.

25. Varoqueaux F, et al. (2002) Total arrest of spontaneous and evoked synaptic transmission but normal synaptogenesis in the absence of Munc13-mediated vesicle priming. Proc. Natl. Acad. Sci. U. S. A. 99:9037-42.

26. Gorman JM, Kent JM, Sullivan GM, Coplan JD. (2000) Neuroanatomical hypothesis of panic disorder, revised. Am. J. Psychiatry. 157:493-505.

27. Gross CT, Canteras NS. (2012) The many paths to fear. Nat. Rev. Neurosci. 13:651-8.

28. Wojcik SM, Brose N. (2007) Regulation of mem- brane fusion in synaptic excitation-secretion coupling: speed and accuracy matter. Neuron. 55:11-24.

29. Feldmann J, et al. (2003) Munc13-4 is essential for cytolytic granules fusion and is mutated in a form of familial hemophagocytic lymphohistiocytosis (FHL3). Cell. 115:461-73.

30. Shirakawa R, et al. (2004) Munc13-4 is a GTPRab27-binding protein regulating dense core granule secretion in platelets. J. Biol. Chem. 279:10730-7.

31. Begemann M, et al. (2010) Modification of cognitive performance in schizophrenia by complexin 2 gene polymorphisms. Arch. Gen. Psychiatry. 67:879-88.

32. Ribbe K, et al. (2010) The cross-sectional GRAS sample: a comprehensive phenotypical data collection of schizophrenic patients. BMC Psychiatry. 10:91.

33. Ferraro TN, et al. (1999) Mapping loci for pentylenetetrazol-induced seizure susceptibility in mice. J. Neurosci. 19:6733-9.

34. American Psychiatric Association (APA). (2000) Diagnostic and Statistical Manual of Mental Disorders: DSM-IV-TR. 4th ed., text revision. Washington (DC): APA. $992 \mathrm{pp}$.

35. Kay SR, Fiszbein A, Opler LA. (1987) The positive and negative syndrome scale (PANSS) for schizophrenia. Schizophr. Bull. 13:261-76.

36. Cronbach LJ. (1951) Coefficient alpha and the internal structure of tests. Psychometrika. 16:297-334

37. Rubin DB. (1987) Multiple Imputation for Non-Response in Surveys. New York: John Wiley \& Sons.

38. Stefanova N, Ovtscharoff W. (2000) Sexual dimorphism of the bed nucleus of the stria terminalis and the amygdala. Adv. Anat. Embryol. Cell Biol. 158:III-X, 1-78.

39. Qureshi IA, Mehler MF. (2010) Genetic and epigenetic underpinnings of sex differences in the brain and in neurological and psychiatric disease susceptibility. Prog. Brain Res. 186:77-95.

40. Neutel CI. (2005) The epidemiology of long-term benzodiazepine use. Int. Rev. Psychiatry. 17:189-97.

41. Pierce KA, Kirkpatrick DR. (1992) Do men lie on fear surveys? Behav. Res. Ther. 30:415-8.

42. McLean CP, Anderson ER. (2009) Brave men and timid women? A review of the gender differences in fear and anxiety. Clin. Psychol. Rev. 29:496-505.

43. Stoyanova M, Hope DA. (2012) Gender, gender roles, and anxiety: perceived confirmability of self report, behavioral avoidance, and physiological reactivity. J. Anxiety Disord. 26:206-14.

44. Tan KR, Rudolph U, Luscher C. (2011) Hooked on benzodiazepines: GABAA receptor subtypes and addiction. Trends Neurosci. 34:188-97.

45. Fenoglio KA, et al. (2007) Hypothalamic hamartoma: basic mechanisms of intrinsic epileptogenesis. Semin. Pediatr. Neurol. 14:51-9.

46. Aroniadou-Anderjaska V, Fritsch B, Qashu F, Braga MF. (2008) Pathology and pathophysiology of the amygdala in epileptogenesis and epilepsy. Epilepsy Res. 78:102-16.

47. Pinto D, et al. (2005) Genome-wide linkage scan of epilepsy-related photoparoxysmal electroencephalographic response: evidence for linkage on chromosomes 7q32 and 16p13. Hum. Mol. Genet. 14:171-8.

48. de Kovel CG, et al. (2010) Whole-genome linkage scan for epilepsy-related photosensitivity: a mega-analysis. Epilepsy Res. 89:286-94.

49. Zetterstrom T, Fillenz M. (1990) Local administration of flurazepam has different effects on dopamine release in striatum and nucleus accumbens: a microdialysis study. Neuropharmacology. 29:129-34.

50. Invernizzi R, Pozzi L, Samanin R. (1991) Release of dopamine is reduced by diazepam more in the nucleus accumbens than in the caudate nucleus of conscious rats. Neuropharmacology. 30:575-8.

51. Finlay JM, Damsma G, Fibiger HC. (1992) Benzodiazepine-induced decreases in extracellular concentrations of dopamine in the nucleus accumbens after acute and repeated administration. Psychopharmacology (Berl.). 106:202-8.

52. Tan KR, et al. (2010) Neural bases for addictive properties of benzodiazepines. Nature. 463:769-74.

53. O'Brien DP, White FJ. (1987) Inhibition of nondopamine cells in the ventral tegmental area by benzodiazepines: relationship to A10 dopamine cell activity. Eur. J. Pharmacol. 142:343-54.

54. Heberlein A, Bleich S, Kornhuber J, Hillemacher T. (2008) Neuroendocrine pathways in benzodiazepine dependence: new targets for research and therapy. Hum. Psychopharmacol. 23:171-81. 
Supplemental Data

\section{Genetic Markers of a Munc 13 Protein Family Member, BAIAP3, Are Gender Specifically Associated with Anxiety and Benzodiazepine Abuse in Mice and Humans}

Sonja M Wojcik, ${ }^{1^{*}}$ Martesa Tantra, ${ }^{2,3^{*}}$ Beata Stepniak, ${ }^{2^{*}}$ Kwun-nok M Man, ${ }^{1,3}$ Katja Müller-Ribbe, Martin Begemann, ${ }^{2}$ Anes Ju, ${ }^{2}$ Sergi Papiol, ${ }^{2,3}$ Anja Ronnenberg, ${ }^{2}$ Artem Gurvich, ${ }^{2}$ Yong Shin, ${ }^{1,4}$ Iris Augustin, ${ }^{1,5}$ Nils Brose, ${ }^{1,3}$ and Hannelore Ehrenreich ${ }^{2,3}$

Online address: http://www.molmed.org

The Feinstein Institute North
for Medical Research

Supplementary Table S1. Benzodiazepine use (\%) and dose (lorazepam equivalents) in GRAS patients sorted by BAIAP3 genotypes.

\begin{tabular}{|c|c|c|c|c|c|c|c|c|}
\hline \multirow[b]{2}{*}{ Males (GRAS sample) } & \multicolumn{4}{|c|}{ BAIAP3 rs2235632 } & \multicolumn{4}{|c|}{ BAIAP3 rs 1132358} \\
\hline & GC & $A G$ & AA & $\begin{array}{c}\text { pvalue } \\
\left(\chi^{2} \text { value }\right)^{a}\end{array}$ & $\mathrm{CC}$ & $\mathrm{TC}$ & TT & $\begin{array}{c}\text { pvalue } \\
\left(\chi^{2} \text { value }\right)^{a}\end{array}$ \\
\hline Receiving benzodiazepines, No. (\%) ${ }^{b}$ & $\begin{array}{c}\mathrm{N}=181 \\
22(12.2) \\
\mathbf{N}=\mathbf{2} 1^{\mathrm{d}}\end{array}$ & $\begin{array}{c}\mathrm{N}=\mathbf{3 5 1} \\
57(16.2) \\
\mathrm{N}=\mathbf{5 2}^{\mathrm{d}}\end{array}$ & $\begin{array}{c}\mathrm{N}=187 \\
30(16.0) \\
\mathrm{N}=30\end{array}$ & $.427\left(\chi^{2}=1.70\right)$ & $\begin{array}{c}\mathrm{N}=184 \\
20(10.9) \\
\mathrm{N}=19^{\mathrm{d}}\end{array}$ & $\begin{array}{c}\mathrm{N}=347 \\
57(16.4) \\
\mathrm{N}=52^{\mathrm{d}}\end{array}$ & $\begin{array}{c}\mathrm{N}=175 \\
30(17.1) \\
\mathrm{N}=30\end{array}$ & $.165\left(\chi^{2}=3.60\right)$ \\
\hline Benzodiazepine dose, $\mathrm{mg}$, Mean $\pm S D$ b, & $3.14 \pm 4.14$ & $2.64 \pm 3.11$ & $3.04 \pm 3.31$ & $.833\left(\chi^{2}=0.37\right)$ & $2.95 \pm 4.31$ & $2.67 \pm 3.11$ & $3.00 \pm 3.32$ & $.979\left(\chi^{2}=0.04\right)$ \\
\hline Females (GRAS sample) & GC & $A G$ & AA & $\begin{array}{c}\text { p value } \\
\left(\chi^{2} \text { value }\right)^{a}\end{array}$ & $\mathrm{CC}$ & $\mathrm{TC}$ & TT & $\begin{array}{c}\text { p value } \\
\left(\chi^{2} \text { value }\right)^{a}\end{array}$ \\
\hline Receiving benzodiazepines, No. (\%) ${ }^{b}$ & $\begin{array}{c}\mathrm{N}=101 \\
28(27.7) \\
\mathrm{N}=27^{\mathrm{d}}\end{array}$ & $\begin{array}{c}\mathrm{N}=167 \\
34(20.4) \\
\mathrm{N}=3^{\mathrm{d}}\end{array}$ & $\begin{array}{c}\mathrm{N}=92 \\
23(25.0) \\
\mathrm{N}=21^{\mathrm{d}}\end{array}$ & $.363\left(\chi^{2}=2.02\right)$ & $\begin{array}{c}\mathrm{N}=105 \\
30(28.6) \\
\mathrm{N}=29^{\mathrm{d}}\end{array}$ & $\begin{array}{c}\mathrm{N}=167 \\
34(20.4) \\
\mathrm{N}=33^{\mathrm{d}}\end{array}$ & $\begin{array}{c}\mathrm{N}=83 \\
20(24.1) \\
\mathrm{N}=18^{\mathrm{d}}\end{array}$ & $.298\left(\chi^{2}=2.42\right)$ \\
\hline Benzodiazepine dose, $\mathrm{mg}$, Mean $\pm S D$ b, c & $2.51 \pm 1.51$ & $2.13 \pm 2.35$ & $2.33 \pm 2.28$ & $.227\left(\chi^{2}=2.97\right)$ & $2.44 \pm 1.49$ & $2.08 \pm 2.37$ & $2.52 \pm 2.40$ & $.234\left(\chi^{2}=2.90\right)$ \\
\hline
\end{tabular}

Supplementary Table S2. The mIPSC frequencies and decay times and the AP rise times, decay times and half-width were not affected by experimental condition or Baiap3 genotype.

\begin{tabular}{lcccc}
\hline & WT DMSO & KO DMSO & WT Diazepam & KO Diazepam \\
\hline mIPSC Decay Time & $20.27 \pm 4.35 \mathrm{~ms}$ & $21.01 \pm 4.94 \mathrm{~ms}$ & $21.40 \pm 4.22 \mathrm{~ms}$ & $20.36 \pm 4.21 \mathrm{~ms}$ \\
mIPSC Frequency & $3.99 \pm 3.26 \mathrm{~Hz}$ & $5.59 \pm 5.78 \mathrm{~Hz}$ & $4.43 \pm 3.74 \mathrm{~Hz}$ & $3.61 \pm 2.80 \mathrm{~Hz}$ \\
AP Rise Time & $0.97 \pm 0.36 \mathrm{~ms}$ & $1.02 \pm 0.45 \mathrm{~ms}$ & $1.05 \pm 0.37 \mathrm{~ms}$ & $1.12 \pm 0.34 \mathrm{~ms}$ \\
AP Decay Time & $1.17 \pm 0.17 \mathrm{~ms}$ & $1.14 \pm 0.24 \mathrm{~ms}$ & $1.14 \pm 0.24 \mathrm{~ms}$ & $1.21 \pm 0.36 \mathrm{~ms}$ \\
AP Half-Width & $1.22 \pm 0.19 \mathrm{~ms}$ & $1.22 \pm 0.29 \mathrm{~ms}$ & $1.20 \pm 0.26 \mathrm{~ms}$ & $1.27 \pm 0.39 \mathrm{~ms}$ \\
\hline
\end{tabular}

Mann-Whitney U test for mIPSC frequencies and Student's t-test for all other parameters. Mean \pm SD presented. 
A
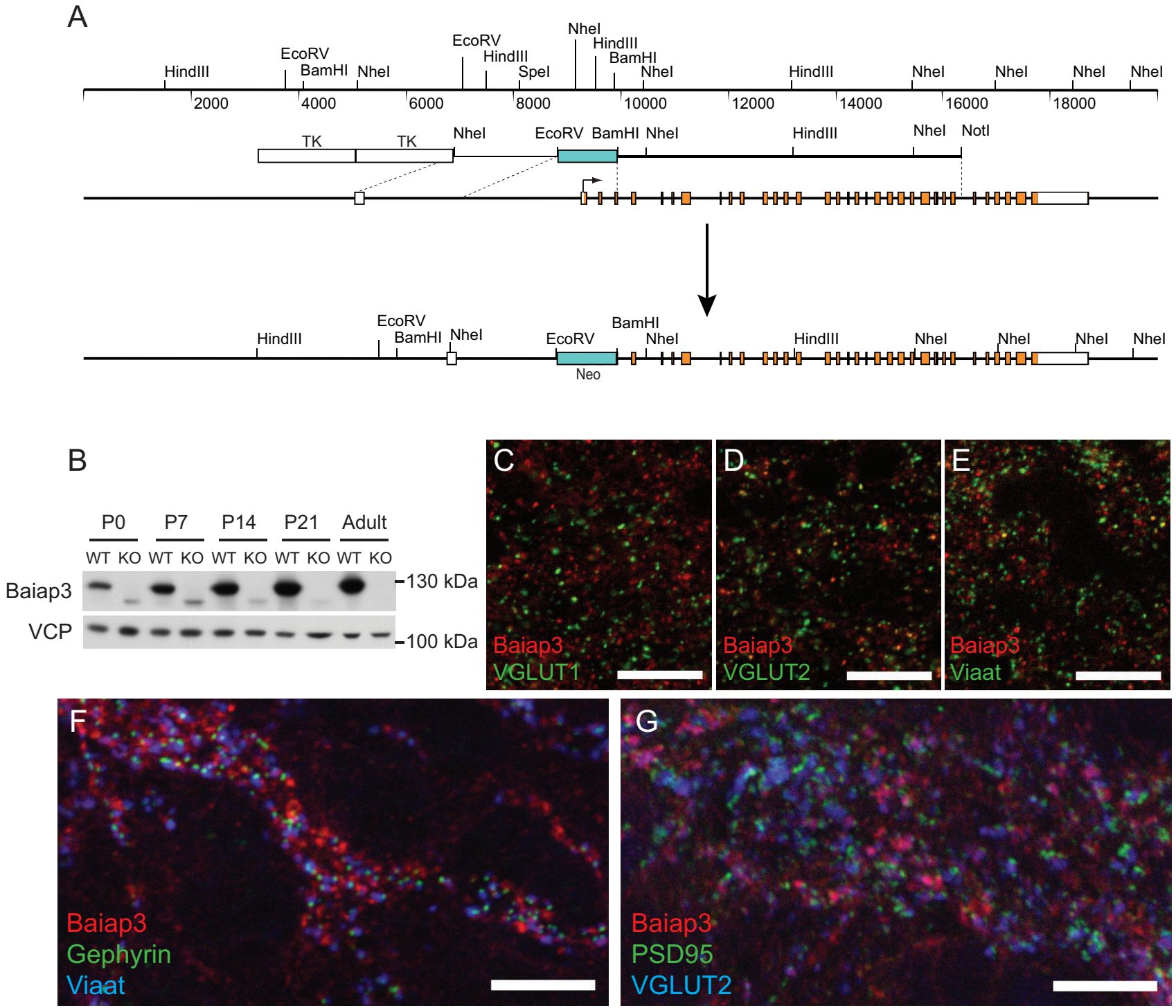

Supplementary Figure S1. Targeting strategy and expression of mouse Baiap3. (A) Targeting strategy for the mouse Baiap3 gene. The first 3 coding exons were replaced with a neomycin selection cassette ( $\mathrm{NeO}$ ). The targeting vector further contained 2 thymidine kinase cassettes (TK) for negative selection. (B) Western blot analysis of the expression of Baiap3, in P0, P7, P14, P21 and adult Baiap3 WT and KO animals. In Baiap3 KO pups, we detected a truncated Baiap3-immunoreactive product that decreased after P7 and was not detectable in adult mice. An antibody to the valosin-containing-protein (VCP) ATPase was used as a loading control. (C-E) Immunostaining analyses in the hypothalamic medial preoptic area show that Baiap3 largely does not co-localize with markers of glutamatergic and GABAergic synapses. (C) Baiap3 (red) shows no co-localization with the vesicular glutamate tranporter (VGLUT)1 (green). (D) Baiap3 (red) shows only limited co-localization with VGLUT2 (green) (E) Baiap3 (red) shows only limited co-localization with the vesicular inhibitory amino acid transporter (Viaat) (green). (F) In organotypic hypothalamus slices Baiap3 (red) does not show significant co-localization with presynaptic (Viaat, blue) or post-synaptic (Gephyrin, green) markers of GABAergic synapses or (G) with pre-synaptic (VGLUT2, blue) or postsynaptic (postsynaptic-density-protein 95, green) markers of glutamatergic synapses. Scale bars equal 10um. 


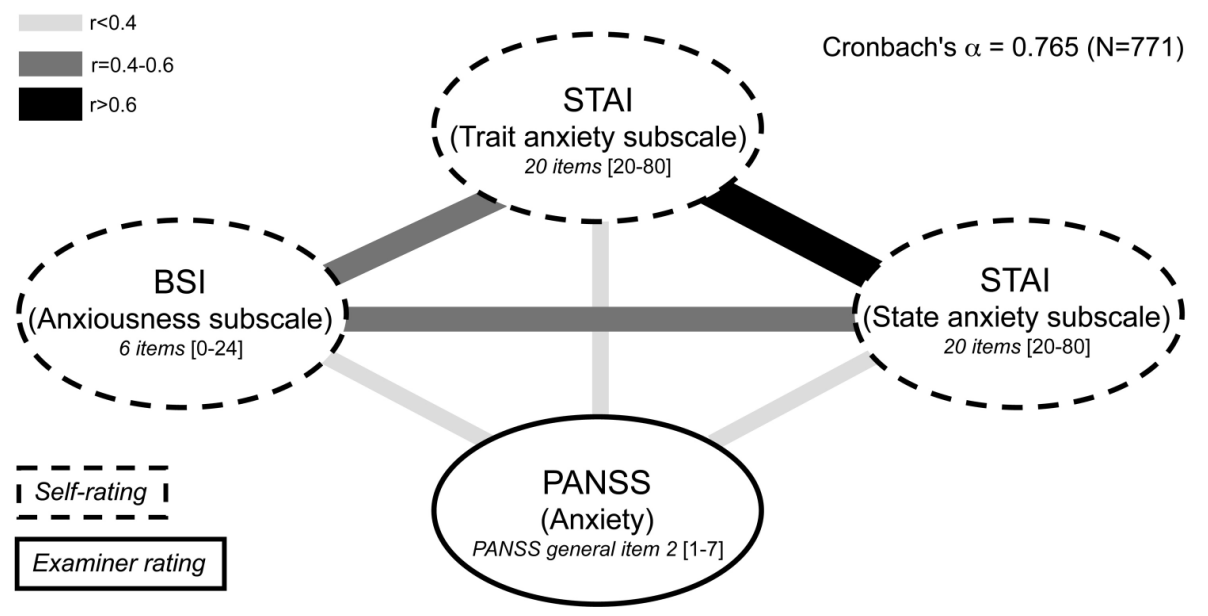

Supplementary Figure S2. Anxiety composite score. Shown are variables composing the anxiety composite score, their intercorrelations and internal consistency. Pearson's correlation coefficients and Cronbach's $\alpha$ given.

A

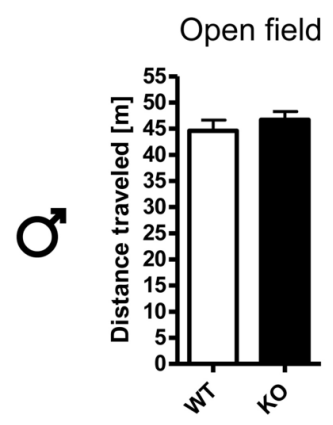

B

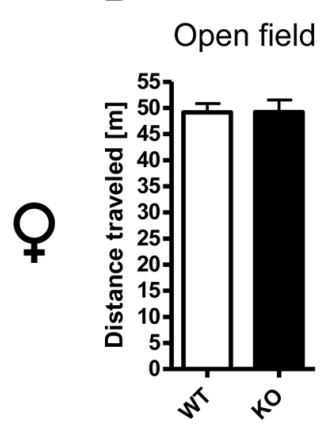

C

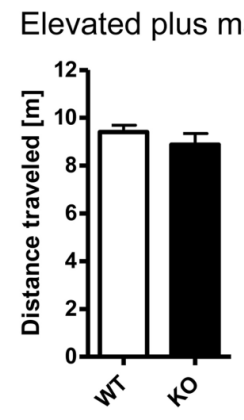

D

Elevated plus maze

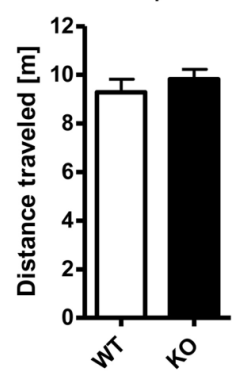

E

Hole board

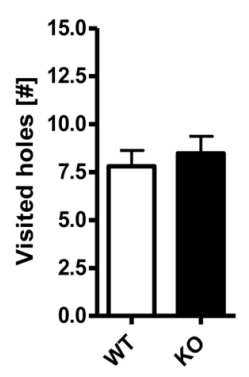

$\mathbf{F}$

Hole board

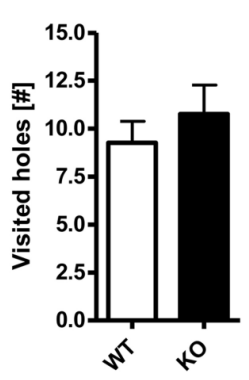

G

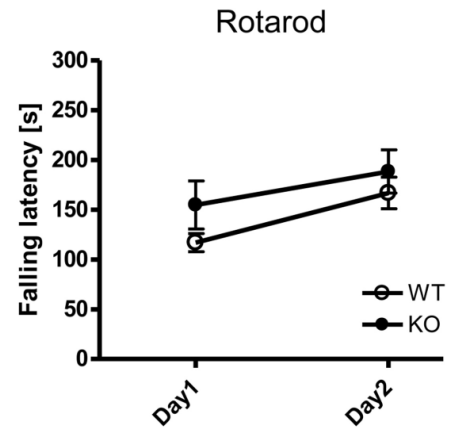

H

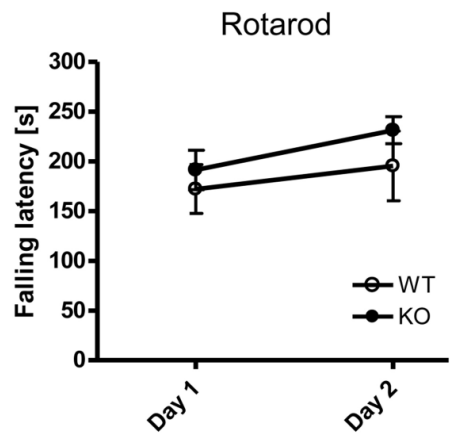

I

Body weight

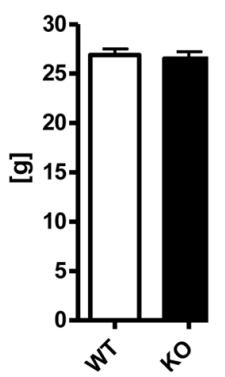

J

Body weight

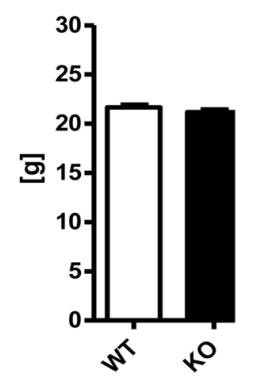

Supplementary Figure S3. Baiap3 genotype does not affect activity level, exploratory behavior, motor function or body weight. $(A, B)$ The distance traveled during a 7-min session in the open field and (C,D) 5-min session in the elevated plus-maze was comparable among genotypes and genders. $(E, F)$ Exploratory behavior measured in the hole board, $(G, H)$ motor coordination and learning, evaluated by rotarod, as well as $(\mathrm{I}, \mathrm{J})$ body weight were comparable for both genders between Baiap3 KO and WT littermates. Numbers tested: males, WT=16-25, $K O=16-25$; females, $W T=18-23, K O=10-28$. Mann-Whitney $U$ test $(A-F, I, J)$ and 2-way repeated measures ANOVA $(G, H)$, including Bonferroni testing, were applied. Mean \pm s.e.m. presented. 
A

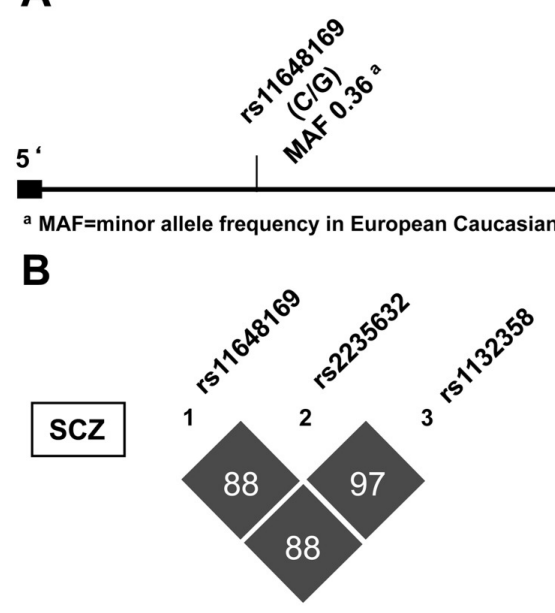

C

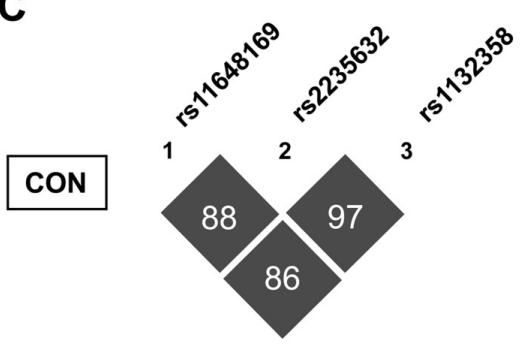

BAIAP3 (15.8kb, 16p13.3)

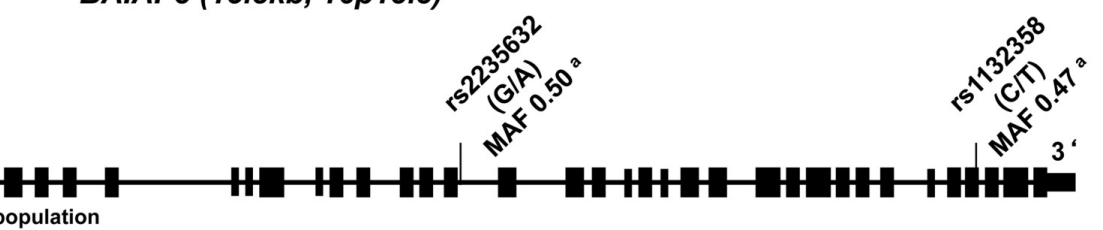

D

\begin{tabular}{|c|c|c|c|c|c|c|}
\hline \multirow[t]{2}{*}{ BAIAP3 } & & \multicolumn{3}{|c|}{$\begin{array}{l}\text { Genotypic frequencies \% } \\
\text { (N numbers) }\end{array}$} & \multirow[t]{2}{*}{$\begin{array}{l}p \text { value a } \\
\left(\chi^{2} \text { value }\right)\end{array}$} & \multirow[t]{2}{*}{$\begin{array}{l}\text { pvalue } \\
\text { HWE }\end{array}$} \\
\hline & & Cc & GC & GG & & \\
\hline \multirow[t]{3}{*}{ rs11648169 } & SCZ & $\begin{array}{l}43.2 \\
(467)\end{array}$ & $\begin{array}{l}44.2 \\
(478)\end{array}$ & $\begin{array}{l}12.7 \\
(137)\end{array}$ & $\begin{array}{c}0.792 \\
\left(\chi^{2}=0.47\right)\end{array}$ & 0.395 \\
\hline & CON & $\begin{array}{l}43.4 \\
(495)\end{array}$ & $\begin{array}{l}44.9 \\
(513)\end{array}$ & $\begin{array}{l}11.7 \\
(134)\end{array}$ & & 0.950 \\
\hline & & GG & AG & AA & & \\
\hline \multirow[t]{3}{*}{ rs2235632 } & SCZ & $\begin{array}{l}26.2 \\
(284)\end{array}$ & $\begin{array}{l}48.1 \\
(523)\end{array}$ & $\begin{array}{l}25.7 \\
(279)\end{array}$ & $\begin{array}{c}0.440 \\
\left(\chi^{2}=1.64\right)\end{array}$ & 0.225 \\
\hline & CON & $\begin{array}{l}26.6 \\
(304)\end{array}$ & $\begin{array}{l}50.0 \\
(571)\end{array}$ & $\begin{array}{l}23.4 \\
(267)\end{array}$ & & 0.971 \\
\hline & & cc & TC & TT & & \\
\hline \multirow[t]{2}{*}{ rs1132358 } & SCZ & $\begin{array}{l}27.3 \\
(292)\end{array}$ & $\begin{array}{l}48.6 \\
(519)\end{array}$ & $\begin{array}{l}24.1 \\
(258)\end{array}$ & $\begin{array}{c}0.178 \\
\left(\chi^{2}=3.46\right)\end{array}$ & 0.360 \\
\hline & CON & $\begin{array}{l}28.7 \\
(328)\end{array}$ & $\begin{array}{c}50.4 \\
(576)\end{array}$ & $\begin{array}{l}20.9 \\
(238)\end{array}$ & & 0.611 \\
\hline
\end{tabular}

a Comparison of genotype distributions between GRAS sample (SCZ) and healthy controls (CON). b Hardy-Weinberg-equilibrium.

Supplementary Figure S4. BAIAP3 genotyping strategy and case-control analyses. (A) Location of the selected single nucleotide polymorphisms (SNPs) in the BAIAP3 gene. Kb, kilobases. (B) Linkage disequilibrium map for $\mathrm{N}=1086$ schizophrenic and schizoaffective patients and (C) Linkage disequilibrium map for $\mathrm{N}=1142$ healthy blood donors indicating a high degree of linkage between the 3 selected SNPs in both groups. (D) Case-control comparisons reveal a similar distribution of the BAIAP3 SNP genotypes for patients and healthy individuals, thus excluding the selected BAIAP3 markers as risk factors for schizophrenia.

A SNP rs2235632
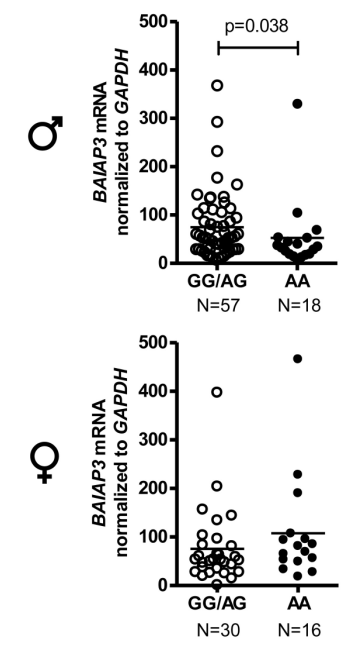

B

SNP rs 1132358
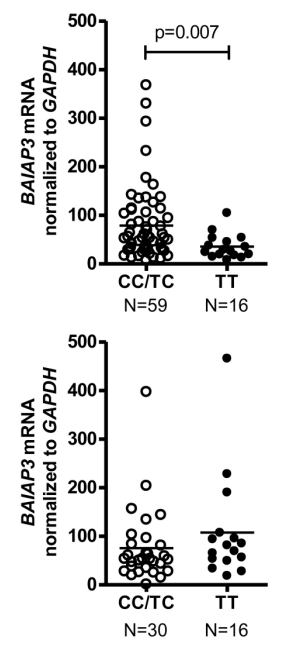

Supplementary Figure S5. BAIAP3 mRNA expression in PBMCs. (A) SNP rs2235632: The risk genotype AA is associated with lower BAIAP3 mRNA levels in male but not in female patients. (B) SNP rs 1 132358: The risk genotype $T$ is associated with lower BAIAP3 mRNA levels in male but not in female patients. Mann-Whitney U test applied, due to non-normal data distribution (A,B). 


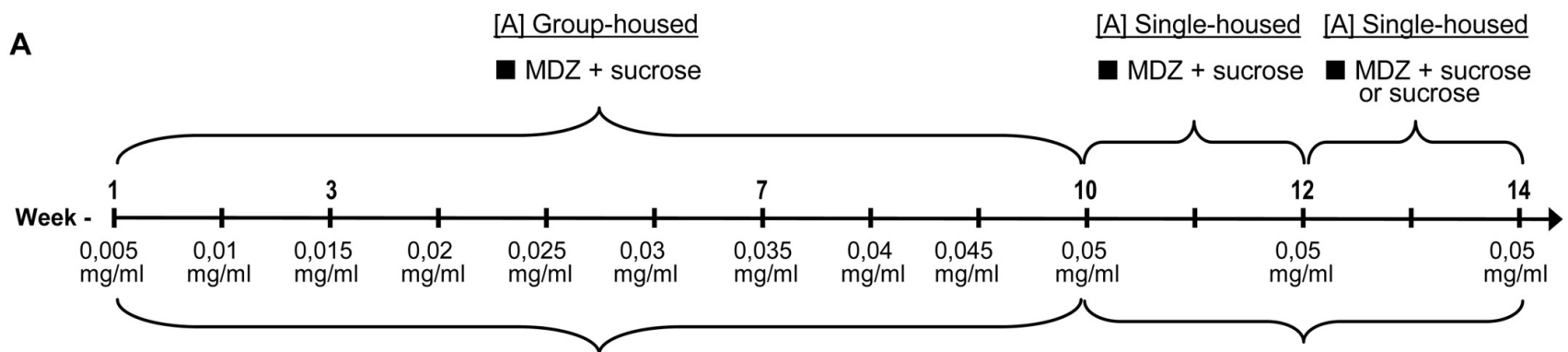

[B] Group-housed

MDZ + sucrose, or sucrose
[B] Group-housed

MDZ + sucrose, or sucrose

Behavioral testing under chronic MDZ

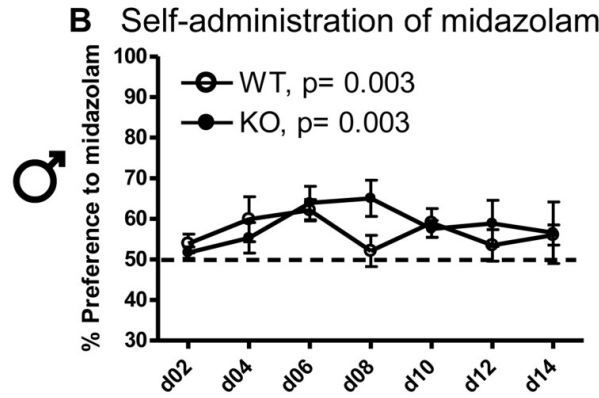

C Self-administration of midazolam

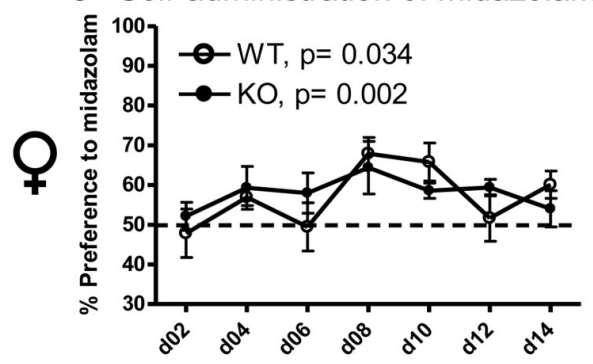

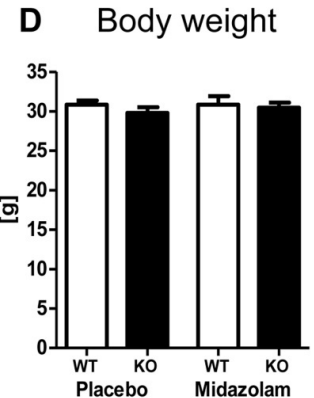

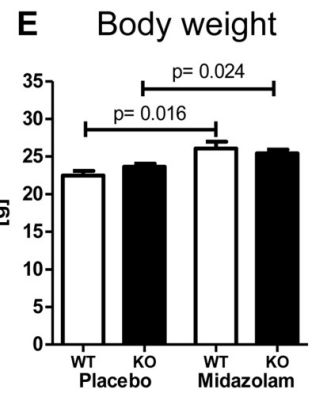

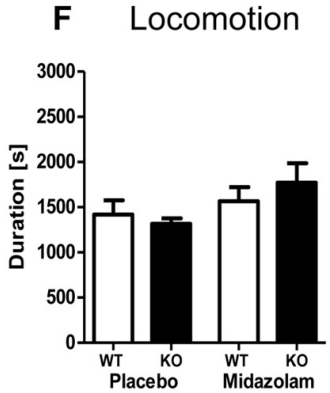

G Locomotion

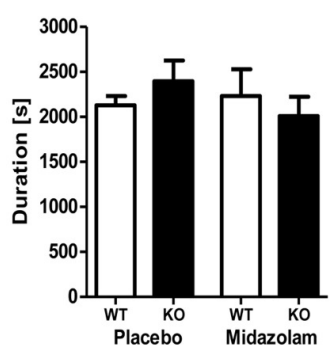

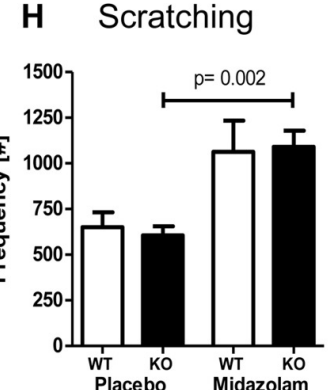

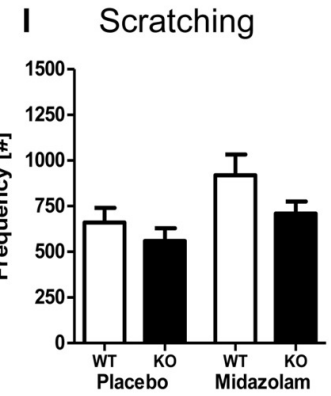

Supplementary Figure S6. High oral self-administration of midazolam (as readout of addiction), body weight, and basic behaviors in the chronic addicted state are not affected by Baiap3 genotype. (A) Experimental design scheme. (B, C) Baiap3 KO and WT mice of both genders displayed significant and comparable preference for midazolam over sucrose. (D,E) Chronic midazolam intake did not affect body weight of male, but increased that of female mice independently of genotype. $(F, G)$ Locomotion duration in LABORAS ${ }^{T M}$ remained unaffected across genotypes and genders. (H,I) Scratching frequency in LABORAS ${ }^{\mathrm{TM}}$ tended to be increased upon chronic high-dose midazolam across genotypes and genders. Numbers tested: males, $W T=6-8, K O=10-16$; females, WT=6-10, $K O=10-13$, except for (B) and (C), males, $\mathrm{WT}=8, \mathrm{KO}=4$; females, $\mathrm{WT}=5 ; \mathrm{KO}=4$; 2-way repeated measures ANOVA (B,C) as well as 2-way ANOVA (D-I), including Bonferroni testing, where applicable. Mean \pm s.e.m. presented. 


\section{MOUSE MODEL TARGETING A TRANSCRIPTIONAL REGULATOR, MECP2 (I):}

\section{Mild overexpression of Mecp2 in mice causes a higher susceptibility toward seizures}

\section{a. Overview of project II}

An intriguing finding about the transcriptional regulator MECP2 (methyl-CpG-bindingprotein 2) gene is that the loss-of-function mutations are attributable to Rett syndrome (RTT) (Amir, Van den Veyver et al. 1999), while the gain-of-function causes another neurological disorder, termed MECP2 duplication syndrome (Ramocki, Tavyev et al. 2010), indicating that MECP2 is dosage-sensitive (Collins, Levenson et al. 2004; Ramocki, Tavyev et al. 2010; Chao and Zoghbi 2012). This is also evident from mouse studies. Previously, a transgenic mouse model that overexpresses Mecp2 at twice the level of endogenous Mecp2 was generated and characterized (Collins, Levenson et al. 2004). The phenotypes of the mice are reminiscent of the human MECP2 duplication syndrome, with the onset of the phenotypes at around 10 weeks of age, when these mice started showing stereotyped and repetitive movements, motor dysfunctions, hypoactivity, ataxia, spasticity, epilepsy and $30 \%$ of them died by 1 year of age (Collins, Levenson et al. 2004). Interestingly, in another study, evaluation of a Mecp2 $2^{f l o x}$ allele revealed a reduced expression of Mecp2 by $50 \%$ (Samaco, Fryer et al. 2008). These Mecp $2^{\text {flox } / Y}$ mice manifested a milder spectrum of disabilities, such as learning and motor deficits, decreased anxiety, and breathing dysfunction (Samaco, Fryer et al. 2008).

Overall, there is accumulating evidence that the central nervous system is highly sensitive to the functional level of Mecp2: certain levels of Mecp2 are required for the neuronal system to maintain its inhibitory and excitatory balance (Ramocki and Zoghbi 2008). Consequently, any gain or loss of Mecp2 over a narrow threshold level can lead to postnatal neurological impairment (Chao and Zoghbi 2012). This precise regulation of $M E C P 2$ expression to maintain the balance has important impacts, for example in the evaluation of potential therapeutic strategies for correcting the expression level of MECP2 in patients with MECP2 dysfunctions. To study the pathological mechanism underlying the neurological impairment of gain-of-function of 
MECP2, we established a transgenic mouse model with only mild overexpression of Mecp2. We aimed to identify and characterize the earliest pathologies elicited due to gain-of-function properties of $M E C P 2$.

To evaluate Mecp2 dosage effects, a Mecp2 ${ }^{W T}$ EGFP transgenic (TG) mouse was generated in which total Mecp2 (endogenous plus TG) was only mildly overexpressed at $~ 1.5 f o l d$ WT level. The TG Mecp2 protein which showed a similar expression pattern to endogenous Mecp2 in the hippocampus, was biologically functional, as cross breeding of these mice with Mecp2 null-mutant mice rescued major phenotypes in the null mutant mice. To assess the effect of mild overexpression of Mecp2 on neurons, neuronal parameters of cultured neurons were studied. TG neurons showed a reduction in the number of tertiary branching, but increased spine density and altered calcium homeostasis.

To study the phenotypical consequences of mildly overexpressing Mecp2 at $~ 1.5$ fold WT level, we performed a detailed behavioral characterization during early postnatal period and adulthood of male TG and WT mice. First, TG and WT control mice underwent neonatal assessments which comprised (1) maturation measures targeting physical development, (2) neurodevelopmental measures targeting the development of neurological reflexes and (3) the development of neuron-motor-coordination. The TG mice did not differ from their WT littermates on cursory neonatal observation, suggesting that mild Mecp2 overexpression at $\sim 1.5$ fold WT level did not alter the neonatal development.

The effect of mild Mecp2 overexpression was then investigated during adulthood. Adult TG and WT mice were tested to assess their basic behavior (activity, anxiety, exploratory), sensory (olfactory, vision and hearing), motor and sensorimotor gating functions, and social behavior (aggression) and also depression-like behavior (anhedonia). Most behavioral domains tested were not altered in TG mice. However, we found that TG mice displayed increased aggression towards intruder mice during the resident-intruder paradigm test for territorial aggression. This confirmed our observation that TG mice were more inclined to bite when handled. We also repeatedly observed spontaneous seizures in the TG mice. Thus, to assess seizure susceptibility, we induced seizures by pentylenetetrazole (PTZ) administration. Indeed, we detected increased susceptibility to PTZ-induced seizures in 30-week old TG mice. Interestingly, on the neuronal level, treatment of TG neurons with PTZ also led to a 
marked increase in amplitude and frequency of calcium spikes. Spontaneous and elicited calcium spikes (indicators of spontaneous discharges of neurons) are usually considered as a correlate of epileptic seizures (DeLorenzo, Sun et al. 2007). In conclusion, the results of the second project suggest that epileptic seizure propensity is highly sensitive to the functional level of Mecp2 and it appears as the primary symptoms when Mecp2 is mildly overexpressed in mice.

\section{b. Original publication}

Bodda $\mathrm{C}^{*}$, Tantra $\mathbf{M}^{*}$, Mollajew R, Arunachalam JP, Laccone FA, Can K, Rosenberger A, Mironov SL, Ehrenreich H and Mannan AU (2013): Mild overexpression of Mecp2 in mice causes a higher susceptibility toward seizures. The American Journal of Pathology

${ }^{\star}$ Equal contribution

\section{Own contribution:}

I was responsible for the very detailed behavioral characterization of several cohorts of transgenic mice with mild Mecp2 overexpression and their wild-type littermates. This included adult male mice testing as well as neonatal testing. I investigated the domains of basic behavior, sensory and motor functions, and social behavior. Not only did I perform the experiments, I also analyzed and interpreted the behavioral data which I presented in the figures for the publication (see Table 1, figure 2, supplemental figure S1, S4 and supplemental table S1). Moreover, I was involved in the conception, design, drafting, revision and publication of the manuscript: under the supervision of Prof. Hannelore Ehrenreich and PD Dr. Ashraf Mannan, I wrote and cited appropriately the materials and methods and results (including the interpretations of the results for the discussion) sections of the behavioral experiments of the manuscript. I prepared the above mentioned figures and tables and wrote the corresponding figure legends. 


\section{Mild Overexpression of Mecp2 in Mice Causes a Higher Susceptibility toward Seizures}

Chiranjeevi Bodda, ${ }^{\star \dagger}$ Martesa Tantra, ${ }^{\dagger \ddagger}$ Rustam Mollajew, ${ }^{\dagger \S}$ Jayamuruga P. Arunachalam, ${ }^{\star}$ Franco A. Laccone, ${ }^{\circledR}$ Karolina Can, ${ }^{*}$ Albert Rosenberger, ${ }^{\|}$Sergej L. Mironov, ${ }^{\dagger \S}$ Hannelore Ehrenreich, ${ }^{\dagger \ddagger}$ and Ashraf U. Mannan ${ }^{* \dagger}$

From the Institute of Human Genetics, * the Department of Neuro- and Sensory Physiology, ${ }^{\S}$ and the Department of Genetic Epidemiology, ${ }^{\|}$Center for Informatics, Statistics and Epidemiology, Universitätsmedizin Göttingen, Göttingen, Germany; the Center for Nanoscale Microscopy and Molecular Physiology of the Brain, ${ }^{\dagger}$ Göttingen, Germany; Institute of Medical Genetics, ${ }^{\circledR}$ Medical School of Vienna, Vienna, Austria; and the Division of Clinical Neuroscience, ${ }^{\ddagger}$ Max Planck Institute of Experimental Medicine, Göttingen, Germany

\author{
Accepted for publication \\ March 14, 2013. \\ Address correspondence to \\ Ashraf U. Mannan, Ph.D., \\ Institute of Human Genetics, \\ University of Goettingen, \\ Heinrich-Dueker-Weg 12, \\ D-37073, Goettingen, \\ Germany. E-mail: amannan@ \\ gwdg.de.
}

\begin{abstract}
An intriguing finding about the gene encoding methyl-CpG binding protein 2 (MeCP2) is that the loss-offunction mutations cause Rett syndrome and duplication (gain-of-function) of MECP2 leads to another neurological disorder termed MECP2 duplication syndrome. To ensure proper neurodevelopment, a precise regulation of MeCP2 expression is critical, and any gain or loss of MeCP2 over a narrow threshold level may lead to postnatal neurological impairment. To evaluate MeCP2 dosage effects, we generated Mecp $2^{\text {WT_EGFP }}$ transgenic (TG) mouse in which MeCP2 (endogenous plus TG) is mildly overexpressed (approximately $1.5 \times)$. The TG MeCP2 ${ }^{\text {WT_EGFP }}$ fusion protein is functionally active, as cross breeding of these mice with Mecp2 knockout mice led to alleviation of major phenotypes in the null mutant mice, including premature lethality. To characterize the Mecp $2^{\text {WT_EGFP }}$ mouse model, we performed an extensive battery of behavioral tests, which revealed that these mice manifest increased aggressiveness and higher pentylenetetrazole (PTZ)-induced seizure propensity. Evaluation of neuronal parameters revealed a reduction in the number of tertiary branching sites and increased spine density in Mecp $2^{W T E E G F}$ transgenic (TG) neurons. Treatment of TG neurons with epileptogenic compound-PTZ led to a marked increase in amplitude and frequency of calcium spikes. Based on our ex vivo and in vivo data, we conclude that epileptic seizures are manifested as the first symptom when MeCP2 is mildly overexpressed in mice. (Am J Pathol 2013, 183: 195-210; http://dx.doi.org/10.1016/j.ajpath.2013.03.019)
\end{abstract}

The $M E C P 2$ gene encodes for methyl-CpG-binding protein 2 (MeCP2). MeCP2 binds to methylated CpG DNA sequences and modulates transcription of genes in the vicinity of the methylated sequence. ${ }^{1}$ The ability of MeCP2 to bind to the methylated $\mathrm{CpGs}$, and its predominant localization to the highly methylated heterochromatin regions of the nucleus, ${ }^{2}$ led researchers to propose that $\mathrm{MeCP} 2$ may act as a global transcriptional repressor. ${ }^{3}$ Subsequently, the majority of studies substantiated the role of $\mathrm{MeCP} 2$ in repression of transcription (reviewed in Chahrour and Zoghbi ${ }^{4}$ and Guy et $\mathrm{al}^{5}$ ). Furthermore, MeCP2 was shown to interact with Sin3A and HDAC (histone deacetylases). ${ }^{6}$ By recruiting $\mathrm{HDAC}$ at the methylation loci, MeCP2 further represses transcription at such sites. However, recent studies have also highlighted the role of MeCP2 in transcriptional activation. It was shown that MeCP2 associates with actively transcribed genes. ${ }^{6,7} \mathrm{MeCP} 2$ promotes activation of transcription by associating with the transcriptional activator CREB1 (cAMP responsive element binding protein 1) on an active but not repressed form of the same gene. ${ }^{6}$ A multifaceted role of MeCP2 was further corroborated by the discoveries of its involvement in RNA splicing and chromatin compactions. ${ }^{8,9}$

Mutations in MECP2 gene have been shown to cause a neurodevelopmental disorder known as Rett syndrome (RTT). ${ }^{10}$ Classically, RTT patients develop normally until the age of 6 to 18 months. After this normal phase of development, RTT patients fall into a stage of developmental stagnation, followed by rapid regression. The patients start

\footnotetext{
Supported by the Cluster of Excellence and Deutsche Forschungsgemeinschaft Research Center Nanoscale Microscopy and Molecular Physiology of the Brain (A.U.M., S.L.M., and H.E.).

C.B. and M.T. contributed equally to this work.
} 
showing symptoms, such as progressive loss of motor skills, stereotypic hand movements, breathing abnormalities, microcephaly, epileptic seizures, and social interaction impairments reminiscent of autism. ${ }^{11}$ Due to X-chromosome inactivation, females are mosaics for MECP2 mutations, which can have a mitigating effect, with phenotypes ranging from classic RTT to asymptomatic individuals. ${ }^{12}$ However, male patients carrying MECP2 mutations usually show a very severe phenotype. They typically suffer from neonatal encephalopathy and die in early childhood. ${ }^{13}$ Until now, more than 700 MECP2 mutations have been reported for RTT (https://portal.biobase-international.com/hgmd/pro/all. php, HGMD Professional 2012.4, last accessed December 14, 2012). All types of mutations have been reported, such as missense, nonsense, splice site, deletion, and insertion. This wide spectrum of $M E C P 2$ mutations suggests loss-of-function as the likely common pathomechanism for RTT.

In addition to these loss-of-function mutations in the $M E C P 2$ gene, which cause RTT, until now more than 100 cases of copy number gains of the $M E C P 2$ gene have been reported. ${ }^{14,15}$ In the majority of cases, duplication of $M E C P 2$ locus was observed, however, in several instances, triplication was also detected. ${ }^{16,17}$ The gain of $M E C P 2$ lead to a neurological disorder termed $M E C P 2$ duplication syndrome, which primarily affects males who display variable phenotypes. Common symptoms include infantile hypotonia, severe mental retardation, autism or autistic features, poor speech development, recurrent infections, progressive spasticity, ataxia, and epilepsy. ${ }^{14,15,18}$ MECP2 duplication is $100 \%$ penetrant in males. ${ }^{14}$ The triplication of the $M E C P 2$ locus causes a more severe phenotype. ${ }^{17,19}$ Most of the reported $M E C P 2$ duplications are inherited, however, de novo cases have also been reported. ${ }^{18,20,21}$

Previously, a TG mouse (MECP2-Tg1) was generated that overexpresses $\mathrm{MeCP} 2$ at twice the level of endogenous MeCP2. ${ }^{22}$ The phenotypes of these mice are reminiscent of the human MECP2 duplication syndrome. Until the age of 10 to 12 weeks, these TG mice appear normal. Afterward, they develop progressive neurological symptoms that include stereotyped and repetitive movements, motor dysfunction, epilepsy, spasticity, ataxia, hypoactivity, kyphosis, and premature death. ${ }^{22}$ Increasing the copy numbers of Mecp 2 in the TG mice, resulting in higher MeCP2 levels, causes even more severe phenotypes. ${ }^{22}$ Interestingly, in another study, evaluation of a Mecp $2^{\text {flox }}$ allele revealed that due to the modification of the $3^{\prime} \mathrm{UTR}$ of Mecp 2 by insertion of a neomycin cassette, the expression of MeCP2 was reduced by $50 \% .^{23}$ These $M e c p 2^{\text {flox/Y }}$ mice manifest a spectrum of disabilities, such as learning and motor deficits, decreased anxiety, and breathing dysfunction.

Overall, it is evident that the central nervous system is highly sensitive to the functional level of $\mathrm{MeCP} 2$ and any gain or loss of MeCP2 over a narrow threshold level can lead to postnatal neurological impairment. This precise regulation of $\mathrm{MeCP} 2$ expression to ensure proper neurodevelopment has important ramifications regarding devising potential therapeutic strategies for correcting the expression level of MeCP2 in RTT patients. To study the pathomechanism underlying the neurological impairment in MECP2 duplication syndrome, a mouse model with only mild overexpression of MeCP2 may help to identify and characterize the earliest pathologies elicited due to gain-of-function properties of $M E C P 2$. Therefore, we generated Mecp $2^{W T \_E G F P}$ TG mice, which mildly overexpress MeCP2 (approximately $1.5 \times$ ).

\section{Materials and Methods}

\section{Generation of Mecp2 ${ }^{\text {WT_EGFP }}$ Transgenic Mouse Line}

A bacterial artificial chromosome (BAC) clone, plasmid (p)BAC_B22804 containing approximately $120 \mathrm{~Kb}$ of murine genomic fragment with intact Mecp2 gene and the flanking Opsinl and Irakl genes was used for generating the TG construct. $^{24}$ To generate pBAC_Mecp2 ${ }^{\text {WT_EGFP }}$ construct, the enhanced green fluorescent protein/kanamycin-resistant gene (EGFP/Kan) cassette was PCR-amplified using pEGFP1 vector as the template with primers containing $50 \mathrm{bp}$ flanking sequence from either side of the Mecp2 stop codon. Furthermore, the endogenous stop codon was replaced by two glycine residues in frame between the $\mathrm{MeCP} 2$ protein and the EGFP protein to facilitate the two proteins to fold and function independently. The amplified EGFP/Kan cassette was electroporated into Escherichia coli harboring the BAC clone and pGET recombination system ${ }^{25}$ to facilitate the homologous recombination of EGFP/Kan cassette at the site of stop codon of Mecp2. The correct insertion of EGFP/Kan cassette after the recombination event into the BAC clone was confirmed by sequencing. The Mecp 2 flanking genes, Opsinl and Irakl were deleted from the modified BAC clone using additional BAC recombineering with the Zeocin selection cassette (containing the BAC homology arms and Zeocin (InvivoGen, Toulouse, France) antibiotic marker gene driven by EM7 promoter from pSELECT vector) to avoid any additional phenotype arising from the overexpression of these genes. During the process of $O p \sin 1$ deletion, a $M l u$ I restriction site was introduced into the $\mathrm{BAC}$ clone.

The final BAC construct pBAC_Mecp2 $2^{\text {WT_EGFP }}$ was linearized with the $M l u$ I restriction enzyme and microinjected into the male pronuclei of the fertilized mouse oocytes derived from the FVB/N strain. Next, the injected oocytes were transplanted into the uteri of the foster mothers. The genomic DNA isolated from tail biopsies of newborn pups were analyzed for the presence of the transgene by PCR analysis, as previously described. ${ }^{24}$

\section{Western Blot Analysis}

For Western blot experiments, total protein extracts were isolated from brain biopsies of fresh male adult mice. In brief, the brain biopsies were collected in ice cold PBS and transferred to cold lysis buffer $(20 \mathrm{mmol} / \mathrm{L}$ PIPES [piperazine-N,N'-bis(2-ethanesulfonic acid)], $2 \mathrm{mmol} / \mathrm{L}$ 
EGTA, $1 \mathrm{mmol} / \mathrm{L}$ EDTA, $1 \mathrm{mmol} / \mathrm{L}$ DTT, and $0.3 \mathrm{mmol} / \mathrm{L}$ phenylmethylsulfonyl fluoride) containing protease inhibitors (Roche, Mannheim, Germany) followed by homogenization using tissue micromotor. The lysates were sonicated on ice and the supernatant was collected after centrifugation at $12,000 \mathrm{~g}$ for 10 minutes at $4^{\circ} \mathrm{C}$. An aliquot of $50 \mu \mathrm{g}$ protein lysates were denatured by boiling in a buffer containing SDS and dithiothreitol, which were resolved by SDS-PAGE using a NuPAGE 4 to $12 \%$ Bis-Tris precast gel in 2-(N-morpholino) ethanesulfonic acid buffer (Invitrogen, Karlsruhe, Germany). The resolved proteins were electrotransferred onto a nitrocellulose membrane (Amersham Biosciences, Braunschweig, Germany). Afterward, the membrane was blocked in 5\% lyophilized milk powder in Tris-buffered saline-Tween (100 $\mathrm{mmol} / \mathrm{L}$ Tris-Cl, $\mathrm{pH} 7.5 ; 150 \mathrm{mmol} / \mathrm{L} \mathrm{NaCl} ; 0.1 \%$ Tween 20 ) and then incubated with either anti-MeCP2 antibody (dilution $1: 8000$ ) raised in rabbit using MeCP2-strep tag fusion protein as antigen (Eurogentec, Cologne, Germany) or anti-GFP antibody (GeneTex, Eching, Germany) (dilution 1:5000) at $4^{\circ} \mathrm{C}$ overnight. The following day after washing, the immunoblot was incubated with secondary antibody conjugated with alkaline phosphatase (dilution 1: 10,000) (Sigma-Aldrich, Munich, Germany) for 1 hour at room temperature. Finally, Western blot analysis was developed by using nitroblue tetrazolium, 5bromo-4-chloro-3-indolyl phosphate (NBT/BCIP; Carl-Roth, Karlsruhe, Germany). The intensity of the protein bands in the Western blot analysis were quantified using ImageJ software version 1.46 (NIH, Bethesda, MD).

\section{RNA Preparation and RT-qPCR Analysis}

Total RNA was extracted from the cerebellum and hippocampus of 6-week-old male mice using TRIzol reagent (Invitrogen, Karlsruhe, Germany) according to the manufacturer's instructions. The cDNA was synthesized from $2 \mu \mathrm{g}$ of RNA using the SuperScript II (Invitrogen, Karlsruhe, Germany) and oligo-dT primer in a total volume of $20 \mu \mathrm{L}$. For real-time quantitative RT-PCR (RT-qPCR) analysis, the cDNA was further diluted (1:8) and was used as a template in Platinum SYBR Green qPCR SuperMix-UDG with ROX system (Invitrogen, Karlsruhe, Germany) with Mecp2 gene specific primers (Mecp2_F; 5'-TGGTAGCTGGGATGTTAGGG-3' and Mecp2_R; 5'-CCTTCTTGTCTTTCTTCGCC-3'). The RT-qPCR reactions were run in 7900 Sequence Detection System (Applied Biosystems, Darmstadt, Germany). The housekeeping gene, $\beta$-actin amplicon was used for data normalization. The RT-qPCR experiments were repeated four times with three technical replicate. The data were analyzed by SDS software version 2.2 (Applied Biosystems, Darmstadt, Germany) using standard curve method and Microsoft Excel version 2010 (Immeuble Laccolith, Luxembourg).

\section{Immunohistochemistry}

The adult male mice were transcardially perfused with $4 \%$ paraformaldehyde in PBS for 3 hours at $4^{\circ} \mathrm{C}$. The whole brain was dissected and submerged in $15 \%$ sucrose for 1 hour at $4{ }^{\circ} \mathrm{C}$ followed by incubation in $30 \%$ sucrose solution for 3 days, and then cryosectioned to $40 \mu \mathrm{m}$ thickness. The brain sections were washed three times with PBS for 5 minutes each and then permeabilized with $0.2 \%$ Triton-X for 30 minutes at $4^{\circ} \mathrm{C}$. Thereafter, the slides were washed again three times with PBS for 5 minutes and incubated in 4\% bovine serum albumin and $0.1 \%$ Tween-20 (Promega, Mannheim, Germany) for 1 hour at room temperature. The brain sections were then incubated with anti-MeCP2 antibody (1:200; Eurogentec, Cologne, Germany) at room temperature for 1 hour in $2 \%$ bovine serum albumin solution. After washing steps, the sections were incubated with secondary antibody conjugated with $\mathrm{Cy} 3$ (Sigma-Aldrich) for 1 hour at room temperature in 2\% bovine serum albumin solution. After final washing, the slides were air-dried and VectaShield solution (Linaris, Wertheim, Germany) was applied before mounting the slides with coverslips. The sections were observed under a BX60 fluorescence microscope (Olympus, Hamburg, Germany).

\section{Animal Management and Behavioral Analysis}

All experiments have been approved by the local Animal Care and Use Committee. Mice used for the experiments were kept in our behavioral unit under a temperaturecontrolled environment $\left(21 \pm 2^{\circ} \mathrm{C}\right)$ on a 12-hour light/dark cycle with food and water ad libitum, unless stated otherwise. A battery of behavioral tests were performed on preweaning and postweaning stages of mice. In all experiments, the experimenter (M.T.) was unaware (blinded) of the genotype.

\section{Drug Used in Animal Experiments}

Pentylenetetrazole (PTZ; Sigma-Aldrich Chemie GmbH, Taufkirchen, Germany), a noncompetitive GABA antagonist with epileptogenic properties was dissolved in saline for subcutaneous injection.

\section{Preweaning (Neonatal) Assessments}

Male TG mice were bred with FVB/N wild-type (WT) female mice to obtain WT and Mecp2 TG animals. A daily inspection for the presence of new litters in the cages was carried out twice a day, and the day a litter was first observed was scored as day 0 for that litter. After birth, animals were kept untouched in the home cage with their mothers until postnatal day (P) 3, and at P3 animals feet were tagged. Neurodevelopmental evaluation tests were performed daily starting from $\mathrm{P} 4$ until P21. The battery of tests provides an assessment of development throughout the neonatal period and the behaviors measured are expressed at different periods throughout the first 21 days of life. These tests have been extensively described elsewhere ${ }^{26-28}$ and consist of observations covering the maturation of physical landmarks, as well as developmental milestones (Supplemental Figure S1). Weaning was performed at 22 days of age. For the assessment of preweaning behavior, 14 male WT and 18 male TG litters were used. 


\section{Postweaning (Adult) Behavior}

Breeding pairs of Mecp 2 TG male and WT female FVB/N mice were used to obtain WT and Mecp2 TG mice. Shortly after weaning, mice were transferred to the behavioral unit for testing. On arrival and during the whole period of behavioral testing, mice were housed individually in standard plastic cages $(26.5 \times 20 \times 14 \mathrm{~cm})$. Adult behavioral testing was performed starting at the age of 5 weeks. The animal numbers of individual experiments are given in the legend of each corresponding figure.

\section{Experimental Design}

\section{Neonatal Assessments}

Neonatal assessments were comprised of three domains: i) maturation measures targeting physical development, ii) neurodevelopmental measures targeting the development of neurological reflexes, and iii) the development of neuromotor coordination.

\section{Maturation Measures (Physical Development)}

Body weight. The body weight of each pup was registered daily, starting from P4 through P21.

Ear opening. The day when an opening in the ear was visualized, the mouse was registered.

Eyes opened. The day when the eyes were opened, the mouse was registered. An eye was considered open when any visible break in the membrane was noticed.

\section{Neurodevelopmental Measures}

Surface righting reflex. Animals were restrained on their back on a table and then released. The time needed for each pup to right itself was recorded and the performance was monitored for 3 consecutive days, starting from P4. Score $O$ was assigned when pup did not show surface righting reflex and score 1 when surface righting reflex was present.

Postural reflex. Starting on P7 onward, animals were put in a small box and shaken up and down, left and right. Animals were tested until an appropriate response (animals splaying their four feet) was observed and scored as 1 .

Cliff avoidance. Observed daily from P6 until each test pup, when placed on an edge with forepaws and nose just over the edge, showed retraction within 10 seconds. Then score 1 was assigned.

Negative geotaxis reflex. Observed daily from P7 onward, animals were put in a head-down position on a $30^{\circ}$ inclined surface. The response of each pup was observed for 30 seconds and registered as shown in Supplemental Figure S1.

\section{Neuromotor Coordination Measures}

Wire suspension. The animals were forced to grasp a 3 $\mathrm{mm}$ wire and hang from it on their forepaws. Mice were tested starting on P10 onward until all test pups were able to hold the wire for 30 seconds.

Swimming ability. Mice were tested on P8, P12, and P14. On each observation day, one at a time, test pups were placed in a tank of water $\left(30^{\circ} \mathrm{C}\right)$ for a period of 5 to 15 seconds and observed for three aspects of swimming: i) direction, ii) head position relative to water surface, and iii) use of limbs. Scoring for direction was: sank, 0 ; floated, 1 ; swam in circle or arch, 2; straight, 3. Scoring for angle: submerged, 0; nose at the surface, 1 ; nose and top head at or above the surface but ears still below the surface, 2; similar to the last except for waterline is now at mid-ear level, 3 ; nose and top of head and ears above the surface, 4. Scoring for limb usage: no paddling, 0; paddling with all four limbs, 1; paddling with hind limbs only and forelimbs held stationary, 2.

\section{Adult Behavior}

\section{Elevated Plus Maze}

The mouse was placed in the central platform, facing an open arm of the plus maze inside a room with light intensity set to 130 lx. Behavior was recorded by an overhead video camera and a computer equipped with Viewer software version 2 (Biobserve $\mathrm{GmbH}$, Sankt Augustin, Germany) to calculate the time each animal spends in open or closed arms. The proportion of time spent in open arms was used for estimation of open arm aversion (fear equivalent).

\section{Open Field}

Spontaneous activity in the open field was tested in a gray Perspex arena (120 cm in diameter, $25 \mathrm{~cm}$ high) (Max Planck Institute for Biophysical Chemistry, Göttingen, Germany) placed inside a room with light intensity set to $130 \mathrm{~lx}$. The mouse was positioned in the center and allowed to explore the open field for 7 minutes. The behavior was recorded by a computer-linked overhead video camera. Viewer software was used to calculate velocity, distance traveled, and time spent in central, intermediate, or peripheral zones of the open field.

\section{Hole Board}

The hole board test measures exploratory activity. The apparatus consisted of a transparent Perspex chamber $(21 \times$ $21 \times 36 \mathrm{~cm}$ ) with a nontransparent floor raised $5 \mathrm{~cm}$ above the bottom of the chamber with 16 equally spaced holes $(2$ $\mathrm{cm}$ in diameter). Mice were allowed to explore the chamber for 5 minutes and the number of explored holes (head dips) was monitored by 2 layers of infrared photo beams connected to a computer with ActiMot software version 2 (TSE Systems GmbH, Bad Homburg, Germany). The light intensity was set to $5 \mathrm{~lx}$ during the test phase.

\section{Pre-Pulse Inhibition Test}

In this test of sensorimotor gating, individual mice were placed in small metal cages $(90 \times 40 \times 40 \mathrm{~mm})$ to restrict major movements and exploratory behavior. The cages were equipped with a movable platform floor attached to a sensor that records vertical movements of the floor. The cages were placed in four sound-attenuating isolation cabinets (TSE Systems $\mathrm{GmbH}$ ). Startle reflexes were evoked by acoustic stimuli delivered from a loudspeaker that was suspended above the 
cage and connected to an acoustic generator. The startle reaction to an acoustic stimulus, which evokes a movement of the platform and a transient force resulting from this movement of the platform, was recorded with a computer during a recording window of $260 \mathrm{msec}$ (beginning with the onset of prepulse) and stored for further evaluation. The recording window was defined from the onset of the acoustic stimulus. An experimental session consisted of a 2-minute habituation to $65 \mathrm{~dB}$ background white noise (continuous throughout the session), followed by a baseline recording for 1 minute at background noise. After baseline recording, 6 pulse-alone trials using startle stimuli of $120 \mathrm{~dB}$ intensity and $40 \mathrm{msec}$ duration were applied to decrease influence of within-session habituation. These data were not included in the analysis of the prepulse inhibition. For tests of prepulse inhibition, the 120 $\mathrm{dB} / 40 \mathrm{msec}$ startle pulse was applied either alone or preceded by a prepulse stimulus of $70 \mathrm{~dB}, 75 \mathrm{~dB}$, or $80 \mathrm{~dB}$ intensity at 20 msec duration. An interval of $100 \mathrm{~ms}$ with background white noise was used between each prepulse and pulse stimulus. The trials were presented in a pseudorandom order with an interval ranging from 8 to 22 seconds. The amplitude of the startle response (expressed in arbitrary units) was defined as the difference between the maximum force detected during a recording window and the force measured immediately before the stimulus onset. Amplitudes were averaged for each individual animal, separately for both types of trials (ie, stimulus alone or stimulus preceded by a prepulse). Prepulse inhibition was calculated as the percentage of the startle response using the following formula:

Prepulse inhibition $\%=100-[$ (startle amplitude after pre-

pulse and pulse $) /($ startle amplitude after pulse only $) \times 100]$.

Rota-Rod

Rota-rod is a test for motor function, balance, and coordination, and comprises a rotating drum (Ugo Basile Srl, Comerio, Italy) that is accelerated from 4 to 40 revolutions per minute over the course of 5 minutes. Each mouse was placed individually on a drum and the latency of falling from the drum was recorded using a stopwatch. To assess motor learning, the rota-rod test was repeated 24 hours later.

\section{Grip Strength}

A grip strength meter (TSE Systems GmbH) was used to assess forelimb grip strength. Mice were lifted and held by their tail so that their forepaws could grasp a wire grid. The mice were then gently pulled backward by the tail with their posture parallel to the surface of the table until they released the grid. The peak force applied by the forelimbs of the mouse was recorded in pond. Each mouse performed the test three times, and the average of the three trials was used for statistical analysis.

\section{Assessment of Hearing}

Individual mice were placed in small metal cages $(90 \times 40 \times$ $40 \mathrm{~mm}$ ) to restrict major movements and exploratory behavior. The cages were equipped with a movable platform floor attached to a sensor that records vertical movements of the floor. The cages were placed in four sound-attenuating isolation cabinets (TSE Systems GmbH). Startle reflexes were evoked by acoustic stimuli delivered from a loudspeaker that was suspended above the cage and connected to an acoustic generator. The startle reaction to an acoustic stimulus (pulse), which evokes a movement of the platform and a transient force resulting from this movement of the platform, was recorded with a computer during a recording window of $100 \mathrm{msec}$ and stored for further evaluation. The recording window was defined from the onset of the acoustic stimulus. An experimental session consisted of a 2minute habituation to $65 \mathrm{~dB}$ background white noise (continuous throughout the session), followed by a baseline recording for 1 minute at background noise. After baseline recording, stimuli of different intensity and fixed $40 \mathrm{msec}$ duration were presented. Stimulus intensity was varied between $65 \mathrm{~dB}$ and $120 \mathrm{~dB}$, such that 19 intensities from this range were used with $3 \mathrm{~dB}$ step. Stimuli of the each intensity were presented 10 times in a pseudorandom order with an interval ranging from 8 to 22 seconds. The amplitude of the startle response (expressed in arbitrary units) was defined as the difference between the maximum force detected during a recording window and the force measured immediately before the stimulus onset. Amplitudes of responses for each stimulus intensity were averaged for individual animals. Mean values for each experimental group were plotted on the graph to provide the stimulusresponse curves.

\section{Visual Cliff Test}

This test was used to assess more profound visual deficiencies (eg, in contrast perception). ${ }^{29}$ The test apparatus consisted of an open-topped box $(70 \times 35 \mathrm{~cm}$ floor, $30 \mathrm{~cm}$ high). The walls of the box were made from white Perspex, and the base was made from clear Perspex. The box was positioned on the edge of a laboratory bench so that half of the base was placed on the bench (ground side), and the other half over the edge of the bench, suspended $1 \mathrm{~m}$ above the floor (air side). Mice were placed in the middle of the base at the edge of the cliff, and their activity was monitored for 5 minutes by a computer and the video-tracking system Viewer 2 (Biobserve $\mathrm{GmbH}$ ). The percentage of time each mouse spent on the ground and the air side of the box was calculated. The light intensity inside the room was set to $150 \mathrm{~lx}$.

\section{Olfaction Test}

In the first 3 days mice were habituated to the test cages $(29.5 \times 18.5 \times 13 \mathrm{~cm})$; mice were placed in the cages for 2 $\times 20$ minutes per day. On day 3, mice were deprived of food for 24 hours before testing, with water ad libitum and a chocolate cookie was placed within the home cage. From days 4 to 6 , mice were again placed into test cages with 
a chocolate cookie inside $(2 \times 20$ minutes $)$. After habituation was finished on each day, mice received food for 1 hour per day within their home cages. On day 7, the test took place, and individual mice were placed into test cages, in which a piece of a chocolate cookie was hidden under 1.5 $\mathrm{cm}$ standard bedding at the end of the cage. The mouse was positioned in the right corner at the opposite end of the cage, and the food-finding time (ie, the time from the moment the mouse was placed into the cage to the time it located the cookie and initiated burrowing) was recorded. As soon as the cookie was uncovered, the mouse was not allowed to consume it and was removed from the cage. To control for other sensory, motor, or motivational deficiencies that might influence the food-finding latency, the test was repeated as previously described with a piece of chocolate cookie clearly visibly placed on the bedding. A fresh cage and bedding was used for each trial, and all mice were subjected to identical testing procedures. Cut off time was set to 3 minutes.

\section{Sucrose Preference Test}

The evaluation of anhedonia-like behavior was assessed using the sucrose preference test, which was performed using a two-bottle procedure. During the sucrose preference test, mice have free access to both water and a sucrose solution. Animals were first habituated for 48 hours to consume water from the two small $(100 \mathrm{~mL})$ bottles. After habituation, mice were deprived of water and the sucrose preference was measured during the next 3 days. The first two days served as a habituation to sucrose solution. The results of day 3 were used for the evaluation of sucrose preference. Each day, single-housed mice were presented for 60 minutes with two bottles (one with tap water and one with $2 \%$ sucrose solution) in the home cage. Consumption of water or sucrose solution was measured by weighing the bottles before and after the session. Bottles were counterbalanced across the left and the right sides of the cage, and their position was alternated from test to test. Sucrose preference (percentage) was calculated as follows:

$$
\text { preference }=\left[\frac{\text { sucrose solution intake }(\mathrm{mL})}{\text { total fluid intake }(\mathrm{mL})}\right] \times 100
$$

\section{Resident Intruder}

Intermale aggression was studied in the resident-intruder paradigm. As standard opponent males, we used groupcaged males of the same age, also from the same FVB/N strain. A standard opponent was introduced into the home cage of the tested resident male and observation started when a tested resident male sniffed the opponent for the first time. The observation was stopped immediately after the first attack (an attack being defined as a bite) to prevent wounding, but lasted 5 minutes if no attack occurred. The latency of attack was recorded by a stop watch.

\section{PTZ-Induced Seizure}

The method used here has been previously described by Ferraro et al. ${ }^{30}$ Seizure activity was induced in wakeful mouse using a single subcutaneous injection of $50 \mathrm{mg} / \mathrm{kg}$ body weight PTZ. After injection of the compound, the mouse was placed in a small, clear home cage and was closely observed for 30 minutes. Latencies to focal [partial clonic (PC)], generalized [generalized clonic (GC)], and maximal [tonic-clonic (TC)] behavioral seizures were recorded. Furthermore, four phases in the continuum of behavioral response to subcutaneous PTZ injection were defined as follows: i) hypoactivity (a progressive decrease in motor activity until the animal came to rest in a crouched or prone position with the abdomen in full contact with cage bottom); ii) partial clonus (PC) (clonus seizure activity affecting face, head, and/or forelimb or forelimbs); iii) generalized clonus (GC) (sudden loss of upright posture, whole body clonus involving all four limbs and tail, rearing and autonomic signs); and iv) tonicclonic (TC) (maximal) seizure (generalized seizure characterized by tonic hind-limb extension, also associated with death).

Finally, latencies to PC, GC, and TC seizures were summed to assign each mouse a seizure score that was used as a quantitative trait measure for mapping according to the following equation:

$$
\begin{aligned}
\text { Seizure score }= & {\left[(0.2)\left(\frac{1}{\text { PClatency }}\right)+(0.3)\left(\frac{1}{\text { GClatency }}\right)+\right.} \\
& \left.(0.5)\left(\frac{1}{\text { TClatency }}\right)\right] \times 1000
\end{aligned}
$$

The weighing factors $(0.2,0.3$, and 0.5$)$ in the equation were included as means of incorporating a measure of the progressive nature of the PTZ-induced seizure phenotype into the severity rating because GC is regarded as a more significant event than PC, and TC (hind-limb extension) is regarded as the most severe component of the phenotype. Therefore, the seizure score reflects the degree of progression of the seizure phenotype in each mouse. ${ }^{30}$

\section{Hind Limb Clasping Test}

To quantify hind-limb clasping, ${ }^{31}$ P40 stage mice were picked up from the cage by tail and suspended $10 \mathrm{~cm}$ above the cage for 30 seconds to allow the clasping of the hind limbs. During this time mice were videotaped. The video was analyzed, and the duration of hind-limb clasping was calculated for each mouse.

\section{Primary Neuronal Culture and Immunocytochemical Analysis}

For neuronal cell preparation, brains from three P7 stage male mice were collected and put into dissection solution (0.72 $\mathrm{g}$ bovine albumin, $0.347 \mathrm{~g} \mathrm{MgSO}_{4}$ in $240 \mathrm{~mL}$ Hanks 
medium) on ice. The hippocampus was dissected from the whole brain, the meninges were removed, and the tissue samples were cut into small pieces and incubated in Hanks solution for 5 to 10 minutes on ice.

The tissue samples were then digested at $37^{\circ} \mathrm{C}$ for 10 minutes with $6 \mathrm{mg}$ of trypsin and $60 \mu \mathrm{g}$ of DNAase (2000 $\mathrm{U} / \mu \mathrm{L})$ in $2 \mathrm{~mL}$ of digestion solution $(0.8 \mathrm{~g} \mathrm{NaCl}, 35 \mathrm{mg}$ $\mathrm{NaHCO}_{3}, 37 \mathrm{mg} \mathrm{KCl}, 99 \mathrm{mg} \mathrm{Na} \mathrm{HPO}_{4}$ in $100 \mathrm{~mL}$ of $\mathrm{H}_{2} \mathrm{O}$, $\mathrm{pH}$ approximately 7.3 to 7.4 ). The reaction was stopped by addition of $2.4 \mathrm{mg}$ of trypsin inhibitor (Sigma-Aldrich) in 4 $\mathrm{mL}$ of dissection solution and incubated at room temperature for 10 minutes. The samples were then incubated in a solution of $0.5 \mathrm{~mL}$ of horse serum and $2.5 \mathrm{~mL}$ of dissection solution for 10 minutes at room temperature. The digested hippocampal cells were triturated with three different sizes of pipettes coated with Sigmacote (SigmaAldrich).

The homogenate was centrifuged at $100 \times \mathrm{g}$ at $4{ }^{\circ} \mathrm{C}$ for 10 minutes. The cells obtained were resuspended in $1 \mathrm{~mL}$ of culture medium A [0.5 g D-glucose, $0.2 \mathrm{~mL}(12.5 \mathrm{mg} / \mathrm{mL})$ insulin (Sigma-Aldrich), $1 \mathrm{~mL}$ (200 mmol/L) GLUTAMAX (Gibco, Paisley, UK), $50 \mu \mathrm{L}(10 \mathrm{mg} / \mathrm{mL})$, gentamicin, $10 \mathrm{mg}$ transferring, and $10 \mathrm{~mL}$ horse serum up to $100 \mathrm{~mL}$ with minimal essential medium Eagle (Sigma-Aldrich). The cell suspension was seeded (4000 to 8000 cells per well) in preincubated culture plates with coverslips coated with poly-Dlysine and laminin (BD Bioscience, Heidelberg, Germany) in culture medium $\mathrm{A}$ and incubated at $37^{\circ} \mathrm{C}$ in $5 \% \mathrm{CO}_{2}$. The medium was replaced after overnight incubation. After 4 days, culture media was changed to medium B [50 $\mu \mathrm{L}$ gentamicin (Sigma-Aldrich), $125 \mu \mathrm{L}$ bFGF (Invitrogen, Karlsruhe, Germany), $250 \mu \mathrm{L}$ L-Glutamine, $2 \mathrm{~mL}$ B27 supplement (Gibco, Darmstadt, Germany), $125 \mu \mathrm{L}$ cytosine arabinoside (4 $\mathrm{mmol} / \mathrm{L}$ ) up to $100 \mathrm{~mL}$ with Neurobasal medium (Gibco, Darmstadt, Germany)]. The medium was changed every 2 days until the day of neuronal cell analysis, when cells were fixed and immunostained with anti-microtubule-associated protein 2 (MAP2) antibody (Cell Signaling, Frankfurt, Germany) (dilution 1: 500) (Abcam, Cambridge, UK) and visualized by the BX60 fluorescence microscope (Olympus, Hamburg, Germany).

The evaluation of neuronal parameters, such as cell soma area and number of primary, secondary, and tertiary branch sites, was performed by ImageJ software ${ }^{32}$ and the total neurite lengths were measured by NeuronJ software version $1.4 .2^{33}$ (http://www.imagescience.org/meijering/software/ neuronj, last accessed March 2012). The analysis was performed with the experimenter (C.B.) being unaware (or blinded) of the genotype. The average data were derived from WT and TG mice (9 to 10 each).

\section{Sholl Analysis}

To evaluate the complexity of a neuron, such as dendrite distribution and arborization, we performed Sholl analysis ${ }^{34}$ on captured images of primary neurons. In brief, concentric circles (Scholl lines) were drawn at $5 \mu \mathrm{m}$ increments up to a radius of $250 \mu \mathrm{m}$ from the centroid of the neuron soma until the tip of the longest neurite. The numbers of crossings made by neurites originating from soma through each Scholl line were calculated by ImageJ software. The crossings of neurites at each Scholl line were summed and the means and SD were calculated.

\section{Measurements of Calcium Influx in Primary Neurons}

Primary culture of hippocampal neurons was prepared as previously described. The coverslips with neurons were placed on the microscope stage (at room temperature) in a chamber continuously superfused at $34^{\circ} \mathrm{C}$ at $1 \mathrm{~mL}$ per minute with HEPES-buffered salt solution $(136 \mathrm{mmol} / \mathrm{L}$ $\mathrm{NaCl}, 5 \mathrm{mmol} / \mathrm{L} \mathrm{KCl}, 1.25 \mathrm{mmol} / \mathrm{L} \mathrm{CaCl}_{2}, 0.8 \mathrm{mmol} / \mathrm{L}$ $\mathrm{MgCl}_{2}, 6 \mathrm{mmol} / \mathrm{L}$ glucose, 10 HEPES, pH 7.4). The experimental solutions were produced by adding aliquots of corresponding stock solutions directly to the chamber solution. The neurons were treated with $1 \mu \mathrm{mol} / \mathrm{L}$ fura2-AM (calcium indicator dye, Invitrogen, Karlsruhe, Germany) for 20 minutes. The cells were viewed through a $40 \times$ objective in an upright Axioscope2 microscope (Zeiss, Goettingen, Germany). Excitation $(380 \mathrm{~nm})$ was generated by light-emitting diode and the fluorescence (520 $\pm 19 \mathrm{~nm}$ ) was recorded with a cooled CCD camera. Images were captured at intervals of 0.5 seconds, and analyzed using MetaMorph software (Roper Scientific, Planegg/ Martinsried, Germany). Free calcium values $(C)$ were obtained according to Grynkiewicz et $\mathrm{al}^{35}$ as:

$$
C=K \frac{1-f}{f-R}
$$

where the dissociation constant for calcium binding to fura-2 is $K=0.22 \mu \mathrm{mol} / \mathrm{L},{ }^{35} f=F / F_{\text {max }}$. The maximal fluorescence $F_{\max }$ was measured after application of 1 $\mu \mathrm{mol} / \mathrm{L}$ ionomycin in calcium-free solution and $F_{\min }$ was obtained after introduction of $5 \mathrm{mmol} / \mathrm{L}$ calcium into the chamber. The calibration gave $R=F_{\text {min }} / F_{\text {max }}=0.21 \pm$ 0.02 .

To examine slow calcium buffering in neurons, we induced calcium loads by depolarization-activated calcium influx with $50 \mathrm{mmol} / \mathrm{L} \mathrm{KCl}$ and followed calcium recovery to the basal levels. The time-constants of calcium recovery, together with depolarization-induced calcium increases and resting calcium levels allow to assess the contribution of mechanisms involved in calcium regulation. ${ }^{36}$ The mean values of time-constants, resting, and elevation of calcium were determined in the WT and TG cells. PTZ tests were applied to examine responses of neurons to epileptogenic stimuli. Calcium changes were recorded for 12 to 16 neurons in the image field. We determined the amplitude and frequency of calcium spikes, counted the number of active neurons, and averaged the data for six biological replicates of WT and $M e c p 2^{W T_{-} E G F P}$ TG neuron. 


\section{Golgi-Cox Staining}

The whole mouse brains were dissected from 3 weeks old mice and Golgi staining was performed using the FD Rapid GolgiStain Kit (FD Neurotechnologies, Columbia, MD). The dissected brains was rinsed with double distilled water and then immersed in a Solution A/B mixture (impregnation) for 6 to 7 days at room temperature. Afterward, the brains were incubated in Solution C (cryoprotection) for 2 days and thereafter frozen in anisopentane/dry ice bath. The brains were immediately sectioned into 100 to $150 \mu \mathrm{m}$ slices using a CM 1900 cryostat (Leica, Wetzlar, Germany) and the slices were mounted on slide pre-coated with $0.4 \%$ gelatin (Sigma-Aldrich). After the samples were dried, they were dehydrated by immersing them in solution with incremental proportion of ethanol. The staining procedure for development of the black precipitate was performed using kit reagents according to the manufacturer instruction. Afterward, coverslips were mounted using Eukitt quick hardening mounting medium (Fluka, Neu Ulm, Germany) and the slides were visualized using the BX60 microscope (Olympus, Hamburg, Germany).

The morphology of pyramidal neurons was traced from the captured images through a graph pad by using NeuronJ software and neuronal parameters were evaluated as described above. The spine density was calculated as average number of spines sprouting from a block unit of 10 $\mu \mathrm{m}$ of neurite. The average data were derived from WT and TG mice, 5 to 6 each.

\section{Statistical Analysis}

For neuronal parameters evaluation, the differences between WT and Mecp $2^{W T_{-} E G F P}$ transgenic neurons were calculated by applying Wilcoxon's Rank sum statistics or, if appropriate, by using the Student's $t$-test. We set the overall level of significance to $5 \%$. For the number of branching sites as the mainly focused phenotypes were corrected for multiple testing according to Bonferroni assuming four independent tests (area, length, number of spines per $10 \mu \mathrm{m}$, and branching points). Data are represented as means \pm SD in both the figures and text.

In the preweaning behavior assessment, regression analyses were used to compare the performance between TG and WT littermate control mice: variables scored 0 or 1 were analyzed by logistic regression. Additionally, Fischer's exact test for categorical data on each observation day and two-way repeated measures analysis of variance including Bonferroni post hoc test were used where applicable.

Statistical significance for adult behavioral phenotyping was evaluated using the $U$-test and two-way repeated measures analysis of variance including the Bonferroni post hoc test where applicable. A critical value for significance of $P<0.05$ was used throughout the study. Data are represented as means \pm SEM in both the figures and text.
For RT-qPCR data, the statistical significance between WT and Mecp $2^{W T \_E G F P}$ TG mice were calculated by Student's $t$-test.

All statistical analyses were performed with either GraphPad Prism 4.0 (La Jolla, CA) or SAS Version 9.2, SAS Institute Inc. (Cary, NC) or SPSS for Windows, version 17.0 (Chicago, IL).

\section{Results}

\section{Reducing Expression of MeCP2 from Mecp $2^{\text {WT_EGFP }}$ Allele}

The Mecp $2^{W T-E G F P}$ TG mouse was generated by microinjection of linearized pBAC_Mecp2 $2^{\mathrm{WT}}$ _EGFP construct (Figure 1A) into fertilized oocytes of FVB/N mice. For the generation of the pBAC_Mecp $2^{\text {WT_EGFP }}$ construct, we used a BAC clone (B22804). This BAC contains approximately $120 \mathrm{~kb}$ of a mouse genomic fragment, which consists of the complete Mecp2 gene including all fours exons, as well as flanking/regulatory sequences. ${ }^{24}$ The BAC clone also contains the complete transcriptional unit of Opsin1 gene, which is $5^{\prime}$ upstream of Mecp2 and part of the Irakl gene, which is located downstream $3^{\prime}$ end of the Mecp 2 gene. The sequences of Opsin1 and Irakl genes were deleted from the BAC clone by GET recombination, ${ }^{24}$ using the Zeocin selection cassette to exclude any toxic effect in the TG animals due to duplication of these genes (Figure 1A).

The pups obtained after microinjection were tested for the integration of the transgene into the genomic sequence by PCR genotyping using a primer pair specific for the transgene. ${ }^{24}$ Through PCR genotyping, several founders were identified, however, subsequent breeding and transmission analysis of Mecp $2^{W T \_E G F P}$ allele among the founders led to the establishment of one successful TG line. From the breeding of this TG line with $\mathrm{FVB} / \mathrm{N}$ mice, we obtained equal numbers of male and female mice suggesting that the transgene was integrated into an autosomal chromosome. The Mecp $2^{W T_{-} E G F P}$ TG mouse line was maintained in a heterozygote state $\left(\mathrm{T}^{\mathrm{WT} /-}\right)$ by backcrossing with FVB/N mice. From such breeding, we obtained 50\% TG mice $\left(\mathrm{T}^{\mathrm{WT} /}{ }^{-}\right)$and $50 \% \mathrm{WT}$ littermates.

To determine whether the MeCP2 ${ }^{\text {WT_EGFP }}$ is expressed in the TG mice, we performed Western blot analysis with total protein extracted from the brain biopsies from the adult Mecp $2^{W T-E G F P}$ TG male mice. The immunoblot with antibody against $\mathrm{MeCP} 2$ revealed expression of two $\mathrm{MeCP} 2$ proteins. In addition to the endogenous MeCP2 (approximately $70 \mathrm{kDa}$ ) band, a TG MeCP2 $2^{\mathrm{WT} \text {-EGFP }}$ band of approximately $100 \mathrm{kDa}$ in size was detected in the brain of TG mice. In contrast, only endogenous MeCP2 was detectable in WT mice (Figure 1B). Moreover, immunoblot analysis with green fluorescent protein antibody detected the approximately $100 \mathrm{kDa}$ MeCP2 ${ }^{\text {WT_EGFP }}$ protein, only in TG mice (Figure 1B). From Western blot analysis, it is obvious that the intensity of MeCP2 ${ }^{\mathrm{WT}}$ _EGFP 

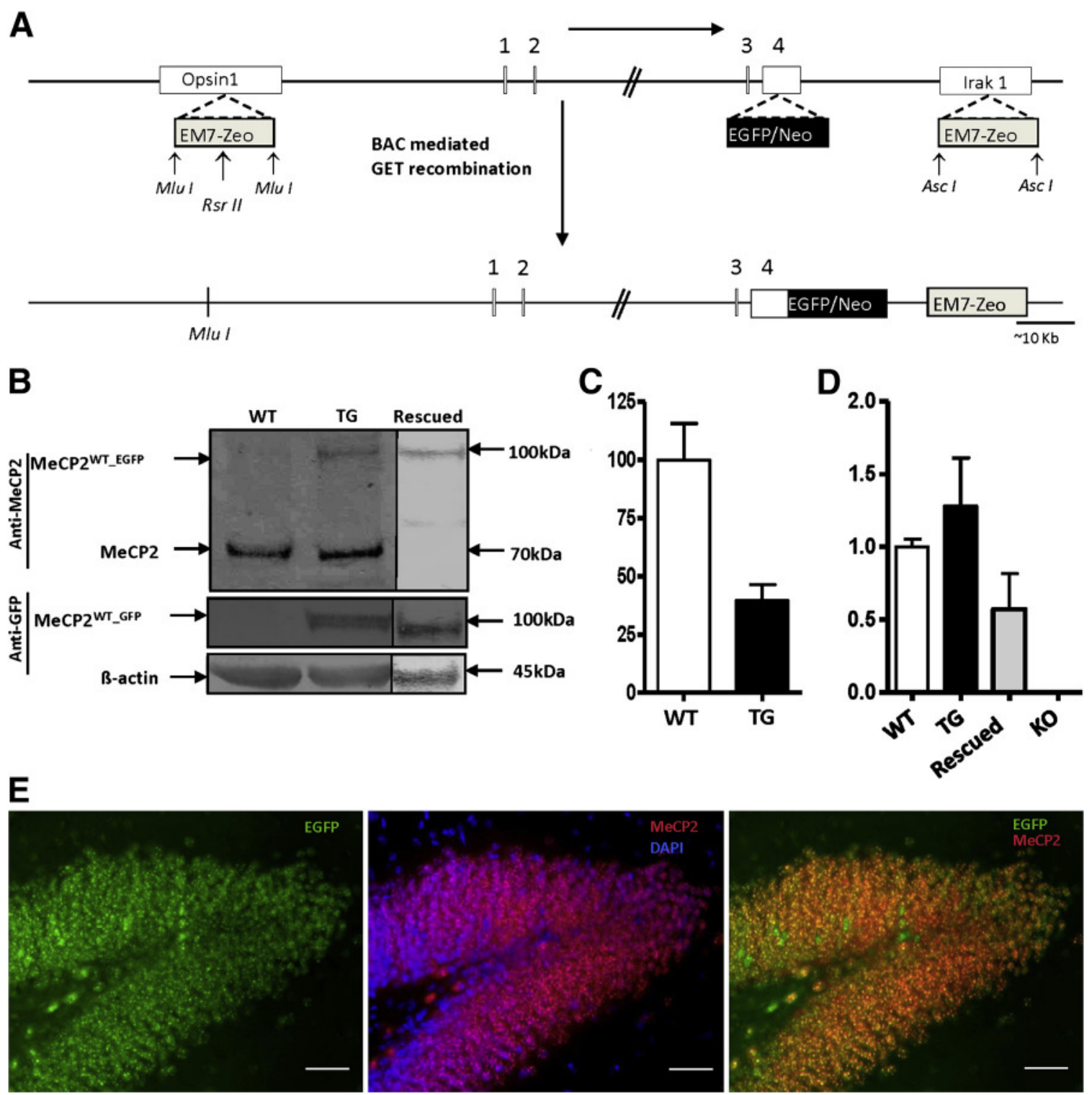

Figure 1 Expression analysis of MeCP2 from the BAC transgene. A: Schematic diagram illustrating the generation of the pBAC_Mecp2 ${ }^{\mathrm{WT}}-\mathrm{EGFP}$ construct using GET recombination in the BAC clone. An EGFP/Kan cassette was inserted in frame at stop codon of Mecp2 in exon 4 and was replaced with two glycine residues in the BAC clone. The sequences of Opsin1 and Irak1 genes were deleted from the BAC clone by GET recombination using the Zeocin selection cassette. B: Western blot analysis using MeCP2 antibody on the total protein extract isolated from brain samples revealed in addition to the endogenous MeCP2 $(70 \mathrm{kDa})$ an additional MeCP2 protein corresponding to MeCP2 ${ }^{\text {WT_EGFP }}$ of size approximately $100 \mathrm{kDa}$ in the TG adult mice.

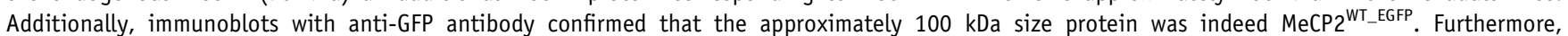
Western blot analysis with brain extract of rescued mice ( $\left.T^{\mathrm{WT}} /-:-/ \mathrm{y}\right)$, generated by breeding Mecp $2^{W T}$ EGFP TG mice with Mecp2 knockout mice revealed only expression of MeCP2 ${ }^{\text {WT_EGFP }}$ of size of approximately $100 \mathrm{kDa}$. C: Quantification of the protein bands intensities from at least five immunoblots revealed that relative expression of MeCP2 ${ }^{\text {WT_EGFP }}$ was approximately $40 \pm 4.1 \%$ to that of endogenous MeCP2. D: Quantitative real-time PCR analysis suggests that reduced expression of TG MeCP2 ${ }^{\text {WT_EGFP }}$ is due to the diminished level of mRNA. E: Immunohistochemical analysis of hippocampus showed a similar expression pattern for the TG MeCP2 ${ }^{\text {WT_EGFP }}$ protein as compared to endogenous MeCP2. K0, knockout mice adult mice. Scale bars: approximately $10 \mathrm{~kb}(\mathbf{A}) ; 50 \mu \mathrm{m}(\mathbf{E})$.

TG protein is remarkably lower as compared to endogenous $\mathrm{MeCP} 2$ band. This observation prompted us to quantify the relative intensity of the TG protein to endogenous protein by ImageJ software. Densitometrical quantification of the protein bands intensities from five independent immunoblots revealed that relative expression of MeCP2 $2^{\text {WT_EGFP }}$ was approximately $40 \pm 4.1 \%$ to that of endogenous MeCP2 (Figure 1C). To determine whether the reduced expression $\mathrm{MeCP} 2^{\text {WT_EGFP }}$ protein from the transgene was due to lower amount of mRNA, we performed quantitative real-time PCR analysis. Indeed, we also detected a reduced level of TG mRNA (Figure 1D).

To determine the cellular expression pattern of the $\mathrm{MeCP} 2^{\text {WT_EGFP }}$ protein, we performed immunohistochemical analysis of brain sections. MeCP2 ${ }^{\text {WT_EGFP }}$ protein was 
visualized by green fluorescent protein fluorescence and $\mathrm{MeCP} 2$ antibody staining, which also detected endogenous $\mathrm{MeCP} 2$ protein. The expression pattern of MeCP2 ${ }^{\mathrm{WT}}$ EGGF protein was similar to that of endogenous MeCP2 (Figure 1E and Supplemental Figure S2).

\section{Prolonging Lifespan of Mecp2 Null Mutant Mice on Expression of Mecp $2^{W T+E G F P}$ Allele}

We bred Mecp $2^{W T_{-} E G F P}$ TG mice with Mecp 2 knockout mice $^{31}$ to express the TG allele in Mecp2 null background $\left(\mathrm{T}^{\mathrm{WT} /}\right.$; $\left.;-/ \mathrm{y}\right)$. Western blot analysis with MeCP2 antibody using total protein extracted from the brain revealed expression of MeCP2 ${ }^{\text {WT_EGFP }}$ protein only (approximately $100 \mathrm{kDa}$ ) (Figure 1B). Remarkably, the premature lethality at the age of approximately 10 weeks, reported for null mutants, was rescued by the expression of the TG allele. These mice have a prolonged life span, as our observation over a period of approximately 9 months did not detect any early deaths. Hindlimb clasping, a typical feature of Mecp2 null mutant mice, was also absent in these mice (Supplemental Figure S3).

\section{Mecp2 ${ }^{\text {WT_EGFP }}$ Transgenic Mice Exhibit Increasing Aggressiveness and Seizure Propensity}

The TG mice appeared normal on cursory observation. To characterize the behavioral phenotype of Mecp $2^{W T_{-} E G F P} \mathrm{TG}$ mice, we performed a series of behavioral tests at both preweaning (0 to 3 weeks) and postweaning (after 5 weeks onward) stages.

\section{Pre-Weaning Behavior Analysis}

The number of animals used in the analysis of maturation markers and neurodevelopmental measures was: Mecp2 TG (TG) male $(n=18)$, WT littermate male $(n=14)$.

\section{Maturation Measures}

\section{Body Weight}

Body weight of animals was taken daily from P4 through P21 and the data were analyzed with two-way repeated measurement analysis of variance. We did not find any significant differences between genotypes in the body weight measured (Supplemental Table S1). As expected, all mice progressively increased their body weight as we observed from P4 through P21 (data not shown), indicating a comparable growth rate between TG and WT littermates.

\section{Ear and Eye Opening}

The day when at least one eye was open and the day an aperture was seen in the ear was registered daily starting from P4. No differences existed between genotypes regarding the day of the ear and the eye opening (Supplemental Table S1). All test mice have their ears and eyes fully opened on P14 (data not shown).
Neurodevelopmental Measures (Neurological Reflexes and Neuromotor Coordination)

There were no significant differences observed between groups in terms of the neurological reflexes tested in surface righting, postural, cliff avoidance, and negative geotaxis reflex (Supplemental Table S1). We also observed a comparable performance between groups during the acquisition of neuromotor coordination assessed from wire suspension and neonatal swimming activity (Supplemental Table S1).

\section{Postweaning (Adult) Behavior Analysis}

Adult behavioral phenotyping of male TG mice with mild Mecp 2 overexpression, as well as their WT littermates, was performed beginning at the age of 5 weeks (Table 1). Behavioral analysis was performed with less stressful tests at the beginning and more stressful tests toward the end in the following order: elevated plus maze, open field, hole board, prepulse inhibition, rota-rod, hearing, visual cliff, sucrose preference, grip strength, olfaction, resident intruder, and finally, PTZ-seizure induction. The number of animals used in the adult behavioral analysis is given in the corresponding figure legends of each read out. For behavioral analyses, we used 10 to $27 \mathrm{WT}$ and 6 to 22 TG mice (dependent on the complexity of the test, lower or higher numbers of mice are needed).

Table 1 Summary of Phenotypes in Adult Mecp2 $2^{\text {WT_EGFP }}$ Transgenic Mice

\begin{tabular}{|c|c|c|}
\hline Domain & Behavioral tests & WT versus TG \\
\hline \multicolumn{3}{|l|}{ Basic behavioral functions } \\
\hline Activity (locomotion) & Open filed & $P>0.05$ \\
\hline Anxiety & Elevated plus maze & $P>0.05$ \\
\hline Exploratory & Hole board & $P>0.05$ \\
\hline Anhedonia & Sucrose preference & $P>0.05$ \\
\hline $\begin{array}{l}\text { Seizure susceptibility } \\
\text { Motor functions }\end{array}$ & PTZ-induced seizure & $P<0.05$ \\
\hline $\begin{array}{l}\text { Motor learning and } \\
\text { coordination }\end{array}$ & Rota-rod & $P>0.05$ \\
\hline $\begin{array}{l}\text { Muscle strength } \\
\text { Sensory functions }\end{array}$ & Grip strength & $P>0.05$ \\
\hline Olfaction & Food finding & $P>0.05$ \\
\hline Vision & Visual cliff & $P>0.05$ \\
\hline $\begin{array}{l}\text { Hearing } \\
\text { Social behavior }\end{array}$ & Hearing (PPI set up) & $P>0.05$ \\
\hline $\begin{array}{l}\text { Aggression } \\
\text { Sensorimotor gating }\end{array}$ & Resident intruder & $P<0.05$ \\
\hline $\begin{array}{l}\text { Sensorimotor gating } \\
\quad \text { (information processing) }\end{array}$ & Prepulse inhibition & $P>0.05$ \\
\hline
\end{tabular}

Mild MeCP2 overexpression does not alter basic behavior, motor, sensory, or sensorimotor gating readouts in mice, but leads to alterations in social behavior (aggression) and seizure activity. Wild-type (WT) mice $=10$ to 27; transgenic (TG) mice $=6$ to 22. Data are presented as means \pm SEM. PPI, prepulse inhibition; PTZ, pentylenetetrazole. 


\section{Mild Overexpression of Mecp2 Selectively Influences} Behavioral Alterations

Although basic behavior, sensory, and motor functions, as well as sensorimotor gating remain unchanged by mild overexpression of Mecp2, our results revealed that social behavior and seizure propensity of TG animals overexpressing Mecp2 were altered (Table 1, Figure 2, and Supplemental Figure S4). During daily handling of the animals, we observed that mice displayed a higher tendency to bite when handled. We performed the resident-intruder paradigm to investigate whether mild Mecp 2 overexpression was associated with an increased aggressive behavior. Indeed, tested at the age of 8 weeks, we found that TG mice were more aggressive compared to the WT littermates (Figure 2A). The latency of the first attack of TG mice toward the intruder was significantly reduced, compared to their WT littermates $(P=0.048)$.

We also repeatedly observed spontaneous seizures in the TG mice. Thus, to assess seizure susceptibility, we induced seizures by administering PTZ $(50 \mathrm{mg} / \mathrm{kg}$ s.c. of body weight). ${ }^{30}$ We detected increased PTZ-induced seizure activity in 30-week-old TG mice $(P=0.042)$ compared to their WT littermates (Figure 2B).

\section{Mild Mecp2 Overexpression Causes Increase in Soma Size and Fewer Branching Sites in Cultured Neurons}

The ex vivo cultured hippocampal neurons are considered a well-characterized model for the evaluation of neuronal development. ${ }^{37}$ We cultured hippocampal neurons from P7 $M e c p 2^{W T-E G F P}$ TG and WT male mice to determine the effect of mild overexpression of Mecp2 during the process of neuronal development and differentiation. The primary neurons were cultured for 14 days, then immunostained with anti-MAP2 antibody (Figure 3, A and B). MAP2 is a neuron-specific cytoskeletal protein that is enriched in dendrites. ${ }^{38}$ The stained neurons were traced and analyzed
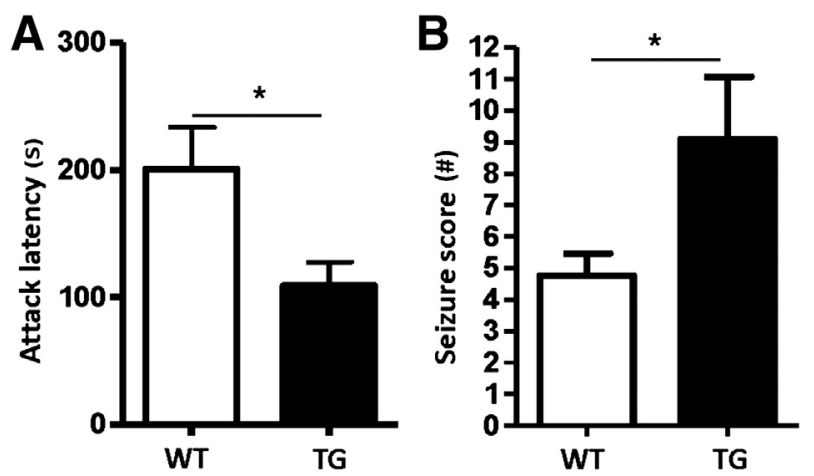

Figure 2 Phenotypic characterization of Mecp2 $2^{\text {WT_EGFP }}$ TG mice. A: To test aggressiveness, we performed the resident-intruder paradigm. The latency of the first attack of 8-week-old TG mice toward the intruder was significantly reduced, compared to their littermates, the WT mice. B: The 30-week-old TG mice also manifested increased sensitivity to PTZ-induced seizures. Mice (WT $=10$ to $27 ; \mathrm{TG}=6$ to 22). Data are presented as means \pm SEM. ${ }^{*} P<0.05$. by ImageJ software for calculating the soma size. The neuronal parameters, such as total length of neurites, number of neuritis, and number of primary, secondary, and tertiary branch sites, were evaluated by NeuronJ software.

The mean soma size of 91 TG neurons was slightly larger compared to those of 83 WT neurons $(P=0.0008)$ (Figure 3C). However, no significant difference could be seen for the total length of neurites projecting from TG neurons $(P$ $=0.1536$ ) (Figure 3D). Analysis of branching sites of neurites revealed an average lower number of branching sites in TG neurons, although this was clearly significant only for tertiary sites (primary sites: $P=0.6000$, secondary sites: $P=0.0496$, and tertiary sites: $P<0.0001$ ) (Figure $3, \mathrm{E}$ and G). To further evaluate the complexity of neurons, we performed Sholl analysis on the traced images. ${ }^{34}$ Sholl analysis is used to evaluate the morphological complexity of imaged neurons by counting the number of neurite intersections for concentric circles of increasing radius arising from the cell soma (Supplemental Figure S5). This quantitative analysis revealed diminished apical neurite branching in TG neurons.

\section{Pyramidal Neurons of Mecp $2^{\text {WT_EGFP }}$ Transgenic Mice Show Higher Spine Density}

To evaluate the effect of mild Mecp2 overexpression on neurons in vivo, we visualized the hippocampal neurons by Golgi-Cox staining of tissue sections. The Golgi-Cox staining allows high resolution visualization of dendrites and spines in the histological section, thus enabling us to calculate the qualitative and quantitative parameters of neurons. ${ }^{39-41}$ The stained pyramidal neurons (Figure 4A) were reconstructed by semiautomatic tracing using NeuronJ software (Figure 4B) and were analyzed similarly, as previously described for cultured neurons. We did not observe significant difference in soma size between TG and WT neurons (Figure 4C), although the total length of TG neurites was increased (Figure 4D). To determine the spine density of neurites, we used higher magnification images of basal dendrites from CA1 region (Figure 4E). Spine density for neurite unit length of $10 \mu \mathrm{m}$ revealed, on average, a slightly higher density of spines in TG neurites (mean, $14.4 \mu \mathrm{m}$ ) than WT neurites (mean, $13.6 \mu \mathrm{m} ; P=0.0324$ ) (Figure 4F).

\section{Calcium Homeostasis in Mecp $2^{\text {WT_EGFP }}$ Transgenic Neurons}

Calcium responses of cultured primary hippocampal neurons were evaluated by applying depolarization-induced calcium influx $(50 \mathrm{mmol} / \mathrm{L} \mathrm{KCl})$ and $\mathrm{IP}_{3}$-mediated calcium release from endoplasmic reticulum after activation of $\mathrm{P}_{2 \mathrm{Y}}$ receptors with $1 \mathrm{mmol} / \mathrm{L}$ ATP. ${ }^{42}$ Our measurements revealed that TG neurons in comparison to WT neurons, had higher levels of resting calcium $(0.075 \pm 0.05 \mu \mathrm{mol} / \mathrm{L}$ versus $0.052 \pm 0.03$ $\mu \mathrm{mol} / \mathrm{L}$ ), calcium elevations due to depolarization were also higher $(0.34 \pm 0.08 \mu \mathrm{mol} / \mathrm{L}$ versus $0.25 \pm 0.06 \mu \mathrm{mol} / \mathrm{L})$, and the amplitudes of ATP-driven calcium release were smaller 

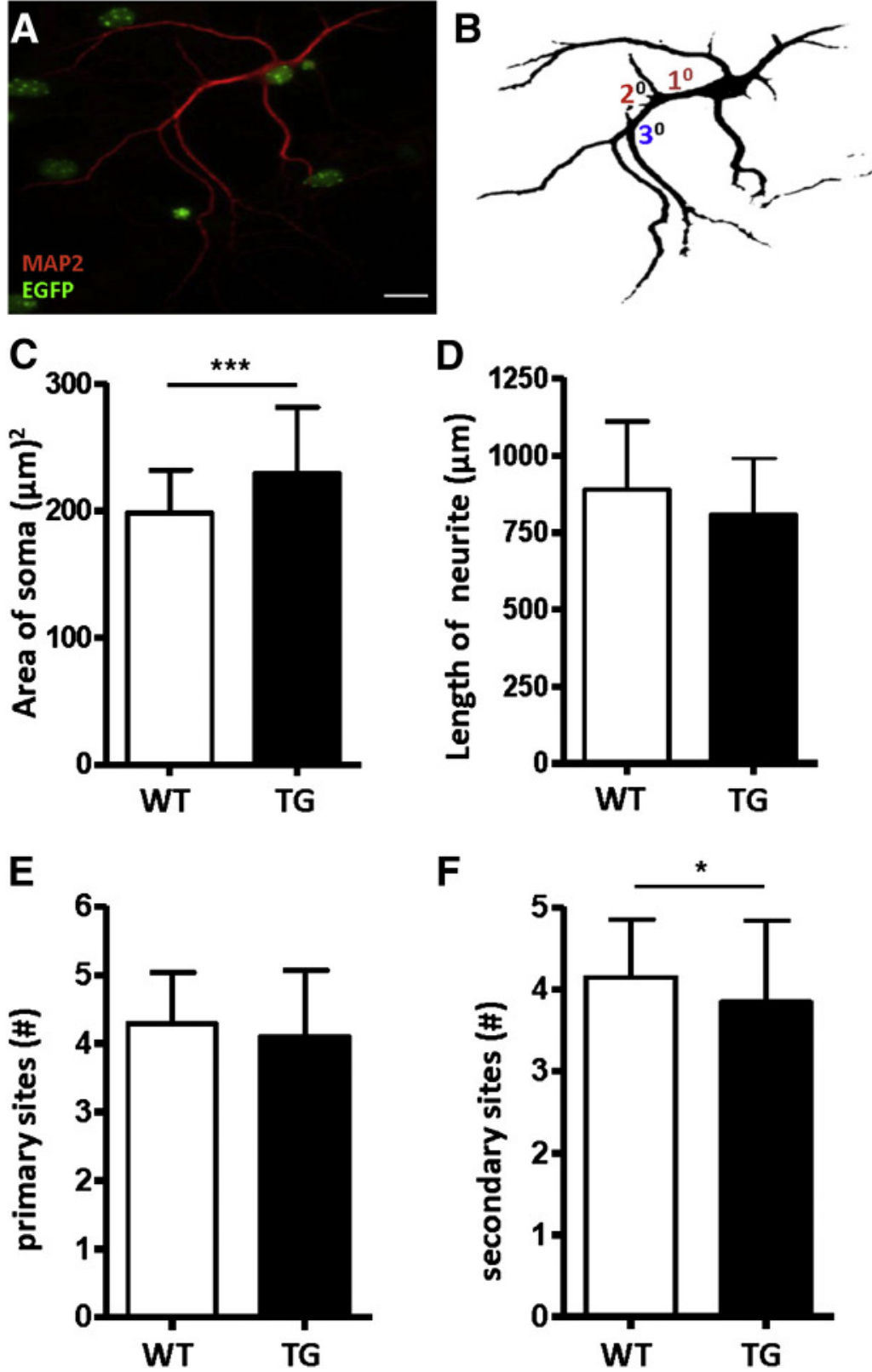

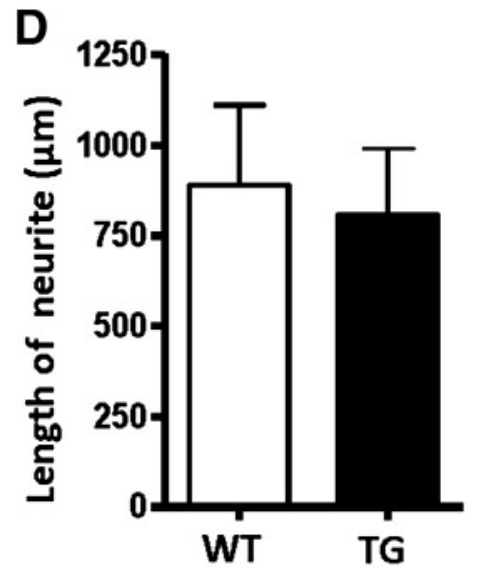

$\mathbf{F}$

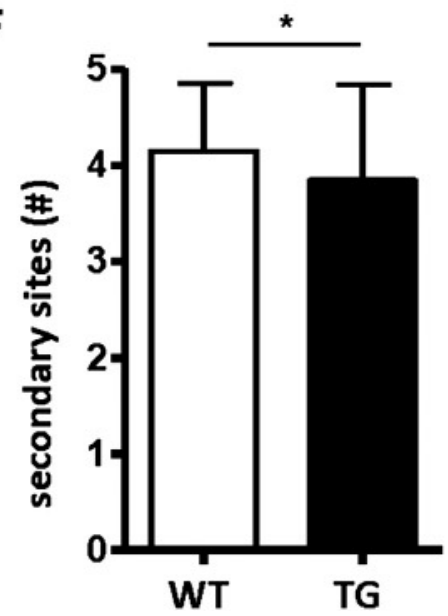

Figure 3 Evaluation of neuronal parameters of cultured (14 days) neurons from hippocampus of postnatal 7-day-old mice. A: Immunostaining of the TG Mecp $2^{\text {WT_EGFP }}$ primary neuronal cells with MAP2 (red), the MeCP2 ${ }^{\text {WT_EGFP }}$ expression was visualized by green fluorescence. B: The stained neuron images were traced by ImageJ and NeuronJ software and neuronal parameters were analyzed. C: The soma size of TG neurons was slightly larger compared to WT neurons. D: The total length of TG neurons was slightly smaller. $\mathbf{E}-\mathbf{G}$ : The numbers of branch sites were reduced in TG neurons, especially a significant reduction in tertiary branch sites was observed in TG neurons. Number of neurons (WT $=83 ;$ TG $=91$ ); $1^{0}$, primary; $2^{0}$, secondary; $3^{0}$, tertiary branch sites; Scale bar $=50 \mu \mathrm{m}(\mathbf{A})$. ${ }^{*} P<0.05,{ }^{* *} P<0.001$.

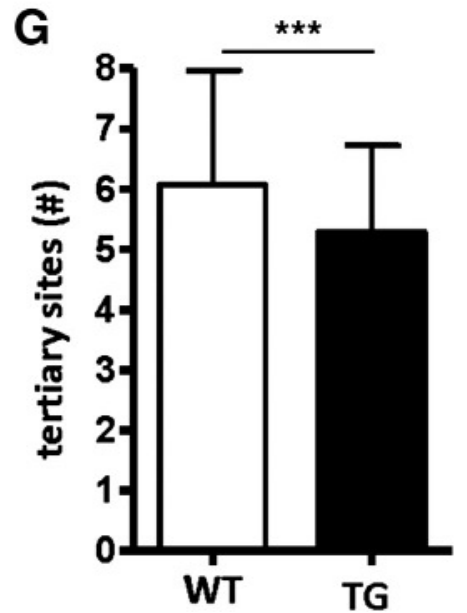

$(0.21 \pm 0.04 \mu \mathrm{mol} / \mathrm{L}$ versus $0.31 \pm 0.05 \mu \mathrm{mol} / \mathrm{L}$ ) (Figure $5, \mathrm{C}$ and D). The time course of calcium recovery in TG neurons was distinctly slower, both after depolarization and calcium release (Figure 5). The mean time constant of calcium recovery in WT was $6.1 \pm 0.4$ seconds (Figure 5A), and in TG cells it was approximately three times longer (18.6 \pm 1.2 seconds) (Figure 5B). We further observed that TG neurons were more prone to spontaneous calcium oscillations that may indicate intrinsic differences in the vulnerability toward external stimuli. Spontaneous calcium spiking was seldom observed in WT neurons (6 of 97 cells [6\%]), whereas in TG neurons, spontaneous activity was encountered more frequently (12 of 98 cells [12\%]).

In the behavioral test, the TG mice manifested a higher propensity toward PTZ-induced seizure activity (Figure 2C), we evaluated the response of cultured hippocampal neurons to PTZ. Spontaneous and elicited calcium spikes (indicators of spontaneous discharges of the neurons) are usually considered as a correlate of epileptic seizures. ${ }^{43,44}$ The WT neurons rarely showed spontaneous calcium transients and $10 \mathrm{mmol} / \mathrm{L}$ PTZ-induced only a weak effect (Figure 5E). The TG neurons frequently demonstrated spontaneous calcium spikes whose amplitude and frequency were markedly increased after PTZ treatment (Figure 5F).

\section{Discussion}

Evaluation of the quantitative effect of MeCP2 expression level on phenotypes is highly relevant, as duplication of 


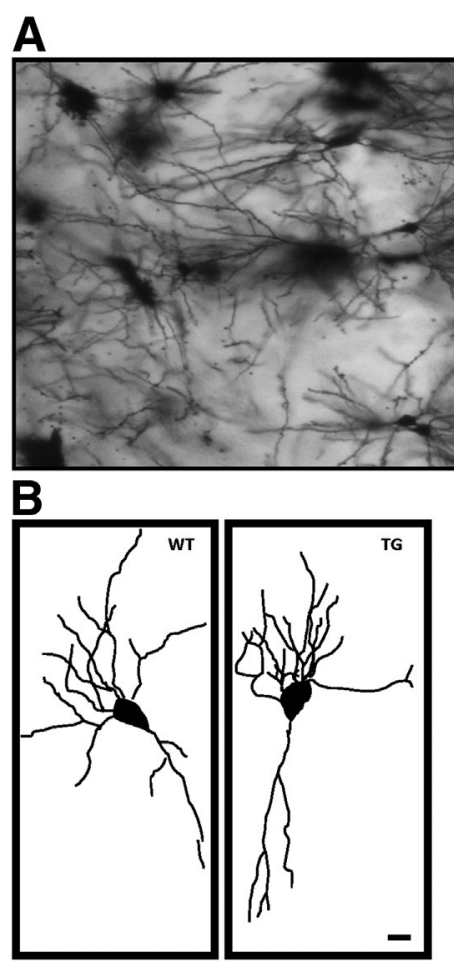

E

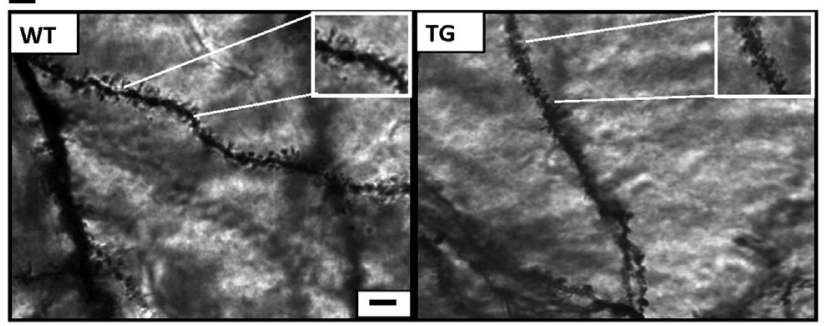

MECP2 causes severe neurological impairment, ${ }^{14,18,22}$ and on the contrary, loss of function mutations causes Rett syndrome. ${ }^{10}$ It is evident that precise regulation of $\mathrm{MeCP} 2$ expression is essential for proper neurodevelopment. To determine the consequence of $\mathrm{MeCP} 2$ dosage, we generated a TG Mecp $2^{W T-E G F P}$ mouse model mildly overexpressing $\mathrm{MeCP} 2$. The BAC construct used for the generation of $M e c p 2^{W T-E G F P}$ TG mice consist of complete promoter and regulatory elements necessary for the spatiotemporal expression pattern of MeCP2. ${ }^{22,24}$ In addition, to exclude the possibility of interference in the phenotype of Mecp $2^{W T-E G F P}$ mice due to overexpression of $O p \sin 1$ and $\operatorname{Irak1}$, we deleted both genes in the BAC construct. When expression pattern of MeCP2 ${ }^{\text {WT_EGFP }}$ from the transgene was compared with endogenous MeCP2, we observed a similar expression pattern, thus confirming that the BAC transgene mimics the endogenous MeCP2 expression. Additionally, in a previous study, we showed that expression pattern of a TG MeC$\mathrm{P}^{270}$ EGFP (generated from the same BAC clone) was comparable to the endogenous protein. ${ }^{24}$ In the pBAC Mecp2270_EGFP construct, the EGFP/Kan cassette was inserted after 270 amino acids into exon 4 of Mecp 2.
Figure 4 Characterization of pyramidal neurons in hippocampal sections by histological staining. A: The pyramidal neurons in the hippocampal sections of 3-week-old mice were visualized by Golgi-Cox staining. B: The neurons morphology reconstructed by semiautomatic tracing using NeuronJ software and neuronal parameters. $\mathrm{C}$ and D: The cell soma size were comparable between TG and WT neurons, total length of TG neurites was slightly longer. E: The spine density was calculated from higher magnification images. F: The spine density for neurite unit length of $10 \mu \mathrm{m}$ was higher in TG neurons. The spine density was plotted as mean value per unit length of $10 \mu \mathrm{m}$ for neurite length of approximately $2500 \mu \mathrm{m}$. Number of neurons (WT $=50 ; \mathrm{TG}=50)$. Scale bars: $100 \mu \mathrm{m}$ (A and B); $10 \mu \mathrm{m}(\mathbf{E}) .{ }^{*} P<0.05$.
$\mathbf{F}$

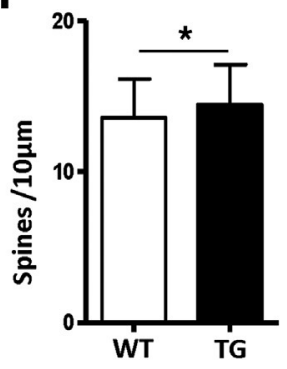

Overall, the MeCP2 270 EGFP $T G$ protein is equivalent to human p.R270X mutation. ${ }^{24}$

Interestingly, using Western blot analysis, the intensity of TG MeCP2 $2^{\text {WT_EGFP }}$ protein band was much weaker than endogenous MeCP2. Quantification of protein bands intensity revealed that the expression level of TG protein was nearly half $(40 \pm 4.1 \%)$ as compared to the endogenous $\mathrm{MeCP} 2$ protein. To determine whether the reduced expression from the transgene was due to lower level of mRNA or post-transcriptional regulation, we performed RT-qPCR analysis. Our data indicates that the diminished level of mRNA may be the underlying reason for lower level of TG MeCP2 $2^{\text {WT_EGFP }}$ protein. A previous study has shown that modification of $3^{\prime}$ UTR of Mecp 2 by insertion of a neomycin cassette and polyadenylation sequence in $M e c p 2^{\text {flox }}$ allele causes $50 \%$ reduction of expression as compared to the endogenous level in these mice. ${ }^{23}$ During the generation of Mecp $2^{W T_{-} E G F P}$ TG mice, we also modified the $3^{\prime}$ untranslated region of Mecp 2 by insertion of an EGFP/Kan cassette, which could be the underlying cause for reduced expression. However, a regulatory inhibition (at least partially) at the site of TG integration cannot be completely excluded. 


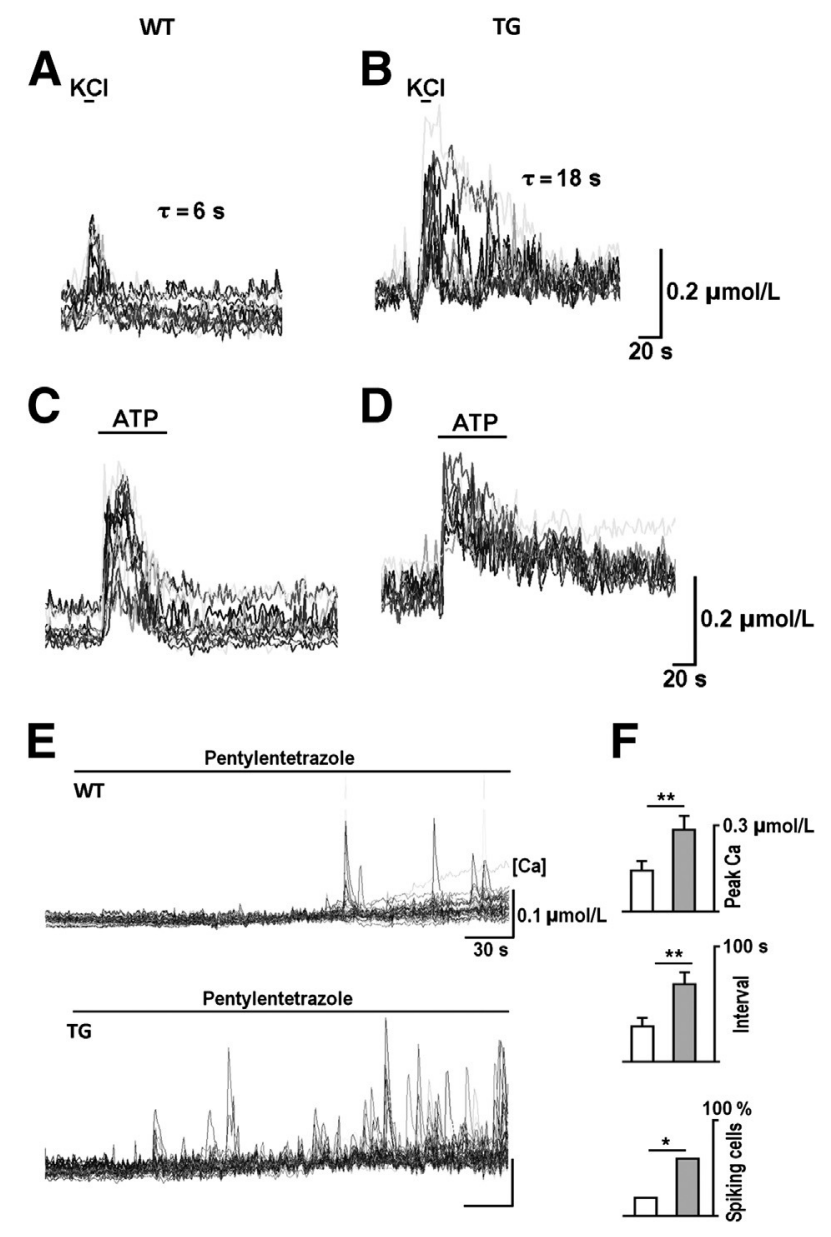

Figure 5 Impaired calcium response in hippocampal neurons, cultured for 14 days. A and B: Each panel shows calcium responses to membrane depolarization in 12 to 16 neurons. The depolarization with $50 \mathrm{mmol} / \mathrm{L} \mathrm{KCl}$ was followed by calcium recovery to basal levels. The mean time constant of calcium recovery was approximately three times longer in TG neurons than WT. $\mathbf{C}$ and $\mathbf{D}$ : Calcium release after activation of $P_{2 Y}$ receptors with $1 \mathrm{mmol} / \mathrm{L}$ ATP led to a smaller amount of calcium release in the TG neurons. E: Epileptogenic stimulation with $10 \mathrm{mmol} / \mathrm{L}$ PTZ-enhanced spontaneous calcium spikes substantially in the TG neurons. F: The amplitudes and frequencies of spontaneous calcium spikes and the number of active cells after PTZ exposure. The values were distinctly higher in TG neurons. White bars, WT; grey bars, TG. ${ }^{*} P<0.05,{ }^{*} P<0.01$.

To determine whether MeCP2 $2^{\text {WT_EGFP }}$ protein expressed from the TG locus is functionally active, we crossed Mecp $2^{W T_{-} E G F P}$ TG mice with Mecp 2 knockout mice. An expression level of approximately $50 \%$ of MeCP2 ${ }^{\text {WT_EGFP }}$ in the null background was able to rescue the premature lethality reported in these mice. We did not detect any indication of early death in these mice over a period of 9 months. In addition, the hind limb clasping phenotype of null mice was alleviated by transgene expression. Overall, these findings clearly indicate that MeCP2 ${ }^{\mathrm{WT}}$-EGFP protein is functional and C-termini fusion of EGFP protein does not interfere with biological function of $\mathrm{MeCP} 2$ in the transgene. Nevertheless, we wish to stress that approximately $50 \%$ of MeCP2 $2^{\text {WT_EGFP }}$ expression might not necessarily rescue all of the progressive phenotypes of null mutant mice and further investigation will be necessary to exclude any late stage symptoms.

Mecp $2^{W T_{-} E G F P}$ TG mice appear phenotypically normal and were negative for hind limb clasping (observation over a 9-month period). To identify any relevant behavioral symptoms in these mice, we performed a battery of tests involving basic and social behavior, motor performance, sensory functions, and sensorimotor gating. Our investigation revealed that in most behavioral parameters the performance of TG mice were comparable to that of WT siblings, and with the progression of age, there was no deterioration in the symptoms. However, the animals showed increased aggression and propensity toward PTZ-induced seizures. The phenotype of Mecp $2^{W T \_E G F P}$ TG mice (approximately $1.5 \times$ ) was in stark contrast to the previously reported $M E C P 2-\mathrm{Tg} 1$ mice $(2 \times)$ that manifested severe neurological phenotypes. ${ }^{22}$ $M E C P 2-\operatorname{Tg} 1$ mice display stereotyped and repetitive movements, motor dysfunction, epilepsy, spasticity, ataxia, hypoactivity, kyphosis, and premature death. ${ }^{22}$ In MECP2$\mathrm{Tg} 1$ mice, the phenotypes appear at an early stage (10 to 12 weeks) and deteriorate progressively. Overall, by means of our generated mouse model expressing approximately $1.5 \times$ the level of MeCP2, we could reinforce the notion that the effect of MeCP2 on neurodevelopment is quantitative. Furthermore, unlike doubling of $\mathrm{MeCP} 2$ dosage, which causes severe neurological impairment, only approximately $1.5 \times$ the level of overexpression of MeCP2 elicits a milder phenotype.

The expression level of MeCP2 is critical for early neuronal development and MeCP2 function is vital even at a single-neuron level. ${ }^{45}$ To assess the effect of mild overexpression (approximately $1.5 \times$ ) of $\mathrm{MeCP} 2$ on neurons, we evaluated neuronal parameters of cultured neurons. We observed a slight but statistically significant increase in soma size. The reduced number of tertiary branching sites in $M e c p 2^{W T_{-} E G F P} \mathrm{TG}$ as compared to WT neurons was also statistically significant. When we analyzed the neuronal parameters of Golgi-Cox stained, we observed no difference in soma size. This discrepancy could be due to sensitivity of two different experimental systems used for neuronal parameters. The morphological features of neurons are explicitly visible in primary culture; therefore, even subtle changes can be calculated. However, exogenous conditions in the primary culture can also create distortion, which might not be representative to an in vivo form. Remarkably, in Golgi-Cox stained neurons, we detected a slight increase (statistically significant) in spine density in the TG neurons. Previous studies have shown that loss of MeCP2 leads to substantial reduction in spine density, ${ }^{46-48}$ thus, it is reasonable to expect enhanced spine density during $\mathrm{MeCP} 2$ overexpression. Overall, it seems that mild overexpression of MeCP2 subtly deters the maturation of neurons.

Furthermore, we observed an impaired calcium homeostasis with a slower time course of calcium decay in $M e c p 2^{W T_{-} E G F P}$ TG neurons, which was manifested by an increase in basal calcium levels, prolongation of calcium 
recovery to basal levels after brief depolarization, and a smaller amount of calcium released from intracellular stores in TG neurons. The calcium imbalance could be the underlying cause for the observed exaggerated response of the TG neurons to epileptogenic compound-PTZ. ${ }^{30,44}$ Improper calcium handling can make these neurons more vulnerable to external stimuli encountered during early development. A similar disturbance in calcium homeostasis was also observed in Mecp 2 null mutant neurons, ${ }^{49}$ which suggest that both lack and overexpression of MeCP2 may lead to deleterious effect.

It is interesting to note that epileptic seizures are a major phenotype reported both in RTT and MECP2 duplication syndrome. Remarkably, MECP2 disruptions also lead to neuropsychiatric disorder, such as Angelman-like syndrome, and in many instances these patients also manifest epileptic seizures. ${ }^{50,51}$ In this context, it is noteworthy that the primary phenotype observed in the Mecp $2^{W T_{-} E G F P}$ TG mice was an increased propensity to epileptic seizures, as observed in our behavioral tests, as well as at the resolution of single neurons (ex vivo). In both in vivo and ex vivo settings, the seizure activity was induced by epileptogenic compound-PTZ, and in the TG condition, the induction was remarkably stronger.

In conclusion, it is evident from our study that epileptic seizure propensity is highly sensitive to the functional level of MeCP2 and it appears as a primary phenotype in the mouse due to mild overexpression of $\mathrm{MeCP} 2$. In future gene therapy approaches to correct the $\mathrm{MeCP} 2$ expression either in RTT or $M E C P 2$ duplication syndrome, the optimum dosage of the treatment may have to be determined so that epilepsy does not occur as a consequence.

\section{Acknowledgments}

We thank Heike Hühn for technical assistance; Stephan Wolf and Anja Ronnenberg for breeding, maintenance, and testing of mice; and Mayur Vadhvani for her help with neuronal parameters analysis.

\section{Supplemental Data}

Supplemental material for this article can be found at http://dx.doi.org/10.1016/j.ajpath.2013.03.019.

\section{References}

1. Lewis JD, Meehan RR, Henzel WJ, Maurer-Fogy I, Jeppesen P, Klein F, Bird A: Purification, sequence, and cellular localization of a novel chromosomal protein that binds to methylated DNA. Cell 1992, 69:905-914

2. Shahbazian MD, Antalffy B, Armstrong DL, Zoghbi HY: Insight into Rett syndrome: meCP2 levels display tissue- and cell-specific differences and correlate with neuronal maturation. Hum Mol Genet 2002, 11:115-124

3. Nan X, Campoy FJ, Bird A: MeCP2 is a transcriptional repressor with abundant binding sites in genomic chromatin. Cell 1997, 88:471-481
4. Chahrour M, Zoghbi HY: The story of Rett syndrome: from clinic to neurobiology. Neuron 2007, 56:422-437

5. Guy J, Cheval H, Selfridge J, Bird A: The role of MeCP2 in the brain. Annu Rev Cell Dev Biol 2011, 27:631-652

6. Chahrour M, Jung SY, Shaw C, Zhou X, Wong ST, Qin J, Zoghbi HY: MeCP2, a key contributor to neurological disease, activates and represses transcription. Science 2008, 320:1224-1229

7. Yasui DH, Peddada S, Bieda MC, Vallero RO, Hogart A, Nagarajan RP, Thatcher KN, Farnham PJ, Lasalle JM: Integrated epigenomic analyses of neuronal $\mathrm{MeCP} 2$ reveal a role for long-range interaction with active genes. Proc Natl Acad Sci USA 2007, 104: 19416-19421

8. Georgel PT, Horowitz-Scherer RA, Adkins N, Woodcock CL, Wade PA, Hansen JC: Chromatin compaction by human MeCP2. Assembly of novel secondary chromatin structures in the absence of DNA methylation. J Biol Chem 2003, 278:32181-32188

9. Young JI, Hong EP, Castle JC, Crespo-Barreto J, Bowman AB, Rose MF, Kang D, Richman R, Johnson JM, Berget S, Zoghbi HY: Regulation of RNA splicing by the methylation-dependent transcriptional repressor methyl-CpG binding protein 2. Proc Natl Acad Sci USA 2005, 102:17551-17558

10. Amir RE, Van den Veyver IB, Wan M, Tran CQ, Francke U, Zoghbi HY: Rett syndrome is caused by mutations in X-linked MECP2, encoding methyl-CpG-binding protein 2. Nat Genet 1999, 23:185-188

11. Neul JL, Kaufmann WE, Glaze DG, Christodoulou J, Clarke AJ, Bahi-Buisson N, Leonard H, Bailey ME, Schanen NC, Zappella M, Renieri A, Huppke P, Percy AK: Rett syndrome: revised diagnostic criteria and nomenclature. Ann Neurol 2010, 68:944-950

12. Wan M, Lee SS, Zhang X, Houwink-Manville I, Song HR, Amir RE, Budden S, Naidu S, Pereira JL, Lo IF, Zoghbi HY, Schanen NC, Francke U: Rett syndrome and beyond: recurrent spontaneous and familial MECP2 mutations at CpG hotspots. Am J Hum Genet 1999, 65:1520-1529

13. Kriaucionis $\mathrm{S}$, Bird A: DNA methylation and Rett syndrome. Hum Mol Genet 2003, 12 Spec No 2:R221-R227

14. Ramocki MB, Tavyev YJ, Peters SU: The MECP2 duplication syndrome. Am J Med Genet A 2010, 152A:1079-1088

15. Van Esch H: MECP2 Duplication Syndrome. Mol Syndromol 2012, 2:128-136

16. Carvalho CM, Ramocki MB, Pehlivan D, Franco LM, GonzagaJauregui C, Fang P, McCall A, Pivnick EK, Hines-Dowell S, Seaver LH, Friehling L, Lee S, Smith R, Del Gaudio D, Withers M, Liu P, Cheung SW, Belmont JW, Zoghbi HY, Hastings PJ, Lupski JR: Inverted genomic segments and complex triplication rearrangements are mediated by inverted repeats in the human genome. Nat Genet 2011, 43:1074-1081

17. Carvalho CM, Zhang F, Liu P, Patel A, Sahoo T, Bacino CA, Shaw C, Peacock S, Pursley A, Tavyev YJ, Ramocki MB, Nawara M, Obersztyn E, Vianna-Morgante AM, Stankiewicz P, Zoghbi HY, Cheung SW, Lupski JR: Complex rearrangements in patients with duplications of MECP2 can occur by fork stalling and template switching. Hum Mol Genet 2009, 18:2188-2203

18. Ramocki MB, Peters SU, Tavyev YJ, Zhang F, Carvalho CM, Schaaf CP, Richman R, Fang P, Glaze DG, Lupski JR, Zoghbi HY: Autism and other neuropsychiatric symptoms are prevalent in individuals with MeCP2 duplication syndrome. Ann Neurol 2009, 66: $771-782$

19. del Gaudio D, Fang P, Scaglia F, Ward PA, Craigen WJ, Glaze DG, Neul JL, Patel A, Lee JA, Irons M, Berry SA, Pursley AA, Grebe TA, Freedenberg D, Martin RA, Hsich GE, Khera JR, Friedman NR, Zoghbi HY, Eng CM, Lupski JR, Beaudet AL, Cheung SW, Roa BB: Increased MECP2 gene copy number as the result of genomic duplication in neurodevelopmentally delayed males. Genet Med 2006, 8:784-792

20. Clayton-Smith J, Walters S, Hobson E, Burkitt-Wright E, Smith R, Toutain A, Amiel J, Lyonnet S, Mansour S, Fitzpatrick D, Ciccone R, Ricca I, Zuffardi O, Donnai D: Xq28 duplication presenting with 
intestinal and bladder dysfunction and a distinctive facial appearance. Eur J Hum Genet 2009, 17:434-443

21. Smyk M, Obersztyn E, Nowakowska B, Nawara M, Cheung SW, Mazurczak T, Stankiewicz P, Bocian E: Different-sized duplications of Xq28, including MECP2, in three males with mental retardation, absent or delayed speech, and recurrent infections. Am J Med Genet B Neuropsychiatr Genet 2008, 147B:799-806

22. Collins AL, Levenson JM, Vilaythong AP, Richman R, Armstrong DL, Noebels JL, David Sweatt J, Zoghbi HY: Mild overexpression of $\mathrm{MeCP} 2$ causes a progressive neurological disorder in mice. Hum Mol Genet 2004, 13:2679-2689

23. Samaco RC, Fryer JD, Ren J, Fyffe S, Chao HT, Sun Y, Greer JJ, Zoghbi HY, Neul JL: A partial loss of function allele of methyl-CpGbinding protein 2 predicts a human neurodevelopmental syndrome. Hum Mol Genet 2008, 17:1718-1727

24. Kifayathullah LA, Arunachalam JP, Bodda C, Agbemenyah HY, Laccone FA, Mannan AU: MeCP2 mutant protein is expressed in astrocytes as well as in neurons and localizes in the nucleus. Cytogenet Genome Res 2010, 129:290-297

25. Orford M, Nefedov M, Vadolas J, Zaibak F, Williamson R, Ioannou PA: Engineering EGFP reporter constructs into a $200 \mathrm{~kb}$ human beta-globin BAC clone using GET recombination. Nucleic Acids Res 2000, 28:E84

26. Schapiro S, Salas M, Vukovich K: Hormonal effects on ontogeny of swimming ability in the rat: assessment of central nervous system development. Science 1970, 168:147-150

27. Vorhees CV, Butcher RE, Brunner RL, Sobotka TJ: A developmental test battery for neurobehavioral toxicity in rats: a preliminary analysis using monosodium glutamate calcium carrageenan, and hydroxyurea. Toxicol Appl Pharmacol 1979, 50:267-282

28. Santos M, Silva-Fernandes A, Oliveira P, Sousa N, Maciel P: Evidence for abnormal early development in a mouse model of Rett syndrome. Genes Brain Behav 2007, 6:277-286

29. Crawley JN: Behavioral phenotyping of transgenic and knockout mice: experimental design and evaluation of general health, sensory functions, motor abilities, and specific behavioral tests. Brain Res 1999, 835:18-26

30. Ferraro TN, Golden GT, Smith GG, St Jean P, Schork NJ, Mulholland N, Ballas C, Schill J, Buono RJ, Berrettini WH: Mapping loci for pentylenetetrazol-induced seizure susceptibility in mice. J Neurosci 1999, 19:6733-6739

31. Guy J, Hendrich B, Holmes M, Martin JE, Bird A: A mouse Mecp2null mutation causes neurological symptoms that mimic Rett syndrome. Nat Genet 2001, 27:322-326

32. Meijering E, Jacob M, Sarria JC, Steiner P, Hirling H, Unser M: Design and validation of a tool for neurite tracing and analysis in fluorescence microscopy images. Cytometry A 2004, 58:167-176

33. Abràmoff $\mathrm{MD}$, Magalhães $\mathrm{PJ}$, Ram SJ: Image Processing with ImageJ. Biophotonics International 2004, 11:36-42

34. Sholl DA: Dendritic organization in the neurons of the visual and motor cortices of the cat. J Anat 1953, 87:387-406

35. Grynkiewicz G, Poenie M, Tsien RY: A new generation of $\mathrm{Ca} 2+$ indicators with greatly improved fluorescence properties. J Biol Chem 1985, 260:3440-3450
36. Mironov SL: Plasmalemmal and intracellular $\mathrm{Ca} 2+$ pumps as main determinants of slow $\mathrm{Ca} 2+$ buffering in rat hippocampal neurones. Neuropharmacology 1995, 34:1123-1132

37. Dotti CG, Sullivan CA, Banker GA: The establishment of polarity by hippocampal neurons in culture. J Neurosci 1988, 8:1454-1468

38. Kosik KS, Finch EA: MAP2 and tau segregate into dendritic and axonal domains after the elaboration of morphologically distinct neurites: an immunocytochemical study of cultured rat cerebrum. J Neurosci 1987, 7:3142-3153

39. Graveland GA, Williams RS, DiFiglia M: Evidence for degenerative and regenerative changes in neostriatal spiny neurons in Huntington's disease. Science 1985, 227:770-773

40. Mendizabal-Zubiaga JL, Reblet C, Bueno-Lopez JL: The underside of the cerebral cortex: layer V/VI spiny inverted neurons. J Anat 2007, 211:223-236

41. Pilati N, Barker M, Panteleimonitis S, Donga R, Hamann M: A rapid method combining Golgi and Nissl staining to study neuronal morphology and cytoarchitecture. J Histochem Cytochem 2008, 56: 539-550

42. Mironov SL: Metabotropic ATP receptor in hippocampal and thalamic neurones: pharmacology and modulation of $\mathrm{Ca} 2+$ mobilizing mechanisms. Neuropharmacology 1994, 33:1-13

43. DeLorenzo RJ, Sun DA, Blair RE, Sombati S: An in vitro model of stroke-induced epilepsy: elucidation of the roles of glutamate and calcium in the induction and maintenance of stroke-induced epileptogenesis. Int Rev Neurobiol 2007, 81:59-84

44. Trevelyan AJ, Sussillo D, Watson BO, Yuste R: Modular propagation of epileptiform activity: evidence for an inhibitory veto in neocortex. J Neurosci 2006, 26:12447-12455

45. Chao HT, Zoghbi HY, Rosenmund C: MeCP2 controls excitatory synaptic strength by regulating glutamatergic synapse number. Neuron 2007, 56:58-65

46. Belichenko NP, Belichenko PV, Mobley WC: Evidence for both neuronal cell autonomous and nonautonomous effects of methylCpG-binding protein 2 in the cerebral cortex of female mice with Mecp2 mutation. Neurobiol Dis 2009, 34:71-77

47. Stuss DP, Boyd JD, Levin DB, Delaney KR: MeCP2 mutation results in compartment-specific reductions in dendritic branching and spine density in layer 5 motor cortical neurons of YFP-H mice. PLoS One 2012, 7:e31896

48. Landi S, Putignano E, Boggio EM, Giustetto M, Pizzorusso T, Ratto GM: The short-time structural plasticity of dendritic spines is altered in a model of Rett syndrome. Sci Rep 2011, 1:4548

49. Mironov SL, Skorova E, Hartelt N, Mironova LA, Hasan MT, Kugler S: Remodelling of the respiratory network in a mouse model of Rett syndrome depends on brain-derived neurotrophic factor regulated slow calcium buffering. J Physiol 2009, 587:2473-2485

50. Milani D, Pantaleoni C, D'Arrigo S, Selicorni A, Riva D: Another patient with MECP2 mutation without classic Rett syndrome phenotype. Pediatr Neurol 2005, 32:355-357

51. Watson P, Black G, Ramsden S, Barrow M, Super M, Kerr B, Clayton-Smith J: Angelman syndrome phenotype associated with mutations in MECP2, a gene encoding a methyl CpG binding protein. J Med Genet 2001, 38:224-228 


\section{Figure: S1}

\begin{tabular}{|c|c|c|c|c|c|}
\hline \multirow{2}{*}{ Domain } & \multicolumn{5}{|c|}{ Score } \\
\hline & 0 & 1 & 2 & 3 & 4 \\
\hline \multicolumn{6}{|l|}{ Physical development } \\
\hline Ear opening & Closed & Open & & & \\
\hline Eye opening & Closed & Open & & & \\
\hline \multicolumn{6}{|l|}{ Neurodevelopment } \\
\hline Surface righting reflex (age 4d) & $\begin{array}{l}\text { Stay in dorsal } \\
\text { position }\end{array}$ & Rights itself & & & \\
\hline Postural reflex (age 7d onwards) & Not present & Present & & & \\
\hline Cliff avoidance (age 6d onwards) & Fall down & Turns away from edg & & & \\
\hline $\begin{array}{l}\text { Negative geotaxis reflex } \\
\text { (age } 7 \mathrm{~d} \text { onwards) }\end{array}$ & Does not move & Turns and freezes & Turns and climbs & & \\
\hline Wire suspension (age 10d onwards) & Not present & Present & & & \\
\hline \multicolumn{6}{|l|}{ Swimming activity (age $8,12,14$ d) } \\
\hline \multirow{2}{*}{\multicolumn{6}{|c|}{$\begin{array}{l}\text { Paddling with hindlimbs, } \\
\text { forelimbs held stationary }\end{array}$}} \\
\hline & & & & & \\
\hline Direction & Drowning & Floating & In circle & Straight & \\
\hline
\end{tabular}


Figure: S2
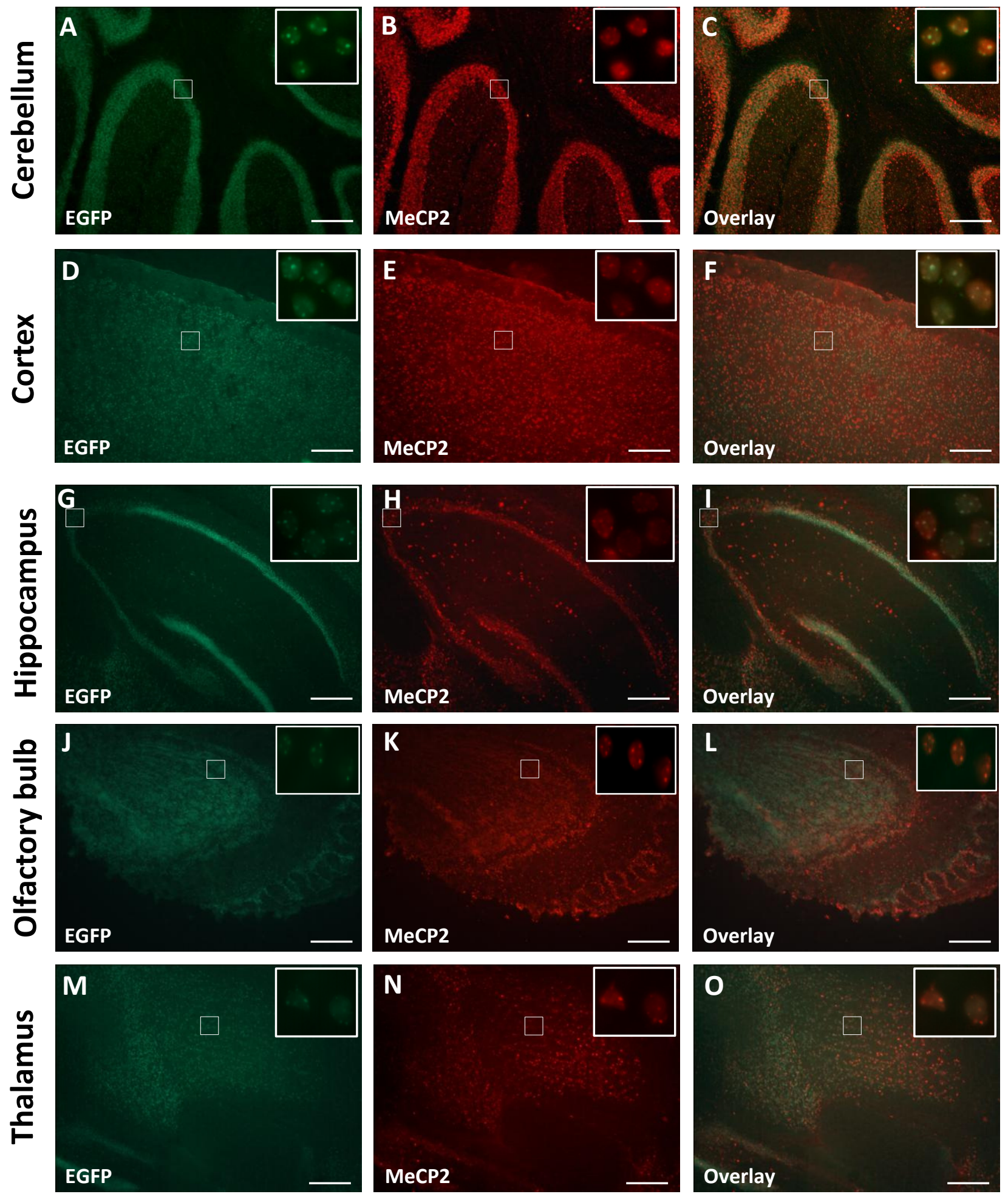

$\mathbf{N}$

MeCP2

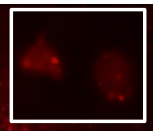

0

Overlay 
Figure: S3

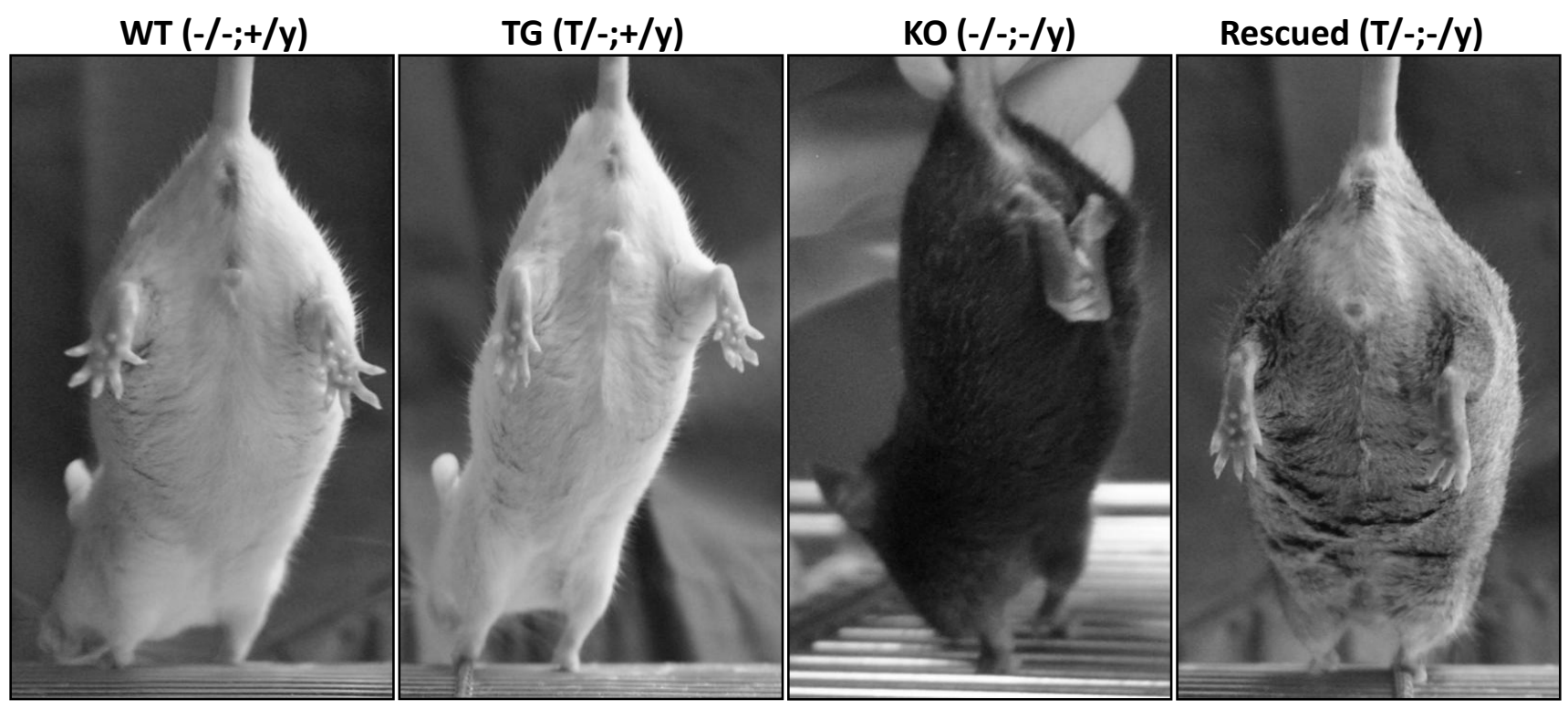


Figure: S4
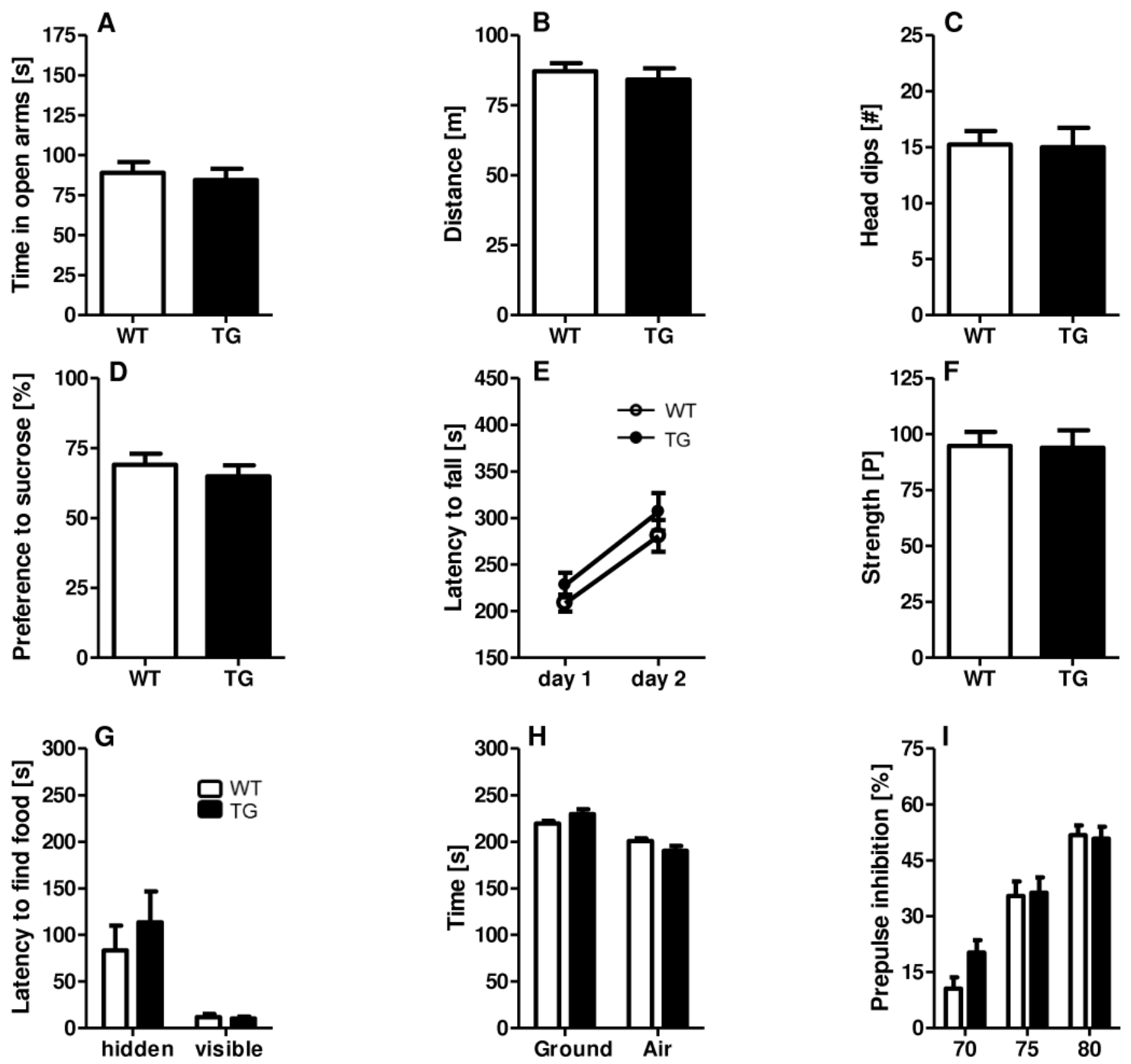

Prepulse intensity [db]

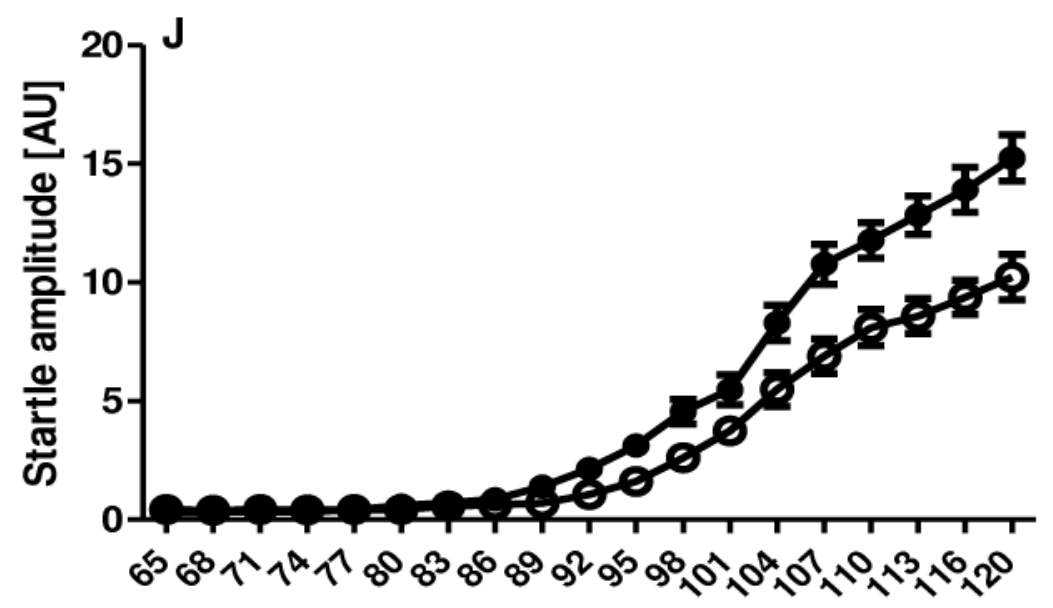


Figure: S5
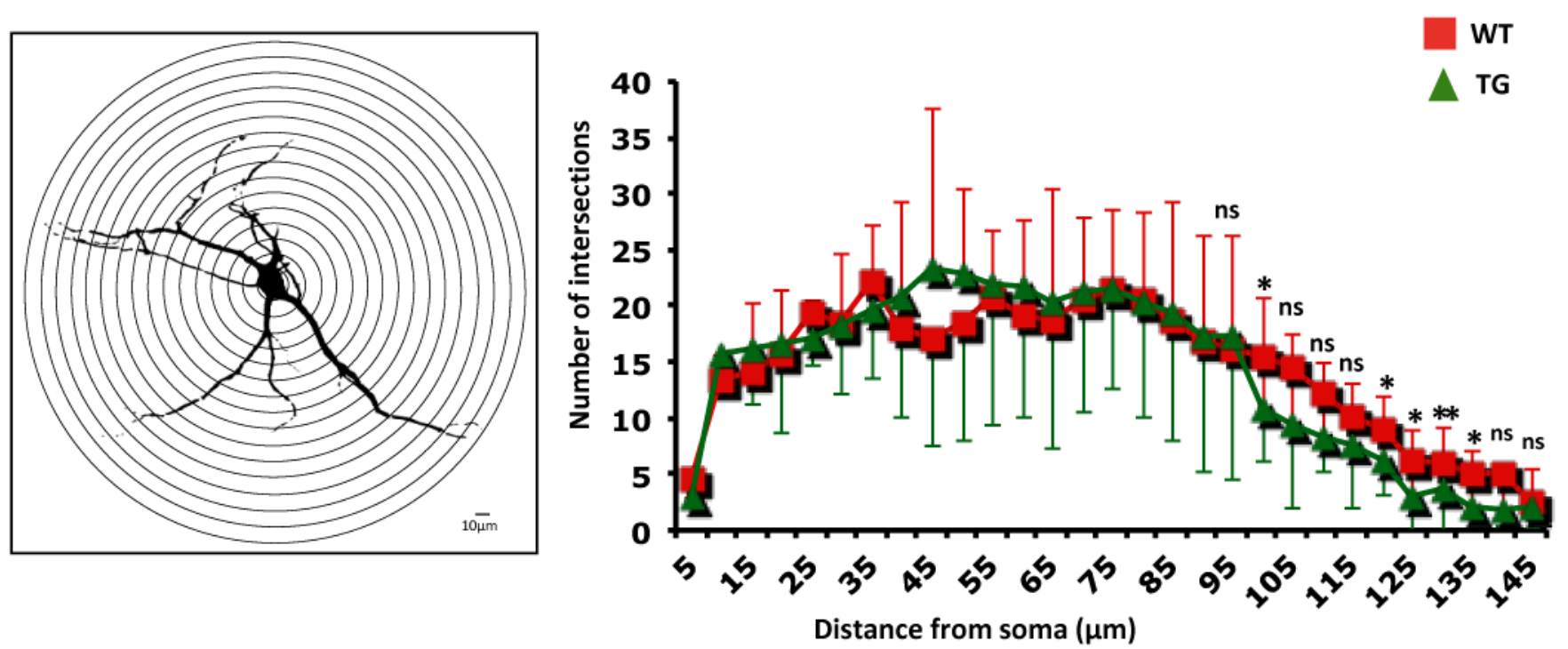
Supplemental Table S1: Neonatal assessment in transgenic mice.

\begin{tabular}{|l|l|l|}
\hline Domain & Behavorial Tests & WT vs TG \\
\hline \multirow{3}{*}{$\begin{array}{l}\text { Early postnatal physical } \\
\text { development }\end{array}$} & Body Weight & $\mathrm{p}>0.05$ \\
\cline { 2 - 3 } & Ear Opening & $\mathrm{p}>0.05$ \\
\cline { 2 - 3 } & Eye Opening & $\mathrm{p}>0.05$ \\
\hline $\begin{array}{l}\text { Early postnatal } \\
\text { neurodevelopment }\end{array}$ & Surface righting reflex & $\mathrm{p}>0.05$ \\
\cline { 2 - 3 } & Postural reflex & $\mathrm{p}>0.05$ \\
\cline { 2 - 3 } & Cliff avoidance & $\mathrm{p}>0.05$ \\
\cline { 2 - 3 } & Negative geotaxis reflex & $\mathrm{p}>0.05$ \\
\hline \multirow{2}{*}{$\begin{array}{l}\text { Early postnatal neuromotor } \\
\text { coordination }\end{array}$} & Wire suspension & $\mathrm{p}>0.05$ \\
\cline { 2 - 3 } & Swimming activity & $\mathrm{p}>0.05$ \\
\hline
\end{tabular}

Neonatal assessments were done covering the domains of physical development (body weight, the day of ear and eye opening), neurobehavioral development (surface righting, postural, cliff avoidance, and negative geotaxis reflex), and the development of neuromotor coordination (wire suspension and swimming activity).

Mice $(\mathrm{WT}=14, \mathrm{TG}=18)$. 


\section{MOUSE MODEL TARGETING A TRANSCRIPTIONAL REGULATOR, MECP2 (II):}

\section{Mild expression differences of MECP2 influencing aggressive social behavior}

\section{a. Overview of project III}

Results from mouse models carrying different Mecp2 mutations, ranging from loss-offunction to reduced or enhanced expression reveal a correlation between the dosage of Mecp2 and phenotype severity of the mutants, indicating a narrow threshold of the expression range, with deviance in both directions being disadvantageous (Chahrour and Zoghbi 2007; Chao and Zoghbi 2012). Previously shown in project I, mild overexpression of Mecp2 in the amount that exceeds wild-type levels by $\sim 1.5$ fold induced higher seizure propensity and aggression in male FVB/N mice, together with alterations in neuronal branching sites and augmented spine density (Bodda, Tantra et al. 2013). Also in vitro application of an epileptogenic compound, pentylenetetrazole to transgenic neurons led to a marked increase in amplitude and frequency of calcium spikes (Bodda, Tantra et al. 2013). Our findings are in line with the observation that the severity of the induced phenotypes increases not only as Mecp2 function decreases, but also as Mecp2 level increases beyond normal levels, delineating the necessity of a tight regulation of Mecp2 level to ensure proper functions (Chao and Zoghbi 2012).

While epileptic seizures are a well established pathological consequence of alterations in Mecp2 function/ expression (Collins, Levenson et al. 2004; Chahrour and Zoghbi 2007; Chao, Chen et al. 2010), the information on the functional consequences of mild overexpression on behavior is still sparse. Recent studies have shown that increasing the endogenous Mecp2 level up to two- and three-fold of physiological levels resulted in deficits in social interaction (Samaco, Mandel-Brehm et al. 2012). Along these lines, it has been shown that disturbed neuronal homeostasis across different Mecp2 mouse models has consistent social behavioral consequences (Pearson, Defensor et al. 2012). Here, the most common phenotypes observed are abnormal (enhanced/ impaired) social interaction, deficits in nest building and increased aggression (Shahbazian, Young et al. 2002; Moretti, Bouwknecht et al. 2005; Fyffe, Neul et al. 2008; Kerr, Alvarez-Saavedra et al. 2008; 
Samaco, Mandel-Brehm et al. 2009; Chao, Chen et al. 2010; Pearson, Defensor et al. 2012; Samaco, Mandel-Brehm et al. 2012). All together, there is growing evidence that Mecp2 regulates the expression/ function of genes involved in social behavior (Moretti, Bouwknecht et al. 2005).

It is worthwhile mentioning that the phenotypes observed across different mouse models arose from different genetic backgrounds of mutant mice carrying different type of Mecp2 mutations and therefore did not consider basic phenotypical differences among mouse strains. Behavioral studies on basic behavior, social and cognitive domains have revealed considerable phenotypical differences between different mouse strains (Wolfer and Lipp 2000; Moy, Nadler et al. 2009; Pietropaolo, Guilleminot et al. 2011; O'Leary, Gunn et al. 2013; Samaco, McGraw et al. 2013). For example, a strain-comparison study on $\mathrm{FVB} / \mathrm{N}$ and $\mathrm{C} 57 \mathrm{BL} / 6 \mathrm{~N}$ mice revealed that FVB/N mice showed higher frequency of bouts during the resident-intruder paradigm for aggression (Mineur and Crusio 2002; Pugh, Ahmed et al. 2004). It is therefore very likely that the genetic background could determine or modulate phenotypical changes induced by alterations in Mecp2 expression, and that particular phenotypes might be unique to a specific genetic background.

Being aware of the fact that genetic background might modulate the behavioral phenotype of mutant mice (Wolfer and Lipp 2000; Pietropaolo, Guilleminot et al. 2011; Samaco, McGraw et al. 2013), in the frame of the third project, transgenic mice with $\sim 1.5$ fold Mecp2 expression of the FVB/N were backcrossed for 10 generations to the C57BL/6N background to create a congenic strain. We then asked the question whether the symptoms that arose upon mild overexpression of Mecp2 are conserved across genetic backgrounds (FVB/N versus $\mathrm{C} 57 \mathrm{BL} / 6 \mathrm{~N}$ ) and genders. Including now female mice in behavioral analysis, we found that, independent of genetic backgrounds and genders, a marked increased in susceptibility to PTZ-induced was persistent in Mecp2 overexpressing mice. We also found that while mildly overexpressing Mecp2 at 1-5fold WT level in both FVB/N and C57BL/6N genetic backgrounds of both genders left most basic behavioral unaltered, it modulated spontaneous home cage activity.

Interestingly, we found that the territorial aggression of male mice with mild Mecp2 overexpression is genetic background-dependent. The marked reduction of the attack 
latency towards the intruder mice during the resident-intruder paradigm for territorial aggression was displayed only by transgenic mice of the $F V B / N$, but not the C57BL/6N background. We then investigated the pattern of territorial behavior of male transgenic mice of the C57BL/6N background and we found indicators of reduced territorial social interest in transgenic mice, compared to their WT littermates. However, observed in a neutral non-territorial arena (the 3-partite chamber), transgenic male mice of the C57BL/6N background showed normal pattern of social interaction. Furthermore, these transgenic male mice of the $\mathrm{C} 57 \mathrm{BL} / 6 \mathrm{~N}$ background displayed reduced nest building and lower ultrasound vocalization response to an anesthetized female intruder, and signs of social inferiority as assessed by social dominance test. Together, these data indicated that also in Mecp2 overexpressing mice of C57BL/6N background, territorial social behavior was a target phenotype. In contrast to TG mice of the FVB/N background, in these intrinsically less aggressive mice, however, aggression seemed to be dampened rather than intensified by Mecp2 overexpression. Thus, it appeared that while social behavior is susceptible to changes in Mecp2 level, the phenotypical alterations (i.e. more or less aggressive) depend on the behavioral traits of each genetic background.

Despite accumulating evidence on the importance of MECP2 for development and further maintenance of neuronal function (Guy, Cheval et al. 2011; McGraw, Samaco et al. 2011; Minh, Du et al. 2012), studies addressing the effect of common genetic variations of this gene on human behavior remain scarce. An association study on two collections of families showed a significant association between SNP single markers at the 3' end of MECP2 with autism/ autism spectrum disorder (Loat, Curran et al. 2008). Despite the necessity of further investigations, this study indicates that one/more functional variants of MECP2 existing at significant frequency in the population might influence the risk for autism/ autism spectrum disorders (Loat, Curran et al. 2008). In the acknowledgement of an overlap in behavioral phenotypes of RTT, MECP2 duplication syndrome and autistic individuals, there is the possibility that $M E C P 2$ variants might be involved in modulating behavioral domains affected across these disorders, such as social behavior. In the framework of the present project, we aimed to investigate whether slight MECP2 expression differences mediated by common genetic variations would be associated with behavioral phenotypes relevant for aggression, as observed in transgenic mice with mild overexpression of Mecp2. 
Our mouse studies revealed aggression as a central phenotype modulated by mild increased of Mecp2 expression. Accordingly, a phenotype-based genetic association study was conducted on two variants of MECP2: SNP rs2734647 (C/T) and rs2239464 (G/A). Phenotypical readouts such as poor impulse control, aggression, excitement and uncooperativeness were selected from the GRAS data collection (Ribbe, Friedrichs et al. 2010). We established that the selected readouts were substantially intercorrelated with each other, implying the likelihood of these items to represent behavioral expressions of overlapping neuronal circuitries. Significant associations between these readouts and both SNPs were found in male, but not female patients: male C-carriers of SNP rs2734647 and G-carriers of SNP rs2239464 were more aggressive.

To study the functional implications of the 3'UTR SNP rs2734647 (C/T) on microRNA (miR-) dependent posttranscriptional regulation of gene expression, in silico analyses were performed. Four miRs with seed binding sites including the 3'UTR SNP position were predicted. We found that in vitro luciferase assays using HEK293 cells demonstrated a significant reduction of luciferase activity upon co-transfection of the plasmid carrying the T-allele with miR-4711-3p and miR-511. When luciferase assays were performed using N2a cells, similar positive results were obtained for miR-511. Additionally, we further investigated whether allele-specific down-regulation of $M E C P 2$ expression would also be visible in peripheral blood mononuclear cells (PBMC) of male T-carriers of SNP rs2734647. Here we found a lower MECP2 expression (of about 50\%) in PBMC of male carrying T-allele, compared to C-carriers. All together, results from the third project suggest a role of Mecp2/MECP2 as a regulator of aggressive behavior in mouse and man, with genetic variations acting as a modifier.

\section{b. Submitted manuscript}

Tantra $\mathbf{M}^{*}$, Hammer $\mathrm{C}^{*}$, Kästner $\mathrm{A}^{*}$, Dahm $\mathrm{L}$, Begemann $\mathrm{M}$, Bodda $\mathrm{C}$, Hammerschmidt K, Giegling I, Stepniak B, Venzor AC, Konte B, Erbaba B, Hartmann A, Tarami A, Schulz-Schaeffer W, Rujescu D, Mannan AU, Ehrenreich H: Mild expression differences of MECP2 influencing aggressive social behavior. submitted.

${ }^{\star}$ Equal contribution 


\section{Own contribution:}

I was responsible for an extensive behavioral characterization of male and female mice with mild Mecp2 overexpression on both FVB/N and C57BL/6N genetic backgrounds. The behavioral study was conducted covering the domains of basic behavior, sensory, motor, cognitive functions and social behavior, partly including new test development and establishment. I further performed seizure induction experiments. I also did all the data analysis of the behavioral work and designed the figures relevant for the behavioral data (figure 1,2, 3 and 4 of the submitted manuscript). I wrote a part of the introduction of the manuscript, with the main focus on relevant animal studies on Mecp2. I also wrote the materials and methods and results sections of the manuscript. I interpreted the behavioral data and collected references and put them together in a context for the discussion part of the manuscript. 


\title{
Mild expression differences of MECP2 influencing aggressive social behavior
}

\author{
Martesa Tantra, M.Sc. ${ }^{1,2 *}$, Christian Hammer, Ph.D. ${ }^{1 *}$, Anne Kästner, Ph.D. ${ }^{1 *}$, \\ Liane Dahm, M.Sc. ${ }^{1}$, Martin Begemann, M.D. ${ }^{1}$, Chiranjeevi Bodda, M.Sc. ${ }^{2,3}$, \\ Kurt Hammerschmidt, Ph.D. ${ }^{4}$, Ina Giegling, Ph.D. ${ }^{5}$, Beata Stepniak, M.Sc. ${ }^{1}$, \\ Aracely Castillo Venzor, B.Sc. ${ }^{1}$, Bettina Konte, M.Sc. ${ }^{5}$, Begun Erbaba, B.Sc. ${ }^{1}$,
} Annette Hartmann, Ph.D. ${ }^{5}$, Asieh Tarami, M.Sc. ${ }^{1}$, Walter Schulz-Schaeffer, M.D. ${ }^{6}$, Dan Rujescu, M.D. ${ }^{5}$, Ashraf U. Mannan, Ph.D. ${ }^{2,3}$, and Hannelore Ehrenreich, M.D., D.V.M. ${ }^{1,2 \S}$

${ }^{*}$ Equal contribution

\footnotetext{
${ }^{1}$ Clinical Neuroscience, Max Planck Institute of Experimental Medicine, ${ }^{2}$ DFG Center for Nanoscale Microscopy \& Molecular Physiology of the Brain (CNMPB), ${ }^{3}$ Institute of Human Genetics, University Medical Center Göttingen, ${ }^{4}$ Cognitive Ethology Laboratory, German Primate Center, ${ }^{5}$ Department of Psychiatry, University of Halle, Halle, Germany ${ }^{6}$ Department of Neuropathology, University Medical Center Göttingen, Göttingen, Germany
}

Running title: $M E C P 2$ expression differences in aggression

Key words: Impulsivity, human, mouse, genetic background, transgenic, phenotypebased genetic association study, microRNA, SNP, PBMC, human autopsy brain tissue

\section{$\S^{\S}$ Correspondence:}

Professor Hannelore Ehrenreich, MD, DVM

Clinical Neuroscience

Max Planck Institute of Experimental Medicine

Göttingen, Germany

Phone +49-551-3899615

Fax +49-551-3899670

Email: ehrenreich@em.mpg.de 


\section{Tantra et al REVISED MANUSCRIPT}

\section{ABSTRACT}

The X-chromosomal MECP2/Mecp2 gene encodes methyl-CpG-binding protein 2, a transcriptional activator and repressor regulating many other genes. We discovered in male FVB/N mice that mild ( $50 \%)$ transgenic overexpression of Mecp2 enhances aggression. Surprisingly, when the same transgene was expressed in C57BL/6N mice, transgenics showed reduced aggression and social interaction. This suggests that Mecp2 modulates aggressive social behavior. To test this hypothesis in humans, we performed a phenotype-based genetic association study (PGAS) in >1000 schizophrenic individuals. We found MECP2 SNPs rs2239464 (G/A) and rs2734647 (C/T; 3'UTR) associated with aggression, with the G- and C-carriers, respectively, being more aggressive. This finding was replicated in an independent schizophrenia cohort. Allele-specific MECP2 mRNA expression differs in peripheral blood mononuclear cells by $\sim 50 \%$ (rs2734647: C>T). Notably, the brain-expressed, species-conserved miR-511 binds to MECP2 3'UTR only in T-carriers, thereby suppressing gene expression. To conclude, subtle MECP2/Mecp2 expression alterations impact aggression. While the mouse data provides evidence of an interaction between genetic background and mild Mecp2 overexpression, the human data convey means by which genetic variation affects MECP2 expression and behavior. 


\section{Tantra et al REVISED MANUSCRIPT}

\section{INTRODUCTION}

The X-chromosomal MECP2/Mecp2 gene encodes for methyl-CpG-binding protein 2, which can act both as a transcriptional activator and repressor (Chao \& Zoghbi, 2012). Indeed, hundreds of genes have been estimated to be regulated, directly or indirectly, by this protein (Chahrour et al, 2008; Cohen et al, 2008). Complete or partial loss-offunction mutations of MECP2 lead to Rett syndrome, characterized by a genderdependent array of symptoms, ranging from early loss of acquired speech and motor skills to severe mental retardation and neonatal encephalopathy, amongst many others (Amir et al, 1999; Bienvenu \& Chelly, 2006). Interestingly, gene duplication can cause very similar symptoms (Ramocki et al, 2010), and both down- and upregulation of MECP2 are associated with behavioral core features of autism spectrum disorders (ASD) (Peters et al, 2013).

Diverse genetic mouse models, ranging from complete loss-of-function to reduced or enhanced expression, have been generated to study consequences of Mecp2 mutations (Bodda et al, 2013; Chen et al, 2001; Collins et al, 2004; Guy et al, 2001; Moretti et al, 2005; Samaco et al, 2008; Shahbazian et al, 2002). In fact, there is a correlation between Mecp2 'dosage' and phenotype severity, indicating a narrow normal expression range, with deviance in both directions being disadvantageous (Chao \& Zoghbi, 2012). Along these lines, we have previously shown that mild overexpression of Mecp2 of 1.4-1.5 times the wildtype (WT) level induces increased seizure propensity and aggression in male FVB/N mice, together with alterations in neuronal branching sites and augmented spine density (Bodda et al, 2013). Application of an epileptogenic compound, pentylenetetrazole, to transgenic (TG) neurons in vitro leads to a marked increase in amplitude and frequency of calcium spikes (Bodda et al, 2013).

While epileptic seizure susceptibility is a well-established result of alterations in MECP2 expression (Bodda et al, 2013; Chahrour \& Zoghbi, 2007; Chao et al, 2010; Chao \& Zoghbi, 2012; Collins et al, 2004; Shahbazian et al, 2002), information on the behavioral consequences of mild overexpression is still rather limited. Doubling the Mecp2 expression level in mice resulted in impaired social interaction (Samaco et al, 2012). Mecp2 deficiency, however, also led to abnormal social behavior such as deficits in nest building, altered social interaction and enhanced aggression (Chao et al, 2010; Fyffe et al, 2008; Kerr et al, 2008; Moretti et al, 2005; Pearson et al, 2012; Samaco et al, 2008; Samaco et al, 2009; Shahbazian et al, 2002). Taken together, tightly regulated Mecp2 


\section{Tantra et al REVISED MANUSCRIPT}

expression is obviously critical for normal function of genes involved in social behavior (Moretti et al, 2005).

Importantly, the reported phenotypes arose from diverse genetic backgrounds of mice carrying Mecp2 mutations, not considering basic phenotypical differences among mouse strains (Moy et al, 2009; O'Leary et al, 2013; Pietropaolo et al, 2011; Samaco et al, 2013; Wolfer \& Lipp, 2000). For example, behavioral comparisons between FVB/N and $\mathrm{C} 57 \mathrm{BL} / 6 \mathrm{~N}$ strains revealed that FVB/N mice showed a higher frequency of bouts during behavioral paradigms of aggression (Mineur \& Crusio, 2002; Pugh et al, 2004). Thus, the genetic background might well mask or modulate phenotypical changes induced by alterations in Mecp2 expression.

In humans, data on aggression/impulsivity in Rett or MECP2 gene duplication syndrome are scarce. A family study characterizing neuropsychiatric phenotypes of 9 males and 9 females with MECP2 duplications revealed a high prevalence of hostility (63\%) in female carriers. Moreover, an asymptomatic Rett mutation carrier with the mutation located in the deletion hotspot of the MECP2 3' region has been reported to experience episodes of uncontrolled aggression (Huppke et al, 2006).

The present study has been designed (1) to explore the role of gender and genetic background (FVB/N versus C57BL/6N) for behavioral phenotypes associated with mild Mecp2 overexpression in mice, i.e. altered aggression, spontaneous home cage behavior and predisposition to epileptic seizures, and (2) to translate these findings to humans by searching for social behavioral consequences of common single nucleotide polymorphisms (SNPs) within MECP2 and (3) to strive for mechanistic insight into SNPrelated MECP2 expression differences in man.

\section{RESULTS}

Mild Mecp2 overexpression in FVB/N and C57BL/6N mice of both genders leaves most basic behaviors unaltered but modulates spontaneous home cage activity To estimate the expression of the TG protein Mecp2 ${ }^{\text {WT_EGFP }}(\sim 100 \mathrm{kDa})$ compared to endogenous Mecp2 ( 70kDa) in TG mice, we performed Western blot analysis with total 


\section{Tantra et al REVISED MANUSCRIPT}

protein extracted from hippocampus and cerebellum. As described previously for FVB/N mice (Bodda et al, 2013), the relative expression of Mecp2 ${ }^{\text {WT_EGFP }}$ in brain amounts to $40-50 \%$ of endogenous Mecp2 in both strains, leading to a subtle overexpression of 1.4-1.5-fold compared to WT. Mecp2 ${ }^{W T_{-} E G F P}$ TG mice of both genders and strains (FVB/N and C57BL/6N) develop and reproduce normally, and are devoid of any immediately obvious phenotype. Also, upon comprehensive testing of several independent cohorts of both genotypes and genders at different ages, all major domains of basic behavior, i.e. general activity, anxiety, motor functions, exploratory behavior, sensory and cognitive functions were unaltered when compared to the respective WT littermate controls (Table1a,b), thus confirming and extending our earlier report on male FVB/N TG mice (Bodda et al, 2013).

We wondered whether subtle behavioral differences upon mild overexpression of Mecp2 are perhaps not captured by the usual behavioral test battery. Therefore, spontaneous home cage behavior was monitored continuously overnight using LABORAS $^{\text {TM }}$. Indeed, we found significant differences between TG and WT mice. There was an overall tendency of reduced locomotion, including climbing, and increased immobility in TG as compared to WT, noticeable across strains and genders, except for male FVB/N mice that failed to show significant changes in locomotion (Figure1).

\section{Male Mecp2 transgenic mice of both genetic backgrounds exhibit altered territorial social behavior and aggression as compared to their WT littermates}

Based on the unexpected observation that mild overexpression of Mecp2 leads to increased territorial aggressive behavior of male TG versus WT FVB/N mice when exposed to FVB/N intruders (Bodda et al, 2013), we started here a series of new experiments on the resident-intruder paradigm: (1) We aimed at testing reproducibility of this phenomenon in $\mathrm{FVB} / \mathrm{N}$ mice exposed to younger intruder males of $\mathrm{C} 57 \mathrm{BL} / 6 \mathrm{~N}$ background, i.e. per se less aggressive intruders (Mineur \& Crusio, 2002; Pugh et al, 2004). This experiment yielded similar results (Figure2A), i.e. increased territorial aggression in TG FVB/N. (2) We were interested to see whether another genetic background, C57BL/6N, would modify the effect of Mecp2 overexpression. However, using the same resident-intruder protocol as in FVB/N males, no attack by C57BL/6N males was observed (Table1b). As male C57BL/6N mice are generally less aggressive 


\section{Tantra et al REVISED MANUSCRIPT}

compared to male FVB/N (Mineur \& Crusio, 2002; Pugh et al, 2004), the residentintruder protocol had to be slightly modified by extending the cut-off time to $10 \mathrm{~min}$ and increasing the basal level of aggression using warming (Gaskill et al, 2012; Greenberg, 1972). Even though the necessary increase in surrounding temperature $\left(<38^{\circ} \mathrm{C}\right)$ is far below the temperature used to test pain sensitivity (hot plate test; temperature set to $55^{\circ} \mathrm{C}$ ), we note here that the pain threshold in TG and WT mice was identical (Table $1 \mathrm{~b}$ ). Prior to testing, the home cage of the resident mouse was placed under a heat-emitting red lamp for $20 \mathrm{~min}$ to obtain mild prewarming. Further, during testing, the home cage of the resident was positioned on a warming pad (set to $38^{\circ} \mathrm{C}$ ). Surprisingly and opposite to $\mathrm{FVB} / \mathrm{N}$ mice, the attack latency of $\mathrm{TG} \mathrm{C} 57 \mathrm{BL} / 6 \mathrm{~N}$ was longer, pointing to reduced territorial aggression as compared to WT littermates. In fact, within the $10 \mathrm{~min}$ observation, $30 \%$ of WT and $55 \%$ of TG residents of the C57BL/6N background did not attack the intruder at all (Figure2A). In contrast to the clear alteration of territorial aggression in TG versus WT male mice of both strains, the sociability test (mouse preferred over empty cage) revealed entirely normal behavior, indistinguishable between TG and WT (Figure2B).

The unforeseen result of reduced aggression in male TG C57BL/6N prompted us to investigate the pattern of their territorial behavior during the resident-intruder test. We measured frequency and duration of agonistic encounters of the resident mouse for the first $3 \mathrm{~min}$ upon introducing the intruder. Whereas the latency to initiate the first contact was comparable between male WT and TG C57BL/6N mice $(8.5 \pm 1.3 \mathrm{~s}$ versus $10.7 \pm 1.9 \mathrm{~s} ; \mathrm{p}=0.35$ ), TG mice exhibited lower frequency and duration of follow/chase behavior, as well as sniffing of facial and anogenital areas of the intruder mice than their WT littermates (Figure3).

Since this territorial behavioral pattern of male C57BL/6N TG mice indicated somewhat reduced social interest, we hypothesized that these mice might also display other signs of changed social interest/competence. Indeed, nest building capacity/quality, social dominance measured by the tube test, and ultrasound vocalization in response to an anesthetized female intruder, revealed inferiority or at least a strong tendency thereof in TG animals (Figure3). Together, these data indicate that also in Mecp2 overexpressing mice of $\mathrm{C} 57 \mathrm{BL} / 6 \mathrm{~N}$ background, territorial aggressive behavior is a central target phenotype. In these intrinsically non-aggressive mice, however, the direction of change 


\section{Tantra et al REVISED MANUSCRIPT}

is exactly opposite to the FVB/N strain and accompanied by reduced social interest and competence.

Seizure propensity is increased upon mild Mecp2 overexpression, independent of genetic background and gender

A common characteristic neurological phenotype found in different mouse models of mutant Mecp2, ranging from complete loss-of-function to overexpression, are epileptic seizures (Chahrour \& Zoghbi, 2007; Collins et al, 2004; Guy et al, 2001; Shahbazian et al, 2002). We previously reported in male FVB/N TG mice a higher susceptibility to seizure induction by the $\mathrm{GABA}_{A}$ receptor antagonist, pentylenetetrazole (PTZ) (Bodda et al, 2013). This work could now be expanded to female FVB/N TG as well as to male and female C57BL/6N TG mice. Indeed, PTZ-induced seizure propensity is increased across genetic backgrounds and genders (Figure4), pointing to a strong overall phenotypical consequence of mild Mecp2 overexpression.

The startle response to acoustic stimulation is augmented upon mild Mecp2 overexpression in female mice of both genetic backgrounds

Deletion of Mecp2 in GABAergic neurons resulted in reduction of the startle response to acoustic stimuli of $120 \mathrm{db}$ (Chao et al, 2010), but nothing has been known regarding a potential influence of mild Mecp2 overexpression on startling. Since Mecp2 TG mice of both genetic backgrounds exerted an exaggerated seizure reaction to a $\mathrm{GABA}_{\mathrm{A}}$ receptor antagonist, PTZ, we wondered whether mild overexpression of Mecp2 might also alter the startle reflex and/or the prepulse inhibition of the startle response (PPI). Indeed, we found enhanced startling in female but not male Mecp2 TG mice of both FVB/N and C57BL/6N background (Figure4). No changes in the percentage of PPI were observed in any strain or gender (Table1a,b). Similar findings that the startle response at $120 \mathrm{db}$ does not necessarily affect overall PPI were reported in the context of a study on mouse inbred strain differences (Paylor \& Crawley, 1997). 


\section{Tantra et al REVISED MANUSCRIPT}

\section{MECP2 SNP distribution is comparable in healthy and schizophrenic individuals}

Since our mouse studies revealed aggressive social behavior as a central phenotype modulated by subtle Mecp2 overexpression, we started a hypothesis-driven analysis on subjects of the GRAS (Göttingen Research Association for Schizophrenia) data collection (Ribbe et al, 2010). Genotyping for association analyses was performed for 4 SNPs located in the X-chromosomal MECP2 gene with reasonably high minor allele frequency (Figure5A). At first, a potential genetic risk condition of the selected SNPs for schizophrenia was assessed: A case-control study comparing allele frequencies of the 4 genotyped SNPs rs2239464, rs3027933, rs2075596, and rs2734647 in 1052 GRAS patients versus 1248 healthy controls was performed separately for men and women. All markers fulfilled Hardy-Weinberg criteria, and no significant associations with diagnosis were detected (Figure5B,C). Due to strong linkage disequilibrium (LD) between markers, only rs2239464 and rs2734647 were considered for the phenotypebased genetic association study (PGAS, inclusion criterion $\left.r^{2}<0.8\right)$ (Figure5D).

\section{Normal genetic variation of MECP2 influences aggression and impulsivity in man}

Translating our findings in mild Mecp2 overexpressing mouse models to humans, we selected aggression-related variables from the GRAS phenotypical data collection and tested their association with the 2 selected MECP2 SNPs separately in men and women. Poor impulse control and a further trait reflecting behavioral expression of impulsive aggression (excitement) were chosen. For both, SNP rs2734647 in the 3'UTR (Table2), as well as for SNP rs2239464 (Table3), significant associations with these traits were found in men, with C-carriers and G-carriers (the major alleles), respectively, being more aggressive. Sociodemographic and clinical measures potentially confounding these results did not differ between genotype groups. The nominally significant result for years of education in males (SNP rs2734647) was accounted for by including it as a covariate in all models with target measures as dependent variables. In contrast, for women, no statistically significant results were obtained. The phenotypegenotype relationships could be reproduced in a small independent sample of schizophrenic men (Table4). Taken together, the association of MECP2 genotypes with readouts of aggression, as detected in mice, was confirmed in two independent cohorts of men. 


\section{Tantra et al REVISED MANUSCRIPT}

\section{SNP rs2734647 in the 3'UTR affects miR-511 binding and gene expression}

To examine possible functional implications of SNP rs2734647 in the 3'UTR on microRNA (miR)-dependent regulation of gene expression, in silico analyses were performed using TargetScan (Release 6.2) (Grimson et al, 2007; Lewis et al, 2005) and PITA (Kertesz et al, 2007), resulting in the prediction of 4 miRs with seed binding sites

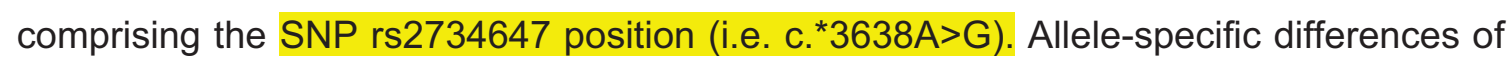
the predicted $\Delta \Delta G$ values, indicating strength of $\mathrm{miR}$ binding, are summarized in Figure6A. While miR-4711-3p and miR-511 are predicted to show preferential binding in case of the presence of the T-allele, miR-515-3p has a strong negative $\Delta \Delta G$ only in case of the C-allele, and miR-519e lacks a strong allele preference. In vitro luciferase assays using HEK293 cells revealed significantly reduced luciferase activity in case of co-transfection of the plasmid carrying the T-allele with both miR-4711-3p and miR-511 (Figure6B). Co-transfection with miR-515-3p or miR-519e did not lead to luciferase activity reduction for either rs2734647 T or C (Figure6B). In luciferase assays using N2a cells, the positive result was only replicated for miR-511 (Figure6C). In summary, these data strongly suggest miR-511 as rs2734647 genotype-dependent candidate for MECP2 regulation in humans. For the sake of completeness, we determined endogenous expression of miR-511 in the cell lines used for transfection. Whereas mmu-miR-511 levels were under the detection limit in N2a cells, hsa-miR-511 was clearly expressed in HEK293 cells $\left(3.59^{*} 10^{-3}\right)$. However, since we always used negative, i.e. non-miR-transfected controls, Luciferase assay results are unlikely to have been affected.

\section{miR-511 is expressed in aggression-relevant human brain regions}

Since miR-511 seems to be an important modulator of rs2734647 genotype-dependent MECP2 expression, we asked whether this miR would be detectable in brain regions relevant for impulsivity and aggression (frontal and pre-frontal cortex, temporal cortex, occipital cortex, hippocampus, and amygdala). Placenta was used as developmental control tissue. In all regions examined, miR-511 expression was found (Figure6D). Importantly, the same holds true for MECP2 using primers amplifying either MECP2_e2 only or both isoforms (Figure6E). In contrast, miR-4711-3p expression was not detected 


\section{Tantra et al REVISED MANUSCRIPT}

(data not shown), at least questioning a role of this miR for MECP2 regulation in the adult human brain. In the adult C57BL/6N versus FVB/N mouse brain, the expression of miR-511 was low and comparable between strains in cortex $\left(2.39 * 10^{-5}\right.$ versus $3.71^{*} 10^{-}$ $\left.{ }^{5}\right)$, hippocampus $\left(6.45^{*} 10^{-5}\right.$ versus $\left.3.78^{*} 10^{-5}\right)$, and cerebellum $\left(2.22^{*} 10^{-5}\right.$ versus $2.36^{*} 10^{-}$ $\left.{ }^{5}\right)$. Strong expression was found in embryonic tissue (C57BL/6N embryo head E17: $1.83^{*} 10^{-3}$; embryo body: $\left.2.26^{*} 10^{-3}\right)$, and highest in cultured microglia $\left(13.02^{*} 10^{-3}\right)$.

\section{Peripheral blood mononuclear cells (PBMC) of male rs2734647 T-carriers show lower MECP2 expression}

Since miR-511 was shown to be expressed in dendritic cells and macrophages (Tserel et al, 2011), we investigated the hypothesized allele-specific down-regulation of MECP2 expression in PBMC of male subjects. Indeed, a significantly lower expression in Tcarriers versus C-carriers (amounting to around 50\%) was detected with primers amplifying both MECP2 isoforms, whereas MECP2_e2 alone showed no expression difference dependent on the rs2734647 genotype (Figure6F). This result may indicate that in T-carriers mainly the MECP2_e1 isoform is affected, at least in PBMC, potentially related to isoform-specific different lengths of the MECP2 3'UTR (Coy et al, 1999).

\section{Sequence variation of miR-511 in mouse and man underlines the importance of an interaction with the MECP2 3'UTR}

A perfect seed match of human hsa-miR-511 with the MECP2 3'UTR in carriers of rs2734647-T is the most likely reason for the difference in luciferase activity in contrast to the $\mathrm{C}$-allele, which results in a mismatch in the seed binding region. $\mathrm{C}$ is the conserved ancestral allele, found in multiple other mammalian species, including mouse. Strikingly, the seed sequence of mouse mmu-miR-511 differs from hsa-miR-511 exactly regarding the nucleotide complementary to the SNP position (Figure6G). Thus, mmu-miR-511 shows an ideal seed match to the mouse 3'UTR, whereas hsa-miR-511 perfectly fits to the human 3'UTR carrying the T-allele. This observation may emphasize the importance of the miR-511 interaction with MECP2.

Notably, screening of available data from Jackson Laboratories (http://www.jaxlab.org) revealed that the two mouse lines employed here are not polymorphic for the Mecp2 


\section{Tantra et al REVISED MANUSCRIPT}

3'UTR allele or the miR-511 sequence. It would, however, be interesting to investigate whether non-inbred mice are polymorphic for the respective alleles.

\section{DISCUSSION}

The present study, extending from mouse to man, shows that mildly increased MECP2 expression leads to alterations in male social aggression. Using C57BL/6N versus FVB/N mice (with their known inherent strain differences in aggressive behavior) (Mineur \& Crusio, 2002; Pugh et al, 2004) as models, we demonstrate that the direction of change upon mild Mecp2 overexpression in this behavioral target domain is subject to modification by the genetic background. In other words, the resulting lower or higher social aggression depends on the basic genetic make-up of a particular subject. The discovery of MECP2 influencing aggressive behavior could also be reproduced in two independent cohorts of schizophrenic men, with a polymorphism in the 3'UTR of the gene co-determining both the level of MECP2 expression as well as of aggression. The genotype-dependent expression difference in men found in PBMC (around 50\%) is in the range of the transgenic overexpression in both mouse strains, emphasizing the physiological significance of these findings as well as of our mouse models for studying behavioral consequences of the normal 'Mecp2 dose range'.

Even though in both schizophrenia samples, higher expression of MECP2 (3'UTR SNP rs2734647 C carriers lack suppressability by miR-511) was associated with higher aggression, it has to be considered that also humans are not an isogenic population. One might thus, in analogy to our observation in different mouse strains, predict a human population with reduced aggression as suggested by the $\mathrm{C} 57 \mathrm{BL} / 6 \mathrm{~N}$ data (i.e. a bimodal aggression distribution when combining different genetic backgrounds).

Although the results for women show a similar tendency for poor impulse control and excitement as aggression readouts, they are far from reaching significance, likely due to the smaller number of individuals, but certainly also to the fact that MECP2 is $\mathrm{X}$ chromosomal. Homozygous $T$ carriers in the female sample are therefore expectedly very rare. 


\section{Tantra et al REVISED MANUSCRIPT}

In our translational PGAS approach, we had the chance to explore the aggression association of MECP2 genotypes in a phenotypically very well characterized large sample of schizophrenic individuals (Ribbe et al, 2010), and we replicated the association findings in a second, independent cohort of schizophrenic men. Therefore, we cannot state with certainty at this point, that the phenotype association holds true in the same way for healthy individuals. Nevertheless, apart from the supporting data obtained for healthy mice, it has to be emphasized that aggression is not a specific or unique symptom in schizophrenia. Also, the range of aggressive features in the whole GRAS patient sample follows a normal distribution, extending from very low to very high aggression scores.

Along the same lines, the case-control study presented here, including 1052 cases and 1248 controls, fails to attribute to MECP2 any schizophrenia risk gene role. In some contrast, a recent study reported in a Han Chinese population an association of rs2734647 C with the disease (498 cases versus 2025 controls, replicated in 1027 cases versus 1005 controls) (Wong et al, 2013). Although we did not even find a respective trend (Figure5C), we cannot entirely exclude limited power of our casecontrol approach. On the other hand, the association might well be population-specific. In any case, a potential risk gene status, even if confirmed by future GWAS including Xchromosomal genotypes, will not be dramatic considering the large number of individuals needed for its demonstration. Instead, MECP2 is most likely diseaseindependently involved in the regulation of a basic mammalian behavioral phenotype, i.e. aggression. Interestingly, carriers of the minor allele of MECP2 SNP rs2239464 were previously shown to have decreased cortical surface area in brain regions such as the cuneus (Joyner et al, 2009), which is associated with inhibitory control in patients with bipolar disorder (Haldane et al, 2008).

In further support of an association between MECP2 and aggression, impulse control alterations in individuals with MECP2/Mecp2 gene duplication syndrome have been reported (Ramocki et al, 2009), even though the findings of the present study are more relevant for the understanding of physiological gene-dose effects on social behavior. Importantly, Mecp2/MECP2 functions as transcriptional regulator targeting hundreds of other genes (Ben-Shachar et al, 2009; Bird, 2008; Chahrour et al, 2008; Chen et al, 


\section{Tantra et al REVISED MANUSCRIPT}

2003; Sun \& Wu, 2006; Wu et al, 2010). Thus, it is most likely a whole pattern of genes directly or indirectly influenced by this regulator - that primes nuances of aggressive social behavior. As an example, changes in the expression of a Mecp2 regulated gene, Prom1, are associated with domestication and aggressive behavior in animals (Albert et al, 2012; Gopisetty et al, 2012). Furthermore, Mecp2 is known to control expression of brain-derived neurotrophic factor (Bdnf) (Martinowich et al, 2003), which in turn is involved in the regulation of aggression (Ito et al, 2011).

Aggression seems to be a strong target phenotype of mild Mecp2/MECP2 overexpression independent of the genetic background, since these expression changes did not lead to alterations in basic behavior, including motor, sensory and cognitive functions. Male and female TG mice of both the FVB/N and C57BL/6N genetic backgrounds displayed basic behavior comparable to their WT littermates. Apart from aggression, only home cage activity, seizure propensity and startle response were influenced by mild Mecp2 overexpression in a fashion widely independent of the genetic background.

There have been reports on sexual dimorphism with respect to Mecp2 expression and function in the brain. For instance, in amygdalae and ventromedial hypothalamus, male rats express less Mecp2 as compared to females ((Kurian et al, 2008; Kurian et al, 2007). Furthermore, conditional knockout of Mecp2 during amygdala development caused subtle modifications of juvenile play behavior in male but not female rats (Kurian et al, 2008). These findings may indicate a role of Mecp2 in gender-specific modulation of behavior. In the present work, however, sexual dimorphism was consistently observed only with the startle response in a genotype and genetic background independent manner.

Even though the explicit situations are still unknown in which miR-511 regulated MECP2/Mecp2 expression might be of particular physiological relevance, any kind of inflammation in the brain for instance could play a pivotal role, considering the relatively high expression found here in mouse microglia. The distinct suppression of MECP2 expression by miR-511 in SNP rs2734647-T carriers reported here may even be considered as a future treatment target in MECP2 gene duplication syndrome. In any case, the high conservation of the interaction between miR-511 and MECP2 in both 


\section{Tantra et al REVISED MANUSCRIPT}

mouse and man makes a specific significance of their interplay very likely. This significance is further supported by the here demonstrated co-expression of MECP2 and miR-511 in human brain areas pivotal for aggression and impulsivity regulation (Bauman et al, 2006; Berlin et al, 2004; Brower \& Price, 2001; Horn et al, 2003; Siever, 2008; Whelan et al, 2012; Zetzsche et al, 2007). Interestingly, miR-511 expression was found here also in different mouse brain areas, with levels comparable across both genetic backgrounds.

To conclude, MECP2/Mecp2 has been shown here to be a regulator of social aggressive behavior in mouse and man, with the genetic background playing an important modifier role.

\section{MATERIALS \& METHODS}

\section{Mice}

All mouse experiments have been approved by the Animal Care and Use Committee of Lower Saxony, Oldenburg, Germany. The generation of Mecp2 $2^{W T_{-} E G F P}$ TG mice with a 1.4-1.5-fold Mecp2 overexpression on FVB/N background has been described in detail previously (Bodda et al, 2013). Briefly, a bacterial artificial chromosome (BAC) clone, pBAC_B22804, containing $120 \mathrm{~Kb}$ of murine genomic fragment with the intact Mecp2 gene and the flanking Opsin1 and Irak1 genes was used for generating the transgenic construct (Kifayathullah et al, 2010). To generate the pBAC_Mecp2WT_EGFP construct, the enhanced green fluorescent protein/kanamycin-resistant gene (EGFP/kan) cassette was PCR amplified using pEGFP1 vector as template with primers containing $50 \mathrm{bp}$ flanking sequence from either side of the Mecp2 stop codon. The endogenous stop codon was replaced by two glycine residues inframe between the MeCP2 protein and the EGFP protein to facilitate the two proteins to fold and function independently. The amplified EGFP/Kan cassette was electroporated into E.coli harboring the BAC clone and PGET recombination system, to facilitate the homologous recombination of EGFP/Kan cassette at the site of stop codon of Mecp2. The correct insertion of EGFP/Kan cassette after the recombination event into the BAC clone was confirmed by sequencing. The Mecp2 flanking genes, Opsin1 and Irak1 were deleted 


\section{Tantra et al REVISED MANUSCRIPT}

from the modified BAC clone using additional BAC recombineering with zeocin selection cassette (containing the BAC homology arms and zeocin antibiotic marker gene driven by EM7 promoter frompSELECT vector) (InvivoGen, Toulouse, France), to avoid any additional phenotype arising from the overexpression of these genes. During the process of Opsin1 deletion, a Mlul restriction site was introduced into BAC clone. The final BAC construct pBAC_Mecp2WT_EGFP was linearized with Mlul restriction enzyme and micro-injected into the male pronuclei of the fertilized mouse oocytes derived from the FVB/N strain. Next, the injected oocytes were transplanted into the uteri of the foster mothers. Genomic DNA isolated from tail biopsies was analyzed for the presence of the transgene by PCR. Because the transgene was not confirmed to be localized to the $X$ chromosome, translational relevance with respect to modelling mosaicism resulting from $\mathrm{X}$ chromosome loss is limited.

To create a comparator congenic strain for behavioral analyses, Mecp2 TG FVB/N mice were backcrossed for 10 generations to the $\mathrm{C} 57 \mathrm{BL} / 6 \mathrm{~N}$ background. For experiments reported here, male and female Mecp2 $2^{W T_{-} E G F P}$ TG (hemizygous) and their WT littermates on either FVB/N or C57BL/6N backgrounds were used. Tail biopsies were taken before weaning to obtain genomic DNA for genotyping (Bodda et al, 2013; Kifayathullah et al, 2010). Western blot and qPCR analyses for TG expression estimation was performed as described previously (Bodda et al, 2013).

\section{Behavioral analyses}

After weaning and during the whole period of behavioral testing, mice were housed individually in standard plastic cages $(26.5 \times 20 \times 14 \mathrm{~cm})$ and kept under temperaturecontrolled environment $\left(21 \pm 2^{\circ} \mathrm{C}\right)$ on $12 \mathrm{~h}$ light/dark cycle with food and water ad libitum, unless stated otherwise. Single housing was necessary since male FVB/N mice exhibited extremely aggressive behavior in a group-housed setting. In order to avoid housing differences as confounding variables, we decided to single-house all mice, independent of gender and strain. All experiments were conducted by investigators unaware of the genotype ('blinded'), during the light phase of the day (between 8:00am and $6 \mathrm{pm}$, except for automated home cage behavioral assessment. Several independent cohorts of mice (genders and strains tested separately, starting at 5 weeks of age) were run through a battery of tests covering altogether basic behavioral, sensory, motor, cognitive and social functions (for overview see Table1a,b). The order 


\section{Tantra et al REVISED MANUSCRIPT}

of tests was always oriented toward increasing invasiveness and performed as published in detail earlier (Bodda et al, 2013; El-Kordi et al, 2012; Jamain et al, 2008): Elevated plus maze (anxiety), open field (spontaneous activity), hole board (exploratory behavior), grip strength and rotarod (motor force, balance and coordination), marble burying (stereotypies and obsessive-compulsive behaviors), prepulse inhibition of the startle response (sensorimotor gating), hearing test (startle curve upon random presentation of stimulus intensities from $65 \mathrm{~dB}$ to $120 \mathrm{~dB}$ ), Y-maze (working memory), novel object recognition, visual cliff (vision), sociability (social preference, i.e. other mouse over object), buried food finding (olfaction), sucrose preference (anhedonia), hot plate test (pain sensation), and hole board (working and reference memory). Moreover, automated home cage behavior analysis (LABORAS ${ }^{\mathrm{TM}}$ ), ultrasound vocalization recording, nest building (social competence), social tube (dominance), resident-intruder paradigm (aggression) and seizure propensity (seizure induction by pentylenetetrazole) were performed as indicated (Table1a,b). In the following, the here relevant tests (with significant results) will be described in detail, while for the other tests the reader is politely referred to previous publications of ourselves and others (Bodda et al, 2013; ElKordi et al, 2012; Jamain et al, 2008; Kuc et al, 2006; Mandillo et al, 2008).

Automated home cage behavior analysis was performed using LABORAS ${ }^{\mathrm{TM}}$ system (Metris b.v., Hoofddorp, The Netherlands) which consists of a triangular shaped sensor platform (Carbon Fiber Plate $1000 \mathrm{~mm} \times 700 \mathrm{~mm} \times 30 \mathrm{~mm}$ ), positioned on 2 orthogonally placed force transducers (Single Point Load Cells) and a third fixed point attached to a heavy bottom plate (Corian Plate $980 \mathrm{~mm} \times 695 \mathrm{~mm} \times 48 \mathrm{~mm}$ ). The whole structure stands on 3 spikes, which are adjustable in height and absorb external vibrations. Mice are housed in clear polycarbonate cages (Makrolon type II cage, $22 \mathrm{~cm} \times 16 \mathrm{~cm} \times 14 \mathrm{~cm}$ ) with wood-chip bedding covered floors. The cage is placed directly onto the sensing platform, with the upper part of the cage (including top, food hopper and drinking bottle) suspended in a height-adjustable frame separate from the sensing platform. Resultant electrical signals caused by mechanical vibrations as induced by movement of the mouse are transformed by each force transducer, amplified to a fixed signal range, filtered to eliminate noise, digitized and stored on a computer. Stored signals are classified into separate behavioral categories like locomotor activity, immobility and climbing, and quantified by the LABORAS ${ }^{\mathrm{TM}}$ software. Prior to each session, LABORAS $^{\text {TM }}$ was calibrated. Spontaneous mouse behavior was assessed from 6:00pm 


\section{Tantra et al REVISED MANUSCRIPT}

until 9:00am, with $1 \mathrm{~h}$ cage habituation prior to initiation of recording. Male (FVB/N: 10TG, 10WT; C57BL/6N: 12TG, 24WT) and female mice (FVB/N: 10TG, 10WT; C57BL/6N: 13TG, 28WT) were tested.

Ultrasound Vocalizations (USVs) were recorded using a microphone (UltraSoundGateCM16) connected to a preamplifier (UltraSoundGate116), which was linked to a computer. At the day of recording, mice in their home cage (single housed) were placed in the recording room for 60s. Subsequently, the intruder mouse was put into the resident's cage, and vocalization behavior recorded for $3 \mathrm{~min}$. The intruder mouse was an anaesthetized unfamiliar female (anesthetic: intraperitoneal injection of $0.25 \%$ tribromoethanol, $0.1 \mathrm{ml} / 10 \mathrm{~g}$ body weight). Number of calls per recording session was counted and USVs were separated from other sounds using the whistles detection algorithm of Avisoft-SASLab 5.2 with following selection criteria: Possible changes per step $=4(4687 \mathrm{~Hz})$, minimal continuity $=8 \mathrm{~ms}$, possible frequency range $=35-150 \mathrm{kHz}$. These criteria had been tested in former studies of mouse USVs (El-Kordi et al, 2012; Hammerschmidt et al, 2012). Avisoft Bioacoustics, Berlin, Germany, delivered all sound recording hardware and software. Male C57BL/6N mice (12TG and 23WT) were tested.

The resident-intruder test was used to study inter-male aggression in various independent cohorts of TG and WT mice of both genetic backgrounds (FVB/N and C57BL/6N) and different age groups, ranging from 8-32 weeks (Bodda et al, 2013; Jamain et al, 2008). As standard opponents (intruders), group-housed males (4 weeks younger than resident test males) of C57BL/6N background were employed (Charles River, Sulzfeld, Germany). An intruder was introduced into the home cage of the test resident. Observation started when the resident first sniffed the opponent and stopped (stop watch) at first attack (defined as bite) to prevent wounding, but lasted for $300 \mathrm{~s}$ (FVB/N) or 600s (C57BL/6N) if no attack occurred (cut-off) (Mineur \& Crusio, 2002; Pugh et al, 2004). Male mice (FVB/N: 12TG, 22WT; C57BL/6N: 10TG, 13WT) were tested. Over the first 180s (unless mice attacked before), frequency and duration of following behaviors was additionally quantified in C57BL/6N (10TG, 11WT): nose/snout and anogenital sniff, following/chasing.

The social tube test measures social dominance (Garfield et al, 2011; Messeri et al, 1975; Moretti et al, 2005). The test apparatus comprises a $30 \mathrm{~cm}$ long transparent acrylic 


\section{Tantra et al REVISED MANUSCRIPT}

tube with an internal diameter of $3 \mathrm{~cm}$. Two mice are placed from opposite ends in the tube and gently pushed to the middle, where they face each other closely. Singlehoused TG and WT C57BL/6N mice were challenged with unrelated group-housed mice of C57BL/6N background. A subject was declared 'winner' when its opponent completely retreated from the tube ('out') within 300s (cut-off). To account for both, winning/losing and time to win/lose, dominance values are calculated using the following formulas: winner dominance value=100/time to out and loser dominance value = 1/(300-time to out). Male C57BL/6N mice (10TG, 16WT) were tested in this paradigm.

Nest building is an important behavior in rodents, reflecting social competence in reproduction (Deacon, 2006; El-Kordi et al, 2012; Satoh et al, 2011). Two hours before dark phase, the nesting towel was removed from home cages of single-housed mice and replaced by a nestlet (pressed $2.7 \mathrm{~g}$ cotton square). Nest building quality was scored in the morning (Deacon, 2006; El-Kordi et al, 2012). Male C57BL/6N mice (10TG, 16WT) were tested.

The pentylenetetrazole-induced seizure protocol has been described in detail previously (Bodda et al, 2013; Ferraro et al, 1999; Wojcik et al, 2013). Seizure activity was induced in wakeful mice by a single intraperitoneal (i.p.) injection of pentylenetetrazole (PTZ) $(50 \mathrm{mg} / \mathrm{kg}$ body weight) followed by close observation for $30 \mathrm{~min}$ in a small, clear homecage. Latencies to focal (partial clonic), generalized clonic and maximal tonic-clonic behavioral seizures were recorded. Furthermore, 4 phases in the continuum of behavioral response to PTZ injection were defined as follows: (1) Hypoactivity (progressive decrease in motor activity until resting in a crouched or prone position with abdomen in full contact with cage bottom); (2) Partial clonus (clonus seizure activity affecting face, head, and/or forelimb or forelimbs); (3) Generalized clonus (sudden loss of upright posture, whole body clonus involving all 4 limbs and tail, rearing and autonomic signs); (4) Tonic-clonic (maximal) seizure (generalized seizure characterized by tonic hindlimb extension - also associated with death). Finally, latencies to partial clonus (PC), generalized clonus (GC), and tonic-clonic (TC) seizures were summed to assign each mouse a seizure score that was used as a quantitative trait measure for mapping according to the following equation: Seizure score = $[(0.2)(1 / P C$ latency $)+(0.3)(1 / G C$ latency $)+(0.5)(1 / T C$ latency $)] \times 1000$. The weighting factors $(0.2,0.3$ and 0.5$)$ in the equation were included as means of incorporating a 


\section{Tantra et al REVISED MANUSCRIPT}

measure of the progressive nature of the PTZ-induced seizure phenotype into the severity rating because generalized clonus is regarded as a more significant event than partial clonus, and tonic hind limb extension is regarded as the most severe component of the phenotype. Therefore, the seizure score reflects the degree of progression of the seizure phenotype in each mouse.(Ferraro et al, 1999) The test was performed on adult Mecp2 TG of FVB/N and C57BL/6N background (30-40 weeks old). Male (FVB/N: 6 TG, 10 WT; C57BL/6N: 12 TG, 10 WT) and female mice (FVB/N: 14 TG, 14 WT; C57BL/6N: 10 TG, 11 WT) were tested.

\section{Human sample}

\section{Schizophrenic patients (discovery sample)}

The GRAS (Göttingen Research Association for Schizophrenia) data collection(Ribbe et al, 2010) was approved by the ethics committee of the Georg-August-University Göttingen (master committee) and respective review boards of collaborating centers. The project complies with the Helsinki Declaration. Patients fulfilling DSM-IV criteria for schizophrenia or schizoaffective disorder were included regardless of disease stage (acute, chronic, residual, or remitted). All study participants (European Caucasian 95.3\%; other 2.0\%; unknown $2.7 \%$ ) and, if applicable, their legal representatives gave written informed consent. Of the included 1052 patients, $68.2 \%$ were male $(\mathrm{N}=717)$ and $31.8 \%$ female $(\mathrm{N}=335)$. Average age was $39.14 \pm 12.56$ years (range 17 to 78 ).

\section{Healthy controls}

Healthy voluntary blood donors were recruited by the Department of Transfusion Medicine at the Georg-August-University of Göttingen according to national guidelines for blood donation. As such, they widely fulfill health criteria, ensured by a broad predonation screening process including standardized health questionnaires, interviews, and assessment of hemoglobin concentration, blood pressure, pulse, and body temperature. Of the $\mathrm{N}=1248$ successfully genotyped control subjects (European Caucasian $97.8 \%$; other $2 \%$; unknown $0.2 \%), 61.5 \%$ were male $(\mathrm{N}=768)$ and $38.5 \%$ female $(\mathrm{N}=480)$. Average age was $37.44 \pm 13.23$ years (range 18 to 69$)$.

Independent schizophrenia sample (replicate sample) 


\section{Tantra et al REVISED MANUSCRIPT}

To replicate in an independent sample the phenotype-genotype associations found in male schizophrenic GRAS patients, data from male schizophrenic subjects ( $N=385)$ of the Munich/Halle collection of Dan Rujescu could be analysed (Van den Oord et al, 2006). Also in this replicate sample, written informed consent had been obtained from all subjects after detailed and extensive description of the study, which was approved by the local ethics committee and carried out in accordance to the ethical standards laid down in the Declarations of Helsinki.

\section{Phenotyping - Target variables}

All schizophrenic patients of the GRAS data collection were comprehensively phenotyped (Ribbe et al, 2010). To prove our hypothesis that MECP2 genotypes modulate aggressive behavior in human subjects, we selected target variables closely related to (poor impulse control) or predicting (excitement) aggressive behavior in schizophrenic individuals (Arango et al, 1999; Colasanti et al, 2010; Soyka et al, 2007). To assess the severity of poor impulse control, item 14 of the positive and negative syndrome scale (PANSS) (Kay et al, 1987) was used ('disordered regulation and control of action on inner urges, resulting in sudden, unmodulated, arbitrary or misdirected discharge of tension and emotions without concern about consequences'). From the subscale covering positive symptomatology, item 4 assessing excitement ('hyperactivity as reflected in accelerated motor behavior, heightened responsivity to stimuli, hypervigilance or excessive mood lability') was employed (Tables2,3). Both PANSS readouts were also available in the independent sample of schizophrenic individuals (replicate sample). The choice of items is supported by the literature: Cheung and colleagues compared aggressive and non-aggressive schizophrenia patients (aggression assessed by the Staff Observation Aggression Scale) with respect to single items of the PANSS (Cheung et al, 1997). The largest group difference was found for the PANSS item 'poor impulse control'. Strikingly, the aggressive group had an average score of 4 (range: 1-7) on this item. Even after controlling for the total level of psychopathology, the associations of 'poor impulse control' and aggressive behavior remained significant. Additionally, in a more recent prospective study, 'poor impulse control' as measured by PANSS was highly predictive of aggressive behavior (assessed by the Overt Aggression Scale)(Nolan et al, 2005). 


\section{Tantra et al REVISED MANUSCRIPT}

\section{Phenotyping - Control variables}

Control variables (and potential confounders) are also presented in Tables2,3. Sociodemographic data (age, years of education, unemployment rate), a cognition composite score and clinical variables describing disease severity were used to characterize the GRAS sample and exclude potential confounding effects explaining the target phenotype-genotype associations. The cognition composite score(Begemann et al, 2011) represents the mean of 3 z-standardized neuropsychological measures of higher cognitive functioning: reasoning ability (Leistungsprüfsystem subtest 3 (Horn, 1983)), executive functioning (Trail-Making Test B (Reitan, 1958)) and verbal learning and memory (Verbal Learning and Memory Test (Helmstaedter et al, 2001)). As further clinical variables, the general, positive and negative scores of the PANSS (target variables excluded from respective scores), chlorpromazine equivalents (standardized dosage of antipsychotic medication) (Rijcken et al, 2003; Woods, 2003) and GAF (Global assessment of functioning (Wittchen et al, 1997)) as measure of impaired psychological, social and occupational performance were used.

\section{DNA extraction, normalization and genotyping}

Genomic DNA was purified from whole blood using JETQUICK Blood \& Cell Culture DNA Spin Kit (Genomed $\mathrm{GmbH}$, Löhne, Germany) according to the manufacturer's protocol. Resulting DNA samples were aliquoted and stored at $-80^{\circ} \mathrm{C}$. For further analysis, DNA was normalized to 50ng/ $\mu$ l with an automated robotic platform (Microlab Star, Hamilton, Bonaduz, Switzerland). For quality control, each sample was analyzed with a $0.8 \%$ agarose gel. Genotyping was performed using SimpleProbes (TIB Molbiol, Berlin, Germany) on LightCycler 480 (Roche Diagnostics, Basel, Switzerland), according to the manufacturer's instructions.

\section{Transfection studies}

\section{Cell lines}

Human embryonic kidney 293 (HEK293) and mouse neuroblastoma (N2a) cells were maintained in DMEM supplemented with $1 \mathrm{~g} / \mathrm{l}$ glucose, L-glutamine (Glutamax), $5 \%$ fetal calf serum, $100 \mathrm{U} / \mathrm{ml}$ penicilling sodium and $100 \mu \mathrm{g} / \mathrm{ml}$ streptomycin sulfate. For luciferase assays, cells were seeded into 12-well plates $(220,000$ cells/well/2ml medium), cultured for $24 \mathrm{~h}$ and co-transfected using Lipofectamine 2000 (Invitrogen, Karlsruhe, Germany), following the manufacturer's instructions. 


\section{Tantra et al REVISED MANUSCRIPT}

\section{Luciferase reporter constructs and detection assay}

The reporter plasmids phRL-TK rs2734647C, or rs2734647T, respectively, were constructed by cloning a 3'UTR fragment of 346 bp and including the SNP downstream of the Renilla luciferase open reading frame, making use of the Xbal restriction site of phRL-TK (forward primer: 5'-ATTATCTAGACCAGGTCTACCCCTCCCGGC-3', reverse primer: 5'-ATTATCTAGAGGCTGCTCCCTGTCCCAGGT-3'). Sequence integrity was verified using Sanger sequencing. Of the Renilla luciferase reporter construct (phRL-TK rs2734647C, or rs2734647T, respectively), $1 \mu \mathrm{g}$ (per well), plus $1 \mu \mathrm{g}$ (per well) of the reference construct pCMV-LacZ (Clontech, Mountain View, CA, USA) were cotransfected in the presence of 10pg of mirVana miRNA mimic hsa-miR-4711-3p, hsamiR-511, hsa-miR-515-3p, hsa-miR-519e-3p, negative control \#2 (all Life Technologies, Darmstadt, Germany), or no miRNA, respectively. After 24h, cells were split in 96 well plates, creating four technical replicates for each condition, and separately for luciferase and beta-galactosidase measurement. Enzyme activity was determined using a Mithras LB 940 Plate Reader (Berthold, Bad Wildbad, Germany). Renilla luciferase activity was normalized to beta-galactosidase activity.

\section{RNA isolation and quantitative PCR}

Peripheral blood mononuclear cells (PBMC) were isolated from citrate blood of men with rs2734647C or rs2734647T genotype (X-chromosomal gene), applying a standard isolation procedure (Ficoll-Paque Plus, GEHealthcare, München, Germany). Human RNA was extracted from deep-frozen human brain samples of adult male subjects who had been free of neuropsychiatric diseases, from placenta or PBMC, respectively, using a miRNeasy Mini kit (Qiagen, Hilden, Germany). The same kit was employed for isolation of mouse RNA from whole E17 embryo (divided into body and head), hippocampus, amygdala, placenta, and cultured microglia. Synthesis of cDNA was done by the SuperScriptIII system (Invitrogen, Karlsruhe, Germany). Detection of MECP2 cDNA was performed using SYBR green (Roche, Diagnostics $\mathrm{GmbH}$, Mannheim, Germany) and specific primer pairs amplifying the MECP2_e2 isoform (NCBI reference sequence NM_004992), spanning exons 2-3 (forward primer: 5'CAGCTCCAACAGGATTCCAT-3', reverse primer: 5'- TGGAGGTCCTGGTCTTCTGA3'), or both isoforms (NM_004992 and NM_001110792), spanning exons 3-4 (forward primer: 5'- AGCTTAAGCAAAGGAAATCTGG-3', reverse primer: 5'- 


\section{Tantra et al REVISED MANUSCRIPT}

GCTTTTCCCTGGGGATTG-3'). Specific Taqman microRNA assays were used to detect hsa-miR-511, hsa-miR-4711-3p, or mmu-miR-511 (Applied Biosystems, Foster City, CA, USA), following the manufacturer's instructions. MECP2 expression levels were normalized to GAPDH, human miRNA expression levels to RNU43, and mouse miRNA expression levels to sno-142.

\section{Statistics}

All experimental data acquisition was done by experimenters unaware of group assignment ('blinded'). Mouse behavioral data were analyzed by Mann-Whitney $U$ test or 2-way analysis of variance including posthoc Bonferroni testing, where applicable, using Prism4 (GraphPad Software, San Diego, CA, USA). All data are presented as mean $\pm S E M$, unless stated otherwise. Luciferase assay results from each experiment were normalized to the relative luciferase activity without miRNA co-transfection and analyzed using one-tailed t-tests. qPCR results were normalized to the respective control genes and analyzed using one-tailed t-tests. PLINK (v1.07) (Purcell et al, 2007) was used for the analysis of statistical association between single SNPs and case or control status (allelic test), and to test for deviations from Hardy-Weinberg equilibrium. Statistical analyses of phenotype-genotype associations in the human samples (both GRAS and replicate sample) were performed using SPSS for Windows version 17.0 (SPSS Inc., Chicago, IL, USA; http://www.spss.com). As MECP2 is X-linked, analyses were performed separately for men and women. Genotype differences with respect to the target variables were assessed by analysis of covariance. Covariates age at examination, years of education, chlorpromazine equivalents and severity of negative symptoms (PANSS) were used, as these parameters are likely to influence the extent to which impulsive aggressive behavior becomes obvious in a social situation. For the cognition composite score, analysis of covariance with covariates age, chlorpromazine equivalents and severity of negative symptoms was applied as these variables have been widely reported to confound performance on neuropsychological tests(Bilder et al, 1992; Hori et al, 2006). Genotype differences with respect to sociodemographic and clinical measures were tested non-parametrically using $\chi^{2}$ (nominal variables) or MannWhitney-U/Kruskal-Wallis tests (continuous variables). All $\mathrm{p}$-values derived from statistical models for the GRAS sample are two-sided (Tables2,3). For the replicate sample one-sided $p$-values are displayed (Table4). Nominal significance level for all analyses was set to $p<0.05$. 


\section{Tantra et al REVISED MANUSCRIPT}

\section{ACKNOWLEDGEMENTS:}

We are indebted to all patients for their participation in the GRAS (Göttingen Research Association for Schizophrenia) study, and all collaborating GRAS centers for their support. We are grateful to all colleagues who contributed to the GRAS data collection. We would also like to thank Anja Ronnenberg and Maria Zerche for excellent technical assistance and Dr. Wilko Hinrichs for his help regarding luciferase assay reading.

\section{AUTHOR CONTRIBUTIONS:}

Conceived and designed the study: $\mathrm{HE}, \mathrm{MT}, \mathrm{CH}, \mathrm{AK}$.

Prepared the data: MT, CH, AK, MB, LD, CB, KH, BS, ACV, BE, IG, AT, BK, AH, WSS. Analyzed the data: MT, CH, AK, LD, KH, DR, AUM.

Wrote the paper: $\mathrm{HE}, \mathrm{MT}, \mathrm{AK}, \mathrm{CH}$.

Critical review and approval of the manuscript: ALL AUTHORS.

DISCLOSURE: The authors declare that they have no competing interests.

\section{FUNDING:}

This work was supported by the Max Planck Society, the Max-Planck-Förderstiftung, the DFG Center for Nanoscale Microscopy \& Molecular Physiology of the Brain (CNMPBB2) and COST action ECMNet. Christian Hammer acknowledges grant support by the Daimler \& Benz Foundation as well as by the Brain \& Behavior Foundation. 


\section{Tantra et al REVISED MANUSCRIPT}

\section{The paper explained}

\section{Problem}

The transcriptional regulator methyl-CpG-binding protein2, MECP2, is renowned because of the devastating neurodevelopmental disorder Rett syndrome, caused by partial or complete loss of its function. The very same gene, when duplicated, induces a similar disorder, indicating the necessity of tight MECP2 regulation. Among the vast array of other genes influenced by MECP2, many are involved in modulating behavior. Surprisingly, nothing is known on the physiological (i.e. non disease-related) effects on behavior of subtle MECP2 expression differences.

\section{Results}

We present here a translational study, reaching from mouse to man to cell, that explores behavioral consequences of mildly increased MECP2 expression across species. In both man and mouse, we find that the behavioral target domain directed by MECP2 is male social aggression. This in turn is subject to modification by the genetic background, as we demonstrate by comparing an inherent aggressive with a less aggressive mouse strain. Likewise, we identify in two independent cohorts of schizophrenic individuals, i.e. a discovery and a replicate sample, a role of MECP2 in aggressive human behavior and show that a polymorphism in the untranslated region of the gene determines binding efficiency of another brain-expressed regulator, microRNA511. Notably, the genotype-dependent expression difference of 40-50\% (rs2734647$\mathrm{C}>\mathrm{T}$ ) in man, at least partially mediated by microRNA-511, is comparable to the level of transgenic overexpression in our mice, emphasizing the physiological significance of these findings.

\section{Impact}

Genetic regulation of behavior is still poorly understood. We present here a translational study, extending from mouse to man to cell, that explores for the first time behavioral consequences of mildly ( $50 \%)$ increased MECP2 expression across species. This is achieved by transgenic Mecp2 overexpression in mice and through genetic variationinduced non-regulability of MECP2 gene expression (rs 2734647-C: no miR-511-mediated MECP2 downregulation) in humans. We find MECP2 to be a modulator of social aggression in mouse and man. While the mouse data shows an interaction between genetic background and Mecp2 expression on behavior, the human data provides means by which genetic variation may affect MECP2 expression and result in behavioral change. 


\section{Tantra et al REVISED MANUSCRIPT}

\section{REFERENCES}

Albert FW, Somel M, Carneiro M, Aximu-Petri A, Halbwax M, Thalmann O, Blanco-Aguiar JA, Plyusnina IZ, Trut L, Villafuerte $R$ et al (2012) A comparison of brain gene expression levels in domesticated and wild animals. PLoS Genet 8: e1002962

Amir RE, Van den Veyver IB, Wan M, Tran CQ, Francke U, Zoghbi HY (1999) Rett syndrome is caused by mutations in X-linked MECP2, encoding methyl-CpG-binding protein 2. Nat Genet 23: $185-188$

Arango C, Calcedo Barba A, Gonzalez S, Calcedo Ordonez A (1999) Violence in inpatients with schizophrenia: a prospective study. Schizophr Bull 25: 493-503

Bauman MD, Toscano JE, Mason WA, Lavenex P, Amaral DG (2006) The expression of social dominance following neonatal lesions of the amygdala or hippocampus in rhesus monkeys (Macaca mulatta). Behav Neurosci 120: 749-760

Begemann M, Grube S, Papiol S, Malzahn D, Krampe H, Ribbe K, Friedrichs H, Radyushkin KA, El-Kordi A, Benseler $F$ et al (2011) Modification of cognitive performance in schizophrenia by complexin 2 gene polymorphisms. Arch Gen Psychiatry 67: 879-888

Ben-Shachar S, Chahrour M, Thaller C, Shaw CA, Zoghbi HY (2009) Mouse models of MeCP2 disorders share gene expression changes in the cerebellum and hypothalamus. Hum Mol Genet 18: 2431-2442

Berlin HA, Rolls ET, Kischka U (2004) Impulsivity, time perception, emotion and reinforcement sensitivity in patients with orbitofrontal cortex lesions. Brain 127: 1108-1126

Bienvenu T, Chelly J (2006) Molecular genetics of Rett syndrome: when DNA methylation goes unrecognized. Nat Rev Genet 7: 415-426

Bilder RM, Turkel E, Lipschutz-Broch L, Lieberman JA (1992) Antipsychotic medication effects on neuropsychological functions. Psychopharmacol Bull 28: 353-366

Bird A (2008) The methyl-CpG-binding protein MeCP2 and neurological disease. Biochem Soc Trans 36: 575-583

Bodda C, Tantra M, Mollajew R, Arunachalam JP, Can K, Rosenberger A, Mironov SL, Ehrenreich H, Mannan AU (2013) Mild Overexpression of Mecp2 in Mice Causes a Higher Susceptibility toward Seizures. Am J Pathol 183: 195-210

Brower MC, Price BH (2001) Neuropsychiatry of frontal lobe dysfunction in violent and criminal behaviour: a critical review. J Neurol Neurosurg Psychiatry 71: 720-726

Chahrour M, Jung SY, Shaw C, Zhou X, Wong ST, Qin J, Zoghbi HY (2008) MeCP2, a key contributor to neurological disease, activates and represses transcription. Science 320: 12241229 


\section{Tantra et al REVISED MANUSCRIPT}

Chahrour M, Zoghbi HY (2007) The story of Rett syndrome: from clinic to neurobiology. Neuron 56: 422-437

Chao HT, Chen H, Samaco RC, Xue M, Chahrour M, Yoo J, Neul JL, Gong S, Lu HC, Heintz N et al (2010) Dysfunction in GABA signalling mediates autism-like stereotypies and Rett syndrome phenotypes. Nature 468: 263-269

Chao HT, Zoghbi HY (2012) MeCP2: only 100\% will do. Nat Neurosci 15: 176-177

Chen RZ, Akbarian S, Tudor M, Jaenisch R (2001) Deficiency of methyl-CpG binding protein-2 in CNS neurons results in a Rett-like phenotype in mice. Nat Genet 27: 327-331

Chen WG, Chang Q, Lin Y, Meissner A, West AE, Griffith EC, Jaenisch R, Greenberg ME (2003) Derepression of BDNF transcription involves calcium-dependent phosphorylation of MeCP2. Science 302: 885-889

Cheung P, Schweitzer I, Crowley K, Tuckwell V (1997) Aggressive behaviour in schizophrenia: the role of psychopathology. Aust N Z J Psychiatry 31: 62-67

Cohen S, Zhou Z, Greenberg ME (2008) Medicine. Activating a repressor. Science 320: 11721173

Colasanti A, Paletta S, Moliterno D, Mazzocchi A, Mauri MC, Altamura AC (2010) Symptom dimensions as predictors of clinical outcome, duration of hospitalization, and aggressive behaviours in acutely hospitalized patients with psychotic exacerbation. Clin Pract Epidemiol Ment Health 6: 72-78

Collins AL, Levenson JM, Vilaythong AP, Richman R, Armstrong DL, Noebels JL, David Sweatt J, Zoghbi HY (2004) Mild overexpression of MeCP2 causes a progressive neurological disorder in mice. Hum Mol Genet 13: 2679-2689

Coy JF, Sedlacek Z, Bachner D, Delius H, Poustka A (1999) A complex pattern of evolutionary conservation and alternative polyadenylation within the long 3 "-untranslated region of the methyl-CpG-binding protein 2 gene (MeCP2) suggests a regulatory role in gene expression. Hum Mol Genet 8: 1253-1262

Deacon RM (2006) Assessing nest building in mice. Nat Protoc 1: 1117-1119

El-Kordi A, Winkler D, Hammerschmidt K, Kastner A, Krueger D, Ronnenberg A, Ritter C, Jatho $\mathrm{J}$, Radyushkin K, Bourgeron T et al (2012) Development of an autism severity score for mice using Nlgn4 null mutants as a construct-valid model of heritable monogenic autism. Behav Brain Res 251: 41-49

Ferraro TN, Golden GT, Smith GG, St Jean P, Schork NJ, Mulholland N, Ballas C, Schill J, Buono RJ, Berrettini WH (1999) Mapping loci for pentylenetetrazol-induced seizure susceptibility in mice. J Neurosci 19: 6733-6739 


\section{Tantra et al REVISED MANUSCRIPT}

Fyffe SL, Neul JL, Samaco RC, Chao HT, Ben-Shachar S, Moretti P, McGill BE, Goulding EH, Sullivan E, Tecott LH et al (2008) Deletion of Mecp2 in Sim1-expressing neurons reveals a critical role for MeCP2 in feeding behavior, aggression, and the response to stress. Neuron 59: 947-958

Garfield AS, Cowley M, Smith FM, Moorwood K, Stewart-Cox JE, Gilroy K, Baker S, Xia J, Dalley JW, Hurst LD et al (2011) Distinct physiological and behavioural functions for parental alleles of imprinted Grb10. Nature 469: 534-538

Gaskill BN, Gordon CJ, Pajor EA, Lucas JR, Davis JK, Garner JP (2012) Heat or insulation: behavioral titration of mouse preference for warmth or access to a nest. PLoS One 7: e32799

Gopisetty G, Xu J, Sampath D, Colman H, Puduvalli VK (2012) Epigenetic regulation of CD133/PROM1 expression in glioma stem cells by Sp1/myc and promoter methylation. Oncogene 32: 3119-3129

Greenberg G (1972) The effects of ambient temperature and population density on aggression in two inbred strains of mice, Mus musculus. Behaviour 42: 119-130

Grimson A, Farh KK, Johnston WK, Garrett-Engele P, Lim LP, Bartel DP (2007) MicroRNA targeting specificity in mammals: determinants beyond seed pairing. Mol Cell 27: 91-105

Guy J, Hendrich B, Holmes M, Martin JE, Bird A (2001) A mouse Mecp2-null mutation causes neurological symptoms that mimic Rett syndrome. Nat Genet 27: 322-326

Haldane M, Cunningham G, Androutsos C, Frangou S (2008) Structural brain correlates of response inhibition in Bipolar Disorder I. J Psychopharmacol 22: 138-143

Hammerschmidt K, Radyushkin K, Ehrenreich H, Fischer J (2012) The structure and usage of female and male mouse ultrasonic vocalizations reveal only minor differences. PLoS One 7: e41133

Helmstaedter C, Lendt M, Lux S (2001) Verbal Learning and Memory Test (VLMT), Goettingen, Germany: Beltz

Hori H, Noguchi H, Hashimoto R, Nakabayashi T, Omori M, Takahashi S, Tsukue R, Anami K, Hirabayashi N, Harada S et al (2006) Antipsychotic medication and cognitive function in schizophrenia. Schizophr Res 86: 138-146

Horn NR, Dolan M, Elliott R, Deakin JF, Woodruff PW (2003) Response inhibition and impulsivity: an fMRI study. Neuropsychologia 41: 1959-1966

Horn W (1983) Leistungsprüfsystem (LPS), Goettingen, Germany: Hogrefe

Huppke P, Maier EM, Warnke A, Brendel C, Laccone F, Gartner J (2006) Very mild cases of Rett syndrome with skewed $X$ inactivation. J Med Genet 43: 814-816 


\section{Tantra et al REVISED MANUSCRIPT}

Ito W, Chehab M, Thakur S, Li J, Morozov A (2011) BDNF-restricted knockout mice as an animal model for aggression. Genes Brain Behav 10: 365-374

Jamain S, Radyushkin K, Hammerschmidt K, Granon S, Boretius S, Varoqueaux F, Ramanantsoa N, Gallego J, Ronnenberg A, Winter D et al (2008) Reduced social interaction and ultrasonic communication in a mouse model of monogenic heritable autism. Proc Natl Acad Sci U S A 105: 1710-1715

Joyner AH, J CR, Bloss CS, Bakken TE, Rimol LM, Melle I, Agartz I, Djurovic S, Topol EJ, Schork NJ et al (2009) A common MECP2 haplotype associates with reduced cortical surface area in humans in two independent populations. Proc Natl Acad Sci U S A 106: 15483-15488

Kay SR, Fiszbein A, Opler LA (1987) The positive and negative syndrome scale (PANSS) for schizophrenia. Schizophr Bull 13: 261-276

Kerr B, Alvarez-Saavedra M, Saez MA, Saona A, Young JI (2008) Defective body-weight regulation, motor control and abnormal social interactions in Mecp2 hypomorphic mice. Hum Mol Genet 17: 1707-1717

Kertesz M, lovino N, Unnerstall U, Gaul U, Segal E (2007) The role of site accessibility in microRNA target recognition. Nat Genet 39: 1278-1284

Kifayathullah LA, Arunachalam JP, Bodda C, Agbemenyah HY, Laccone FA, Mannan AU (2010) MeCP2 Mutant Protein Is Expressed in Astrocytes as well as in Neurons and Localizes in the Nucleus. Cytogenet Genome Res 129: 290-297

Kuc KA, Gregersen BM, Gannon KS, Dodart JC (2006) Holeboard discrimination learning in mice. Genes Brain Behav 5: 355-363

Kurian JR, Bychowski ME, Forbes-Lorman RM, Auger CJ, Auger AP (2008) Mecp2 organizes juvenile social behavior in a sex-specific manner. J Neurosci 28: 7137-7142

Kurian JR, Forbes-Lorman RM, Auger AP (2007) Sex difference in mecp2 expression during a critical period of rat brain development. Epigenetics 2: 173-178

Lewis BP, Burge CB, Bartel DP (2005) Conserved seed pairing, often flanked by adenosines, indicates that thousands of human genes are microRNA targets. Cell 120: 15-20

Mandillo S, Tucci V, Holter SM, Meziane H, Banchaabouchi MA, Kallnik M, Lad HV, Nolan PM, Ouagazzal AM, Coghill EL et al (2008) Reliability, robustness, and reproducibility in mouse behavioral phenotyping: a cross-laboratory study. Physiol Genomics 34: 243-255

Martinowich K, Hattori D, Wu H, Fouse S, He F, Hu Y, Fan G, Sun YE (2003) DNA methylationrelated chromatin remodeling in activity-dependent BDNF gene regulation. Science 302: 890893

Messeri P, Eleftheriou BE, Oliverio A (1975) Dominance behavior: A phylogenetic analysis in the mouse. Physiol Behav 14: 53-58 


\section{Tantra et al REVISED MANUSCRIPT}

Mineur YS, Crusio WE (2002) Behavioral and neuroanatomical characterization of FVB/N inbred mice. Brain Res Bull 57: 41-47

Moretti P, Bouwknecht JA, Teague R, Paylor R, Zoghbi HY (2005) Abnormalities of social interactions and home-cage behavior in a mouse model of Rett syndrome. Hum Mol Genet 14: $205-220$

Moy SS, Nadler JJ, Young NB, Nonneman RJ, Grossman AW, Murphy DL, D'Ercole AJ, Crawley JN, Magnuson TR, Lauder JM (2009) Social approach in genetically engineered mouse lines relevant to autism. Genes Brain Behav 8: 129-142

Nolan KA, Volavka J, Czobor P, Sheitman B, Lindenmayer JP, Citrome LL, McEvoy J, Lieberman JA (2005) Aggression and psychopathology in treatment-resistant inpatients with schizophrenia and schizoaffective disorder. J Psychiatr Res 39: 109-115

O'Leary T, Gunn R, Brown R (2013) What are We Measuring When We Test Strain Differences in Anxiety in Mice? Behav Genet 43: 34-50

Paylor R, Crawley JN (1997) Inbred strain differences in prepulse inhibition of the mouse startle response. Psychopharmacology 132: 169-180

Pearson BL, Defensor EB, Pobbe RLH, Yamamoto LHL, Bolivar VJ, Blanchard DC, Blanchard RJ (2012) Mecp2 Truncation in Male Mice Promotes Affiliative Social Behavior. Behav Genet 42: 299-312

Peters SU, Hundley RJ, Wilson AK, Warren Z, Vehorn A, Carvalho CM, Lupski JR, Ramocki MB (2013) The behavioral phenotype in MECP2 duplication syndrome: a comparison with idiopathic autism. Autism Res 6: 42-50

Pietropaolo S, Guilleminot A, Martin B, D'Amato FR, Crusio WE (2011) Genetic-background modulation of core and variable autistic-like symptoms in Fmr1 knock-out mice. PLoS One 6: e17073

Pugh PL, Ahmed SF, Smith MI, Upton N, Hunter AJ (2004) A behavioural characterisation of the FVB/N mouse strain. Behav Brain Res 155: 283-289

Purcell S, Neale B, Todd-Brown K, Thomas L, Ferreira MA, Bender D, Maller J, Sklar P, de Bakker PI, Daly MJ et al (2007) PLINK: a tool set for whole-genome association and population-based linkage analyses. Am J Hum Genet 81: 559-575

Ramocki MB, Peters SU, Tavyev YJ, Zhang F, Carvalho CM, Schaaf CP, Richman R, Fang P, Glaze DG, Lupski JR et al (2009) Autism and other neuropsychiatric symptoms are prevalent in individuals with MeCP2 duplication syndrome. Ann Neurol 66: 771-782

Ramocki MB, Tavyev YJ, Peters SU (2010) The MECP2 duplication syndrome. Am J Med Genet A 152A: 1079-1088 


\section{Tantra et al REVISED MANUSCRIPT}

Reitan RM (1958) Validity of the Trail Making Test as an indicator of organic brain damage. Perceptual and Motor Skills 8: 271-276

Ribbe K, Friedrichs H, Begemann M, Grube S, Papiol S, Kästner A, Gerchen MF, Ackermann V, Tarami A, Treitz A et al (2010) The cross-sectional GRAS sample: a comprehensive phenotypical data collection of schizophrenic patients. BMC Psychiatry 10: 91

Rijcken CA, Monster TB, Brouwers JR, de Jong-van den Berg LT (2003) Chlorpromazine equivalents versus defined daily doses: how to compare antipsychotic drug doses? J Clin Psychopharmacol 23: 657-659

Samaco RC, Fryer JD, Ren J, Fyffe S, Chao HT, Sun Y, Greer JJ, Zoghbi HY, Neul JL (2008) A partial loss of function allele of methyl-CpG-binding protein 2 predicts a human neurodevelopmental syndrome. Hum Mol Genet 17: 1718-1727

Samaco RC, Mandel-Brehm C, Chao H-T, Ward CS, Fyffe-Maricich SL, Ren J, Hyland K, Thaller C, Maricich SM, Humphreys P et al (2009) Loss of MeCP2 in aminergic neurons causes cellautonomous defects in neurotransmitter synthesis and specific behavioral abnormalities. Proc Natl Acad Sci U S A 106: 21966-21971

Samaco RC, Mandel-Brehm C, McGraw CM, Shaw CA, McGill BE, Zoghbi HY (2012) Crh and Oprm1 mediate anxiety-related behavior and social approach in a mouse model of MECP2 duplication syndrome. Nat Genet 44: 206-211

Samaco RC, McGraw CM, Ward CS, Sun Y, Neul JL, Zoghbi HY (2013) Female Mecp2(+/-) mice display robust behavioral deficits on two different genetic backgrounds providing a framework for pre-clinical studies. Hum Mol Genet 22: 96-109

Satoh Y, Endo S, Nakata T, Kobayashi Y, Yamada K, Ikeda T, Takeuchi A, Hiramoto T, Watanabe Y, Kazama T (2011) ERK2 contributes to the control of social behaviors in mice. J Neurosci 31: 11953-11967

Shahbazian M, Young J, Yuva-Paylor L, Spencer C, Antalffy B, Noebels J, Armstrong D, Paylor R, Zoghbi H (2002) Mice with truncated MeCP2 recapitulate many Rett syndrome features and display hyperacetylation of histone H3. Neuron 35: 243-254

Siever L (2008) Neurobiology of aggression and violence. Am J Psychiatry 165: 429-442

Soyka M, Graz C, Bottlender R, Dirschedl P, Schoech H (2007) Clinical correlates of later violence and criminal offences in schizophrenia. Schizophr Res 94: 89-98

Sun YE, Wu H (2006) The ups and downs of BDNF in Rett syndrome. Neuron 49: 321-323

Tserel L, Runnel T, Kisand K, Pihlap M, Bakhoff L, Kolde R, Peterson H, Vilo J, Peterson P, Rebane A (2011) MicroRNA expression profiles of human blood monocyte-derived dendritic cells and macrophages reveal miR-511 as putative positive regulator of Toll-like receptor $4 . \mathrm{J}$ Biol Chem 286: 26487-26495 


\section{Tantra et al REVISED MANUSCRIPT}

Van den Oord EJ, Rujescu D, Robles JR, Giegling I, Birrell C, Bukszar J, Murrelle L, Moller HJ, Middleton L, Muglia P (2006) Factor structure and external validity of the PANSS revisited. Schizophr Res 82: 213-223

Whelan R, Conrod PJ, Poline JB, Lourdusamy A, Banaschewski T, Barker GJ, Bellgrove MA, Buchel C, Byrne M, Cummins TD et al (2012) Adolescent impulsivity phenotypes characterized by distinct brain networks. Nat Neurosci 15: 920-925

Wittchen H-U, Zaudig M, Fydrich T (1997) SCID-I: Structured Clinical Interview for DSM-IV Disorders, Goettingen, Germany: Hogrefe

Wojcik SM, Tantra M, Stepniak B, Man KN, Muller-Ribbe K, Begemann M, Ju A, Papiol S, Ronnenberg A, Gurvich A et al (2013) Genetic Markers of a Munc13 Protein Family Member, BAIAP3, Are Gender-Specifically Associated with Anxiety and Benzodiazepine Abuse in Mouse and Man. Mol Med 19: 135-148

Wolfer D, Lipp H (2000) Dissecting the behaviour of transgenic mice: is it the mutation, the genetic background, or the environment? Exp Physiol 85: 627-634

Wong EH, So HC, Li M, Wang Q, Butler AW, Paul B, Wu HM, Hui TC, Choi SC, So MT et al (2013) Common Variants on Xq28 Conferring Risk of Schizophrenia in Han Chinese. Schizophr Bull [Epub ahead of print]

Woods SW (2003) Chlorpromazine equivalent doses for the newer atypical antipsychotics. J Clin Psychiatry 64: 663-667

Wu H, Tao J, Chen PJ, Shahab A, Ge W, Hart RP, Ruan X, Ruan Y, Sun YE (2010) Genome-wide analysis reveals methyl-CpG-binding protein 2-dependent regulation of microRNAs in a mouse model of Rett syndrome. Proc Natl Acad Sci U S A 107: 18161-18166

Zetzsche T, Preuss UW, Frodl T, Schmitt G, Seifert D, Munchhausen E, Tabrizi S, Leinsinger G, Born C, Reiser $M$ et al (2007) Hippocampal volume reduction and history of aggressive behaviour in patients with borderline personality disorder. Psychiatry Res 154: 157-170 


\section{Tantra et al REVISED MANUSCRIPT}

\section{FIGURE LEGENDS:}

Figure 1: Spontaneous home cage activity of FVB/N and C57BL/6N mice of both genders is modulated by mild Mecp2 overexpression. Results for male and female mice of both strains are presented. With the exception of male FVB/N mice, locomotion, immobility and climbing reveal similar TG effects across genders and genetic backgrounds. $\mathrm{N}=10-28$; mean \pm SEM given .

Figure 2: Territorial aggressive behavior in male mice is influenced by Mecp2 overexpression and genetic background. A. Latency to attack in the resident-intruder test is significantly reduced in male FVB/N TG mice but increased in C57BL/6N. Note the different cut-off for the 2 strains. B. Sociability testing in the tripartite chamber reveals a highly significant preference of male mice, independent of the genetic background, for a stranger mouse as compared to an object (empty cage). $\mathrm{N}=10-24$; mean \pm SEM given.

Figure 3: Male TG C57BL/6N mice show reduced territorial social interaction as well as inferior social competence. Upper 2 rows: Frequency and duration of determinants of territorial social interaction, i.e. follow/chase behavior, nose/snout sniff and anogenital sniff, are consistently reduced in TG carriers. Lower row: TG mice are inferior in nest building, social dominance behavior and ultrasound vocalization. $\mathrm{N}=10$ 23; mean \pm SEM given.

Figure 4: Pentylenetetrazole-induced seizure propensity is increased upon mild Mecp2 overexpression independent of strain and gender, whereas the startle response is augmented in females only. Upper row: Higher seizure scores are found in TG carriers across gender and genetic backgrounds. N=6-14; Lower row: Significant increase in the startle response is observed only in female mice of both genetic backgrounds. $\mathrm{N}=11-28$; mean \pm SEM given. 


\section{Tantra et al REVISED MANUSCRIPT}

Figure 5: Basic genetics of MECP2: Gene structure, Hardy-Weinberg statistics, linkage disequilibrium, and case-control analysis in schizophrenic (GRAS) patients and healthy individuals. A. Schematic overview of MECP2 isoforms e1 and e2, including SNP positions. Digits depict exon numbers, solid black lines exon usage for the respective isoform. Dashed lines denote SNP positions. Black fillings in boxes denote coding sequence, isoform-specific in exons 1 and 2. B. Test for deviation from Hardy-Weinberg-Equilibrium (HWE) in females only due to X-chromosomal location of MECP2. C. Case-control association analysis - separate for both genders - reveals similar distribution of SNPs in patients and controls. D. Linkage disequilibrium for all included GRAS patients and controls.

Figure 6: SNP rs2734647 in the 3'UTR of MECP2: Search for mechanistic insight

A. Human miRNAs predicted to bind to the MECP2 3'UTR in an rs2734647 allelespecific manner. The bases corresponding to the SNP position are black-shadowed. Numbers left and right of the nucleotide sequence refer to its base-pair position within the miRNA sequence. $\mathbf{B}, \mathbf{C}$. Luciferase assay results showing relative luciferase activity in HEK293 and N2a cells after co-transfection of candidate miRNAs with phRL-TK rs2734647C, or rs2734647T, respectively; mean \pm SEM; N=7 for all conditions. Statistical significance was calculated relative to the non-transfection control $(100 \%)$ D, E. Relative expression of hsa-miR-511 and of MECP2 isoform 2 (MECP2_e2) or both isoforms (MECP2_e1\&2) in aggression/impulsivity - relevant brain areas: $\mathrm{FC}=$ frontal cortex, $\mathrm{PFC}=$ prefrontal cortex, $\mathrm{TC}=$ temporal cortex, $\mathrm{OC}=$ occipital cortex, $\mathrm{HC}=$ hippocampus, $\mathrm{AM}=$ amygdala, as well as in placenta $(\mathrm{N}=1)$ as control tissue. Numbers of individual brains included in the analysis are given in brackets; mean \pm SEM. F. Relative expression of $M E C P 2 \_e 2$ or MECP2_e1\&2 in peripheral blood mononuclear cells (PBMC) of male patients dependent on rs2734647 genotype; $\mathrm{N}$ numbers of individuals in brackets; mean \pm SEM. G. Alignment of human and mouse MECP2 3‘UTRs around rs2734647 SNP position (black-shadowed) and human and mouse miR-511, illustrating a perfect species-specific seed match; hsa-miR-511 perfectly matches the human MECP2 3'UTR in case of rs2734647 T. Additional mismatches are gray-shadowed. 


\begin{tabular}{|c|c|c|c|c|c|c|c|c|c|c|c|c|c|c|c|c|c|c|c|c|c|}
\hline \multirow{3}{*}{$\begin{array}{l}\text { Behavioral paradigms } \\
\text { (order according to domains) }\end{array}$} & \multicolumn{7}{|c|}{ Cohort 1} & \multicolumn{7}{|c|}{ Cohort 2} & \multicolumn{7}{|c|}{ Cohort 3} \\
\hline & \multirow[b]{2}{*}{$\mathrm{Age}^{\mathrm{a}}$} & \multicolumn{2}{|c|}{ Male } & \multicolumn{3}{|c|}{ Female } & \multirow[b]{2}{*}{$\mathbf{P}$} & \multirow[b]{2}{*}{$\mathrm{Age}^{\mathrm{a}}$} & \multicolumn{2}{|c|}{ Male } & \multicolumn{3}{|c|}{ Female } & \multirow[b]{2}{*}{$\mathbf{P}$} & \multirow[b]{2}{*}{$\mathrm{Age}^{\mathrm{a}}$} & \multicolumn{2}{|c|}{ Male } & \multicolumn{4}{|c|}{ Female } \\
\hline & & $\begin{array}{c}\text { WT } \\
(\mathrm{N}=14)\end{array}$ & $\begin{array}{c}\mathrm{TG} \\
(\mathrm{N}=18)\end{array}$ & $\mathbf{P}$ & $\begin{array}{c}\text { WT } \\
(N=9)\end{array}$ & $\begin{array}{c}\mathrm{TG} \\
(\mathrm{N}=4)\end{array}$ & & & $\begin{array}{c}W T \\
(N=10-27)\end{array}$ & $\begin{array}{c}\mathrm{TG} \\
(\mathrm{N}=6-22)\end{array}$ & $\mathbf{P}$ & $\begin{array}{c}W T \\
(N=15-27)\end{array}$ & $\begin{array}{c}\mathrm{TG} \\
(\mathrm{N}=14-37)\end{array}$ & & & $\begin{array}{c}W T \\
(N=10-28)\end{array}$ & $\begin{array}{c}\mathrm{TG} \\
(\mathrm{N}=10-12)\end{array}$ & $\mathbf{P}$ & $\begin{array}{c}W T \\
(N=10-14)\end{array}$ & $\begin{array}{c}\mathrm{TG} \\
(\mathrm{N}=10-21)\end{array}$ & $\mathbf{P}$ \\
\hline \multicolumn{22}{|l|}{ Anxiety-like behavior } \\
\hline $\begin{array}{l}\text { Elevated plus maze (time in open } \\
\text { versus closed arms [s]) }\end{array}$ & 4 & & & & \multicolumn{3}{|c|}{ Genotype: $p=0.2, F=1.825$} & 12 & \multicolumn{3}{|c|}{ Genotype: $p=0.3, F=1.120$} & \multicolumn{3}{|c|}{ Genotype: $\mathrm{p}=0.8, \mathrm{~F}=0.082$} & 5 & \multicolumn{3}{|c|}{ Genotype: $p=0.4, F=0.567$} & \multicolumn{3}{|c|}{ Genotype: $p=0.6, F=0.342$} \\
\hline Activity & & & & & & & & & & & & & & & & & & & & & \\
\hline Open field (total distance [m]) & 4 & $73 \pm 6$ & $80 \pm 5.6$ & n.s. & $74 \pm 10$ & $85 \pm 3$ & n.s. & 12 & $78 \pm 6.3$ & $69 \pm 4.3$ & n.s. & $72 \pm 5.6$ & $71 \pm 3$ & n.s. & 5 & $87 \pm 2.8$ & $84 \pm 3.9$ & n.s. & $94 \pm 3.7$ & $89 \pm 3.4$ & n.s. \\
\hline Exploratory behavior & & & & & & & & & & & & & & & & & & & & & \\
\hline Hole board (holes visited $[\#]$ ) & & & & & & & & 12 & $12 \pm 1.4$ & $10 \pm 1.4$ & n.s. & $23 \pm 2.6$ & $21 \pm 1.7$ & n.s. & 6 & $15 \pm 1.2$ & $15 \pm 1.7$ & n.s. & $19 \pm 2.8$ & $21 \pm 2.2$ & n.s. \\
\hline Impulsivity & & & & & & & & & & & & & & & & & & & & & \\
\hline $\begin{array}{l}\text { Marble burying and digging } \\
\text { (marbles buried [\#]) }\end{array}$ & & & & & & & & 14 & $3.9 \pm 0.5$ & $4.6 \pm 0.6$ & n.s. & & & & $\begin{array}{c}7 \\
16\end{array}$ & $\begin{array}{c}4.5 \pm 0.5 \\
4.5 \pm 1\end{array}$ & $\begin{array}{c}4.6 \pm 0.7 \\
3.6 \pm 1\end{array}$ & $\begin{array}{l}\text { n.s. } \\
\text { n.s. }\end{array}$ & $\begin{array}{l}3.8 \pm 0.9 \\
1.5 \pm 0.7\end{array}$ & $\begin{array}{l}4.3 \pm 0.7 \\
3.5 \pm 1.0\end{array}$ & $\begin{array}{l}\text { n.s. } \\
\text { n.s. }\end{array}$ \\
\hline Motor leaning and coordination & & & & & & & & & & & & & & & & & & & & & \\
\hline Rotarod (latency to fall [s]) & & & & & & & & 13 & Genoty & $p=0.2, F$ & 1.383 & Genotyp & pe: $p=0.4, F$ & 0.834 & & & & & & & \\
\hline Motor strength & & & & & & & & & & & & & & & & & & & & & \\
\hline Grip strength (grip strength [P]) & & & & & & & & 16 & $95 \pm 6.3$ & $94 \pm 7.8$ & n.s. & $97 \pm 4.3$ & $97 \pm 3$ & n.s. & & & & & & & \\
\hline Hearing & & & & & & & & & & & & & & & & & & & & & \\
\hline $\begin{array}{l}\text { Hearing curve (startle amplitude } \\
[\mathrm{AU}])\end{array}$ & & & & & & & & 13 & $\begin{array}{l}\text { Genotyp } \\
\text { (TG: i }\end{array}$ & $\begin{array}{l}p=0.003, \\
\text { creased st }\end{array}$ & $\begin{array}{l}=15.53 \\
\text { rtle) }\end{array}$ & $\begin{array}{r}\text { Genoty } \\
\text { (TG: in }\end{array}$ & ype: $p=0.01$ & $\begin{array}{l}F=12 \\
\text { rtle) }\end{array}$ & & & & & & & \\
\hline Vision & & & & & & & & & & & & & & & & & & & & & \\
\hline $\begin{array}{l}\text { Visual cliff (preference for "ground" } \\
\text { [\%]) }\end{array}$ & & & & & & & & 15 & $52 \pm 0.7$ & $55 \pm 1.3$ & n.s. & $54 \pm 0.9$ & $52 \pm 1$ & n.s. & & & & & & & \\
\hline Olfaction & & & & & & & & & & & & & & & & & & & & & \\
\hline $\begin{array}{l}\text { Food finding test (latency to find } \\
\text { hidden cookie [s]) }\end{array}$ & & & & & & & & 18 & $83 \pm 29$ & $114 \pm 33$ & n.s. & $234 \pm 32$ & $270 \pm 15$ & n.s. & & & & & & & \\
\hline $\begin{array}{l}\text { Food finding test (latency to find } \\
\text { visible cookie [s]) }\end{array}$ & & & & & & & & 18 & $12 \pm 3.2$ & $11 \pm 2$ & n.s. & $14 \pm 2.2$ & $16 \pm 2.4$ & n.s. & & & & & & & \\
\hline Pain sensation & & & & & & & & & & & & & & & & & & & & & \\
\hline Hot plate (latency to lick [s]) & & & & & & & & 24 & & & & $18.6 \pm 1.3$ & $16.5 \pm 1.2$ & n.s. & & & & & & & \\
\hline Anhedonia-like behavior & & & & & & & & & & & & & & & & & & & & & \\
\hline $\begin{array}{l}\text { Sucrose preference test (preference } \\
\text { for sucrose [\%]) }\end{array}$ & & & & & & & & 20 & $69 \pm 3.9$ & $65 \pm 3.9$ & n.s. & $70 \pm 2.1$ & $64 \pm 2.5$ & n.s. & & & & & & & \\
\hline Sociability & & & & & & & & & & & & & & & & & & & & & \\
\hline $\begin{array}{l}\text { Social interaction: 3-partite chamber } \\
\text { (sociability index [AU]) }\end{array}$ & & & & & & & & 16 & $5.4 \pm 2.3$ & $5.8 \pm 4.3$ & n.s. & $7.9 \pm 3.4$ & $3.2 \pm 3.0$ & n.s. & 8 & $17 \pm 4.4$ & $13 \pm 5.2$ & n.s. & $22 \pm 3.1$ & $13 \pm 2.9$ & 0.05 \\
\hline Communication & & & & & & & & & & & & & & & & & & & & & \\
\hline Ultrasound vocalization (calls [\#]) & & & & & & & & & & & & & & & 13 & & & & $456 \pm 100$ & $434 \pm 103$ & n.s. \\
\hline Social competence & & & & & & & & & & & & & & & & & & & & & \\
\hline Nest building (Nest score [AU]) & & & & & & & & & & & & & & & & & & & & & \\
\hline Social dominance & & & & & & & & & & & & & & & & & & & & & \\
\hline Tube test (dominance score $[\mathrm{AU}]$ ) & & & & & & & & & & & & & & & & & & & & & \\
\hline
\end{tabular}




\begin{tabular}{|c|c|c|c|c|c|c|c|c|c|c|c|c|c|c|c|c|c|c|c|c|c|}
\hline \multirow{3}{*}{$\begin{array}{l}\text { Behavioral paradigms } \\
\text { (order according to domains) }\end{array}$} & \multicolumn{7}{|c|}{ Cohort 1} & \multicolumn{7}{|c|}{ Cohort 2} & \multicolumn{7}{|c|}{ Cohort 3} \\
\hline & \multirow[b]{2}{*}{$\mathrm{Age}^{\mathrm{a}}$} & \multicolumn{2}{|c|}{ Male } & \multicolumn{3}{|c|}{ Female } & \multirow[b]{2}{*}{$\mathbf{P}$} & \multirow[b]{2}{*}{$\mathrm{Age}^{\mathrm{a}}$} & \multicolumn{2}{|c|}{ Male } & \multicolumn{3}{|c|}{ Female } & \multirow[b]{2}{*}{$\mathbf{P}$} & \multirow[b]{2}{*}{$\mathrm{Age}^{\mathrm{a}}$} & \multicolumn{2}{|c|}{ Male } & \multicolumn{4}{|c|}{ Female } \\
\hline & & $\begin{array}{c}\text { WT } \\
(\mathrm{N}=14)\end{array}$ & $\begin{array}{c}\mathrm{TG} \\
(\mathrm{N}=18)\end{array}$ & $\mathbf{P}$ & $\begin{array}{c}\text { WT } \\
(\mathrm{N}=9)\end{array}$ & $\begin{array}{c}\mathrm{TG} \\
(\mathrm{N}=4)\end{array}$ & & & $\begin{array}{c}W T \\
(\mathrm{~N}=10-27)\end{array}$ & $\begin{array}{c}\text { TG } \\
(\mathrm{N}=6-22)\end{array}$ & $\mathbf{P}$ & $\begin{array}{c}W T \\
(N=15-27)\end{array}$ & $\begin{array}{c}\mathrm{TG} \\
(\mathrm{N}=14-37)\end{array}$ & & & $\begin{array}{c}W T \\
(\mathrm{~N}=10-28)\end{array}$ & $\begin{array}{c}\mathrm{TG} \\
(\mathrm{N}=10-12)\end{array}$ & $\mathbf{P}$ & $\begin{array}{c}W T \\
(\mathrm{~N}=10-14)\end{array}$ & $\begin{array}{c}\mathrm{TG} \\
(\mathrm{N}=10-21)\end{array}$ & $\mathbf{P}$ \\
\hline \multicolumn{22}{|l|}{ Aggression } \\
\hline $\begin{array}{l}\text { Resident-intruder paradigm } \\
\text { (latency to attack [s]); } 5 \mathrm{~min} \\
\text { session; FVB/N as intruder }\end{array}$ & \multirow[t]{3}{*}{8} & \multirow[t]{3}{*}{$200 \pm 33$} & \multirow[t]{3}{*}{$109 \pm 19$} & \multirow[t]{3}{*}{0.049} & & & & \multirow[t]{3}{*}{25} & \multirow[t]{3}{*}{$55 \pm 12$} & \multirow[t]{3}{*}{$49 \pm 8.1$} & \multirow[t]{3}{*}{0.7} & \multirow{3}{*}{\multicolumn{3}{|c|}{ no attack }} & 9 & $36 \pm 11$ & $19 \pm 3.6$ & 0.4 & & & \\
\hline $\begin{array}{l}\text { Resident-intruder paradigm } \\
\text { (latency to attack [s]]; } 5 \mathrm{~min} \\
\text { session; C57BL/6N as intruder }\end{array}$ & & & & & & & & & & & & & & & 16 & $209 \pm 22$ & $84 \pm 33$ & 0.002 & & & \\
\hline \multicolumn{11}{|l|}{$\begin{array}{l}\text { Resident-intruder paradigm } \\
\text { (latency to attack [s]); 10min } \\
\text { session with pre-warming; } \\
\text { C57BL/6N as intruder }\end{array}$} & & & & & & & & & & & \\
\hline Sensorimotor gating & & & & & & & & & & & & & & & & & & & & & \\
\hline Startle reactivity - PPI set up (startle & & & & & & & & & & & & & & & 7 & $8.5 \pm 0.8$ & $10.6 \pm 1.1$ & 0.06 & $6 \pm 0.6$ & $10 \pm 0.7$ & 0.0003 \\
\hline amplitude [AU]) & & & & & & & & & & & & & & & 16 & $9.6 \pm 1.2$ & $15 \pm 2$ & 0.05 & $5.4 \pm 1.3$ & $12 \pm 1.2$ & 0.005 \\
\hline $\begin{array}{l}\text { Prepulse inhibition (percentage } \\
\text { inhibition [\%]) }\end{array}$ & & & & & & & & 12 & Genoty & be: $p=0.3,1$ & $=1.314$ & Genoty & ype: $p=0.2$ & $=2.005$ & $\begin{array}{c}7 \\
16\end{array}$ & $\begin{array}{l}\text { Genoty } \\
\text { Genoty }\end{array}$ & $\begin{array}{l}\text { pe: } p=0.9, F \\
\text { pe: } p=0.5, F\end{array}$ & $=0.036$ & $\begin{array}{l}\text { Genotyp } \\
\text { Genotyp }\end{array}$ & $\begin{array}{l}\text { pe: } p=0.4, F \\
\text { pe: } p=0.6, F\end{array}$ & $=0.899$ \\
\hline Novel object recognition & & & & & & & & & & & & & & & & & & & & & \\
\hline $\begin{array}{l}\text { no delay paradigm (preference for } \\
\text { new object [\%]) } \\
30 \text { delay paradigm (preference for } \\
\text { new object [\%]) }\end{array}$ & & & & & & & & & & & & & & & & & & & & & \\
\hline Working memory & & & & & & & & & & & & & & & & & & & & & \\
\hline $\begin{array}{l}\text { Y-maze (percentage alternation [\%]) } \\
\text { Hole board (errors [\#]) }\end{array}$ & & & & & & & & & & & & & & & 7 & $55 \pm 3.0$ & $61 \pm 2.8$ & n.s. & $67 \pm 5.4$ & $61 \pm 3.4$ & n.s. \\
\hline Reference memory & & & & & & & & & & & & & & & & & & & & & \\
\hline Hole board (errors [\#]) & & & & & & & & & & & & & & & & & & & & & \\
\hline Home cage activity (LABORAS) & & & & & & & & & & & & & & & & & & & & & \\
\hline Locomotion frequency [\#] & & & & & & & & & & & & & & & 35 & $\begin{array}{l}6246 \\
\pm 724\end{array}$ & $\begin{array}{c}7822 \\
\pm 1155\end{array}$ & n.s. & $\begin{array}{l}20466 \\
\pm 2598\end{array}$ & $\begin{array}{l}6393 \\
\pm 919\end{array}$ & 0.0002 \\
\hline Immobility frequency [\#] & & & & & & & & & & & & & & & 35 & $\begin{array}{l}26679 \\
\pm 2510\end{array}$ & $\begin{array}{l}23322 \\
\pm 1678\end{array}$ & n.s. & $\begin{array}{c}5623 \\
\pm 2198\end{array}$ & $\begin{array}{l}23903 \\
\pm 3181\end{array}$ & 0.0002 \\
\hline Climbing frequency [\#] & & & & & & & & & & & & & & & 35 & $331 \pm 100$ & $196 \pm 45$ & n.s. & $451 \pm 78$ & $277 \pm 115$ & n.s. \\
\hline Seizure propensity & & & & & & & & & & & & & & & & & & & & & \\
\hline $\begin{array}{l}\text { PTZ- seizure induction (seizure score } \\
[A U])\end{array}$ & & & & & & & & 30 & $4.8 \pm 0.7$ & $9.1 \pm 2$ & 0.042 & $6 \pm 1$ & $8 \pm 1$ & 0.006 & & & & & & & \\
\hline Body weight [g] & 6 & $25 \pm 0.5$ & $25 \pm 0.3$ & n.s. & $21 \pm 0.3$ & $22 \pm 0.6$ & n.s. & 16 & $30 \pm 0.5$ & $30 \pm 0.7$ & n.s. & $25 \pm 0.5$ & $25 \pm 0.3$ & n.s. & 6 & $21 \pm 0.5$ & $21 \pm 0.7$ & n.s. & $17 \pm 0.5$ & $17 \pm 0.4$ & n.s. \\
\hline
\end{tabular}

Note: gray-shaded cells denote experiments that are presented in the figures. ${ }^{a}$ age in weeks. 
Table 1b: C57BL/6N mice (first part)

\begin{tabular}{|c|c|c|c|c|c|c|c|c|c|c|c|}
\hline \multirow{3}{*}{$\begin{array}{l}\text { Behavioral paradigms } \\
\text { (order according to domains) }\end{array}$} & \multicolumn{7}{|c|}{ Cohort 1 and 2} & \multirow{2}{*}{\multicolumn{4}{|c|}{$\begin{array}{l}\text { Cohort } 3 \\
\text { Male }\end{array}$}} \\
\hline & \multirow[b]{2}{*}{$\mathrm{Age}^{\mathrm{a}}$} & \multicolumn{2}{|c|}{ Male } & \multicolumn{3}{|c|}{ Female } & \multirow[b]{2}{*}{$\mathbf{P}$} & & & & \\
\hline & & $\begin{array}{c}W T \\
(\mathrm{~N}=10-26)\end{array}$ & $\begin{array}{c}\mathrm{TG} \\
(\mathrm{N}=12-13) \\
\end{array}$ & $\mathbf{P}$ & $\begin{array}{c}W T \\
(N=9-28)\end{array}$ & $\begin{array}{c}\mathrm{TG} \\
(\mathrm{N}=11-13)\end{array}$ & & $\mathrm{Age}^{\mathrm{a}}$ & $\underset{(N=16)}{W T}$ & $\underset{(N=10)}{T G}$ & $\mathbf{P}$ \\
\hline \multicolumn{12}{|l|}{ Anxiety-like behavior } \\
\hline $\begin{array}{l}\text { Elevated plus maze (time in open } \\
\text { versus closed arms [s]) }\end{array}$ & 5 & Genotype: & $: p=0.07, F=$ & $=3.398$ & Genotyp & $e: p=0.6, F=c$ & 0.244 & & & & \\
\hline \multicolumn{12}{|l|}{ Activity } \\
\hline Open field (total distance [m]) & 5 & $55.3 \pm 2$ & $58.1 \pm 3$ & n.s. & $60 \pm 1.9$ & $59 \pm 2.7$ & n.s. & & & & \\
\hline \multicolumn{12}{|l|}{ Exploratory behavior } \\
\hline Hole board (holes visited $[\#]$ ) & 7 & $11 \pm 1$ & $12 \pm 1.7$ & n.s. & $18 \pm 2.5$ & $20 \pm 2.9$ & n.s. & & & & \\
\hline \multicolumn{12}{|l|}{ Impulsivity } \\
\hline $\begin{array}{l}\text { Marble burying and digging (marbles } \\
\text { buried [\#]) }\end{array}$ & 8 & $9.5 \pm 1.4$ & $8.2 \pm 2.2$ & n.s. & $5.9 \pm 0.8$ & $7.7 \pm 1.2$ & n.s. & & & & \\
\hline \multicolumn{12}{|l|}{ Motor leaning and coordination } \\
\hline Rotarod (latency to fall [s]) & 6 & Genotype: & $: p=0.4, F=$ & 0.552 & Genotype & $e: p=0.8, F=c$ & 0.054 & & & & \\
\hline \multicolumn{12}{|l|}{ Motor strength } \\
\hline Grip strength (grip strength [P]) & 30 & $134 \pm 3.9$ & $138 \pm 5.8$ & n.s. & $123 \pm 3.2$ & $117 \pm 5.3$ & n.s. & & & & \\
\hline \multicolumn{12}{|l|}{ Hearing } \\
\hline Hearing curve (startle amplitude $[\mathrm{AU}]$ ) & 7 & Genotype: & $p=0.2, F=$ & 1.434 & Genotype & $e: p=0.1, F=$ & 2.773 & & & & \\
\hline \multicolumn{12}{|l|}{ Vision } \\
\hline $\begin{array}{l}\text { Visual cliff (preference for "ground" } \\
\text { [\%]) }\end{array}$ & 10 & $53 \pm 1.3$ & $53 \pm 1.3$ & n.s. & $52 \pm 1.3$ & $56 \pm 3.4$ & n.s. & & & & \\
\hline \multicolumn{12}{|l|}{ Olfaction } \\
\hline $\begin{array}{l}\text { Food finding test (latency to find } \\
\text { hidden cookie }[s] \text { ) }\end{array}$ & 16 & $107 \pm 15$ & $89 \pm 17$ & n.s. & $96 \pm 14$ & $113 \pm 30$ & n.s. & & & & \\
\hline $\begin{array}{l}\text { Food finding test (latency to find } \\
\text { visible cookie [s]) }\end{array}$ & 16 & $8.6 \pm 1.5$ & $12 \pm 3.5$ & n.s. & $6.5 \pm 1.8$ & $7.5 \pm 2.0$ & n.s. & & & & \\
\hline \multicolumn{12}{|l|}{ Pain sensation } \\
\hline Hot plate (latency to lick [s]) & 32 & $13 \pm 0.6$ & $15 \pm 1.2$ & n.s. & $16 \pm 0.7$ & $15 \pm 1.2$ & n.s. & & & & \\
\hline \multicolumn{12}{|l|}{ Anhedonia-like behavior } \\
\hline $\begin{array}{l}\text { Sucrose preference test (preference } \\
\text { for sucrose [\%]) }\end{array}$ & 14 & $91 \pm 1.3$ & $88 \pm 1.4$ & n.s. & $75 \pm 2.6$ & $71 \pm 4.9$ & n.s. & & & & \\
\hline \multicolumn{12}{|l|}{ Sociability } \\
\hline $\begin{array}{l}\text { Social interaction: 3-partite chamber } \\
\text { (sociability index }[\mathrm{AU}] \text { ) }\end{array}$ & 12 & $6.4 \pm 1.8$ & $6.2 \pm 3.2$ & n.s. & $9.0 \pm 1.3$ & $7.2 \pm 3.4$ & n.s. & & & & \\
\hline \multicolumn{12}{|l|}{ Communication } \\
\hline Ultrasound vocalization (calls $[\#]$ ) & 13 & $126 \pm 33$ & $26 \pm 12$ & 0.03 & $345 \pm 57$ & $150 \pm 62$ & 0.08 & & & & \\
\hline \multicolumn{12}{|l|}{ Social competence } \\
\hline Nest building (Nest score $[\mathrm{AU}]$ ) & & & & & & & & 7 & $3.8 \pm 0.2$ & $2.4 \pm 0.5$ & 0.02 \\
\hline \multicolumn{12}{|l|}{ Social dominance } \\
\hline Tube test (dominance score $[\mathrm{AU}]$ ) & & & & & & & & 14 & $1.1 \pm 0.3$ & $0.4 \pm 0.2$ & 0.1 \\
\hline
\end{tabular}




\begin{tabular}{|c|c|c|c|c|c|c|c|c|c|c|c|}
\hline \multirow{3}{*}{$\begin{array}{l}\text { Behavioral paradigms } \\
\text { (order according to domains) }\end{array}$} & \multicolumn{7}{|c|}{ Cohort 1 and 2} & \multirow{2}{*}{\multicolumn{4}{|c|}{$\begin{array}{l}\text { Cohort } 3 \\
\text { Male }\end{array}$}} \\
\hline & \multirow[b]{2}{*}{$\mathrm{Age}^{\mathrm{a}}$} & \multicolumn{2}{|c|}{ Male } & \multicolumn{3}{|c|}{ Female } & \multirow[b]{2}{*}{$\mathbf{P}$} & & & & \\
\hline & & $\begin{array}{l}\text { WT } \\
(N=14)\end{array}$ & $\begin{array}{c}\mathrm{TG} \\
(\mathrm{N}=18)\end{array}$ & $\mathbf{P}$ & $\begin{array}{l}\text { WT } \\
(\mathrm{N}=9)\end{array}$ & $\begin{array}{c}\mathrm{TG} \\
(\mathrm{N}=4)\end{array}$ & & \multirow[t]{2}{*}{$\mathrm{Age}^{\mathrm{a}}$} & $\begin{array}{c}\text { WT } \\
(\mathrm{N}=10-27)\end{array}$ & $\begin{array}{c}\mathrm{TG} \\
(\mathrm{N}=6-22)\end{array}$ & $\mathbf{P}$ \\
\hline \multicolumn{11}{|l|}{ Aggression } & \\
\hline \multicolumn{12}{|l|}{$\begin{array}{l}\text { Resident-intruder paradigm (latency } \\
\text { to attack [s]); } 5 \text { min session; FVB/N as } \\
\text { intruder }\end{array}$} \\
\hline $\begin{array}{l}\text { Resident-intruder paradigm (latency } \\
\text { to attack [s]); } 5 \mathrm{~min} \text { session; C57BL/6N } \\
\text { as intruder }\end{array}$ & \multirow[t]{2}{*}{32} & \multirow{2}{*}{\multicolumn{2}{|c|}{ no attack }} & & & & & 13 & \multicolumn{3}{|c|}{ no attack } \\
\hline $\begin{array}{l}\text { Resident-intruder paradigm (latency } \\
\text { to attack [s]); } 10 \mathrm{~min} \text { session with pre- } \\
\text { warming; } C 57 \mathrm{BL} / 6 \mathrm{~N} \text { as intruder }\end{array}$ & & & & & & & & 14 & $349 \pm 52$ & $460 \pm 53$ & 0.06 \\
\hline \multicolumn{12}{|l|}{ Sensorimotor gating } \\
\hline $\begin{array}{l}\text { Startle reactivity - PPI set up (startle } \\
\text { amplitude }[\mathrm{AU}] \text { ) }\end{array}$ & 7 & $7.7 \pm 0.5$ & $7.7 \pm 0.6$ & n.s. & $6.8 \pm 0.4$ & $8.9 \pm 0.6$ & $6 \quad 0.01$ & & & & \\
\hline $\begin{array}{l}\text { Prepulse inhibition (percentage } \\
\text { inhibition [\%]) }\end{array}$ & 7 & Genotyp & $e: p=0.7, F$ & $F=0.135$ & Genotyp & $p=0.7$ & $F=0.115$ & & & & \\
\hline \multicolumn{12}{|l|}{ Novel object recognition } \\
\hline $\begin{array}{l}\text { no delay paradigm (preference for } \\
\text { new object [\%]) }\end{array}$ & 8 & $56 \pm 3.9$ & $66 \pm 3.7$ & n.s. & $53 \pm 2.4$ & $56 \pm 3.5$ & n.s. & & & & \\
\hline $\begin{array}{l}30^{\prime} \text { delay paradigm (preference for } \\
\text { new object [\%]) }\end{array}$ & 8 & $57 \pm 2.9$ & $51 \pm 4.4$ & n.s. & $54 \pm 2.5$ & $62 \pm 3.7$ & n.s. & & & & \\
\hline \multicolumn{12}{|l|}{ Working memory } \\
\hline Y-maze (percentage alternation [\%]) & 10 & $64 \pm 2.0$ & $63 \pm 4.2$ & n.s. & $63 \pm 2.1$ & $66 \pm 2.6$ & n.s. & & & & \\
\hline Hole board (errors [\#]) & 20 & \multicolumn{3}{|c|}{$\begin{array}{c}\text { Genotype: } p=0.07, F=3.511 \\
\text { (TG: more errors) }\end{array}$} & \multicolumn{3}{|c|}{ Genotype: $\mathrm{p}=0.8, \mathrm{~F}=0.0228$} & & & & \\
\hline \multicolumn{12}{|l|}{ Reference memory } \\
\hline Hole board (errors [\#]) & 20 & \multicolumn{3}{|c|}{$\begin{array}{l}\text { Genotype: } p=0.09, F=3.174 \\
\text { (TG: more errors) }\end{array}$} & \multicolumn{3}{|c|}{ Genotype: $p=0.6, F=0.262$} & & & & \\
\hline \multicolumn{12}{|l|}{ Home cage activity (LABORAS) } \\
\hline Locomotion frequency [\#] & 37 & $\begin{array}{l}5110 \\
\pm 279\end{array}$ & $\begin{array}{c}258 \\
4 \pm 220\end{array}$ & $P<0.0001$ & $\begin{array}{l}5423 \\
\pm 376\end{array}$ & $\begin{array}{c}3579 \pm \\
442\end{array}$ & 0.009 & & & & \\
\hline Immobility frequency [\#] & 37 & $\begin{array}{l}13921 \\
\pm 1258\end{array}$ & $\begin{array}{l}27359 \\
\pm 1250\end{array}$ & $P<0.0001$ & $\begin{array}{l}12266 \\
\pm 1121\end{array}$ & $\begin{array}{l}22697 \\
\pm 1118\end{array}$ & $P<0.0001$ & & & & \\
\hline Climbing frequency [\#] & 37 & $105 \pm 10$ & $49 \pm 12$ & 0.004 & $349 \pm 73$ & $75 \pm 21$ & 0.0003 & & & & \\
\hline \multicolumn{12}{|l|}{ Seizure propensity } \\
\hline $\begin{array}{l}\text { PTZ- seizure induction (seizure score } \\
[A U])\end{array}$ & 40 & $6.7 \pm 1.1$ & $10 \pm 0.8$ & 0.03 & $7.7 \pm 0.8$ & $8.1 \pm 1$ & 0.02 & & & & \\
\hline Body weight [g] & 12 & $27 \pm 0.4$ & $27 \pm 0.4$ & n.s. & $23 \pm 0.3$ & $23 \pm 0.4$ & n.s. & 14 & $29 \pm 0.6$ & $30 \pm 1.3$ & n.s. \\
\hline
\end{tabular}

Note: gray-shaded cells denote experiments that are presented in the figures. ${ }^{a}$ age in weeks. 
Table 2: Phenotype comparison of GRAS patients by MECP2 SNP rs2734647 genotypes

\begin{tabular}{|c|c|c|c|c|}
\hline Males (GRAS sample) ${ }^{a}$ & C & & $\mathbf{T}$ & $\begin{array}{c}P \text { value } \\
\left(F / Z / \chi^{2} \text { value }\right)^{k}\end{array}$ \\
\hline Target variables $^{c}$ & $\mathrm{~N}=491-608$ & & $N=73-109$ & \\
\hline Poor impulse control, Mean \pm SD [range] & $1.72 \pm 1.12[1-6]$ & & $1.39 \pm 0.76[1-4]$ & $0.0001(F=14.65)$ \\
\hline Excitement, Mean $\pm S D$ [range] & $2.05 \pm 1.30[1-7]$ & & $1.82 \pm 1.13[1-6]$ & $0.034(F=4.52)$ \\
\hline \multicolumn{5}{|l|}{ Control variables } \\
\hline \multicolumn{5}{|l|}{ Sociodemographic variables } \\
\hline Age (at examination), $y$, Mean $\pm S D$ [range] & $37.40 \pm 12.09[17-78]$ & & $37.02 \pm 11.99[21-71]$ & $0.736(Z=-0.34)$ \\
\hline 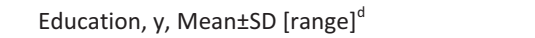 & $12.11 \pm 3.10[0-24]$ & & $11.32 \pm 2.48[8-18]$ & $0.020(Z=-2.33)$ \\
\hline Unemployment, No. (\%) & $107(17.8)$ & & $22(21.0)$ & $0.446\left(\chi^{2}=0.58\right)$ \\
\hline \multicolumn{5}{|l|}{ Clinical variables } \\
\hline PANSS general score, Mean $\pm S D$ [range ${ }^{e}$ & $30.57 \pm 10.82[15-81]$ & & $31.88 \pm 9.73[16-55]$ & $0.542(z=-0.61)$ \\
\hline PANSS negative score, Mean \pm SD [range] & $18.04 \pm 7.67[7-46]$ & & $19.23 \pm 7.31[7-40]$ & $0.070(Z=-1.81)$ \\
\hline PANSS positive score, Mean $\pm S D$ [range $]^{f}$ & $11.61 \pm 5.25[6-34]$ & & $11.39 \pm 5.15[6-30]$ & $0.667(Z=-0.43)$ \\
\hline Cognition composite score, Mean \pm SD $[\text { range }]^{\mathrm{g}}$ & $0.09 \pm 0.84[-2.4-2.2]$ & & $0.00 \pm 0.84[-2.1-1.7]$ & $0.543(F=0.37)$ \\
\hline Chlorpromazine equivalents, Mean \pm SD [range] & $688 \pm 644[0-4511]$ & & $725 \pm 734[0-6324]$ & $0.394(Z=-0.85)$ \\
\hline GAF score, Mean \pm SD [range] & $45.48 \pm 16.35[5-90]$ & & $46.94 \pm 15.45[15-85]$ & $0.375(Z=-0.89)$ \\
\hline Females (GRAS sample) $^{\mathrm{a}}$ & CC & CT & $\mathrm{TT}$ & $\begin{array}{c}P \text { value } \\
\left(F / Z / \chi^{2} \text { value }\right)^{\mathrm{b}}\end{array}$ \\
\hline Target variables $^{c}$ & $\mathrm{~N}=194-234$ & $N=73-91$ & $N=8-10$ & \\
\hline Poor impulse control, Mean \pm SD [range] & $1.73 \pm 1.15[1-7]$ & $1.57 \pm 0.94[1-5]$ & $1.60 \pm 1.07[1-4]$ & $0.729(F=0.31)$ \\
\hline Excitement, Mean \pm SD [range] & $2.11 \pm 1.30[1-7]$ & $1.93 \pm 1.31[1-7]$ & $1.50 \pm 0.53[1-2]$ & $0.277(F=1.29)$ \\
\hline \multicolumn{5}{|l|}{ Control variables } \\
\hline \multicolumn{5}{|l|}{ Sociodemographic variables } \\
\hline Age (at examination), $y$, Mean $\pm S D$ [range] & $43.41 \pm 12.90[18-76]$ & $41.82 \pm 12.47[19-72]$ & $43.52 \pm 12.10[20-58]$ & $0.657\left(\chi^{2}=0.84\right)$ \\
\hline${\text { Education, } y, \text { Mean } \pm S D[r a n g e]^{d}}^{d}$ & $12.61 \pm 3.36[8-27]$ & $12.14 \pm 2.83[8-21]$ & $12.15 \pm 3.76[8-19]$ & $0.750\left(\chi^{2}=0.58\right)$ \\
\hline Unemployment, No. (\%) & $25(10.9)$ & $12(13.6)$ & $4(40.0)$ & $0.176\left(\chi^{2}=1.83\right)$ \\
\hline \multicolumn{5}{|l|}{ Clinical variables } \\
\hline PANSS general score, Mean $\pm S D[\text { range }]^{e}$ & $33.04 \pm 12.10[15-69]$ & $32.38 \pm 12.25[15-72]$ & $31.30 \pm 10.64[19-54]$ & $0.872\left(\chi^{2}=0.28\right)$ \\
\hline PANSS negative score, Mean $\pm S D$ [range] & $18.13 \pm 8.50[7-44]$ & $17.82 \pm 8.11[7-36]$ & $19.30 \pm 9.36[7-39]$ & $0.904\left(\chi^{2}=0.20\right)$ \\
\hline PANSS positive score, Mean $\pm S D$ [range $]^{f}$ & $12.25 \pm 6.03[6-32]$ & $11.41 \pm 5.25[6-30]$ & $11.40 \pm 4.40[6-18]$ & $0.650\left(\chi^{2}=0.86\right)$ \\
\hline Cognition composite score, Mean \pm SD [range $]^{g}$ & $-0.02 \pm 0.83[-2.0-1.9]$ & $-0.19 \pm 0.90[-2.0-2.0]$ & $0.08 \pm 0.83[-1.5-1.1]$ & $0.090(F=2.43)$ \\
\hline Chlorpromazine equivalents, Mean \pm SD [range] & $677 \pm 839[0-7375]$ & $608 \pm 685[0-4375]$ & $640 \pm 512[150-1680]$ & $0.614\left(\chi^{2}=0.98\right)$ \\
\hline GAF score, Mean $\pm S D$ [range] & $45.44 \pm 19.07[12-90]$ & $47.07 \pm 18.60[8-90]$ & $48.80 \pm 14.73[25-70]$ & $0.472\left(\chi^{2}=1.50\right)$ \\
\hline
\end{tabular}

All p-values $\leq 0.05$ are set in boldface.

a Due to missing data, sample sizes vary.
${ }^{b}$ For statistical methods, Mann-Whitney U/Kruskal-Wallis test (women) or Chi ${ }^{2}$ tests and for models including covariates ANCOVAs (target variables and cognition composite score) were used.

CANCOVA with education, age at examination, negative symptoms (PANSS) and medication status (chlorpromazine equivalent) as covariates

Years spent in education system; patients currently in school or educational training included (score 0 ).

e Item 14 (target variable) excluded from sum score.

${ }^{\mathrm{f}}$ Item 4 (target variable) excluded from sum score.

${ }^{B}$ ANCOVA with age, negative symptoms (PANSS) and medication status (chlorpromazine equivalent) as covariates. 
Table 3: Phenotype comparison of GRAS patients by MECP2 SNP rs2239464 genotypes

\begin{tabular}{|c|c|c|c|c|}
\hline Males (GRAS sample) ${ }^{a}$ & G & & A & $\begin{array}{c}P \text { value } \\
\left(F / Z / \chi^{2} \text { value }\right)^{b}\end{array}$ \\
\hline Target variables $^{c}$ & $N=469-582$ & & $N=94-134$ & \\
\hline Poor impulse control, Mean $\pm S D$ [range] & $1.72 \pm 1.12[1-6]$ & & $1.44 \pm 0.81[1-4]$ & $0.001(F=10.84)$ \\
\hline Excitement, Mean \pm SD [range] & $2.05 \pm 1.31[1-7]$ & & $1.83 \pm 1.11[1-6]$ & $0.056(F=3.67)$ \\
\hline \multicolumn{5}{|l|}{ Control variables } \\
\hline \multicolumn{5}{|l|}{ Sociodemographic variables } \\
\hline Age (at examination), y, Mean $\pm S D$ [range] & $37.31 \pm 12.07[17-78]$ & & $37.27 \pm 12.12[21-71]$ & $0.918(Z=-0.10)$ \\
\hline Education, $y$, Mean $\pm S D$ [range $]^{d}$ & $12.10 \pm 3.10[0-24]$ & & $11.50 \pm 2.52[8-20]$ & $0.063(Z=-1.86)$ \\
\hline Unemployment, No. (\%) & $101(17.6)$ & & $28(21.5)$ & $0.294\left(\chi^{2}=1.10\right)$ \\
\hline \multicolumn{5}{|l|}{ Clinical variables } \\
\hline PANSS general score, Mean $\pm S D$ [range $]^{e}$ & $31.71 \pm 10.84[15-81]$ & & $31.36 \pm 9.83[16-55]$ & $0.966(Z=-0.04)$ \\
\hline PANSS negative score, Mean \pm SD [range] & $18.13 \pm 7.66[7-46]$ & & $18.61 \pm 7.48[7-40]$ & $0.420(Z=-0.81)$ \\
\hline PANSS positive score, Mean $\pm S D$ [range $]^{\dagger}$ & $11.67 \pm 5.29[6-34]$ & & $11.20 \pm 4.99[6-30]$ & $0.358(Z=-0.92)$ \\
\hline Cognition composite score, Mean $\pm S D$ [range ${ }^{\mathrm{g}}$ & $0.09 \pm 0.83[-2.4-2.2]$ & & $0.05 \pm 0.88[-2.1-1.9]$ & $0.842(F=0.40)$ \\
\hline Chlorpromazine equivalents, Mean $\pm S D$ [range] & $691 \pm 635[0-4511]$ & & $688 \pm 700[0-6324]$ & $0.936(\mathrm{Z}=-0.08)$ \\
\hline GAF score, Mean $\pm S D$ [range] & $45.11 \pm 16.26[5-90]$ & & $45.27 \pm 15.7[15-85]$ & $0.045(Z=-2.00)$ \\
\hline Females (GRAS sample) ${ }^{a}$ & GG & GA & AA & $\begin{array}{c}P \text { value } \\
\left(F / Z / \chi^{2} \text { value }\right)^{b}\end{array}$ \\
\hline Target variables $^{c}$ & $N=173-206$ & $N=94-117$ & $\mathrm{~N}=10-13$ & \\
\hline Poor impulse control, Mean $\pm S D$ [range] & $1.74 \pm 1.18[1-7]$ & $1.62 \pm 0.99[1-5]$ & $1.23 \pm 0.60[1-3]$ & $0.243(F=1.42)$ \\
\hline Excitement, Mean $\pm S D$ [range] & $2.12 \pm 1.29[1-7]$ & $1.98 \pm 1.31[1-7]$ & $1.62 \pm 1.12[1-5]$ & $0.416(F=0.88)$ \\
\hline \multicolumn{5}{|l|}{ Control variables } \\
\hline \multicolumn{5}{|l|}{ Sociodemographic variables } \\
\hline Age (at examination), $y$, Mean $\pm S D$ [range] & $43.65 \pm 13.11[18-76]$ & $42.01 \pm 12.15[19-72]$ & $42.66 \pm 12.37[20-58]$ & $0.665\left(\chi^{2}=0.82\right)$ \\
\hline Education, $y$, Mean $\pm S D$ [range $]^{d}$ & $12.66 \pm 3.32[8-27]$ & $12.22 \pm 3.05[8-21]$ & $11.96 \pm 3.28[8-19]$ & $0.538\left(x^{2}=1.24\right)$ \\
\hline Unemployment, No. (\%) & $20(10.0)$ & $17(14.5)$ & $4(30.8)$ & $0.056\left(\chi^{2}=5.77\right)$ \\
\hline \multicolumn{5}{|l|}{ Clinical variables } \\
\hline PANSS general score, Mean $\pm S D[\text { range }]^{e}$ & $32.92 \pm 12.42[15-69]$ & $33.11 \pm 11.79[15-72]$ & $30.23 \pm 11.42[19-54]$ & $0.690\left(\chi^{2}=0.74\right)$ \\
\hline PANSS negative score, Mean $₫ S D$ [range] & $18.07 \pm 8.49[7-44]$ & $18.20 \pm 8.37[7-39]$ & $17.62 \pm 8.48[7-39]$ & $0.961\left(\chi^{2}=0.08\right)$ \\
\hline PANSS positive score, Mean $\pm S D$ [range $]^{\dagger}$ & $12.28 \pm 5.99[6-32]$ & $11.74 \pm 5.55[6-32]$ & $10.00 \pm 4.26[6-18]$ & $0.440\left(\chi^{2}=1.64\right)$ \\
\hline Cognition composite score, Mean \pm SD $[\text { range }]^{\mathrm{g}}$ & $0.01 \pm 0.84[-2.0-1.9]$ & $-0.20 \pm 0.87[-2.0-2.0]$ & $0.03 \pm 0.75[-1.5-1.4]$ & $0.049(F=3.06)$ \\
\hline Chlorpromazine equivalents, Mean \pm SD [range] & $671 \pm 852[0-7375]$ & $638 \pm 702[0-4370]$ & $650 \pm 504[150-1680]$ & $0.811\left(\chi^{2}=0.41\right)$ \\
\hline GAF score, Mean $\pm S D$ [range] & $46.07 \pm 19.12[15-90]$ & $45.13 \pm 18.87[8-90]$ & $51.38 \pm 12.35[25-70]$ & $0.323\left(x^{2}=2.26\right)$ \\
\hline
\end{tabular}

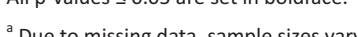

a Due to missing data, sample sizes vary.
${ }^{b}$ For statistical methods, Mann-Whitney U/Kruskal-Wallis test (women) or Chi ${ }^{2}$ tests and for models including covariates ANCOVAs (target variables and cognition composite score) were used.

'ANCOVA with education, age at examination, negative symptoms (PANSS) and medication status (chlorpromazine equivalent) as covariates.

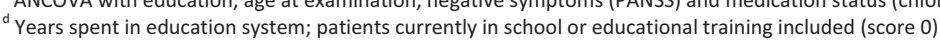

e Item 14 (target variable) excluded from sum score.

${ }^{8}$ ANCOVA with age, negative symptoms (PANSS) and medication status (chlorpromazine equivalent) as covariates. 
Table 4: Phenotype comparison of replication sample patients by MECP2 SNPs rs2734647 and rs2239464

\begin{tabular}{|c|c|c|c|}
\hline \multirow{2}{*}{ Males (replication sample) } & \multicolumn{2}{|c|}{ rs2734647 genotypes } & \multirow{2}{*}{$\begin{array}{c}P \text { value } \\
\left(Z / \chi^{2} \text { value }\right)^{2}\end{array}$} \\
\hline & C & $T$ & \\
\hline Target variables $^{b}$ & $\mathrm{~N}=322$ & $N=63$ & \\
\hline $\begin{array}{l}\text { Poor impulse control, Mean } \pm S D \text { [range] } \\
\text { Excitement, Mean } \pm S D \text { [range] }\end{array}$ & $\begin{array}{l}3.16 \pm 1.91[1-7] \\
3.79 \pm 1.60[1-7]\end{array}$ & $\begin{array}{l}2.86 \pm 1.93[1-6] \\
3.52 \pm 1.56[1-7]\end{array}$ & $\begin{array}{l}0.05(Z=-1.64) \\
0.09(Z=-1.33)\end{array}$ \\
\hline \multicolumn{4}{|l|}{ Control variables } \\
\hline $\begin{array}{l}\text { Age (at examination), y, Mean } \pm S D \text { [range] } \\
\text { Education rating, \% low, intermediate, high education level }\end{array}$ & $\begin{array}{l}35.34 \pm 10.91[19-65] \\
47.8 \%, 22.4 \%, 29.8 \%\end{array}$ & $\begin{array}{l}36.63 \pm 10.80[18-67] \\
42.9 \%, 30.2 \%, 27.0 \%\end{array}$ & $\begin{array}{l}0.16(Z=-1.01) \\
0.21\left(\chi^{2}=1.78\right)\end{array}$ \\
\hline \multirow[t]{2}{*}{ Males (replication sample) } & \multicolumn{2}{|c|}{ rs2239464 genotypes } & $P$ value \\
\hline & G & A & $\left(z / \chi^{2} \text { value }\right)^{a}$ \\
\hline Target variables $^{\mathrm{b}}$ & $N=308$ & $\mathrm{~N}=77$ & \\
\hline Poor impulse control, Mean \pm SD [range] & $3.19 \pm 1.92[1-7]$ & $2.82 \pm 1.89[1-6]$ & $0.03(\mathrm{Z}=-1.91)$ \\
\hline Excitement, Mean $\pm S D$ [range] & $3.83 \pm 1.60[1-7]$ & $3.42 \pm 1.58[1-7]$ & $0.02(z=-2.07)$ \\
\hline \multicolumn{4}{|l|}{ Control variables } \\
\hline $\begin{array}{l}\text { Age (at examination), y, Mean } \pm S D \text { [range] } \\
\text { Education rating \% low, intermediate, high education level }\end{array}$ & $35.28 \pm 11.01[19-65]$ & $36.66 \pm 10.40[18-67]$ & $0.12(z=-1.23)$ \\
\hline
\end{tabular}

All p-values $\leq 0.05$ are set in boldface.

${ }^{a}$ For statistical methods, Mann-Whitney $U$ or $C h i^{2}$ tests were used (all p-values one-sided).

Mann-Whitney $\mathrm{U}$ tests with standardized residuals from linear regression with target variables as dependent variables and age at examination and negative symptoms as independent variables. 
Tantra et al Figure 1
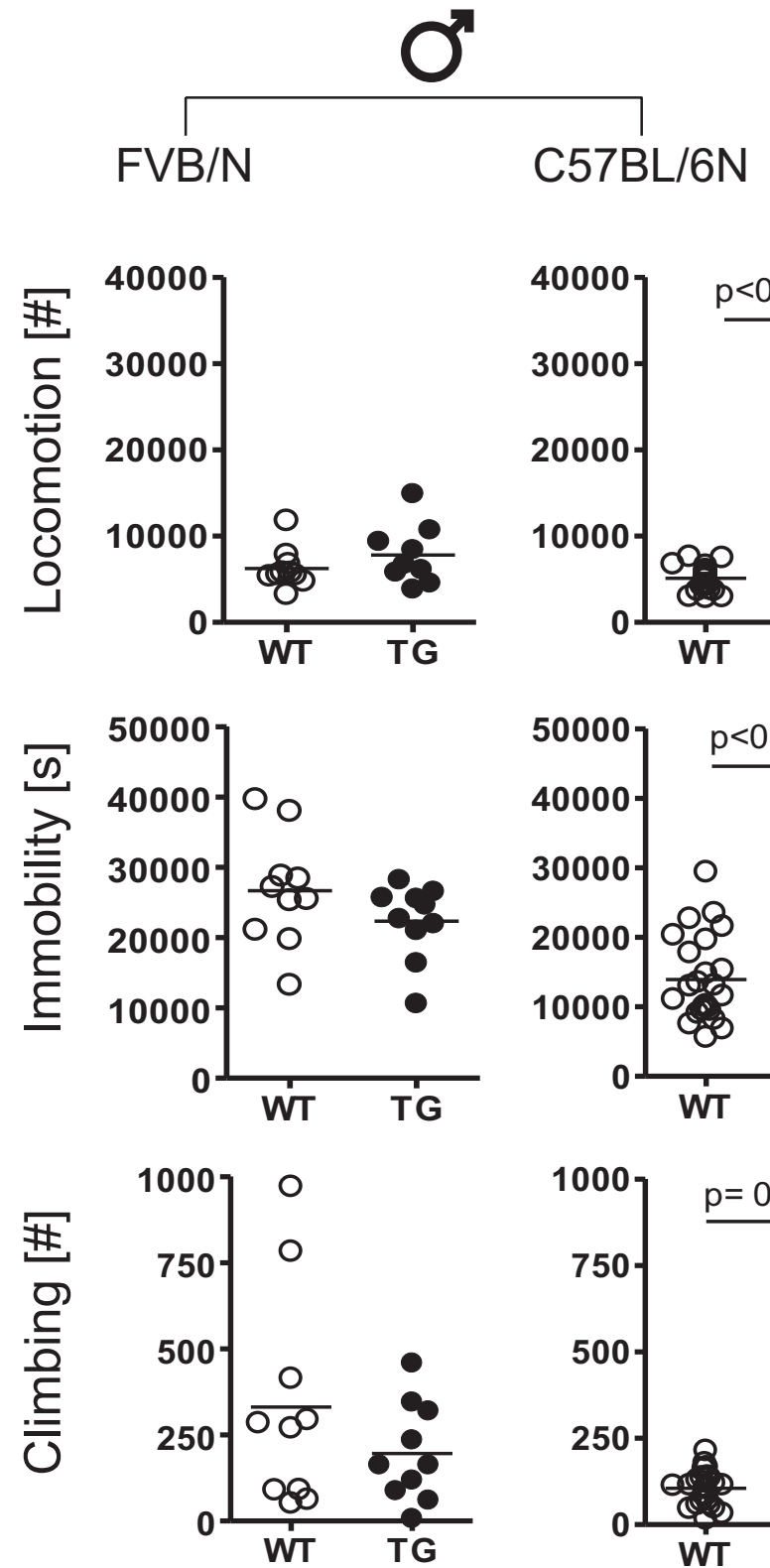
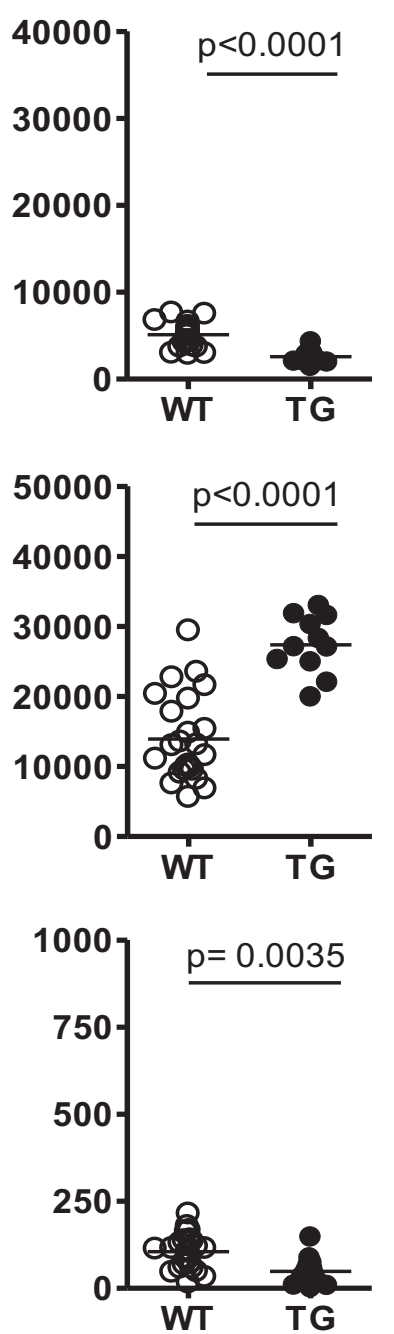
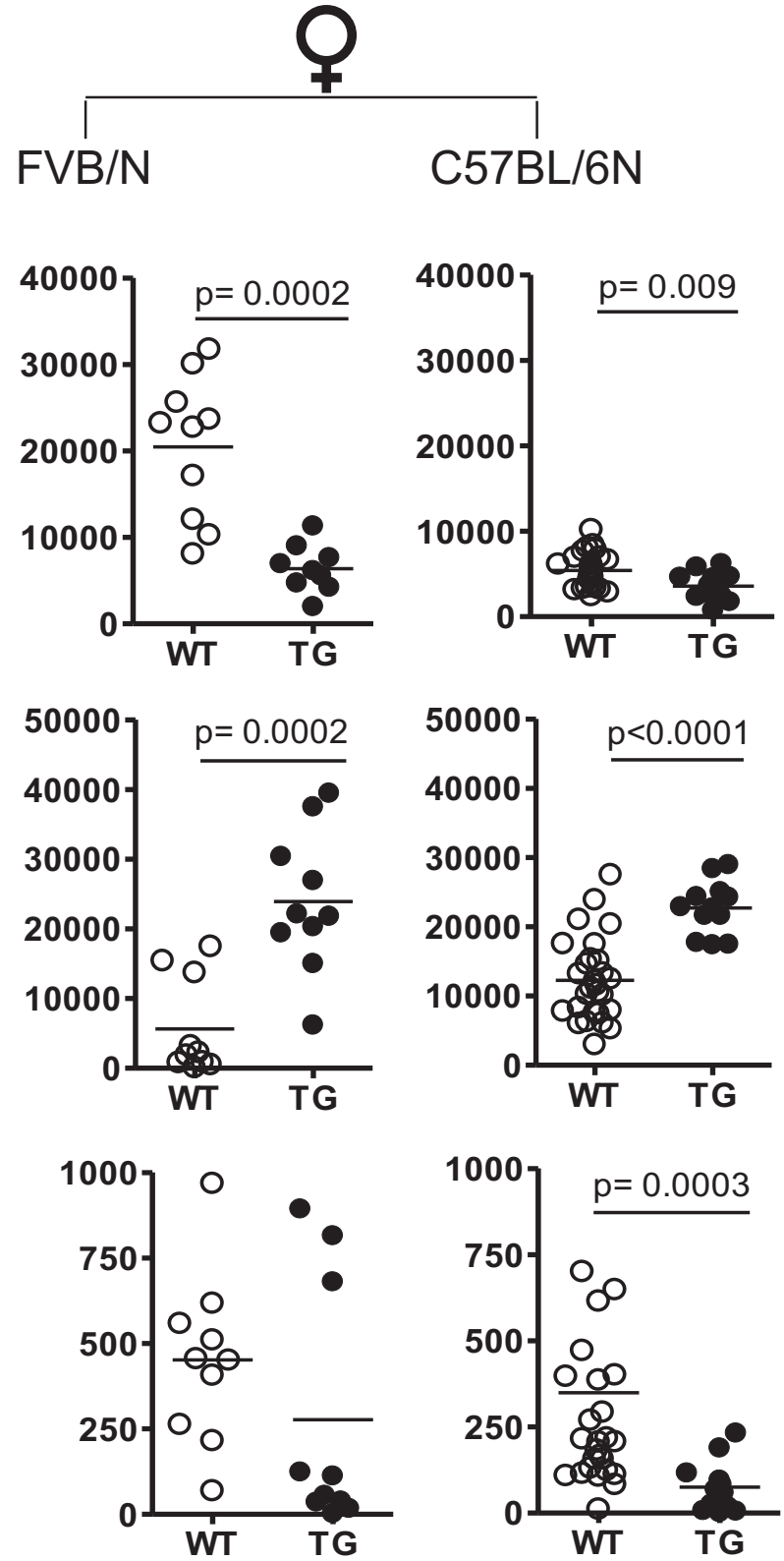
Tantra et al Figure 2

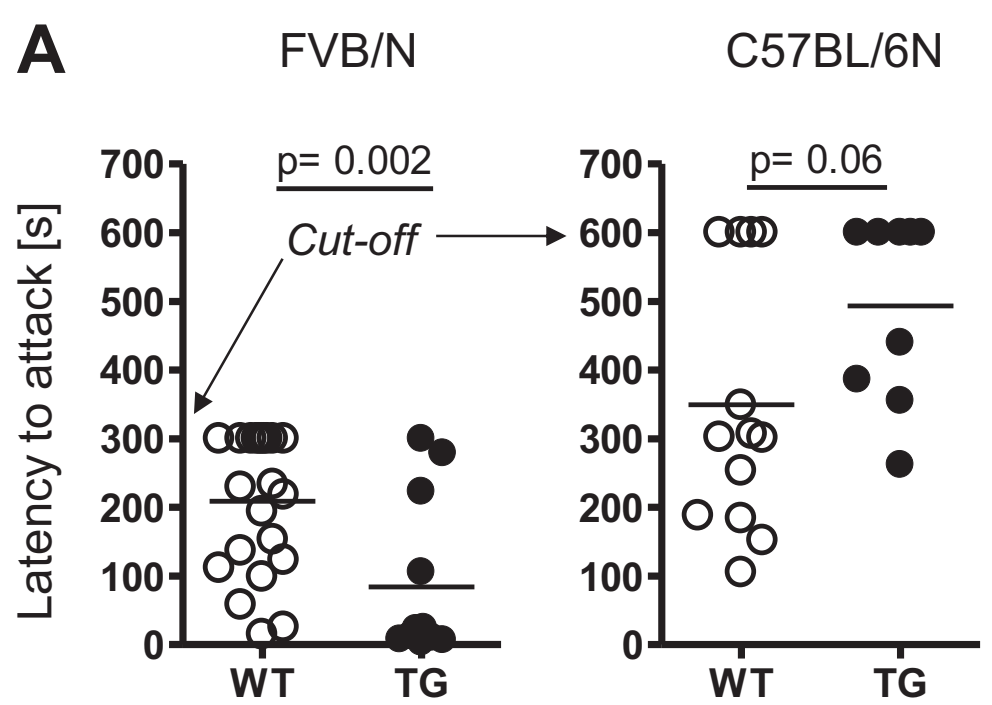

B

FVB/N

C57BL/6N
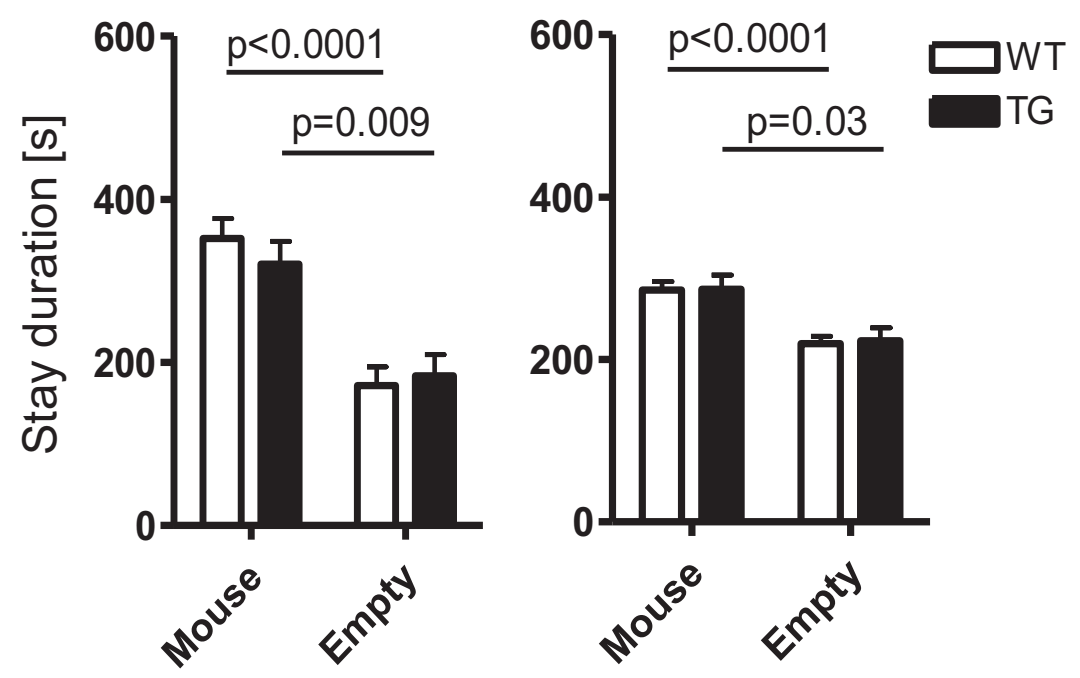
Tantra et al Figure 3

Follow/chase

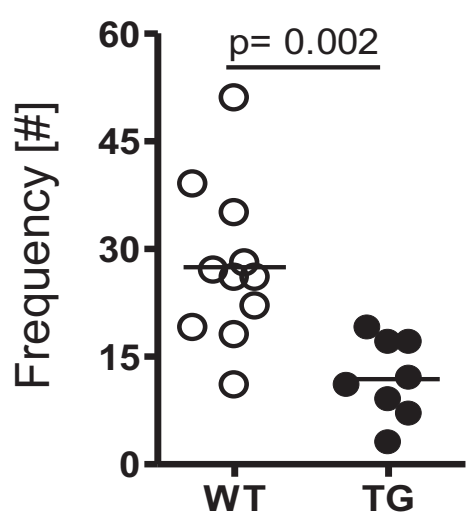

Follow/chase

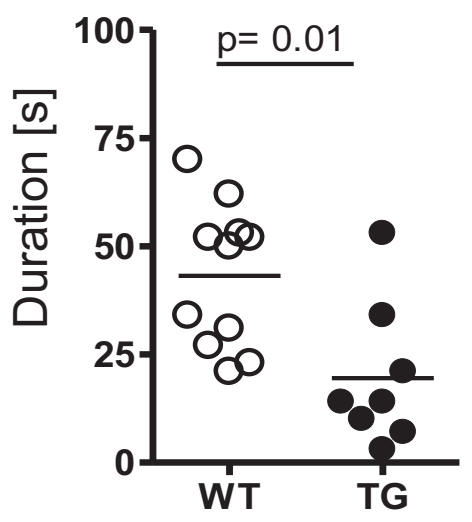

Nest building

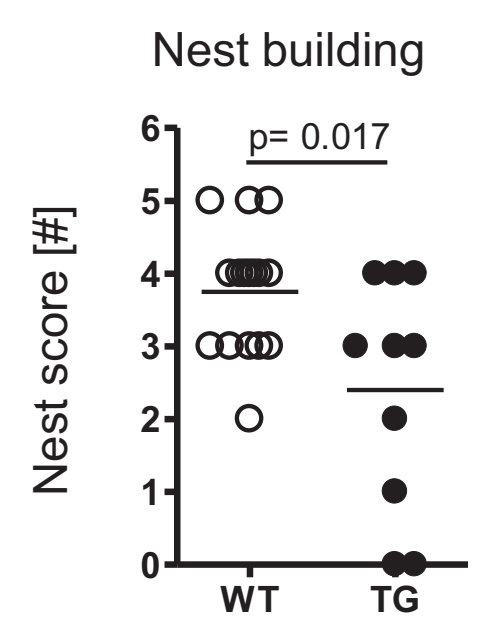

Nose/snout sniff

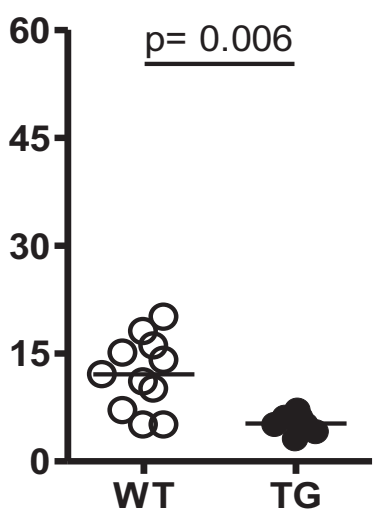

Nose/snout sniff

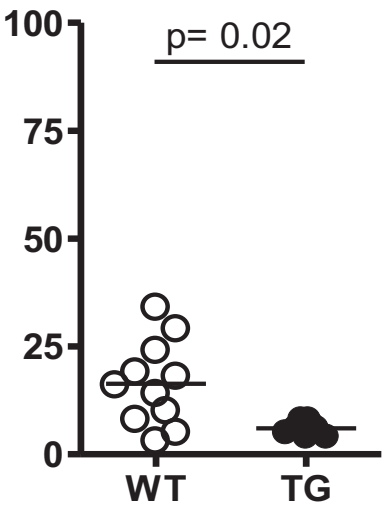

Social dominance

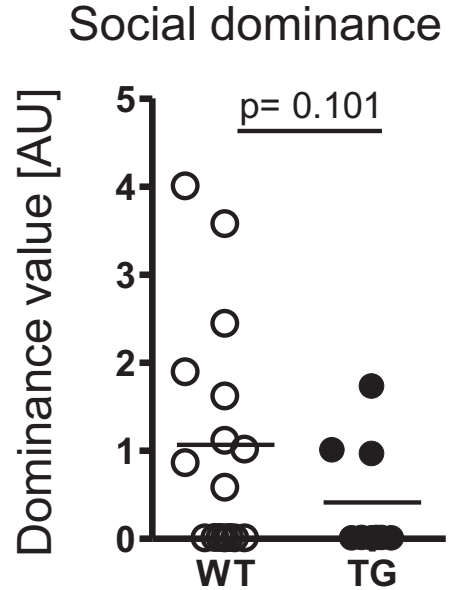

Anogenital sniff

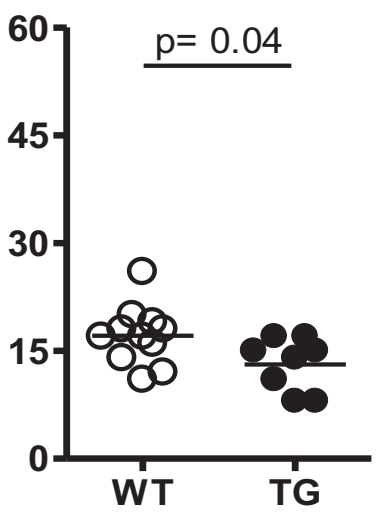

Anogenital sniff

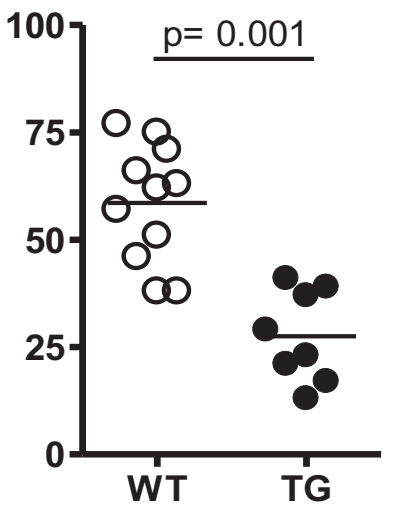

US vocalization

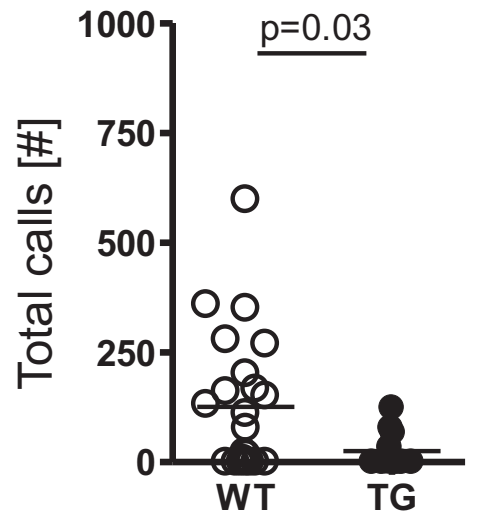


Tantra et al Figure 4
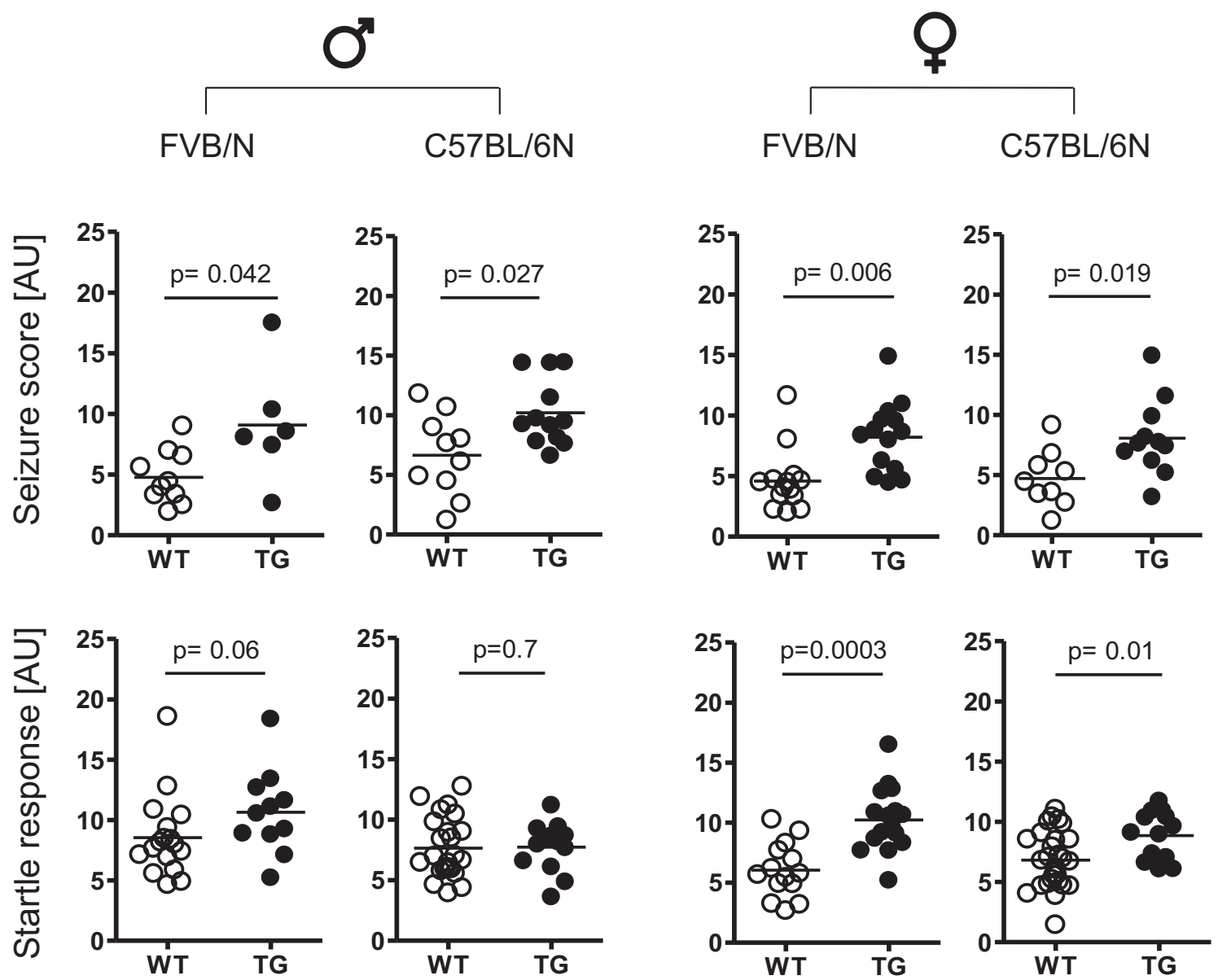
A

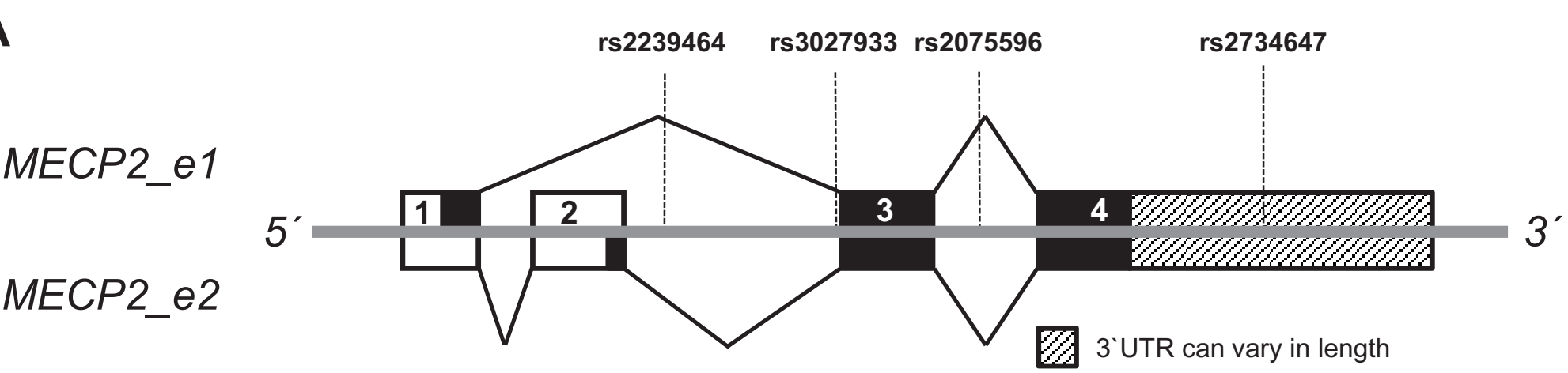

B

\begin{tabular}{l|rrr}
\hline \multirow{2}{*}{ SNP rs\# } & \multicolumn{3}{|c}{ Test for HWE deviation (females) } \\
\cline { 2 - 4 } & $\boldsymbol{P}$ (total) & $\boldsymbol{P}$ (controls) & \multicolumn{1}{c}{$\boldsymbol{P}$ (cases) } \\
\hline rs2239464 & 0.19 & 0.39 & 0.41 \\
rs3027933 & 0.51 & 0.49 & 1.00 \\
rs2075596 & 0.89 & 0.59 & 0.68 \\
rs2734647 & 1.00 & 0.74 & 0.70 \\
\hline
\end{tabular}

D

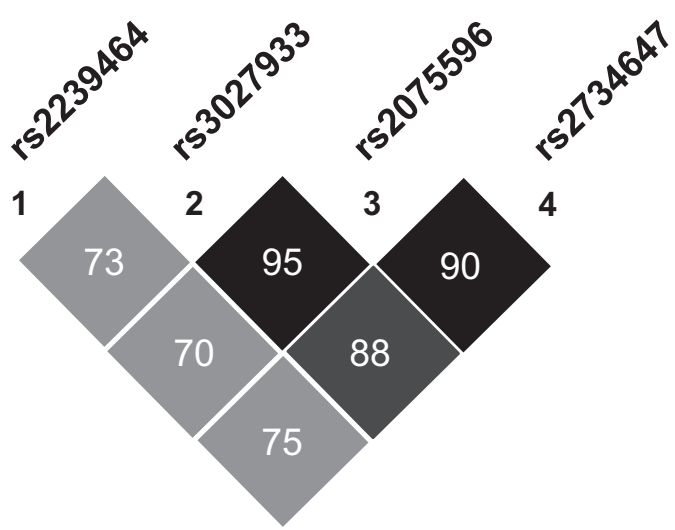

C

\begin{tabular}{|c|c|c|c|c|}
\hline \multirow{2}{*}{ SNP rs\# } & \multirow{2}{*}{ Genotype } & \multicolumn{2}{|c|}{$\mathbf{N}(\%)$} & \multirow{2}{*}{$\frac{P(\mathrm{OR},[95 \% \mathrm{Cl}])}{\text { allelic, d.f. }=1}$} \\
\hline & & GRAS patients & GRAS controls & \\
\hline \multicolumn{5}{|l|}{ Females } \\
\hline \multirow{3}{*}{ rs2239464 } & GG & $205(61.4)$ & $307(64.0)$ & \multirow{3}{*}{$0.45(1.10,[0.86-1.40])$} \\
\hline & GA & $117(35.0)$ & $158(32.9)$ & \\
\hline & AA & $12(3.6)$ & $15(3.1)$ & \\
\hline \multirow{3}{*}{ rs3027933 } & $\mathrm{CC}$ & $236(70.7)$ & $341(71.0)$ & \multirow{3}{*}{$0.80(1.04,[0.79-1.36])$} \\
\hline & CG & $90(26.9)$ & $130(27.1)$ & \\
\hline & GG & $8 \quad(2.4)$ & $9 \quad(1.9)$ & \\
\hline \multirow{3}{*}{ rs2075596 } & GG & $239(71.3)$ & $344(71.7)$ & \multirow{3}{*}{$0.75(1.05,[0.79-1.37])$} \\
\hline & GA & $87(26.0)$ & $127(26.5)$ & \\
\hline & AA & $9 \quad(2.7)$ & $9(1.9)$ & \\
\hline \multirow{3}{*}{ rs2734647 } & $\mathrm{CC}$ & $234(69.9)$ & $337(70.2)$ & \multirow{3}{*}{$0.77(1.04,[0.80-1.36])$} \\
\hline & CT & $91(27.2)$ & $132(27.5)$ & \\
\hline & TT & $10(3.0)$ & 11 (2.3) & \\
\hline \multicolumn{5}{|l|}{ Males } \\
\hline \multirow{2}{*}{ rs2239464 } & G & $582(81.4)$ & $617(80.3)$ & \multirow{2}{*}{$0.60(0.93,[0.72-1.21])$} \\
\hline & $\mathrm{A}$ & $133(18.6)$ & $151(19.7)$ & \\
\hline \multirow{2}{*}{ rs3027933 } & $C$ & $606(84.8)$ & $655(85.3)$ & \multirow{2}{*}{$0.77(1.04,[0.78-1.39])$} \\
\hline & G & $109(15.2)$ & $113(14.7)$ & \\
\hline \multirow{2}{*}{ rs2075596 } & G & $613(85.2)$ & $660(85.9)$ & \multirow{2}{*}{$0.81(1.04,[0.78-1.39])$} \\
\hline & A & $104(14.5)$ & $108(14.1)$ & \\
\hline \multirow{2}{*}{ rs2734647 } & C & $608(84.8)$ & $646(84.1)$ & \multirow{2}{*}{$0.71(0.95,[0.72-1.26])$} \\
\hline & $T$ & $109(15.2)$ & $122(15.9)$ & \\
\hline
\end{tabular}


Tantra et al Figure 6

A

\begin{tabular}{|c|c|c|c|c|c|}
\hline \multirow{2}{*}{ miRNA } & \multirow{2}{*}{\multicolumn{3}{|c|}{ Mature sequence }} & \multicolumn{2}{|c|}{$\Delta \Delta G$ value for rs2734647 } \\
\hline & & & & T-allele & C-allele \\
\hline hsa-miR-4711-3p & $45-$ & UCUUCUGGCUUGAU & -62 & -8.58 & -4.85 \\
\hline hsa-miR-511 & 16- & CUUUUGCUCUGCAGUCA & -36 & -7.82 & -4.37 \\
\hline hsa-miR-515-3p & $51-$ & GAGUGCCUUCUUUUGGAGCGUU & -72 & NA & -9.48 \\
\hline hsa-miR-519e & $52-$ & AAGUGCCUCCUUUUAGAGUGUU & -73 & -4.93 & -3.98 \\
\hline
\end{tabular}

B
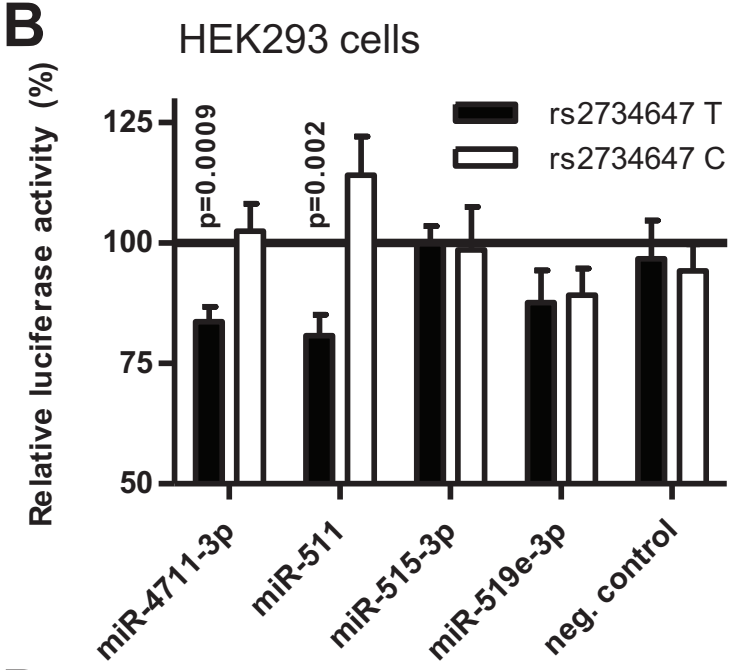

D

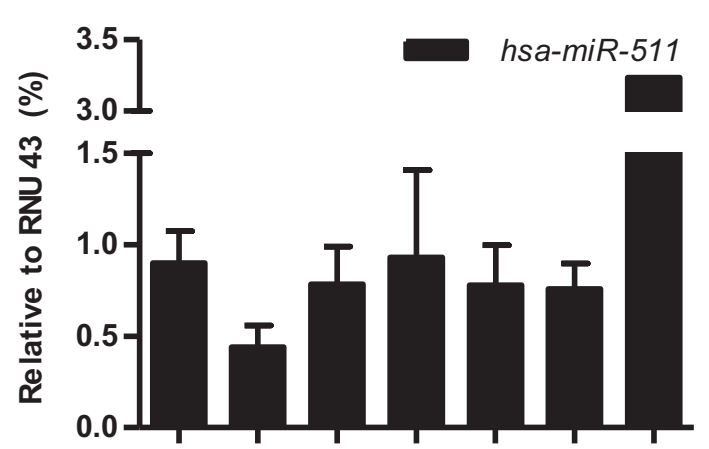

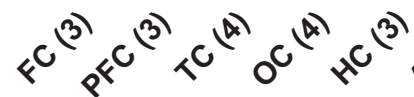

$\mathbf{F}$

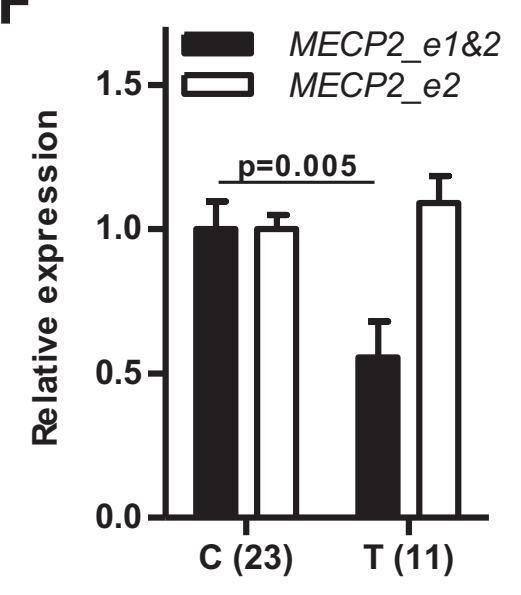

$\mathbf{G}$
C N2a cells

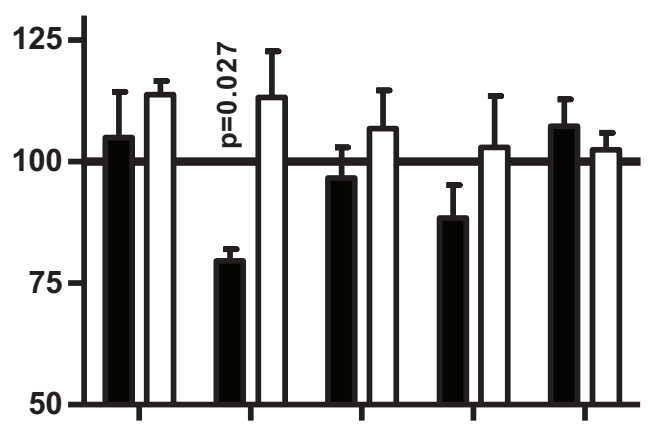

E
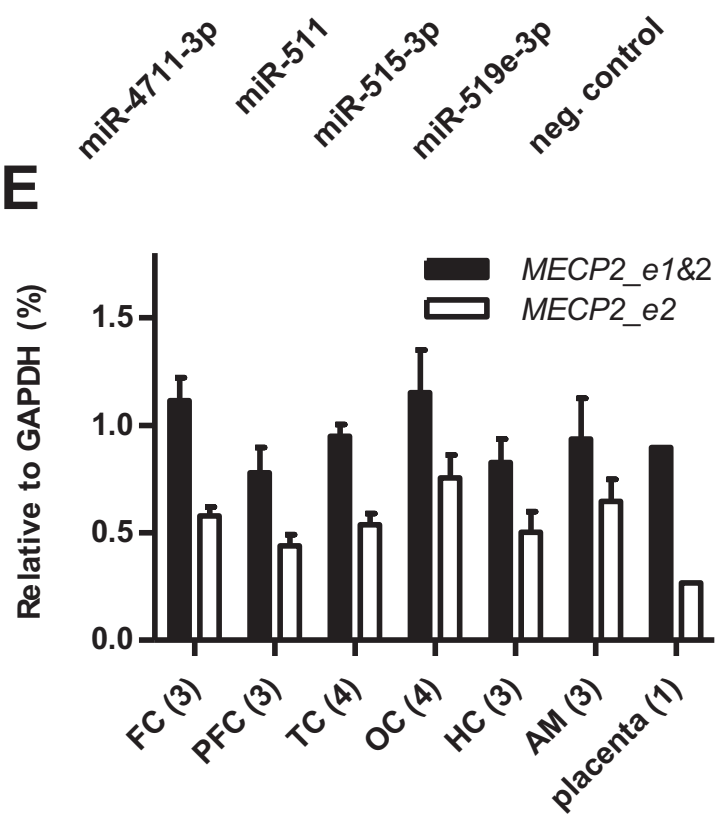

hsa-miR-511 3'- $\quad \ldots$. TTTTCTGT... $\quad$ - 5'

human MECP2 5'- UCCAUAUCAAAAGACACAGGGGAG- 3' mouse Mecp2 5'- CCCAUAUCAAAAGGCACAGGGGAC- 3' mmu-miR-511 3'- $\quad$...TTTTCCGT... 


\section{DISCUSSIONS AND SUMMARY}

Accumulating evidence consistently suggest neuropsychiatric conditions as results of complex interactions between multiple genetic factors and environmental factors. Studies of genetic mouse models are an appropriate approach to model such complexity, because genetic effects of behavior attributable to mutants are also subjects to the similar set of complex interaction between genetics and environmental factors (Bazzett 2008). Moreover, not only is the similarity of the mouse and human genomes remarkable (Waterston, Lindblad-Toh et al. 2002), behavioral domains addressed in this thesis are conserved between these two species. The present cumulative thesis addressed the relevance of behavioral characterization of genetic mouse models of complex human behavioral disorders such as psychiatric diseases, to identify the role of MECP2 and BAIAP3 and their common variants in modulating behavioral phenotypes.

In the first project, basic behavioral characterization revealed an increased anxiety, especially pronounced in female mice, due to the absence of Baiap3. On the pathological level, it was shown that Baiap3 deficiency increased the proneness to seizures induced by pentylenetetrazole, a non-competitive GABA antagonist. Studying the phenotype/ genotype relationships within the GRAS population, a significant association of BAIAP3 risk genotypes with anxiety in women, and surprisingly, benzodiazepine abuse in men was established. Upon characterizations of Baiap3 deficient mice for their response to benzodiazepines, it was established that the tolerance to diazepam developed more quickly in male, but not female Baiap3 deficient mice. Also, on the cellular level, an increase in basal network activity, together with a lack of homeostatic adaptation to diazepam were detected in hypothalamus slices of Baiap3 deficient mice.

In summary, it was shown in the first project that (1) the absence of Baiap3 in mice was attributable to increased anxiety and proneness to seizures, (2) Baiap3 deficiency in male, but not female mice led to a faster development of tolerance to diazepam, (3) a lack of homeostasis adaptation to diazepam in hypothalamus slices of Baiap3 deficient mice was a cellular consequence of Baiap3 deletion, (4) two common genetic variants of human BAIAP3 were associated with anxiety in women and benzodiazepine abuse in men of the GRAS population. Together, this 
translational approach yielded the identification of BAIAP3/Baiap3 as a genetic risk marker that is associated with anxiety and benzodiazepine abuse, in a genderspecific manner. For now, the practical implication of these results is limited, as these data are based on a phenotype-based genetic association study performed in a schizophrenic population. Hence, replications in non-schizophrenic populations are the requirement in order to apply these findings to the general population and would further substantiate the significance of these findings in elucidating mechanisms underlying anxiety and benzodiazepine use disorder. Nevertheless, these findings deliver the first evidence for BAIAP3 as a common risk factor in the emergence of anxiety and drug dependence.

In the second and third project, a detailed behavioral characterization of mice overexpressing a transcriptional regulator protein, Mecp2 at $\sim 1.5$ fold WT level led to the identification of elevated susceptibility to seizures and altered territorial aggression as the primary consequences of subtle increased of Mecp2 level. Evaluation of the quantitative effect of MECP2 expression level on phenotype is relevant, as both increased and reduced expression of MECP2 cause neurological disorders, termed MECP2 duplication and Rett syndrome (Amir, Van den Veyver et al. 1999; Moretti and Zoghbi 2006; Ramocki, Tavyev et al. 2010; Van Esch 2012).

The first project aimed to identify the consequences of mild alterations in the level of Mecp2, only at $\sim 1.5$ fold of the endogenous Mecp2. We argued that a mouse model with only mild overexpression of Mecp2 may aid to identify and characterize the earliest pathologies emerged due to gain-of-function properties of Mecp2, before the major phenotypes of disorders such as MECP2 duplication syndrome appears. The transgenic mouse model was established on the FVB/N genetic background. Evaluation of the neuronal parameters revealed (1) a reduction in the number of tertiary branching sites and increased spine density and (2) altered calcium homeostasis as cellular consequences of mild Mecp2 overexpression. Neonatal behavioral characterization of male transgenic mice did not reveal any abnormalities. Also, activity (locomotion), anxiety and exploratory behavior as well as motor and sensory function, including sensorimotor gating were unaffected by the mild increase of Mecp2 level. A higher tendency of male transgenic mice to bite was observed during daily handling. To quantify this observation, a resident-intruder paradigm for testing aggression was performed and revealed an increased aggression phenotype 
of male transgenic mice. Repeatedly, spontaneous seizures were observed in transgenic mice. When the mice were tested for their susceptibility to seizure induced by PTZ, increased PTZ-induced seizure activity was detected in transgenic male, but not in wild-type mice. In agreement with the seizure phenotype in transgenic mice, transgenic neurons treated with PTZ also demonstrated a marked increase in amplitude and frequency of calcium spikes, which are considered as a correlate of epileptic seizures.

Together, Mecp2 expressed at $\sim 1.5$ fold endogenous level led to (1) a reduction in the number of branching sites, (2) increased spine density, (3) alteration of the calcium homeostasis, (4) a higher susceptibility to PTZ-induced seizure and (5) a more aggressive phenotype in male transgenic mice of the FVB/N background. In conclusion, based on ex vivo and in vivo data, it was shown that epileptic seizures are manifested as the first symptom when Mecp2 is mildly overexpressed in mice.

In the frame of the third project, transgenic mice with $~ 1.5$ fold Mecp2 expression of the $\mathrm{FVB} / \mathrm{N}$ background were backcrossed for 10 generations to the C57BL/6N background to create a congenic strain. The third project was designed, among others, to explore the role of gender and genetic background (FVB/N versus C57BL/6N) for phenotypes associated with mild Mecp2 overexpression in mice, such as altered aggression, spontaneous home cage behavior and predisposition to seizure. The susceptibility to seizures remained as a pathological consequence of mildly overexpressing Mecp2 at 1.5fold endogenous level, observed across genders and genetic backgrounds. It was further shown that mild increased of Mecp2 expression altered aggression in male mice. While expressing the transgenic construct in mice of the $\mathrm{FVB} / \mathrm{N}$ background resulted in enhanced aggression, expressing the same transgenic construct in mice of the C57BL/6N background surprisingly led to reduced aggression, accompanied by decreased social interest. These results delineate the role of Mecp2 in modulating social behavior, however, the phenotypical outcomes appeared to be the subject of modifications by genetic backgrounds.

Adding to the picture of the phenotypical spectrums arose from Mecp2 dysfunctions (Chao and Zoghbi 2012), our results from the second and third projects suggest that $\sim 50 \%$ elevation of Mecp2 level resulted in relatively milder phenotypes, primarily characterized by an elevated predisposition to seizure and altered social behavior, 
while we did not observe any progressive neurological phenotype or premature lethality in the transgenic mice overexpressing Mecp2 at 1.5fold WT level (figure 3).

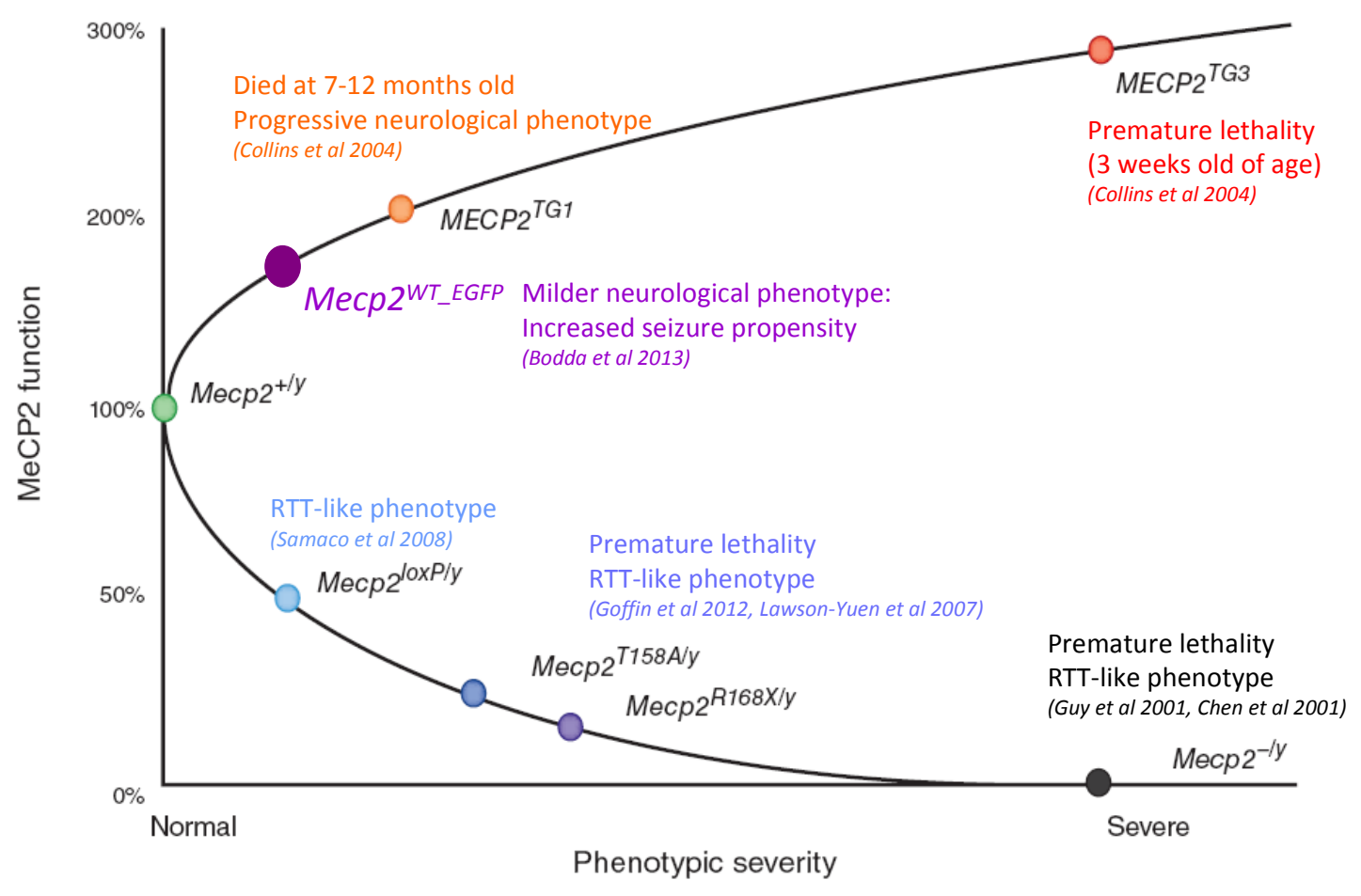

Adapted from Chao \& Zoghbi, 2012

Figure 3. Mild Mecp2 overexpression at $\sim 1.5$ fold endogenous level (Mecp2 ${ }^{\text {WT_EGFP }}$ ) led to a subtle neurological phenotype. In the spectrum of Mecp2 dysfunctions, it arrays between wild-type and transgenic mice with double Mecp2 level.

The discovery of Mecp2 influence on aggressive behavior in mice could also be identified in humans. We report a common genetic variant, SNPs in the 3'UTR of $M E C P 2$ which influence both the level of MECP2 expression and aggression in male, but not female individuals in the GRAS population. Putting results from mice and the phenotype-based genetic association studies together, we show that subtle changes of MECP2/Mecp2 expression level were sufficient to influence social behavior.

Overall, this thesis shows the practical implications of behavioral studies of transgenic mice with an elaborate behavioral repertoire that can be used to model phenotypes of complex human behavioral disorders. Behavioral characterizations of these mice gave aid to identify behavioral domains targeted by the genetic variations of the genes of interest, revealing the probable functional role of the genes within the frame of complex human behavioral disorders. The validity of the genetic mouse models 
described here was further increased by addressing the relevance of the findings in a schizophrenic (GRAS) population. Combining results from mouse studies and phenotype-based genetic association studies on GRAS database, it was shown that the functional consequences of genetic variations of the genes of interest were found to affect behavioral domains of both species: Baiap3/ BAIAP3 genetic variations modulate anxiety and the response to benzodiazepine, while Mecp2/ MECP2 variations influence social behavior. Last but not least, the significance of these findings could be further substantiated with more studies addressing the relevance of these findings in a more general (e.g. non-schizophrenic) population. 


\section{REFERENCES}

Abrahams, B. S. and D. H. Geschwind (2008). "Advances in autism genetics: on the threshold of a new neurobiology. "Nat Rev Genet 9(5): 341-355.

Adler, D. A., N. A. Quaderi, et al. (1995). "The X-Linked Methylated DNA-Binding Protein, Mecp2, Is Subject to X-Inactivation in the Mouse." Mammalian Genome 6(8): 491-492.

Al-Eitan, L. N., S. A. Jaradat, et al. (2012). "Custom genotyping for substance addiction susceptibility genes in Jordanians of Arab descent." BMC Res Notes 5: 497.

Amir, R. E., I. B. Van den Veyver, et al. (1999). "Rett syndrome is caused by mutations in X-linked MECP2, encoding methyl-CpG-binding protein 2." Nature Genetics 23(2): 185-188.

Argyropoulos, S. V. and D. J. Nutt (1999). "The use of benzodiazepines in anxiety and other disorders." European Neuropsychopharmacology 9: 407-412.

Baldwin, D. S., C. Allgulander, et al. (2012). "An international survey of reported prescribing practice in the treatment of patients with generalised anxiety disorder." World Journal of Biological Psychiatry 13(7): 510-516.

Bazzett, T. J. (2008). An introduction to behavior genetics. Sunderland, Mass., Sinauer Associates.

Bienvenu, T., A. Carrie, et al. (2000). "MECP2 mutations account for most cases of typical forms of Rett syndrome." Human Molecular Genetics 9(9): 1377-1384.

Bierut, L. J., P. A. Madden, et al. (2007). "Novel genes identified in a high-density genome wide association study for nicotine dependence." Hum Mol Genet 16(1): 24-35.

Bodda, C., M. Tantra, et al. (2013). "Mild Overexpression of Mecp2 in Mice Causes a Higher Susceptibility toward Seizures." Am J Pathol 183(1): 195-210.

Breman, A. M., M. B. Ramocki, et al. (2011). "MECP2 duplications in six patients with complex sex chromosome rearrangements." Eur J Hum Genet 19(4): 409-415.

Chahrour, M., S. Y. Jung, et al. (2008). "MeCP2, a key contributor to neurological disease, activates and represses transcription." Science 320(5880): 1224-1229.

Chahrour, M. and H. Y. Zoghbi (2007). "The story of Rett syndrome: from clinic to neurobiology." Neuron 56(3): 422-437.

Chao, H. T., H. Chen, et al. (2010). "Dysfunction in GABA signalling mediates autism-like stereotypies and Rett syndrome phenotypes." Nature 468(7321): 263-269.

Chao, H. T. and H. Y. Zoghbi (2012). "MeCP2: only 100\% will do." Nat Neurosci 15(2): 176-177.

Chen, R. Z., S. Akbarian, et al. (2001). "Deficiency of methyl-CpG binding protein-2 in CNS neurons results in a Rett-like phenotype in mice." Nature Genetics 27(3): 327-331.

Clocis, J. M. and V. Ferreira (2009). "Current use of benzodiazepines in anxiety disorders." Current Opinion in Psychiatry 22(1): 90-95.

Collins, A. L., J. M. Levenson, et al. (2004). "Mild overexpression of MeCP2 causes a progressive neurological disorder in mice." Hum Mol Genet 13(21): 2679-2689.

Compton Wm, T. Y. F. S. F. S. G. B. F. (2007). "Prevalence, correlates, disability, and comorbidity of dsm-iv drug abuse and dependence in the united states: Results from the national epidemiologic survey on alcohol and related conditions." Archives of General Psychiatry 64(5): 566-576. 
Crawley, J. N. (2007). What's wrong with my mouse? : behavioral phenotyping of transgenic and knockout mice. Hoboken, N.J., Wiley-Interscience.

Cristino, A. S., S. M. Williams, et al. (2013). "Neurodevelopmental and neuropsychiatric disorders represent an interconnected molecular system." Mol Psychiatry.

De Fillipis, B., L. Ricceri., et al. (2010). "Early postnatal behavioral changes in the Mecp2-308 truncation mouse model of Rett syndrome." Genes Brain Behav 9(2): 213-223.

del Gaudio, D., P. Fang, et al. (2006). "Increased MECP2 gene copy number as the result of genomic duplication in neurodevelopmentally delayed males." Genet Med 8(12): 784-792.

DeLorenzo, R. J., D. A. Sun, et al. (2007). "An in vitro model of stroke-induced epilepsy: elucidation of the roles of glutamate and calcium in the induction and maintenance of stroke-induced epileptogenesis." Int Rev Neurobiol 81: 59-84.

DeMartini, K. S. and K. B. Carey (2011). "The role of anxiety sensitivity and drinking motives in predicting alcohol use: A critical review." Clinical Psychology Review 31(1): 169-177.

Ducci, F. and D. Goldman (2012). "The genetic basis of addictive disorders." Psychiatr Clin North Am 35(2): 495-519.

El-Kordi, A., D. Winkler, et al. (2012). "Development of an autism severity score for mice using Nlgn4 null mutants as a construct-valid model of heritable monogenic autism." Behav Brain Res.

Fyffe, S. L., J. L. Neul, et al. (2008). "Deletion of Mecp2 in Sim1-expressing neurons reveals a critical role for MeCP2 in feeding behavior, aggression, and the response to stress." Neuron 59(6): 947-958.

Goffin, D., M. Allen, et al. (2012). "Rett syndrome mutation MeCP2 T158A disrupts DNA binding, protein stability and ERP responses." Nat Neurosci 15(2): 274-283.

Gratacos, M., I. Sahun, et al. (2007). "Candidate genes for panic disorder: insight from human and mouse genetic studies." Genes Brain Behav 6 Suppl 1: 2-23.

Guo, X., Z. Liu, et al. (2012). "Large scale association analysis for drug addiction: results from SNP to gene." ScientificWorldJournal 2012: 939584.

Guy, J., H. Cheval, et al. (2011). "The role of MeCP2 in the brain." Annu Rev Cell Dev Biol 27: 631-652.

Guy, J., J. Gan, et al. (2007). "Reversal of neurological defects in a mouse model of Rett syndrome." Science 315(5815): 1143-1147.

Guy, J., B. Hendrich, et al. (2001). "A mouse Mecp2-null mutation causes neurological symptoms that mimic Rett syndrome." Nat Genet 27(3): 322-326.

Hamilton, S. P. (2009). "Linkage and association studies of anxiety disorders." Depress Anxiety 26(11): 976-983.

Harari, O., J. C. Wang, et al. (2012). "Pathway analysis of smoking quantity in multiple GWAS identifies cholinergic and sensory pathways." PLoS One 7(12): e50913.

Hettema, J. M., C. A. Prescott, et al. (2005). "The structure of genetic and environmental risk factors for anxiety disorders in men and women." Arch Gen Psychiatry 62(2): 182-189.

Jamain, S., K. Radyushkin, et al. (2008). "Reduced social interaction and ultrasonic communication in a mouse model of monogenic heritable autism." Proc Natl Acad Sci U S A 105(5): 1710-1715.

Jian, L., L. Nagarajan, et al. (2006). "Predictors of seizure onset in Rett syndrome." Journal of Pediatrics 149(4): 542-547. 
Jones, P. L., G. J. C. Veenstra, et al. (1998). "Methylated DNA and MeCP2 recruit histone deacetylase to repress transcription." Nature Genetics 19(2): 187-191.

Kas, M. J. H., C. Fernandes, et al. (2006). "Genetics of behavioural domains across the neuropsychiatric spectrum; of mice and men." Mol Psychiatry 12(4): 324-330.

Kerr, B., M. Alvarez-Saavedra, et al. (2008). "Defective body-weight regulation, motor control and abnormal social interactions in Mecp2 hypomorphic mice." Hum Mol Genet 17(12): 1707-1717.

Kessler Rc, B. P. D. O. J. R. M. K. R. W. E. E. (2005). "Llfetime prevalence and age-of-onset distributions of dsm-iv disorders in the national comorbidity survey replication." $\underline{\text { Archives of }}$ General Psychiatry 62(6): 593-602.

Koch, H., K. Hofmann, et al. (2000). "Definition of Munc13-homology-domains and characterization of a novel ubiquitously expressed Munc13 isoform." Biochem J 349(Pt 1): 247-253.

Lawson-Yuen, A., D. Liu, et al (2007). "Ube3a mRNA and protein expression are not decreased in Mecp2R168X mutant mice." Brain Res 1180: 1-6.

Lee, S. S., M. Wan, et al. (2001). "Spectrum of MECP2 mutations in Rett syndrome." Brain Dev 23 Suppl 1: S138-143.

Lewis, J. D., R. R. Meehan, et al. (1992). "Purification, Sequence, and Cellular-Localization of a Novel Chromosomal Protein That Binds to Methylated DNA." Cell 69(6): 905-914.

Loat, C. S., S. Curran, et al. (2008). "Methyl-CpG-binding protein 2 polymorphisms and vulnerability to autism." Genes Brain Behav 7(7): 754-760.

Marmorstein, N. R. (2012). "Anxiety disorders and substance use disorders: different associations by anxiety disorder." J Anxiety Disord 26(1): 88-94.

McGraw, C. M., R. C. Samaco, et al. (2011). "Adult Neural Function Requires MeCP2." Science 333(6039): 186.

Mellén, M., P. Ayata, et al. (2012). "MeCP2 Binds to 5hmC Enriched within Active Genes and Accessible Chromatin in the Nervous System." Cell 151(7): 1417-1430.

Mineur, Y. S. and W. E. Crusio (2002). "Behavioral and neuroanatomical characterization of FVB/N inbred mice." Brain Res Bull 57(1): 41-47.

Minh, V. C. N., F. Du, et al. (2012). "MeCP2 Is Critical for Maintaining Mature Neuronal Networks and Global Brain Anatomy during Late Stages of Postnatal Brain Development and in the Mature Adult Brain." Journal of Neuroscience 32(29): 10021-10034.

Moretti, P., J. A. Bouwknecht, et al. (2005). "Abnormalities of social interactions and home-cage behavior in a mouse model of Rett syndrome." Hum Mol Genet 14(2): 205-220.

Moretti, P. and H. Y. Zoghbi (2006). "MeCP2 dysfunction in Rett syndrome and related disorders." Current Opinion in Genetics \& Development 16(3): 276-281.

Mount, R. H., T. Charman, et al. (2003). "Features of autism in Rett syndrome and severe mental retardation." Journal of Autism and Developmental Disorders 33(4): 435-442.

Moy, S. S., J. J. Nadler, et al. (2009). "Social approach in genetically engineered mouse lines relevant to autism." Genes Brain Behav 8(2): 129-142.

Mueller, T. I., M. E. Pagano, et al. (2005). "Long-term use of benzodiazepines in participants with comorbid anxiety and alcohol use disorders." Alcoholism-Clinical and Experimental Research 29(8): 1411-1418.

Nan, X. S., H. H. Ng, et al. (1998). "Transcriptional repression by the methyl-CpG-binding protein MeCP2 involves a histone deacetylase complex." Nature 393(6683): 386-389. 
O'Leary, T. P., R. K. Gunn, et al. (2013). "What are We Measuring When We Test Strain Differences in Anxiety in Mice?" Behavior Genetics 43(1): 34-50.

Pasche, S. (2012). "Exploring the Comorbidity of Anxiety and Substance Use Disorders." Current Psychiatry Reports 14(3): 176-181.

Pearson, B. L., E. B. Defensor, et al. (2012). "Mecp2 Truncation in Male Mice Promotes Affiliative Social Behavior." Behavior Genetics 42(2): 299-312.

Pelka, G.J., C. M. Watson, et al. (2006). "Mecp2 deficiency is associated with learning and cognitive deficits and altered gene activity in the hippocampal region of mice." Brain 129: 887-898.

Pietropaolo, S., A. Guilleminot, et al. (2011). "Genetic-background modulation of core and variable autistic-like symptoms in Fmr1 knock-out mice." PLoS One 6(2): e17073.

Pugh, P. L., S. F. Ahmed, et al. (2004). "A behavioural characterisation of the FVB/N mouse strain." Behavioural Brain Research 155(2): 283-289.

Radyushkin, K., K. Hammerschmidt, et al. (2009). "Neuroligin-3-deficient mice: model of a monogenic heritable form of autism with an olfactory deficit." Genes Brain Behav 8(4): 416-425.

Ramocki, M. B., S. U. Peters, et al. (2009). "Autism and other neuropsychiatric symptoms are prevalent in individuals with MeCP2 duplication syndrome." Ann Neurol 66(6): 771-782.

Ramocki, M. B., Y. J. Tavyev, et al. (2010). "The MECP2 duplication syndrome." $\underline{\text { Am J Med Genet A }}$ 152A(5): 1079-1088.

Ramocki, M. B. and H. Y. Zoghbi (2008). "Failure of neuronal homeostasis results in common neuropsychiatric phenotypes." Nature 455(7215): 912-918.

Ribbe, K., H. Friedrichs, et al. (2010). "The cross-sectional GRAS sample: A comprehensive phenotypical data collection of schizophrenic patients. "BMC Psychiatry 10(1): 91.

Samaco, R. C., J. D. Fryer, et al. (2008). "A partial loss of function allele of methyl-CpG-binding protein 2 predicts a human neurodevelopmental syndrome." Hum Mol Genet 17(12): 1718-1727.

Samaco, R. C., C. Mandel-Brehm, et al. (2009). "Loss of MeCP2 in aminergic neurons causes cellautonomous defects in neurotransmitter synthesis and specific behavioral abnormalities." Proc Natl Acad Sci U S A 106(51): 21966-21971.

Samaco, R. C., C. Mandel-Brehm, et al. (2012). "Crh and Oprm1 mediate anxiety-related behavior and social approach in a mouse model of MECP2 duplication syndrome." Nat Genet 44(2): 206211.

Samaco, R. C., C. M. McGraw, et al. (2013). "Female Mecp2(+/-) mice display robust behavioral deficits on two different genetic backgrounds providing a framework for pre-clinical studies." Hum Mol Genet 22(1): 96-109.

Schuckit, M. A. (2006). "Comorbidity between substance use disorders and psychiatric conditions." Addiction 101: 76-88.

Shahbazian, M., J. Young, et al. (2002). "Mice with truncated MeCP2 recapitulate many Rett syndrome features and display hyperacetylation of histone H3." Neuron 35(2): 243-254.

Shiratsuchi, T., K. Oda, et al. (1998). "Cloning and characterization of BAP3 (BAl-associated protein 3), a C2 domain-containing protein that interacts with BAl1." Biochemical and Biophysical Research Communications 251(1): 158-165.

Smith, J. P. and S. W. Book (2008). "Anxiety and substance use disorders: A review." The Psychiatric times 25(10): 19. 
Tecott, L. H. (2003). "The genes and brains of mice and men." American Journal of Psychiatry 160(4): 646-656.

Van Esch, H. (2012). "MECP2 Duplication Syndrome." Mol Syndromol 2(3-5): 128-136.

Wahlsten, D. (2011). Mouse behavioral testing : how to use mice in behavioral neuroscience. London ; Burlington, VT, Academic.

Waterston, R. H., K. Lindblad-Toh, et al. (2002). "Initial sequencing and comparative analysis of the mouse genome." Nature 420(6915): 520-562.

Wojcik, S. M. and N. Brose (2007). "Regulation of membrane fusion in synaptic excitation-secretion coupling: speed and accuracy matter." Neuron 55(1): 11-24.

Wojcik, S. M., M. Tantra, et al. (2013). "Genetic Markers of a Munc13 Protein Family Member, BAIAP3, Are Gender-Specifically Associated with Anxiety and Benzodiazepine Abuse in Mouse and Man." Mol Med.

Wolfer, D. P. and H. P. Lipp (2000). "Dissecting the behaviour of transgenic mice: is it the mutation, the genetic background, or the environment?" Experimental Physiology 85(6): 627-634.

Yasui, D. H., S. Peddada, et al. (2007). "Integrated epigenomic analyses of neuronal MeCP2 reveal a role for long-range interaction with active genes." Proc Natl Acad Sci U S A 104(49): 1941619421. 


\section{LIST OF PUBLICATIONS}

Wojcik $\mathrm{SM}^{\star}$, Tantra $\mathbf{M}^{\star}$, Stepniak B*, Man KM, Müller-Ribbe K, Begemann M, Ju A, Papiol S, Ronnenberg A, Gurvich A, Shin Y, Augustin I, Brose N, Ehrenreich H. Genetic Markers of a Munc13 Protein Family Member, BAIAP3, Are GenderSpecifically Associated with Anxiety and Benzodiazepine Abuse in Mouse and Man. Mol Med. 2013 May; 19:135-148.

Bodda $\mathrm{C}^{*}$, Tantra $\mathbf{M}^{*}$, Mollajew $\mathrm{R}$, Arunachalam JP, Laccone $\mathrm{FA}$, Can $\mathrm{K}$, Rosenberger A, Mironov SL, Ehrenreich $\mathrm{H}$ and Mannan AU. Mild overexpression of Mecp2 in mice causes a higher susceptibility toward seizures. Am J Pathol. 2013 Jul; 183(1):195-210.

Tantra $\mathbf{M}^{*}$, Hammer $C^{*}$, Kästner $A^{*}$, Dahm L, Begemann $M$, Bodda $C$, Hammerschmidt K, Giegling I, Stepniak B, Venzor AC, Konte B, Erbaba B, Hartmann A, Tarami A, Schulz-Schaeffer W, Rujescu D, Mannan AU, Ehrenreich H: Mild expression differences of MECP2 influencing aggressive social behavior. (Submitted)

${ }^{*}$ Equal contribution

Hammer C, Stepniak B, Schneider A, Papiol S, Tantra M, Begemann M, Siren A-L, Pardo LA, Sperling S, Joffry SM, Gurvich A, Jensen N, Ostmeier K, Lühder F, Probst C, Martens H, Gillis M, Saher G, Assogna F, Spalletta G, Stöcker W, Schulz TF, Nave $K-A$, Ehrenreich $H$. Neuropsychiatric disease relevance of circulating anti-NMDA receptor autoantibodies depends on blood brain barrier integrity. Mol Psychiatry (in press). 
9. APPENDIX 


\title{
ORIGINAL ARTICLE
}

\section{Neuropsychiatric disease relevance of circulating anti-NMDA receptor autoantibodies depends on blood-brain barrier integrity}

\author{
C Hammer ${ }^{1}$, B Stepniak ${ }^{1}$, A Schneider ${ }^{2,3,4}$, S Papiol ${ }^{1,3}$, M Tantra ${ }^{1,3}$, M Begemann ${ }^{1}$, A-L Sirén ${ }^{5}$, LA Pardo ${ }^{6}$, S Sperling $^{1}$, S Mohd Jofrry ${ }^{1}$,

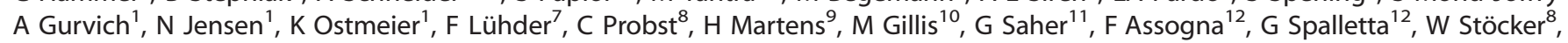 \\ TF Schulz ${ }^{10}$, K-A Nave ${ }^{3,11}$ and $\mathrm{H}$ Ehrenreich ${ }^{1,3}$
}

In 2007, a multifaceted syndrome, associated with anti-NMDA receptor autoantibodies (NMDAR-AB) of immunoglobulin-G isotype, has been described, which variably consists of psychosis, epilepsy, cognitive decline and extrapyramidal symptoms. Prevalence and significance of NMDAR-AB in complex neuropsychiatric disease versus health, however, have remained unclear. We tested sera of 2817 subjects (1325 healthy, 1081 schizophrenic, 263 Parkinson and 148 affective-disorder subjects) for presence of NMDAR-AB, conducted a genome-wide genetic association study, comparing $A B$ carriers versus non-carriers, and assessed their influenza $A B$ status. For mechanistic insight and documentation of $A B$ functionality, in vivo experiments involving mice with deficient bloodbrain barrier $\left(\mathrm{ApoE}^{-/-}\right)$and in vitro endocytosis assays in primary cortical neurons were performed. In 10.5\% of subjects, NMDAR$A B$ (NR1 subunit) of any immunoglobulin isotype were detected, with no difference in seroprevalence, titer or in vitro functionality between patients and healthy controls. Administration of extracted human serum to mice influenced basal and MK-801-induced activity in the open field only in $\mathrm{ApoE}^{-1-}$ mice injected with NMDAR-AB-positive serum but not in respective controls. Seropositive schizophrenic patients with a history of neurotrauma or birth complications, indicating an at least temporarily compromised bloodbrain barrier, had more neurological abnormalities than seronegative patients with comparable history. A common genetic variant ( $r 5524991, P=6.15 \mathrm{E}-08)$ as well as past influenza $\mathrm{A}(P=0.024)$ or $\mathrm{B}(P=0.006)$ infection were identified as predisposing factors for NMDAR-AB seropositivity. The $>10 \%$ overall seroprevalence of NMDAR-AB of both healthy individuals and patients is unexpectedly high. Clinical significance, however, apparently depends on association with past or present perturbations of blood-brain barrier function.

Molecular Psychiatry (2013) 0, 000-000. doi:10.1038/mp.2013.110

Keywords:

\section{INTRODUCTION}

$\mathrm{N}$-methyl-D-aspartate receptors (NMDAR) are glutamate-gated ion channels, abundantly expressed in mammalian brain. ${ }^{1}$ They form heteromers of NR1, NR2 and NR3 subunits, and are pivotal in regulating synapse function. ${ }^{2}$ In schizophrenia, NMDAR hypofunction has been hypothesized due to induction of psychotic symptoms by antagonists. ${ }^{3}$ In 2007, Dalmau et al. ${ }^{4,5}$ described a paraneoplastic syndrome, based on 12 women with ovarian teratoma, carrying lgG autoantibodies (AB) against the NMDAR NR1/2 subunits. The syndrome, termed 'anti-NMDAR encephalitis', variably consisted of psychosis, memory deficits, seizures, dyskinesia, decreased consciousness and autonomic instability. Since its initial description, a flood of publications appeared. The search for anti-NR1 IgG $A B$ in small samples $(N=46-80)$ of schizophrenic patients yielded discordant results. ${ }^{6-8}$ Recently, >400 previously collected cases of anti-NMDAR encephalitis have been reviewed, most without associated tumor. ${ }^{9}$ Similarly, immunomodulatory treatment outcomes of these and around 100 more cases have been summarized. ${ }^{10}$ As a syndromepertinent pathophysiological mechanism, an AB-induced decrease of NMDAR-mediated currents, due to enhanced receptor internalization and thus reduced surface expression, has been suggested. ${ }^{11}$

Few studies explored a role of other classes of immunoglobulins (lg) in an NMDAR-AB syndrome. In individuals with slow cognitive impairment, anti-NR1 IgA AB were found, which affected synaptic protein expression and decreased NMDAR-mediated currents. ${ }^{12}$ Anti-NR1 IgM $A B$ were described in a patient with bipolar disorder ${ }^{13}$ and in patients with herpes simplex encephalitis. ${ }^{14}$ In the largest study so far, investigating $\lg G$, $\lg A$ and $\lg M$, Steiner et $a l^{15}$ reported a higher prevalence of $A B$ of all isotypes in 121 schizophrenic patients, compared with healthy controls or patients suffering from affective disorders. Apart from tumors, no sound information is available yet on putative susceptibility factors for the development of anti-NR1 AB.

The present study was designed to (1) systematically screen in an unbiased fashion a large number $(N=2817)$ of healthy

${ }^{1}$ Clinical Neuroscience, Max Planck Institute of Experimental Medicine, Göttingen, Germany; ${ }^{2}$ Department of Psychiatry \& Psychotherapy, University Medicine Göttingen, Göttingen, Germany; ${ }^{3}$ DFG Research Center Nanoscale Microscopy and Molecular Physiology of the Brain (CNMPB), Göttingen, Germany; ${ }^{4}$ German Center for Neurodegenerative Diseases (DZNE), Göttingen, Germany; ${ }^{5}$ Department of Neurosurgery, University Clinic of Würzburg, Würzburg, Germany; ${ }^{6}$ Department of Molecular Biology of Neuronal Signals, Max Planck Institute of Experimental Medicine, Göttingen, Germany; ${ }^{7}$ Department of Neuroimmunology, Institute for Multiple Sclerosis Research and Hertie Foundation, University Medicine Göttingen, Göttingen, Germany; ${ }^{8}$ Institute for Experimental Immunology, affiliated to Euroimmun, Lübeck, Germany; ${ }^{9}$ Synaptic Systems GmbH, Göttingen, Germany; ${ }^{10}$ Institute of Virology, Hannover Medical School, Hannover, Germany; ${ }^{11}$ Department of Neurogenetics, Max Planck Institute of Experimental Medicine, Göttingen, Germany and ${ }^{12}$ Department of Clinical and Behavioral Neurology, IRCCS Santa Lucia Foundation, Rome, Italy. Correspondence: Professor H Ehrenreich, Clinical Neuroscience, Max Planck Institute of Experimental Medicine, Göttingen 37075, Germany. 
individuals and subjects suffering from schizophrenia or other brain diseases for presence of NMDAR-AB of $\lg G$, IgA or $\lg M$ isotype; (2) specifically address the question of why healthy $A B$ carriers might remain healthy, by extending this work to experiments in vivo and in vitro; (3) search for genetic and environmental factors predisposing to NMDAR-AB formation.

\section{MATERIALS AND METHODS}

\section{Participants}

Subject data were collected in accordance with ethical guidelines and the Declaration of Helsinki. Sample selection was unbiased, that is sera collection concluded before analysis of NMDAR-AB was planned. Schizophrenic patients $(N=1081)$ were recruited in 2005-2011 at 23 German sites in the GRAS (Göttingen Research Association for Schizophrenia) study. Patients fulfilling DSM-IV criteria for schizophrenia (81.5\%) or schizoaffective disorder (18.5\%) were included regardless of disease stage. ${ }^{16,17}$ Healthy GRAS controls were anonymized blood donors $(N=1272$ Transfusion Medicine, Göttingen). Health was ensured by pre-donation screening (questionnaires, interviews, hemoglobin, blood pressure, pulse, temperature). Patients with affective disorders $(N=148)$ were also included (ongoing GRAS extension). Parkinson patients $(N=263)$ and respective controls $(N=53)$ were recruited in 2010-2011 in Italy (Rome area). Of the GRAS patients, three volunteers carrying high titers of antiNR1 IgG, IgA, or IgM, and three seronegative controls agreed to blood donation for mouse experiments (Supplementary Table S1, Supplementary Appendix).

\section{Phenotypical analyses}

On all schizophrenic (GRAS) patients, extensive phenotypical characterization was performed as referenced previously. ${ }^{16,17}$ Age of onset, age at first psychotic episode, positive and negative syndrome scale scores, chlorpromazine equivalents, neurological symptoms (CNI; Cambridge Neurological Inventory) including fine motor skills (MacQuarrie dotting/ tapping), current cognitive functioning (composite score comprising reasoning, executive function, verbal learning and memory), premorbid intelligence and global assessment of functioning were employed as disease characteristics. As factors affecting blood-brain barrier (BBB) integrity, past neurotrauma (all severity levels) and birth complications (all pre- and perinatal complications) were carefully and comprehensively assessed. The final judgment regarding experience of birth complications or neurotrauma in the schizophrenic (GRAS) patient cohort was derived from a number of different sources. First, the information from semistructured interviews about birth and neurotrauma history of each patient was used. To verify the data or increase the amount of detailed information, all discharge letters of each single patient were screened. In the case of neurotrauma, other semi-structured interviews on critical life events, suicidality and aggressive behavior toward others were used to explore whether patients had experienced serious accidents (including brain trauma) or committed suicide attempts that included, for example, falls or jumps from great heights or had been involved in serious fights leading to head injuries. Finally, information from the physical exam of each patient was included to check whether any scars on the head or neck were found indicative of an injury to the head. After collecting all the data, each patient was dichotomously (yes/no) classified as having or not having experienced a neurotrauma or birth complication. In case of contradictory information, the treating physician and even the obstetric hospital were contacted, and in case of still missing information or a high level of uncertainty, patients were excluded from the analysis.

\section{Serological analyses}

Commercially available recombinant immunofluorescence tests (Euroimmun, Lübeck, Germany), standard procedures for clinical diagnosis $(100 \%$ sensitivity and $100 \%$ specificity), were used to detect NMDAR-AB, based on HEK293 cells transfected with NR1 or NR1/NR2b NMDAR-subunits. ${ }^{5,18}$ Seropositivity was assessed by two researchers independently. Titers were double-determined in two laboratories (MPI, Euroimmun) by identifying the maximum dilution at which specific fluorescence was still visible. Few samples with discrepant results were re-analyzed, leading to full concordance. The presence of $\lg G A B$ against influenza $A$ and $B$ virus was determined by ELISA (Novagnost-InfluenzaA-lgG, NovagnostInfluenzaB-IgG, Siemens Healthcare Diagnostics GmbH, Eschborn, Germany), automatically processed on BEPIII (Siemens Healthcare
Diagnostics $\mathrm{GmbH}$ ), and interpreted (manufacturer's instructions) as positive, negative or borderline (the latter negative for statistics).

\section{Immunoglobulin purification}

Ammonium sulfate precipitation of a serum fraction containing immunoglobulins (Ig) and dialysis was carried out as described. ${ }^{19} \operatorname{lgG}$, IgA, or $\lg M$ were quantified by immunodiffusion using NOR partigen immunoplates (Siemens Medical Solutions, Marburg, Germany).

\section{Mouse experiments}

Experiments were approved by the local Animal Protection Committee. Male C57BI/6N ApoE $-1-20$ and wild-type (WT) mice, aged 12-16 weeks, were used (housed at 4-5 per cage, $12 \mathrm{~h}$ light/dark cycle, food/water ad libitum). Groups (4-6 each) received either extracted lg fractions from NMDAR-AB seropositive (IgG, $\lg A$, or $\lg M$ ) or seronegative individuals (information on titer/concentration in Supplementary Table S1 and Supplementary Appendix). Daily intravenous (tail) injections (150 $\mu \mathrm{l}$ each) were performed on 3 consecutive days. Examiners were not aware of group assignment ('blinded'). Spontaneous activity in open field (8 min) was tested in all mice 3-4 days before the first injection (initial group matching). One day after the last injection, spontaneous activity ( $8 \mathrm{~min})$ was again measured, followed by intraperitoneal injection $(0.3 \mu \mathrm{g}$ per $10 \mu \mathrm{l}$ per gram of body weight) of the non-competitive NMDAR antagonist MK801 (Dizocilpine; [5R,10S]-(+)-5-methyl-10,11-dihydro-5H-dibenzo[a,d]cyclohepten-5,10-imine; Sigma-Aldrich $\mathrm{GmbH}$, Munich, Germany) and extended (120 min) open-field observation. ${ }^{21}$ MK-801 acts as usedependent ion-channel blocker.

\section{Endocytosis assays and quantification}

Primary cortical neurons prepared from mouse embryos (E16) were cultured on poly-L-lysine-coated glass-coverslips in MEM + B27 (Invitrogen, Karlsruhe, Germany) for 14 days. Glass coverslips were washed with cold Hank's balanced salt solution (HBSS), and incubated $\left(20 \mathrm{~min}, 4^{\circ} \mathrm{C}\right)$ with $\mathrm{lg}$ extracts containing either NMDAR-AB of $\lg G$, IgA or $\lg M$ isoforms or seronegative Ig extracts $(1: 100)$ in HBSS. The examiner was unaware of the nature of the extract ('blinded'). Unbound AB was removed (3 HBSS washes) before placing cells in pre-warmed $\left(37^{\circ} \mathrm{C}\right)$ growth medium for $15 \mathrm{~min}$ to allow internalization. After medium wash-off (cold HBSS), remaining surface NMDAR were labeled with anti-mouse NR1-AB (Abcam, Cambridge, UK) for $15 \mathrm{~min}$ on ice. After cold HBSS wash, surface-bound $\mathrm{NR} 1-\mathrm{AB}$ was labeled $\left(4^{\circ} \mathrm{C}, 15 \mathrm{~min}\right)$ with Alexa-488-coupled 2nd $\mathrm{AB}$ (antimouse IgG; Invitrogen, Karlsruhe, Germany). After wash-off (HBSS; $4^{\circ} \mathrm{C}$ ) of unbound $A B$, cells were fixed in $4 \%$ paraformaldehyde. For quantification, confocal images of cell surface staining were taken with identical acquisition parameter on SP2 LSM (Leica, Wetzlar, Germany). Signal intensity was quantified with ImageJ, and ratio of intensity per cell surface area calculated.

\section{Genetic analyses}

A semi-custom Axiom myDesign genotyping array (Affymetrix, Santa Clara, CA, USA) was used. For description of array-specifications, quality controls and genome-wide genetic association study (GWAS), see Supplementary Appendix.

\section{Statistical analysis}

$P$-values $<0.05$ were considered significant. Data in figures are expressed as mean \pm s.e.m., in tables as mean \pm s.d.

Mouse experiments. Data were compared by analysis of variance, followed by post-hoc tests where appropriate using Prism5 (GraphPad-Software Inc., La Jolla, CA, USA) or SPSS (SPSS-Statistics 17.0, IBM-Deutschland GmbH, Munich, Germany). Greenhouse-Geisser correction was applied on violation of sphericity.

Human data. Group differences in categorical and continuous variables were assessed using Chi-square or Mann-Whitney $\mathrm{U}$ tests. A generalized linear model was employed upon covariate inclusion. At normal distribution of continuous variables, $T$-tests were performed. To assess main effects and interactions between neurotrauma/birth complications and Ig-positivity regarding CNI scores, two-way independent ANOVA was conducted. Corrected values reflect linear regression-based residuals when 
Table 1. Prevalence of anti-NR1 AB in patients and controls

Anti-NR1 seropositivity-N (\%)

\begin{tabular}{|c|c|c|c|c|c|c|c|c|}
\hline Study group & Any & $\lg G$ & $\lg A$ & $\lg M$ & $\lg G+\lg A$ & $\lg G+\lg M$ & $\lg A+\lg M$ & $\lg G+\lg A+\lg M$ \\
\hline GRAS $^{\text {a }}$ patients $(N=1081)$ & $93(8.6)$ & $7(0.7)$ & $56(5.2)$ & $46(4.3)$ & $0(0)$ & $0(0)$ & $16(1.5)$ & $0(0)$ \\
\hline Affective-disorder patients $(N=148)$ & $24(16.2)$ & $5(3.4)$ & $15(10.1)$ & $7(4.7)$ & $1(0.7)$ & $0(0)$ & $2(1.4)$ & $0(0)$ \\
\hline GRAS $^{\mathrm{a}}$ controls $(N=1272)$ & $137(10.8)$ & $5(0.4)$ & $75(5.9)$ & $80(6.3)$ & $2(0.2)$ & $3(0.2)$ & $19(1.5)$ & $1(0.1)$ \\
\hline Parkinson patients $(N=263)$ & $35(13.3)$ & $1(0.4)$ & $17(6.5)$ & $25(9.5)$ & $1(0.4)$ & $1(0.4)$ & $7(2.7)$ & $1(0.4)$ \\
\hline Parkinson controls $(N=53)$ & $6(11.3)$ & $0(0)$ & $3(5.7)$ & $3(5.7)$ & $0(0)$ & $0(0)$ & $0(0)$ & $0(0)$ \\
\hline Total $(N=2817)$ & $295(10.5)$ & $18(0.6)$ & $166(5.9)$ & $161(5.7)$ & $4(0.1)$ & $4(0.1)$ & $44(1.6)$ & $2(0.1)$ \\
\hline
\end{tabular}

${ }^{a}$ GRAS patients are schizophrenic individuals of the GRAS data collection (Göttingen Research Association for Schizophrenia). GRAS controls are the respective healthy control collective (see also Materials and methods).

age, chlorpromazine and positive and negative syndrome scale negative scores were independent variables. PLINK (v1.07) was used to test association between single-nucleotide polymorphisms (SNPs) and antiNR1 serological status (allelic test) and deviations from Hardy-Weinberg equilibrium. ${ }^{22}$ Principal components were generated using EIGENSTRAT (http://genepath.org. harvard.edu/ reich/EIGENSTRAT.htm). Human leukocyte antigen (HLA) types were imputed for seven HLA genes using HiBag0.9.1 at four-digit resolution, based on a pre-fit European ancestry model (http://students.washington.edu/zhengx/HIBAG/). P-values were multiple-testing corrected (Bonferroni) where indicated, but are displayed uncorrected.

\section{RESULTS}

NMDAR-AB seroprevalence in 2817 individuals

$A B$ of all here analyzed isotypes ( $\lg G, \lg A$ and $\lg M)$, directed against the NMDAR-NR1 subunit, were identified in $10.5 \%$ of subjects (Table 1). Importantly, seroprevalence did not differ between schizophrenic (GRAS) patients (8.6\%) and GRAS controls $(10.8 \%, P=0.078)$. An apparently higher incidence in affectivedisorder patients (16.2\%) is explained by a higher mean age. In fact, seroprevalence increases with age (Supplementary Figure S1, Supplementary Table S2, Supplementary Appendix) and is higher in male than female subjects (Supplementary Table S3, Supplementary Appendix, $11.53 \%$ versus $8.68 \%, P=0.017)$. Seropositivity between Parkinson patients (13.3\%) and respective controls did not differ $(11.3 \%, P=0.694)$.

Seroprevalence and titer distribution of NMDAR-AB lg isotypes Considering each Ig class separately, again no differences in seroprevalence among groups arose (Table 1). Occurrence of IgG anti-NR1 was infrequent (0.6\% in total) compared with $\operatorname{lgA}(5.9 \%)$ or $\lg M(5.7 \%)$. A combination of $\lg A / \lg M A B$ was present in $1.6 \%$, combinations including lgG in only $0.1 \%$ each. $A B$ exclusively against the NR1/NR2b heterodimer, that is without reactivity against NR1 alone, were not identified. Titer distributions in patient and control groups as possible explanation of NMDAR-AB pathology did not differ (Supplementary Table S4, Supplementary Figure S2, Supplementary Appendix).

\section{NMDAR-AB functionality in a neuronal endocytosis assay}

We next wondered whether $A B$ from controls and patients differ in functionality. Extracts from seropositive subjects, independently of isotype or disease state, resulted in increased endocytosis, compared with seronegative extracts (Figure 1a, Supplementary Table S1, Supplementary Appendix).

Relevance of BBB integrity for NMDAR-AB effects in mice

Having comparable serological (\% seropositivity, Ig-isotype, titer distribution) and functional results in controls and patients, we asked why healthy $A B$ carriers remain healthy. We hypothesized that a compromised $B B B$ might decide on the pathophysiological significance of NMDAR-AB. To approach this hypothesis experimentally, we employed $\mathrm{ApoE}^{-1-}$ mice $^{20}$ (with known BBB leakage $)^{23-25}$ versus WT. Intravenous injection of purified Ig fractions from NMDAR-AB seropositive (IgM, IgG, IgA) subjects led to alterations in spontaneous open-field activity and the response to $\mathrm{MK}-801$ exclusively in $\mathrm{ApoE}^{-1-}$ mice. Trends were comparable in groups receiving $\operatorname{lgM}$, IgG or IgA extracts, resulting in significant differences on pooling (Figures $1 \mathrm{~b}$ and $\mathrm{C}$, Supplementary Figure S3, Supplementary Appendix).

\section{Translating experimental BBB findings to schizophrenic (GRAS)} patients

Overall, schizophrenic anti-NR1 carriers and non-carriers do not differ with respect to disease phenotypes, covering the symptom clusters of anti-NMDAR encephalitis (Table 2). Also, occurrence and duration of prodromal phase and age of disease onset are similar between the two groups, arguing against a sudden/ atypical syndrome start in AB carriers (Table 2). Following our BBB hypothesis, we compared individuals with birth complications or past brain trauma-conditions known to provoke temporary or persistent (albeit often minor) BBB abnormalities. ${ }^{26,27}$ Indeed, also in humans, a clear impact, that is a more severe neurological phenotype, arises from the combination of compromised BBB function and circulating NMDAR-AB (Figure 1d).

Identification of first genetic susceptibility factors

GWAS have been successful in identifying associations between genomic variants and autoimmune disorders, such as rheumatoid arthritis or systemic lupus erythematosus. ${ }^{28}$ We performed GWAS to spot SNPs potentially predisposing to formation of NMDAR-AB (Supplementary Appendix). We identified a genomewide significant SNP, rs524991 (A/G, $P=6.15 \mathrm{E}-08$; Bonferroni threshold $P=8.62 \mathrm{E}-08$ ), with an odds ratio (OR) of 2.22 (95\% confidence interval $(\mathrm{Cl})=1.654-2.991$; Supplementary Figure S6, Supplementary Appendix). This variant with a minor allele frequency of $12.45 \%$ in seropositive versus $6.01 \%$ in seronegative individuals is located in an intergenic region on chromosome1 (Supplementary Figure S7, Supplementary Appendix). Its closest neighboring gene is nuclear factor I/A (NFIA, $218.59 \mathrm{~kb}$ downstream), a transcription factor reported to mediate neuroprotective effects of NMDAR activation. ${ }^{29}$ Separate analysis of SNP rs524991 association with NMDARAB seropositivity (Table 3 ) showed a similar tendency for all study groups (except Parkinson) and no gender difference (Supplementary Table S8, Supplementary Appendix). Search for a predisposing role of HLA alleles for NMDAR-AB formation did not deliver hits, apart from a nominally significant association 


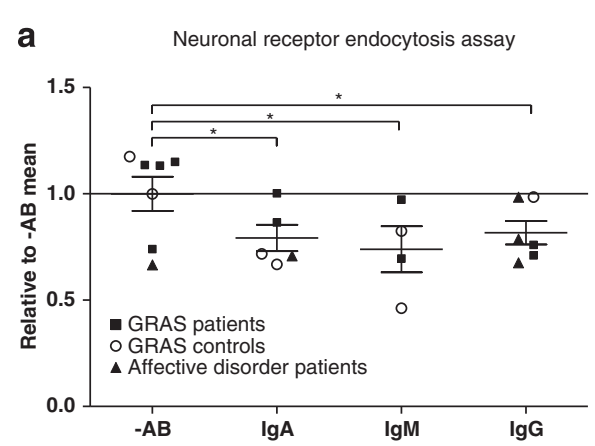

b

Mice: Spontaneous activity in open field Before $A B$ application

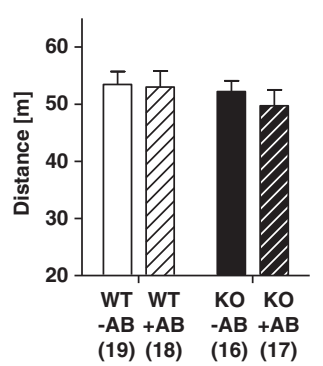

After $\mathrm{AB}$ application

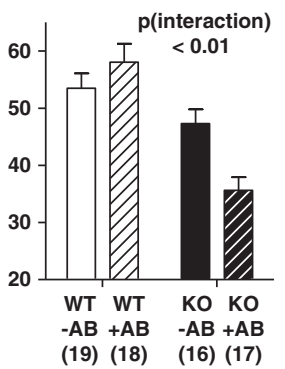

c

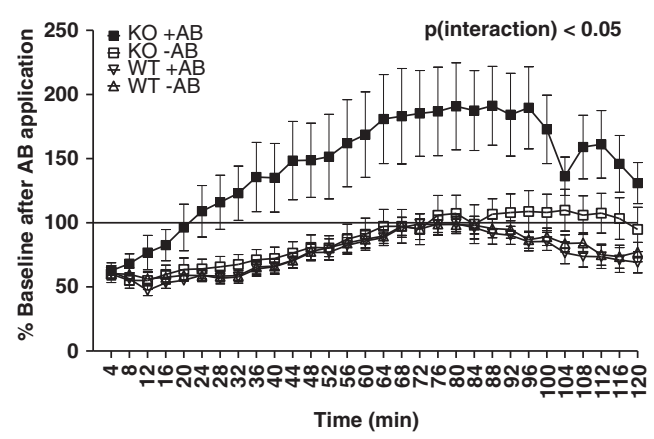

d

GRAS patients: BBB challenges \& AB status

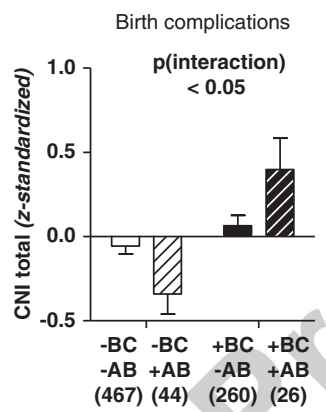

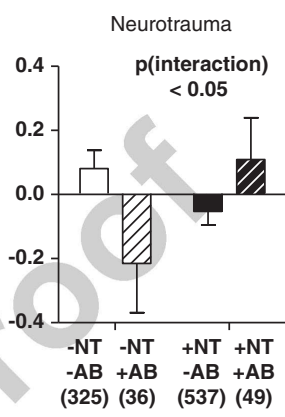

Figure1. NMDAR-AB functionality and relevance of the blood-brain barrier. (a) Reduced $A B$ binding to primary cortical neurons indicates increased endocytosis of NMDAR after incubation with lg extracts containing either NMDAR-AB of $\lg$, $\lg A$ or $\lg M$ isoforms (all $P<0.05$ ), or seronegative Ig extracts (one-tailed $T$-tests). Mean values upon AB extracts (each tested in 1-3 independent experiments, dependent on serum availability) were normalized to the mean of the respective seronegative control extracts. (b) ApoE ${ }^{-1-}$ (KO) and WT mice do not differ in spontaneous activity in the open field before $A B$ injection. However, 1 day after the last of three daily injections with seropositive ( $+A B$ ) or seronegative $(-\mathrm{AB})$ Ig extracts, a decrease in spontaneous activity was evident exclusively in seropositive $\mathrm{ApoE}{ }^{-1-}(\mathrm{KO}+\mathrm{AB}) \mathrm{mice}$ : two-way ANOVA revealed a significant interaction effect of genotype and serotype $(F=8.96, P<0.01)$, as well as a significant main effect of genotype $(F=27.81, P<0.0001)$, but not of serotype. (c) Only $\mathrm{ApoE}^{-\prime-}(\mathrm{KO})$ mice with their known compromised BBB respond to intravenous NMDAR$A B$ extracts with a hypersensitive (psychosis-related) response in the open field to the NMDAR antagonist MK-801. Using a generalized linear model for repeated measures to evaluate results after MK-801 treatment, we obtained significant main effects of time $(F=36.25, P<0.001)$, genotype $(F=9.54, P<0.01)$ and serotype $(F=4.85, P<0.05)$ as well as a significant genotype $\times$ serotype interaction effect $(F=5.75, P<0.05)$. (d) In the GRAS sample of schizophrenic patients, birth complications and history of neurotrauma as readouts for temporarily/persistently compromised BBB were examined in NMDAR-AB-positive versus negative subjects with respect to their impact on neurological symptom severity (CNI total score, $z$-standardized). Birth complications showed an interaction with serotype $(F=5.80, P<0.05)$ regarding $C N \mathrm{NI}$ and a main effect $(F=11.24, P=0.001)$. Likewise, past neurotrauma showed an interaction with serotype $(F=4.02, P<0.05)$. Group sizes are given as numbers in brackets. ${ }^{*} P<0.05$; WT, wild type; $\mathrm{KO}, \mathrm{ApoE}^{-/-}$knockout; $-\mathrm{AB}$, NMDAR-AB seronegative; $+\mathrm{AB}, \mathrm{NMDAR-AB}$ seropositive; $\mathrm{BC}$, birth complications; NT, neurotrauma.

of HLA-A03 with seropositivity $(P=0.01$; Supplementary Table S9, Supplementary Appendix).

Identification of environmental susceptibility factors

As first risk factor for NMDAR-AB formation, the presence of a tumor, preferentially an ovarian teratoma was identified. ${ }^{4}$ Other predisposing factors have remained speculative. Infections have been suggested as triggers of $A B$ formation in autoimmune diseases, ${ }^{30}$ for example, Epstein-Barr virus in multiple sclerosis. ${ }^{31}$ We hypothesized that a similar role might be attributed to influenza for NMDAR-AB. Anti-NMDAR encephalitis was reported in a patient with influenza H1N1 infection and two subjects after respective vaccination. ${ }^{9}$ NMDAR-AB were described in pediatric cases of encephalitis lethargica, a condition-not unequivocally-
associated with influenza. ${ }^{32,33}$ Strikingly, we found an increased anti-NR1 $A B$ prevalence in individuals carrying anti-influenza $A$ $(P=0.024, \mathrm{OR}=1.366, \mathrm{Cl} 95 \%=1.042-1.790)$ and $\mathrm{B}(P=0.006$, $\mathrm{OR}=1.453, \mathrm{Cl} 95 \%=1.109-1.904) \mathrm{lgG}$ (Table 3). This association was present in males only (Supplementary Table S10, Supplementary Appendix).

\section{DISCUSSION}

The present study provides (1) the first large-scale systematic screen for presence of NMDAR-AB in serum of healthy and neuropsychiatrically diseased subjects. In $>2800$ unbiasedly selected individuals, $>10 \%$ seroprevalence of anti-NR1 AB, independent of group affiliation, was found. (2) From this unexpected observation, the fundamental question arose of why healthy $A B$ carriers have remained healthy, despite comparable distribution of $A B$ isotypes, titers and in vitro functionality. An experimental mouse model supports our central hypothesis, that is, the essential role of BBB integrity. Only in ApoE mutant mice, but not in respective controls, we find that human NMDAR-AB cause psychosis-related behavioral perturbation. ${ }^{21}$ The BBB role is further underlined by a hypothesisdriven outcome analysis of schizophrenic (GRAS) patients with history of birth complications or neurotrauma indicating past/ present BBB insufficiency. (3) Ultimately, with a genome-wide significant marker, SNP rs524991, and an association of seropositivity with influenza $A B$ status, we provide genetic and environmental risk factors of NMDAR-AB formation.

The most remarkable finding of the present work is the high seroprevalence of NMDAR-AB in healthy individuals. Only one 
Table 2. Baseline characteristics, according to study group

\begin{tabular}{|c|c|c|c|c|}
\hline Patients and control groups & Total sample & Ig-positive individuals & Ig-negative individuals & $\begin{array}{c}\text { P-value }\left(\chi^{2}, Z,\right. \\
\text { T value })^{\mathrm{a}}\end{array}$ \\
\hline Schizophrenic (GRAS) patients & $\mathrm{N}=774-1081^{\mathrm{b}}$ & $\mathrm{N}=63-93^{\mathrm{b}}$ & $\mathrm{N}=711-988^{\mathrm{b}}$ & \\
\hline Age, years, mean \pm s.d., (range) & $39.37 \pm 12.59(17-79)$ & $41.15 \pm 11.98(18-75)$ & $39.20 \pm 12.63(17-79)$ & $\begin{array}{c}0.115 \\
(Z=-1.58)\end{array}$ \\
\hline Gender, $N(\%)$ & $723(66.9)$ & $68(73.1)$ & $655(66.3)$ & $\begin{array}{c}0.181 \\
\left(\chi^{2}=1.79\right)\end{array}$ \\
\hline Prodrome, $N$ cases $(\%)$ & $754(80.6)$ & $64(79.0)$ & $690(80.8)$ & $\begin{array}{c}0.698 \\
\left(\chi^{2}=0.15\right)\end{array}$ \\
\hline $\begin{array}{l}\text { Duration of prodrome, years, } \\
\text { mean } \pm \text { s.d., (range) }\end{array}$ & $2.81 \pm 3.57(0-28.2)$ & $2.64 \pm 3.01(0-13.0)$ & $2.83 \pm 3.62(0-28.2)$ & $\begin{array}{c}0.853 \\
(Z=-0.19)\end{array}$ \\
\hline $\begin{array}{l}\text { Age at first episode, years, } \\
\text { mean } \pm \text { s.d., (range) }\end{array}$ & $25.88 \pm 8.90(5-68)$ & $25.85 \pm 8.98(12-51)$ & $25.89 \pm 8.90(5-68)$ & $\begin{array}{c}0.890 \\
(Z=-0.11)\end{array}$ \\
\hline $\begin{array}{l}\text { PANSS positive score, mean } \pm \text { s.d., } \\
\text { (range) }\end{array}$ & $13.74 \pm 6.25(7-38)$ & $13.28 \pm 5.71(7-31)$ & $13.78 \pm 6.31(7-38)$ & $\begin{array}{c}0.597 \\
(Z=0.60)\end{array}$ \\
\hline $\begin{array}{l}\text { PANSS negative score, mean } \pm \text { s.d., } \\
\text { (range) }\end{array}$ & $18.25 \pm 7.90(7-46)$ & $17.00 \pm 7.42(7-37)$ & $18.37 \pm 7.93(7-46)$ & $\begin{array}{c}0.122 \\
(Z=-1.55)\end{array}$ \\
\hline $\begin{array}{l}\text { PANSS general score, mean } \pm \text { s.d., } \\
\text { (range) }\end{array}$ & $33.74 \pm 11.76(16-82)$ & $32.48 \pm 10.80(16-75)$ & $33.86 \pm 11.85(16-82)$ & $\begin{array}{c}0.373 \\
(Z=-0.89)\end{array}$ \\
\hline CPZ, mean \pm s.d., (range) & $686.53 \pm 697.43(0-7375)$ & $628.04 \pm 537.82(0-2620)$ & $691.97 \pm 710.43(0-7375)$ & $\begin{array}{c}0.580 \\
(Z=-0.55)\end{array}$ \\
\hline $\begin{array}{l}\text { Cambridge Neurological } \\
\text { Inventory }{ }^{c_{\text {,nd }}}, \text { mean } \pm \text { s.d., (range) }\end{array}$ & $0.00 \pm 1.00(-2.71-3.07)$ & $-0.03 \pm 0.92(-2.07-2.11)$ & $0.00 \pm 1.01(-2.71-3.07)$ & $\begin{array}{c}0.742 \\
(T=0.33)\end{array}$ \\
\hline $\begin{array}{l}\text { MacQuarrie dotting }{ }^{e} \text {, mean } \pm \text { s.d., } \\
\text { (range) }\end{array}$ & $0.00 \pm 1.00(-3.61-3.22)$ & $0.14 \pm 1.14(-2.67-3.07)$ & $-0.01 \pm 0.98(-3.61-3.22)$ & $\begin{array}{c}0.172 \\
(T=-1.37)\end{array}$ \\
\hline $\begin{array}{l}\text { MacQuarrie tapping }{ }^{\text {e, }} \text { mean } \pm \text { s.d., } \\
\text { (range) }\end{array}$ & $0.00 \pm 1.00(-4.83-3.14)$ & $0.11 \pm 0.99(-2.00-3.10)$ & $-0.01 \pm 1.00(-4.83-3.14)$ & $\begin{array}{c}0.261 \\
(T=-1.13)\end{array}$ \\
\hline $\begin{array}{l}\text { Cognitive composite score, } \\
\text { mean } \pm \text { s.d., (range) }\end{array}$ & $-0.02 \pm 0.84(-2.57-2.98)$ & $-0.01 \pm 0.89(-2.12-2.03)$ & $-0.02 \pm 0.84(-2.57-2.98)$ & $\begin{array}{c}0.946 \\
(T=-0.07)\end{array}$ \\
\hline $\begin{array}{l}\text { Premorbid IQ (MWT-B }{ }^{\mathrm{f}} \text { ), } \\
\text { mean } \pm \text { s.d., (range) }\end{array}$ & $25.67 \pm 6.36(4-42)$ & $26.64 \pm 6.28(6-36)$ & $5.57 \pm 6.36(4-42)$ & $\begin{array}{c}0.093 \\
(Z=-1.68)\end{array}$ \\
\hline $\begin{array}{l}\text { Global assessment of functioning, } \\
\text { mean } \pm \text { s.d., (range) }\end{array}$ & $45.70 \pm 17.18(5-90)$ & $46.26 \pm 16.54(10-80)$ & $45.65 \pm 17.25(5-90)$ & $\begin{array}{c}0.642 \\
(Z=-0.47)\end{array}$ \\
\hline Neurotrauma, $N$ cases (\%) & $648(62.4)$ & $55(62.6)$ & $593(59.8)$ & $\begin{array}{c}0.592 \\
\left(\chi^{2}=0.29\right)\end{array}$ \\
\hline Birth complications, $N$ cases (\%) & $307(39.7)$ & 27 (42.9) & $280(39.4)$ & $\begin{array}{c}0.589 \\
\left(\chi^{2}=0.29\right)\end{array}$ \\
\hline Healthy (GRAS) controls & $\mathrm{N}=1272$ & $\mathrm{~N}=137$ & $\mathrm{~N}=1135$ & \\
\hline Age, years, mean \pm s.d., (range) & $37.43 \pm 13.24(18-69)$ & $40.90 \pm 12.17(19-68)$ & $37.01 \pm 13.31(18-69)$ & $\begin{array}{c}<\mathbf{0 . 0 0 1} \\
(Z=-3.56)\end{array}$ \\
\hline Gender, $N$, male $(\%)$ & $780(61.3)$ & $95(69.3)$ & $685(60.4)$ & $\begin{array}{c}\mathbf{0 . 0 4 1} \\
\left(\chi^{2}=4.17\right)\end{array}$ \\
\hline Affective-disorder patients & $N=148$ & $N=24$ & $N=124$ & \\
\hline Age, years, mean \pm s.d., (range) & $49.70 \pm 15.49(20-92)$ & $47.38 \pm 11.87(25-76)$ & $50.15 \pm 16.09(20-92)$ & $\begin{array}{c}0.314 \\
(Z=-1.01)\end{array}$ \\
\hline Gender, $N$ male (\%) & $70(47.3)$ & $11(45.8)$ & 59 (47.6) & $\begin{array}{c}0.875 \\
\left(\chi^{2}=0.03\right)\end{array}$ \\
\hline Parkinson patients & $\mathrm{N}=253-263^{\mathrm{b}}$ & $\mathrm{N}=33-35^{\mathrm{b}}$ & $\mathrm{N}=220-228^{\mathrm{b}}$ & \\
\hline Age, years, mean \pm s.d., (range) & $66.04 \pm 10.08(36-86)$ & $69.06 \pm 8.33(45-81)$ & $65.59 \pm 10.26(36-86)$ & $\begin{array}{c}0.055 \\
(Z=-1.92)\end{array}$ \\
\hline Gender, $N$ male (\%) & $175(66.5)$ & $28(80.0)$ & $147(64.5)$ & $\begin{array}{c}0.070 \\
\left(\chi^{2}=3.29\right)\end{array}$ \\
\hline Parkinson controls & $\mathrm{N}=51-53^{\mathrm{b}}$ & $\mathrm{N}=6^{\mathrm{b}}$ & $\mathrm{N}=45-47^{\mathrm{b}}$ & \\
\hline Age, years, mean \pm s.d., (range) & $63.31 \pm 11.68(22-80)$ & $67.50 \pm 12.58(44-78)$ & $62.76 \pm 11.59(22-80)$ & $\begin{array}{c}0.188 \\
(Z=-1.33)\end{array}$ \\
\hline Gender, $N$ male (\%) & $21(39.6)$ & $2(33.3)$ & $19(40.4)$ & $\begin{array}{c}0.738 \\
\left(\chi^{2}=0.11\right)\end{array}$ \\
\hline
\end{tabular}

Abbreviations: CPZ, chlorpromazine equivalents; GRAS, Göttingen Research Association for Schizophrenia; PANSS, positive and negative syndrome scale. Bolded values, $P<0.05$. ${ }^{a}$ For statistical methods, Mann-Whitney $U$ or $\chi^{2}$ tests and for normally distributed variables, $T$-tests were used. ${ }^{b} D u e$ to missing data, sample sizes vary. ${ }^{c}$ Mean value if more than 95 items were available. ${ }^{d}$ Corrected for age and CPZ. ${ }^{e}$ Corrected for age, PANSS negative and CPZ. ${ }^{f}$ MehrfachWortschatz-Intelligenz test (multiple choice vocabulary test).

other study included a considerable number of healthy subjects in a screen of psychiatric patients but reported seropositivity for only 1 in 240 controls $(0.4 \%)$ and 2 in $108(<2 \%)$ affective-disorder patients. ${ }^{15}$ Seroprevalence in schizophrenic patients (9.9\% of 121) was comparable to our study. Reasons for this discrepancy are unclear but perhaps related to the smaller number of controls and 
their younger age. ${ }^{15}$ Importantly, analytical materials/methods of both studies were identical, schizophrenia patients show comparable seroprevalence, and the here randomly selected positive specimens for in vitro analyses all confirmed $A B$ functionality.

For exerting pathological effects, NMDAR-AB have to reach NMDAR in the brain. This brain presence may occur via (1) $A B$ transfer over a compromised $\mathrm{BBB}$, which normally restricts large molecules from directly entering the brain in appreciable amounts (expected transfer over an intact BBB, for example, IgG is only 1/ 500 , of $\lg A 1 / 600$, and of $\lg M 1 / 3000$ of the serum concentration); (2) slow accumulation of these large molecules due to reduced cerebrospinal fluid (CSF) flow, $^{34}$ in which case, however, a retrograde CSF circulation would have to deliver the $A B$ back to brain tissue, or (3) intrathecal synthesis by B lymphocytes. ${ }^{34,35}$ Our seroprevalence data do not allow any conclusion on $A B$ production in brain. Therefore, healthy $A B$ carriers may differ at least in part from seropositive disease groups by lack of intrathecal $A B$ synthesis. Nevertheless, with in vivo experiments in mice and a hypothesis-driven human database screening, we underscore the likely critical role of an intact BBB as protective mechanism against circulating $A B-$ mediated pathology in mouse and man.

Wild-type mice were not behaviorally affected after injection of human serum extract containing IgG, IgA or IgM NMDAR-AB. In contrast, $A p o E^{-1-}$ mice showed differences in behavior on $A B$ injection, that is reduced spontaneous activity in the open field and hyperlocomotion following MK-801. These behavioral phenomena may be explained by the reported receptor internalization and hypofunction after hippocampal infusion of NMDAR-AB, ${ }^{11}$ as exactly opposite effects were described after NMDA application in rats. ${ }^{36}$ Stimulation of locomotion by the NMDAR antagonist MK-801 may be caused by hyperexcitability of limbic circuits through NMDAR blockade on inhibitory GABAergic neurons. ${ }^{37,38}$ This consequence of NMDA receptor inhibition would be amplified by NMDAR-AB. Similarly, increased motor cortex excitability in mice was provoked by NMDAR-AB injection into the prefrontal area. ${ }^{39}$

In the GRAS sample of schizophrenic individuals, well-documented history of birth complications and brain trauma were evaluated as proxy variables of past or present BBB impairment. ${ }^{26,27}$ Indeed, affected individuals show more severe neurological abnormalities when carrying NMDAR-AB. These findings strengthen the hypothesis of $B B B$ involvement in NMDAR-AB pathology, and-replication provided-may even justify recommendations of anti-NR1 serum screening in case of neurotrauma or other conditions with anticipated BBB dysfunction.

Importantly, we did not find any clinically relevant differences when comparing all schizophrenic NMDAR-AB carriers with all non-carriers. Perhaps with information on CSF (which we do not have in our large cohort), an expected $30 \%$ of individuals with permanent barrier dysfunction ${ }^{40}$ could have been extracted and would have allowed us to uncover a clinically relevant difference between $A B$ carriers and non-carriers among them. Instead, we found a clinical difference between $A B$ carriers and non-carriers with past birth complication or neurotrauma as a proxy for at least temporary BBB disturbance. ${ }^{26}$ It is interesting to speculate that the reported $30 \%$ of schizophrenic patients with compromised barrier function ${ }^{40}$ and the post-trauma individuals recognized here might represent an (partly) overlapping subpopulation of schizophrenic subjects. Along these lines of thought, future studies may be initiated, analyzing CSF samples of a large number of schizophrenic patients for NMDAR-AB titers and determining the CSF-serum albumin quotient ${ }^{34}$ as marker of blood-CSF barrier (dys)function.

Our study is the first to investigate putative genetic susceptibility factors for the formation of anti-NR1 AB. A GWAS approach led to the identification of the genome-wide significant risk SNP 
rs524991. Further experiments providing mechanistic insight as well as replication analyses are warranted. By a hypothesis-driven approach, ${ }^{9,32,33}$ we uncovered an association of influenza A or B $A B$ with anti-NR1 seropositivity, suggesting molecular mimicry. This phenomenon induces generation of $A B$ reacting both against pathogenic elements and autoantigens, ${ }^{30}$ and has a role in autoimmune diseases. ${ }^{41,42}$ As the influenza A M2 channel and NMDAR share a common ligand, the antiviral compound amantadine, $^{43}$ a putative structural homology might act as inducer of NMDAR-AB. The observed association was found in males only. Interestingly, males have a higher incidence of influenza, ${ }^{44}$ and male mice exert a more vigorous immune response on influenza infection. ${ }^{45}$ This gender disposition might also explain the higher anti-NR1 AB prevalence in males.

In conclusion, our study draws an increasingly complex picture of NMDAR-AB pathology, with anti-NMDAR encephalitis possibly constituting the extreme end of a broad spectrum of mild to severe phenotypes associated with NMDAR autoimmunity. Beyond the NMDAR-AB studied here, loss of blood-brain barrier integrity may generally constitute a major risk factor for detrimental effects of peripheral $A B$ against central nervous system epitopes.

\section{CONFLICT OF INTEREST}

Dr Stöcker is a full-time employee of and holds stocks in Euroimmun AG. Dr Martens is a full-time employee of Synaptic Systems $\mathrm{GmbH}$. All other authors declare no conflict of interest.

\section{ACKNOWLEDGEMENTS}

This work was supported by the Max Planck Society, the Max Planck Förderstiftung and the DFG (CNMPB). Christian Hammer acknowledges grant support by the Daimler and Benz Foundation as well as by the Brain and Behavior Foundation. Suhaidah Mohd Jofrry, through support by the Faculty of Pharmacy, Universiti Teknologi MARA, Bandar Puncak Alam, Selangor, Malaysia, received a scholarship from the Ministry of Higher Education Malaysia. We are grateful to Sylvia Siefarth, Euroimmun, for excellent technical assistance.

\section{REFERENCES}

1 Li F, Tsien JZ. Memory and the NMDA receptors. N Engl J Med 2009; 361: 302-303.

2 Lau CG, Zukin RS. NMDA receptor trafficking in synaptic plasticity and neuropsychiatric disorders. Nat Rev Neurosci 2007; 8: 413-426.

3 Homayoun $\mathrm{H}$, Moghaddam B. NMDA receptor hypofunction produces opposite effects on prefrontal cortex interneurons and pyramidal neurons. J Neurosci 2007; 27: $11496-11500$.

4 Dalmau J, Tuzun E, Wu HY, Masjuan J, Rossi JE, Voloschin A et al. Paraneoplastic anti-N-methyl-D-aspartate receptor encephalitis associated with ovarian teratoma. Ann Neurol 2007; 61: 25-36.

5 Dalmau J, Gleichman AJ, Hughes EG, Rossi JE, Peng X, Lai M et al. Anti-NMDAreceptor encephalitis: case series and analysis of the effects of antibodies. Lancet Neurol 2008; 7: 1091-1098.

6 Masdeu JC, Gonzalez-Pinto A, Matute C, Ruiz De Azua S, Palomino A, De Leon J et al. Serum IgG antibodies against the NR1 subunit of the NMDA receptor not detected in schizophrenia. Am J Psychiatry 2012; 169: 1120-1121.

7 Zandi MS, Irani SR, Lang B, Waters P, Jones PB, McKenna P et al. Disease-relevant autoantibodies in first episode schizophrenia. J Neurol 2011; 258: 686-688.

8 Tsutsui K, Kanbayashi T, Tanaka K, Boku S, Ito W, Tokunaga J et al. Anti-NMDAreceptor antibody detected in encephalitis, schizophrenia, and narcolepsy with psychotic features. BMC Psychiatry 2012; 12 : 37.

9 Dalmau J, Lancaster E, Martinez-Hernandez E, Rosenfeld MR, Balice-Gordon R. Clinical experience and laboratory investigations in patients with anti-NMDAR encephalitis. Lancet Neurol 2011; 10: 63-74.

10 Titulaer MJ, McCracken L, Gabilondo I, Armangue T, Glaser C, lizuka T et al. Treatment and prognostic factors for long-term outcome in patients with antiNMDA receptor encephalitis: an observational cohort study. Lancet Neurol 2013; 12: 157-165.

11 Hughes EG, Peng X, Gleichman AJ, Lai M, Zhou L, Tsou R et al. Cellular and synaptic mechanisms of anti-NMDA receptor encephalitis. J Neurosci 2010; 30: 5866-5875.
12 Pruss $\mathrm{H}$, Holtje M, Maier N, Gomez A, Buchert R, Harms L et al. IgA NMDA receptor antibodies are markers of synaptic immunity in slow cognitive impairment. Neurology 2012; 78: 1743-1753.

13 Choe CU, Karamatskos E, Schattling B, Leypoldt F, Liuzzi G, Gerloff C et al. A clinical and neurobiological case of IgM NMDA receptor antibody associated encephalitis mimicking bipolar disorder. Psychiatry Res 2012; 208: 194-196.

14 Pruss H, Finke C, Holtje M, Hofmann J, Klingbeil C, Probst C et al. N-methyl-Daspartate receptor antibodies in herpes simplex encephalitis. Ann Neurol 2012; 72: 902-911.

15 Steiner J, Walter M, Glanz W, Sarnyai Z, Bernstein HG, Vielhaber S et al. Increased prevalence of diverse $\mathrm{N}$-methyl-D-aspartate glutamate receptor antibodies in patients with an initial diagnosis of schizophrenia: specific relevance of IgG NR1a antibodies for distinction from N-methyl-D-aspartate glutamate receptor encephalitis. JAMA Psychiatry 2013; 70: 271-278.

16 Begemann M, Grube S, Papiol S, Malzahn D, Krampe H, Ribbe K et al. Modification of cognitive performance in schizophrenia by complexin 2 gene polymorphisms. Arch Gen Psychiatry 2010; 67: 879-888.

17 Ribbe K, Friedrichs H, Begemann M, Grube S, Papiol S, Kastner A et al. The crosssectional GRAS sample: a comprehensive phenotypical data collection of schizophrenic patients. BMC Psychiatry 2010; 10: 91

18 Wandinger KP, Saschenbrecker S, Stoecker W, Dalmau J. Anti-NMDA-receptor encephalitis: a severe, multistage, treatable disorder presenting with psychosis. J Neuroimmunol 2011; 231: 86-91.

19 Toyka KV, Brachman DB, Pestronk A, Kao I. Myasthenia gravis: passive transfer from man to mouse. Science 1975; 190: 397-399.

20 Piedrahita JA, Zhang SH, Hagaman JR, Oliver PM, Maeda N. Generation of mice carrying a mutant apolipoprotein $\mathrm{E}$ gene inactivated by gene targeting in embryonic stem cells. Proc Natl Acad Sci USA 1992; 89: 4471-4475.

21 Radyushkin K, El-Kordi A, Boretius S, Castaneda S, Ronnenberg A, Reim K et al. Complexin2 null mutation requires a 'second hit' for induction of phenotypic changes relevant to schizophrenia. Genes Brain Behav 2010; 9: 592-602.

22 Purcell S, Neale B, Todd-Brown K, Thomas L, Ferreira MA, Bender D et al. PLINK: a tool set for whole-genome association and population-based linkage analyses. Am J Hum Genet 2007; 81: 559-575.

23 Fullerton SM, Shirman GA, Strittmatter WJ, Matthew WD. Impairment of the blood-nerve and blood-brain barriers in apolipoprotein e knockout mice. Exp Neurol 2001; 169: 13-22.

24 Saher G, Rudolphi F, Corthals K, Ruhwedel T, Schmidt KF, Lowel S et al. Therapy of Pelizaeus-Merzbacher disease in mice by feeding a cholesterol-enriched diet. Nat Med 2012; 18: 1130-1135.

25 Bell RD, Winkler EA, Singh I, Sagare AP, Deane R, Wu Z et al. Apolipoprotein E controls cerebrovascular integrity via cyclophilin A. Nature 2012; 485: 512-516.

26 Shlosberg D, Benifla M, Kaufer D, Friedman A. Blood-brain barrier breakdown as a therapeutic target in traumatic brain injury. Nat Rev Neurol 2010; 6: 393-403.

27 Baburamani AA, Ek CJ, Walker DW, Castillo-Melendez M. Vulnerability of the developing brain to hypoxic-ischemic damage: contribution of the cerebral vasculature to injury and repair? Front Physiol 2012; 3: 424.

28 Visscher PM, Brown MA, McCarthy MI, Yang J. Five years of GWAS discovery. Am J Hum Genet 2012; 90: 7-24.

29 Zheng S, Eacker SM, Hong SJ, Gronostajski RM, Dawson TM, Dawson VL. NMDAinduced neuronal survival is mediated through nuclear factor I-A in mice. J Clin Invest 2010; 120: 2446-2456.

30 Sfriso $\mathrm{P}$, Ghirardello A, Botsios C, Tonon M, Zen M, Bassi N et al. Infections and autoimmunity: the multifaceted relationship. J Leukoc Biol 2010; 87: 385-395.

31 Ascherio A, Munger KL, Lunemann JD. The initiation and prevention of multiple sclerosis. Nat Rev Neurol 2012; 8: 602-612.

32 Dale RC, Irani SR, Brilot F, Pillai S, Webster R, Gill D et al. N-methyl-D-aspartate receptor antibodies in pediatric dyskinetic encephalitis lethargica. Ann Neurol 2009; 66: 704-709.

33 Tan A, Shuey N, Bladin C. A modern perspective on the differential diagnosis between encephalitis lethargica or anti-NMDA-receptor encephalitis. J Clin Neurosci 2010; 17: 1204-1206.

34 Reiber H, Peter JB. Cerebrospinal fluid analysis: disease-related data patterns and evaluation programs. J Neurol Sci 2001; 184: 101-122.

35 Ek CJ, Dziegielewska KM, Habgood MD, Saunders NR. Barriers in the developing brain and Neurotoxicology. Neurotoxicology 2012; 33: 586-604.

36 Grzeda E, Wisniewska RJ, Wisniewski K. Effect of an NMDA receptor agonist on T-maze and passive avoidance test in 12-week streptozotocin-induced diabetic rats. Pharmacol Rep 2007; 59: 656-663.

37 Sharp FR, Hendren RL. Psychosis: atypical limbic epilepsy versus limbic hyperexcitability with onset at puberty? Epilepsy Behav 2007; 10: 515-520.

38 Olney JW, Farber NB. Glutamate receptor dysfunction and schizophrenia. Arch Gen Psychiatry 1995; 52: 998-1007. 
39 Manto M, Dalmau J, Didelot A, Rogemond V, Honnorat J. Afferent facilitation of corticomotor responses is increased by $\mathrm{lgGs}$ of patients with NMDA-receptor antibodies. J Neurol 2011; 258: 27-33.

40 Bechter K, Reiber H, Herzog S, Fuchs D, Tumani H, Maxeiner HG. Cerebrospinal fluid analysis in affective and schizophrenic spectrum disorders: identification of subgroups with immune responses and blood-CSF barrier dysfunction. J Psychiatr Res 2010; 44: 321-330.

41 Doria A, Canova M, Tonon M, Zen M, Rampudda E, Bassi N et al. Infections as triggers and complications of systemic lupus erythematosus. Autoimmun Rev 2008; 8: 24-28.
42 Randone SB, Guiducci S, Cerinic MM. Systemic sclerosis and infections. Autoimmun Rev 2008; 8: 36-40.

43 Blanpied TA, Clarke RJ, Johnson JW. Amantadine inhibits NMDA receptors by accelerating channel closure during channel block. J Neurosci 2005; 25: 3312-3322.

44 Larrauri A, de Mateo S. Characterisation of swabbing for virological analysis in the Spanish Influenza Sentinel Surveillance System during four influenza seasons in the period 2002-2006. Euro Surveill 2007; 12: E5-E6.

45 Avitsur R, Mays JW, Sheridan JF. Sex differences in the response to influenza virus infection: modulation by stress. Horm Behav 2011; 59: 257-264.

Supplementary Information accompanies the paper on the Molecular Psychiatry website (http://www.nature.com/mp) 


\title{
Supplementary Appendix
}

This appendix has been provided by the authors to give readers additional information on their work.

\section{Neuropsychiatric disease relevance of circulating anti-NMDA receptor antibodies depends on blood brain barrier integrity}

\author{
Christian Hammer, Ph.D ${ }^{1}$, Beata Stepniak, M.Sc. ${ }^{1}$, Anja Schneider, M.D. ${ }^{2,3,4}$, Sergi Papiol, \\ Ph.D. ${ }^{1,3}$, Martesa Tantra, M.Sc. ${ }^{1,3}$, Martin Begemann, M.D. ${ }^{1}$, Anna-Leena Sirén, M.D., Ph.D. ${ }^{5}$, \\ Luis A. Pardo, M.D., Ph.D. ${ }^{6}$, Swetlana Sperling ${ }^{1}$, Suhaidah Mohd Jofrry, M.Sc. ${ }^{1}$, Artem Gurvich, \\ M.D. ${ }^{1}$, Niels Jensen, Ph.D. ${ }^{1}$, Katrin Ostmeier ${ }^{1}$, Fred Lühder, Ph.D. ${ }^{7}$, Christian Probst, Ph.D. ${ }^{8}$, \\ Henrik Martens, Ph.D. ${ }^{9}$, Meyke Gillis, M.D. ${ }^{10}$, Gesine Saher, Ph.D. ${ }^{11}$, Francesca Assogna, \\ Ph.D. ${ }^{12}$ Gianfranco Spalletta, M.D., Ph.D. ${ }^{12}$, Winfried Stöcker, M.D. ${ }^{8}$, Thomas F. Schulz, M.D. ${ }^{10}$, \\ Klaus-Armin Nave, Ph.D. ${ }^{3,11}$, and Hannelore Ehrenreich, M.D., D.V.M. ${ }^{1,3}$ \\ ${ }^{1}$ Division of Clinical Neuroscience, Max Planck Institute of Experimental Medicine, \\ Göttingen, Germany \\ ${ }^{2}$ Department of Psychiatry \& Psychotherapy, University Medicine Göttingen, \\ Göttingen, Germany \\ ${ }^{3}$ DFG Research Center Nanoscale Microscopy and Molecular Physiology of the Brain (CNMPB), \\ Göttingen, Germany \\ ${ }^{4}$ German Center for Neurodegenerative Diseases (DZNE), Göttingen, Germany \\ ${ }^{5}$ Department of Neurosurgery, University Clinic of Würzburg, Würzburg, Germany \\ ${ }^{6}$ Department of Molecular Biology of Neuronal Signals, Max Planck Institute of Experimental \\ Medicine, Göttingen, Germany \\ ${ }^{7}$ Department of Neuroimmunology, Institute for Multiple Sclerosis Research and Hertie \\ Foundation, University Medicine Göttingen, Göttingen, Germany \\ ${ }^{8}$ Institute for Experimental Immunology, affiliated to Euroimmun, Lübeck, Germany \\ ${ }^{9}$ Synaptic Systems GmbH, Göttingen, Germany \\ ${ }^{10}$ Institute of Virology, Hannover Medical School, Hannover, Germany \\ ${ }^{11}$ Department of Neurogenetics, Max Planck Institute of Experimental Medicine, \\ Göttingen, Germany \\ ${ }^{12}$ Department of Clinical and Behavioral Neurology, IRCCS Santa Lucia Foundation, Rome, Italy
}

Correspondence:

Hannelore Ehrenreich, MD, DVM

Division of Clinical Neuroscience

Max Planck Institute of Experimental Medicine

Göttingen, Germany

Phone +49-551-3899615

Fax +49-551-3899670

Email: ehrenreich@em.mpg.de 


\section{TABLE OF CONTENTS}

Table S1. Characteristics of sera used for in vivo and in vitro experiments 3

Table S2. Mean age of study cohorts by lg isotype $\quad 4$

$\begin{array}{lll}\text { Figure S1. } & \text { Age dependence of seropositivity } & 4\end{array}$

Table S3. Gender distribution of seropositive subjects by lg isotype 5

Table S4. Distribution of anti-NR1 AB titers 5

$\begin{array}{lll}\text { Figure S2. } & \text { Anti-NR1 AB titers: } 100 \% \text { stacked chart }\end{array}$

$\begin{array}{lll}\text { Figure S3. } & \text { Mouse AB injection experiments presented separately for isotypes } & 7\end{array}$

$\begin{array}{ll}\text { SNP array specifications and genotyping quality control } & 8\end{array}$

$\begin{array}{lll}\text { Table S5. } & \text { Marker selection criteria } & 8\end{array}$

Table S6. Patient and healthy control groups selected for genotyping 8

$\begin{array}{lll}\text { Table S7. Axiom } \AA \text { array quality control } & 9\end{array}$

$\begin{array}{ll}\text { Genome-wide association study (GWAS) } & 9\end{array}$

$\begin{array}{lll}\text { Figure S4. } & \text { Principal component analysis } & 9\end{array}$

$\begin{array}{lll}\text { Figure S5. } & \text { Q-Q plot } & 10\end{array}$

$\begin{array}{lll}\text { Figure S6. Manhattan plot } & 11\end{array}$

$\begin{array}{lll}\text { Figure S7. } & \text { SNP rs524991 regional association plot } & 11\end{array}$

Table S8. $\quad$ SNP rs524991 association statistics by study group $\quad 12$

$\begin{array}{lll}\text { Table S9. } & \text { HLA type association with seropositivity } & 13\end{array}$

Table S10. Influenza association with anti-NR1 seropositivity, gender-specific 14 
Table S1. Characteristics of sera used for in vivo and in vitro experiments

List of sera of patients and healthy controls used for determination of AB functionality in vitro (receptor endocytosis assay) and in vivo (mouse intravenous injection experiments). Total concentrations of the respective lg subclass in the ammonium sulfate precipitated serum fractions are consistent with published serum levels of immunoglobulins in the general adult population (Gonzalez-Quintela A, et al. Serum levels of immunoglobulins (IgG, IgA, IgM) in a general adult population and their relationship with alcohol consumption, smoking and common metabolic abnormalities. Clin Exp Immunol 2008;151:42-50).

\begin{tabular}{|c|c|c|c|c|c|c|c|c|c|c|c|}
\hline \multirow{2}{*}{ Ig isotype } & \multirow{2}{*}{ ID (code) } & \multirow{2}{*}{$\begin{array}{c}\text { Age } \\
\text { (years) }\end{array}$} & \multirow{2}{*}{ Gender } & \multirow{2}{*}{ Diagnosis } & \multirow{2}{*}{$\begin{array}{c}\text { Serum } \\
\text { titer }\end{array}$} & \multirow{2}{*}{$\begin{array}{c}\text { Titer } \\
\text { in Ig extract }\end{array}$} & \multicolumn{3}{|c|}{ Total concentration in fraction $(\mathrm{g} / \mathrm{l})$} & \multicolumn{2}{|c|}{ Usage of purified serum } \\
\hline & & & & & & & $\lg G$ & $\lg A$ & $\lg M$ & Endocytosis & Injection \\
\hline \multirow[t]{6}{*}{$\lg G$} & 706594 & 49 & $m$ & healthy control & 32 & 10 & 16.84 & 4.35 & 1.44 & $\sqrt{ }$ & \\
\hline & BPD00045 & 45 & $f$ & bipolar affective disorder & 100 & 32 & 4.25 & NA & NA & $\sqrt{ }$ & \\
\hline & SCZ00979 & 27 & $\mathrm{~m}$ & schizoaffective disorder & 320 & 100 & 11.50 & NA & NA & $\sqrt{ }$ & \\
\hline & SCZ01025 & 40 & $f$ & schizoaffective disorder & 100 & 100 & 14.37 & NA & NA & $\sqrt{ }$ & \\
\hline & UPD00066 & 32 & $f$ & major depressive disorder & 320 & 100 & 10.41 & NA & NA & $\sqrt{ }$ & \\
\hline & UPD00071 & 43 & $\mathrm{~m}$ & major depressive disorder & 1000 & 1000 & 20.13 & 1.92 & 1.26 & $\sqrt{ }$ & $\sqrt{ }$ \\
\hline \multirow[t]{5}{*}{$\lg A$} & 706026 & 39 & $\mathrm{~m}$ & healthy control & 3200 & 3200 & 13.78 & 1.57 & 2.09 & $\sqrt{ }$ & \\
\hline & 706472 & 37 & $\mathrm{~m}$ & healthy control & 1000 & 1000 & 18.13 & 3.42 & 2.44 & $\sqrt{ }$ & \\
\hline & SCZ00547 & 31 & $\mathrm{~m}$ & schizophrenia & 1000 & 1000 & 14.98 & 2.98 & 2.02 & $\sqrt{ }$ & \\
\hline & SCZ00857 & 43 & $f$ & schizophrenia & 1000 & 1000 & 20.81 & 4.86 & 1.15 & $\sqrt{ }$ & $\sqrt{ }$ \\
\hline & UPD00035 & 46 & $\mathrm{~m}$ & major depressive disorder & 320 & 320 & 13.78 & 4.86 & 1.50 & $\sqrt{ }$ & \\
\hline \multirow[t]{4}{*}{$\lg M$} & 707334 & 47 & $\mathrm{~m}$ & healthy control & 3200 & 1000 & 14.98 & 3.09 & 1.44 & $\sqrt{ }$ & \\
\hline & 708436 & 49 & $\mathrm{~m}$ & healthy control & 3200 & 320 & 21.51 & 2.88 & 1.32 & $\sqrt{ }$ & \\
\hline & SCZ00996 & 46 & $\mathrm{~m}$ & schizoaffective disorder & 1000 & 1000 & 32.20 & 4.86 & 1.56 & $\sqrt{ }$ & \\
\hline & ScZ00926 & 35 & $\mathrm{~m}$ & schizoaffective disorder & 1000 & 100 & 25.12 & 2.38 & 0.66 & $\sqrt{ }$ & $\sqrt{ }$ \\
\hline \multirow[t]{7}{*}{ Negative } & 707340 & 37 & $f$ & healthy control & NA & NA & 9.89 & 0.81 & 1.26 & $\sqrt{ }$ & \\
\hline & 708438 & 48 & $f$ & healthy control & NA & NA & 11.50 & 1.41 & 0.89 & $\sqrt{ }$ & \\
\hline & BPD00028 & 41 & $\mathrm{~m}$ & bipolar affective disorder & NA & NA & 16.21 & 5.52 & 1.21 & $\sqrt{ }$ & $\sqrt{ }$ (control for IgA) \\
\hline & SCZ00071 & 41 & $\mathrm{~m}$ & schizophrenia & NA & NA & 25.12 & 1.74 & 1.62 & $\sqrt{ }$ & $\sqrt{ }$ (control for Ig $\mathrm{G})$ \\
\hline & SCZ00097 & 46 & $f$ & schizophrenia & NA & NA & 13.20 & 1.17 & 1.69 & $\sqrt{ }$ & \\
\hline & SCZ00397 & 37 & $\mathrm{~m}$ & schizophrenia & NA & NA & 16.84 & 3.20 & 1.56 & $\sqrt{ }$ & \\
\hline & ScZ00437 & 43 & $\mathrm{~m}$ & schizophrenia & NA & NA & 17.48 & 1.10 & 1.38 & $\sqrt{ }$ & $\sqrt{ }$ (control for IgM) \\
\hline
\end{tabular}

m, male; f, female; NA, not available. 


\section{Table S2. Mean age of study cohorts by Ig isotype}

\begin{tabular}{lllllllll}
\hline & \multicolumn{5}{c}{ Mean age in years (SD) } \\
\cline { 2 - 7 } Study group & \multicolumn{5}{c}{ Seropositive } & \multirow{2}{*}{ Seronegative } \\
\cline { 2 - 8 } & all & IgG & IgA & IgM & \\
\hline GRAS patients & $41.1(12.0)$ & $34.0(11.7)$ & 42.0 & $(9.9)$ & $43.4(13.7)$ & $39.2(12.6)^{*}$ \\
Affective disorder patients & $47.4(11.9)^{*}$ & 45.6 & $(9.8)$ & 50.6 & $(9.8)^{*}$ & $41.9(11.0)$ & $50.2(16.1)^{*}$ \\
GRAS controls & $40.9(12.2)$ & $43.8(12.6)$ & $39.6(13.0)$ & $42.2(13.9)$ & $37.0(13.3)$ \\
\hline Parkinson patients & $69.1(8.3)$ & $52.00(N A)$ & 68.8 & $(8.4)$ & 68.1 & $(8.4)$ & $65.6(10.3)$ \\
Parkinson controls & $67.5(12.6)$ & NA & 70.3 & $(6.5)$ & $64.7(18.1)$ & $62.8(11.6)$ \\
\hline
\end{tabular}

* denotes significant difference $(p \leq 0.05)$ of patient group versus respective controls. $p$ values were calculated using 2-tailed independent samples t-tests, equal variances not assumed in case of significant Levene's test. SD, standard deviation; NA, not applicable.

Figure S1. Age dependence of seropositivity

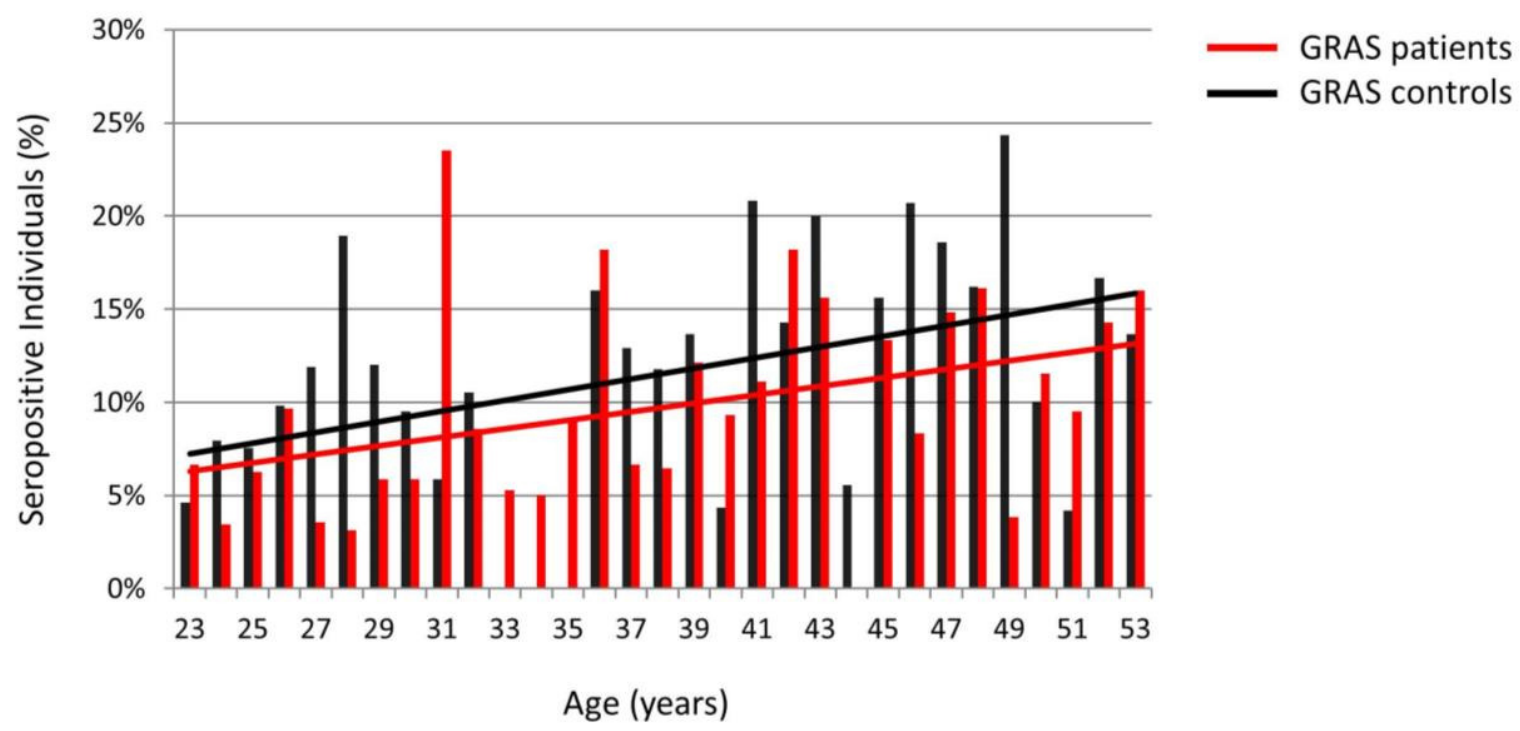

Dotted lines connect single data points (seroprevalence per year of age), solid lines represent linear trends. Only GRAS patients and GRAS controls were included due to the significantly smaller size of the other cohorts. Patients younger than 23 and older than 53 were not included due to low numbers per year of age. 
Table S3. Gender distribution of seropositive subjects by Ig isotype

\begin{tabular}{lrrr}
\hline \multirow{2}{*}{$\lg$ Isotype } & \multicolumn{2}{c}{ \% Seropositive } & \multirow{2}{*}{ (Pearson Chi $^{2}$ ) } \\
\cline { 2 - 3 } & Male (N=1769) & Female (N=1048) & $\mathbf{0 . 0 1 7}$ \\
\hline all & $11.53 \%$ & $8.68 \%$ & 0.260 \\
$\operatorname{lgG}$ & $0.51 \%$ & $0.86 \%$ & 0.052 \\
$\lg \mathrm{A}$ & $6.56 \%$ & $4.77 \%$ & 0.067 \\
\hline $\operatorname{lgM}$ & $6.33 \%$ & $4.68 \%$ & \\
\hline
\end{tabular}

Bolded values, $p<0.05$

Table S4. Distribution of anti-NR1 AB titers

\begin{tabular}{|c|c|c|c|c|c|c|c|c|c|}
\hline \multirow{3}{*}{$\frac{\text { Study cohort }}{\text { GRAS patients }}$} & \multirow{3}{*}{$\frac{\text { Isotype }}{\lg G}$} & \multicolumn{8}{|c|}{ Anti-NR1 AB titer (\% of seropositive subjects) } \\
\hline & & \multirow{2}{*}{$\begin{array}{l}1: 10 \\
2(28.6)\end{array}$} & \multirow{2}{*}{$\begin{array}{l}1: 32 \\
2(28.6)\end{array}$} & \multirow{2}{*}{$\frac{1: 100}{1(14.3)}$} & \multirow{2}{*}{\begin{tabular}{|l}
$1: 320$ \\
$2(28.6)$
\end{tabular}} & $1: 1000$ & $1: 3200$ & $1: 10000$ & \multirow{2}{*}{$\frac{\text { all }}{7(100.0)}$} \\
\hline & & & & & & $0 \quad(0.0)$ & $0 \quad(0.0)$ & $0 \quad(0.0)$ & \\
\hline & $\lg A$ & $13(23.3)$ & $21(37.5)$ & $10(17.9)$ & $5(8.9)$ & $6(10.7)$ & $1(1.8)$ & $0 \quad(0.0)$ & $56(100.0)$ \\
\hline & $\lg M$ & $9(19.6)$ & $10(21.7)$ & $16(34.8)$ & $5(10.9)$ & $5(10.9)$ & $0 \quad(0.0)$ & $1(2.2)$ & $46(100.0)$ \\
\hline \multirow[t]{3}{*}{ Affective disorder patients } & $\lg G$ & $0 \quad(0.0)$ & $2(40.0)$ & $1(20.0)$ & $1(20.0)$ & $1(20.0)$ & $0 \quad(0.0)$ & $0 \quad(0.0)$ & $5(100.0)$ \\
\hline & $\lg A$ & $3(20.0)$ & $6(40.0)$ & $4(26.7)$ & $2(13.3)$ & $0 \quad(0.0)$ & $0 \quad(0.0)$ & $0 \quad(0.0)$ & $15(100.0)$ \\
\hline & $\lg M$ & $0 \quad(0.0)$ & $1(14.3)$ & $4(57.1)$ & $1(14.3)$ & $1(14.3)$ & $0 \quad(0.0)$ & $\begin{array}{ll}0 & (0.0)\end{array}$ & $7(100.0)$ \\
\hline \multirow[t]{3}{*}{ GRAS controls } & $\lg G$ & $0 \quad(0.0)$ & $1(20.0)$ & $2(40.0)$ & $2(40.0)$ & $0 \quad(0.0)$ & $0 \quad(0.0)$ & $0 \quad(0.0)$ & $5(100.0)$ \\
\hline & $\lg A$ & $16(21.3)$ & $21(28.0)$ & $29(38.7)$ & $6(8.0)$ & 1 (1.3) & $2(2.7)$ & $0 \quad(0.0)$ & $75(100.0)$ \\
\hline & $\lg M$ & $9(11.3)$ & $16(20.0)$ & $40(50.0)$ & $7 \quad(8.8)$ & $6 \quad(7.5)$ & $2(2.5)$ & $0 \quad(0.0)$ & $80(100.0)$ \\
\hline \multirow[t]{3}{*}{ Parkinson patients } & $\lg G$ & $0 \quad(0.0)$ & $0 \quad(0.0)$ & $0 \quad(0.0)$ & $1(100.0)$ & $0 \quad(0.0)$ & $0 \quad(0.0)$ & $0 \quad(0.0)$ & $1(100.0)$ \\
\hline & $\lg A$ & $4(23.5)$ & $3(17.6)$ & $6(35.3)$ & $2(11.8)$ & $2(11.8)$ & $0 \quad(0.0)$ & $0 \quad(0.0)$ & $17(100.0)$ \\
\hline & $\lg M$ & $4(16.0)$ & $9(36.0)$ & $10(40.0)$ & $1(4.0)$ & $1(4.0)$ & $0 \quad(0.0)$ & $0 \quad(0.0)$ & $25(100.0)$ \\
\hline \multirow[t]{3}{*}{ Parkinson controls } & $\lg G$ & $0 \quad(0.0)$ & $0 \quad(0.0)$ & $0 \quad(0.0)$ & $0 \quad(0.0)$ & $0 \quad(0.0)$ & $0 \quad(0.0)$ & $0 \quad(0.0)$ & $0 \quad(0.0)$ \\
\hline & $\lg A$ & $0 \quad(0.0)$ & $2(66.7)$ & $1(33.3)$ & $0 \quad(0.0)$ & $0 \quad(0.0)$ & $0 \quad(0.0)$ & $0 \quad(0.0)$ & $3(100.0)$ \\
\hline & $\lg M$ & $0 \quad(0.0)$ & $2(66.7)$ & 1 (33.3) & $0 \quad(0.0)$ & $\begin{array}{ll}0 & (0.0)\end{array}$ & $\begin{array}{ll}0 & (0.0)\end{array}$ & $0 \quad(0.0)$ & $3(100.0)$ \\
\hline
\end{tabular}

Mann-Whitney U-rank test revealed no difference between groups regarding titers. 
Figure S2. Anti-NR1 AB titers: $100 \%$ stacked chart

\section{a. $\lg G$}

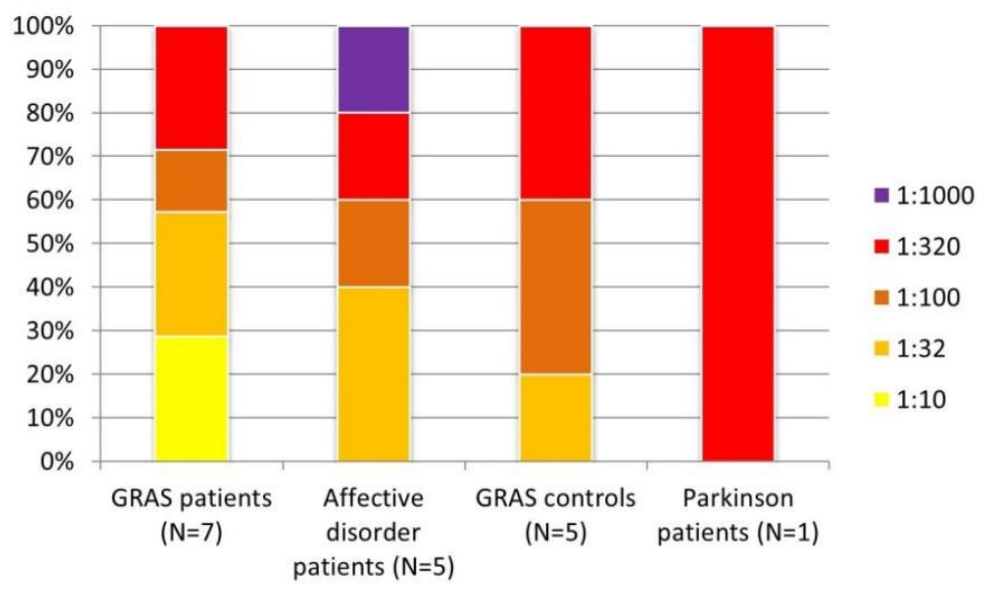

b. $\lg A$

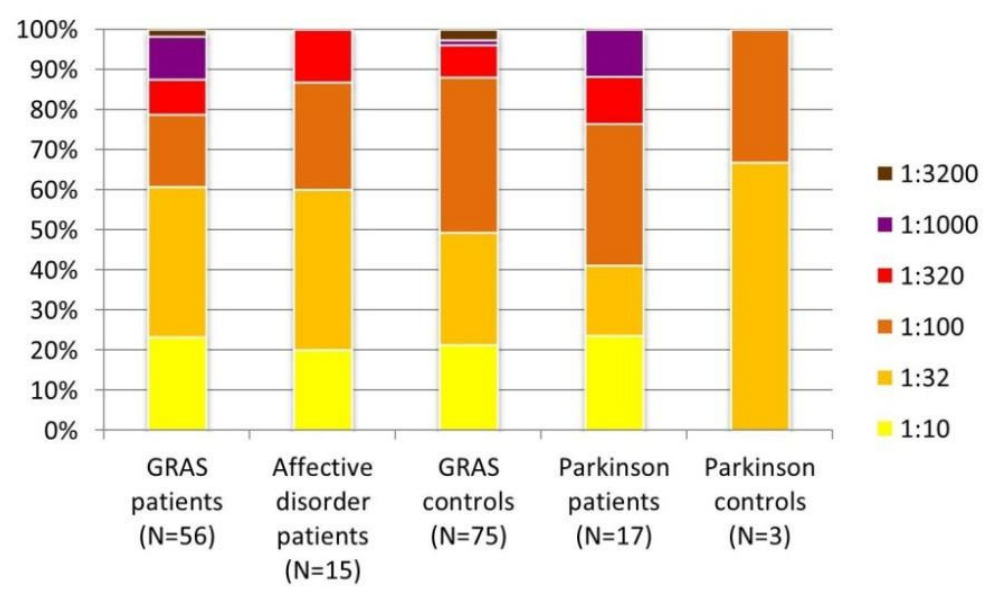

\section{c. $\lg M$}

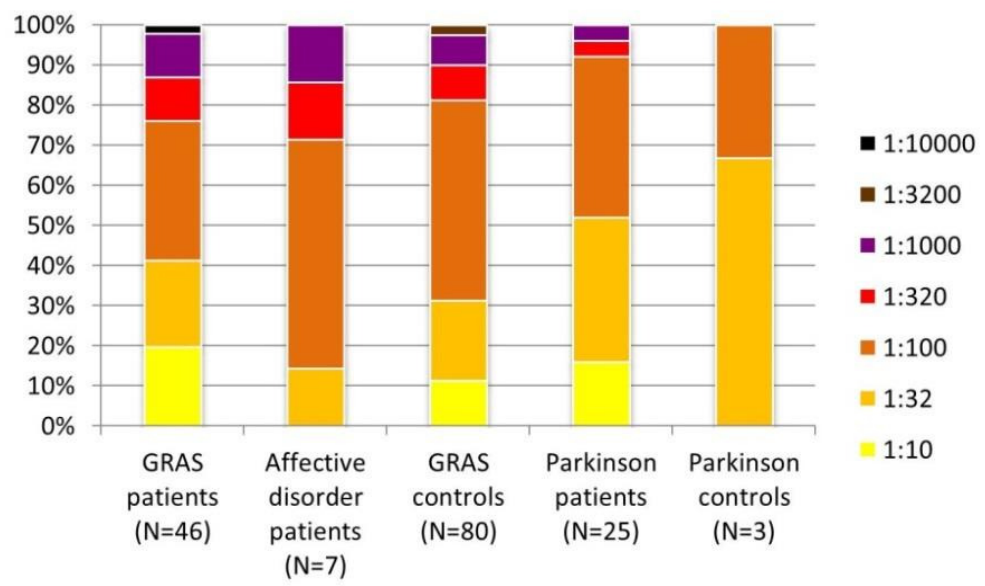


Figure S3. Mouse AB injection experiments presented separately for isotypes

(Note: The IgM set of experiments includes a non-injection group to exclude effects of injection on spontaneous open field activity)

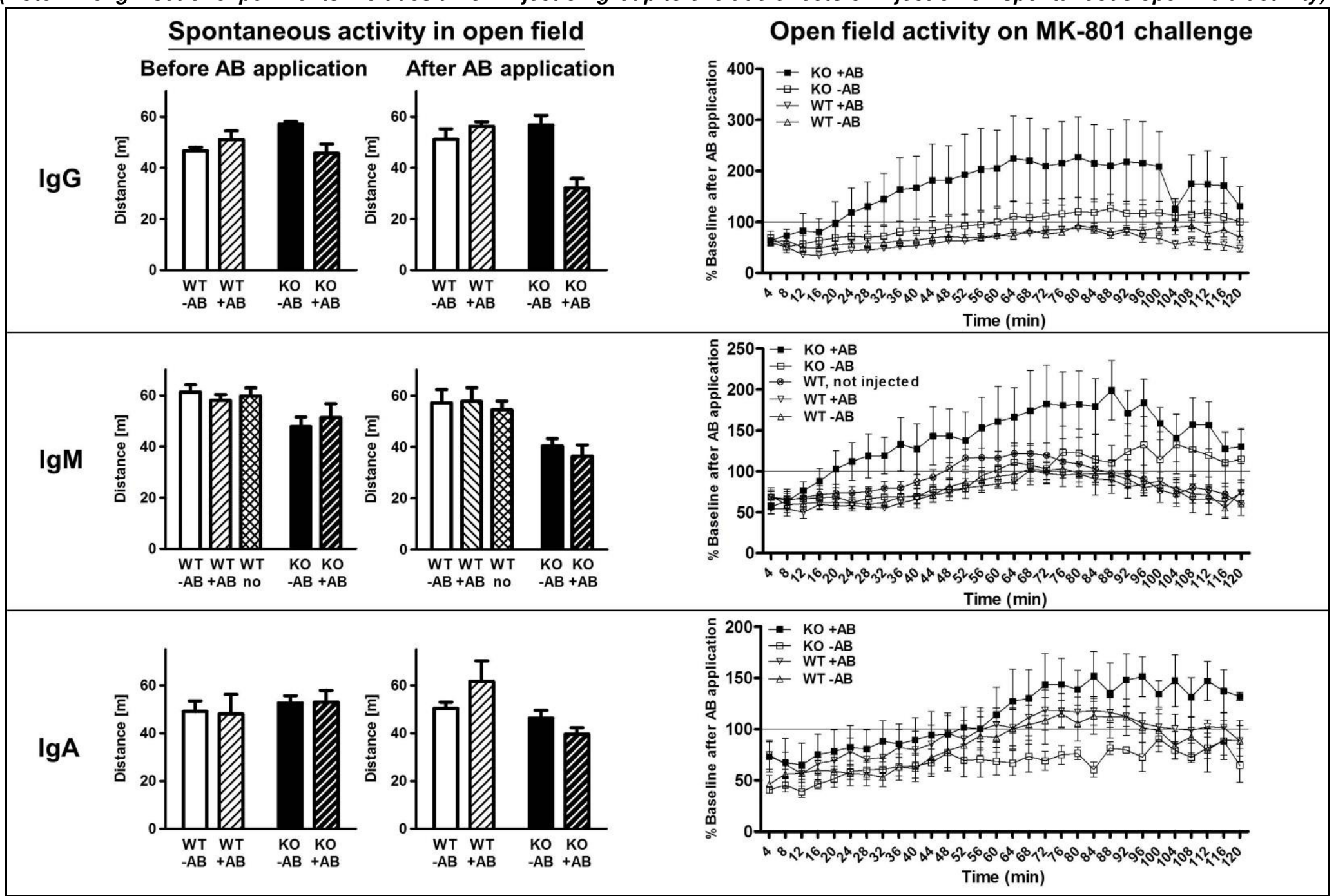

Left panels: Spontaneous activity in the open field - Total distance traveled before and after Ig injection. Right panels: Open field activity upon MK-801 challenge.

$\mathrm{m}$, meter; WT, wildtype; KO, ApoE ${ }^{-/}$knockout; -AB, NMDAR-AB seronegative; +AB, NMDAR-AB seropositive; no, non-injection control; N=4-6 per group. 


\section{SNP array specifications and genotyping quality control}

Genotyping was performed using a semi-custom Axiom® myDesign ${ }^{\mathrm{TM}}$ genotyping array (Affymetrix, Santa Clara, CA, USA), based on a CEU (Caucasian residents of European ancestry from Utah, USA) marker backbone including 518.722 SNPs, and a custom marker set including 102.537 SNPs. The array was designed using the Axiom $\AA$ Design center (www.affymetrix.com), applying diverse selection criteria (Table S5). Genotyping was performed by Affymetrix on a GeneTitan $\AA^{\circledR}$ platform.

\section{Table S5. Marker selection criteria}

\begin{tabular}{llr}
\hline Marker group & Threshold & \# of markers \\
\hline Axiom CEU backbone & MAF > 0.025, direct selection & 518,722 \\
Functional Variants (CDS, UTR, exonic) & MAF > 0.2, direct selection & 27,369 \\
Markers contained in RefSeq / UCSC genes (+/- 2kbp) & MAF > 0.2, r² > 0.8 & 31,190 \\
Markers in the MHC genomic region & best tag & 15,395 \\
Markers in CpG islands & MAF > 0.2, direct selection & 5,511 \\
Markers in known splice sites & MAF > 0.1, direct selection & 95 \\
Markers in microRNA regions & MAF > 0.2, direct selection & 56 \\
Manual selection of candidate SNPs & MAF > 0.2, best tag & 22,921 \\
Affymetrix SNPs for quality control & & 3,334 \\
TOTAL & & $\mathbf{6 2 4 , 5 9 3}$ \\
\hline
\end{tabular}

MAF, minor allele frequency; CDS, coding sequence; UTR, untranslated region; RefSeq, NCBI reference sequence; UCSC, University of California Santa Cruz Genome Browser; kbp, kilo base pairs; MHC, major histocompatibility complex.

In total, 3128 individuals were subjected to genotyping (Table S6).

Table S6. Patient and healthy control groups selected for genotyping

\begin{tabular}{lr}
\hline Study group & $\mathbf{N}(\%)$ \\
\hline GRAS patients & $1177(37.6)$ \\
Affective disorder patients & $173(5.5)$ \\
GRAS controls & $1274(40.7)$ \\
Parkinson patients & $276(8.8)$ \\
Parkinson controls & $182(5.8)$ \\
Autism patients & $29(0.9)$ \\
Other conditions & $17(0.5)$ \\
TOTAL & $\mathbf{3 1 2 8}(\mathbf{1 0 0})$ \\
\hline
\end{tabular}

Quality control (QC) was performed on sample and SNP level, according to the manufacturer's recommendations (Table S7). A total of 3.086 individuals and 612.121 SNPs passed all quality controls to be included in genetic analyses. 
Table S7. Axiom $\circledast$ array quality control

\begin{tabular}{lllr}
\hline & Filter criteria & $\begin{array}{l}\text { Threshold for } \\
\text { inclusion }\end{array}$ & \multicolumn{1}{c}{$\mathbf{N}$} \\
\hline Sample & & & Samples passing QC step \\
level & & & $(\%)$ \\
& Pre QC & $\geq 0.82$ & $3,128(100)$ \\
& Dish QC & $\geq 0.97$ & $3,124(99.8)$ \\
& Cluster call rate & & $3,086(98.7)$ \\
\hline SNP level & & $\geq 0.97$ & $624,593(100)$ \\
& Pre QC & $\geq 3.6$ & \\
& SNP call rate & $\geq-0.1$ & \\
& Fisher's Linear Discriminant (FLD) & SNPs passing QC step (\%) \\
& Heterozygous Cluster Strength Offset & & \\
& (HetSO) & $\geq 0.3$ & $612,121(98.0)$ \\
\hline
\end{tabular}

\section{Genome-wide association study (GWAS)}

GRAS and affective disorder patients, as well as the healthy GRAS controls, were collected in Germany, whereas the Parkinson samples and controls were collected in Italy. An analysis of principal components (PCA) was performed, showing a clear clustering of samples by descent (Figure S4). Therefore, to reduce population stratification bias, GWAS was performed including only GRAS and affective disorder patients, as well as GRAS controls.

Figure S4. Principal component analysis

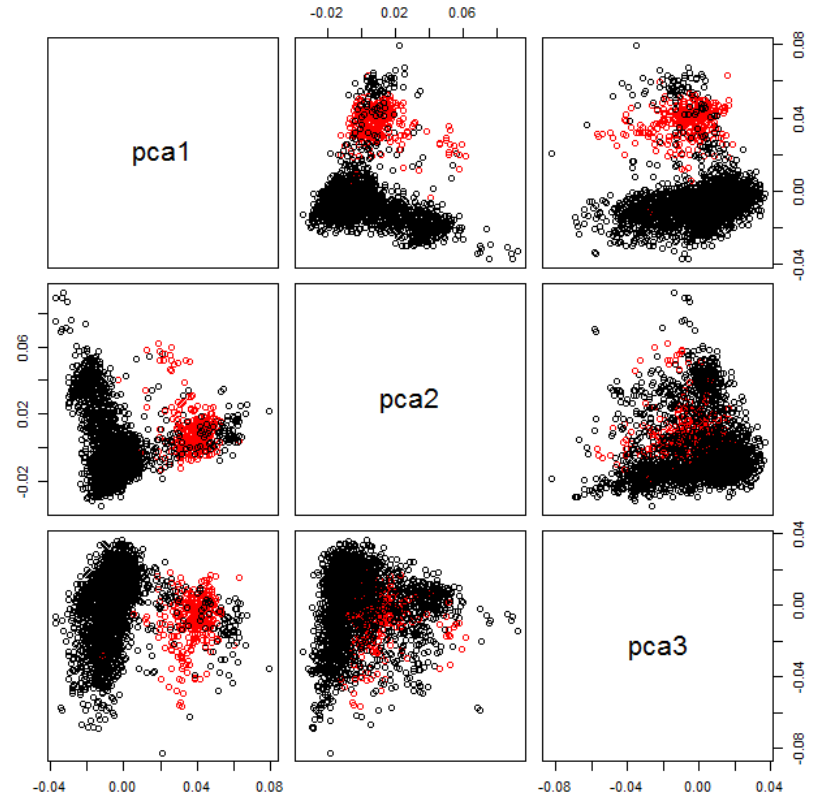

Graphical presentation including all individuals showing the first 3 principal components. Samples collected in Germany are depicted in black, samples collected in Italy in red. 
For the GWAS, PLINK v1.07 was used to calculate association statistics, applying a basic allelic model, and using the following filter criteria for SNP and sample exclusion:

- Markers located on sex chromosomes

- Hardy-Weinberg $p<5 \times 10^{-7}$ in controls

- Minor allele frequency $<0.01$

- missingness per marker $>0.05$

- missingness per individual $>0.02$

Overall, a total of $\mathbf{5 8 0 . 2 9 7}$ SNPs remained for analysis. The final dataset consisted of 245 cases (anti-NR1 positive) and 2152 controls (anti-NR1 negative).

A Q-Q plot was generated to visualize possible confounders (Figure S5). The genomic inflation factor was $\lambda=1.01$ (Devlin B, Roeder K. Genomic control for association studies. Biometrics 1999;55:997-1004). This low genomic inflation value justifies not including principal components as covariates after removal of the Italian Parkinson patient and control samples.

Figure S5. Q-Q plot

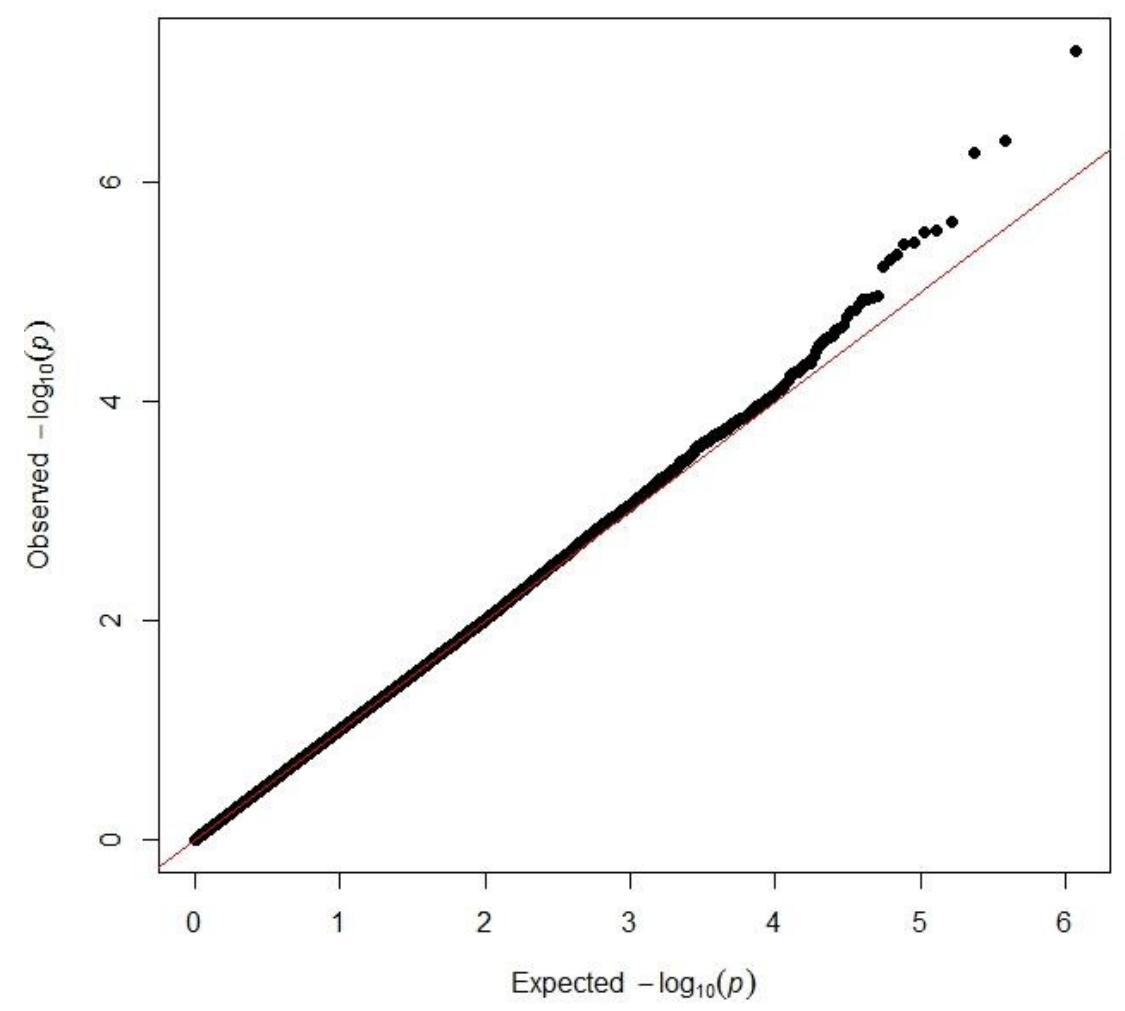


Figure S6. Manhattan plot

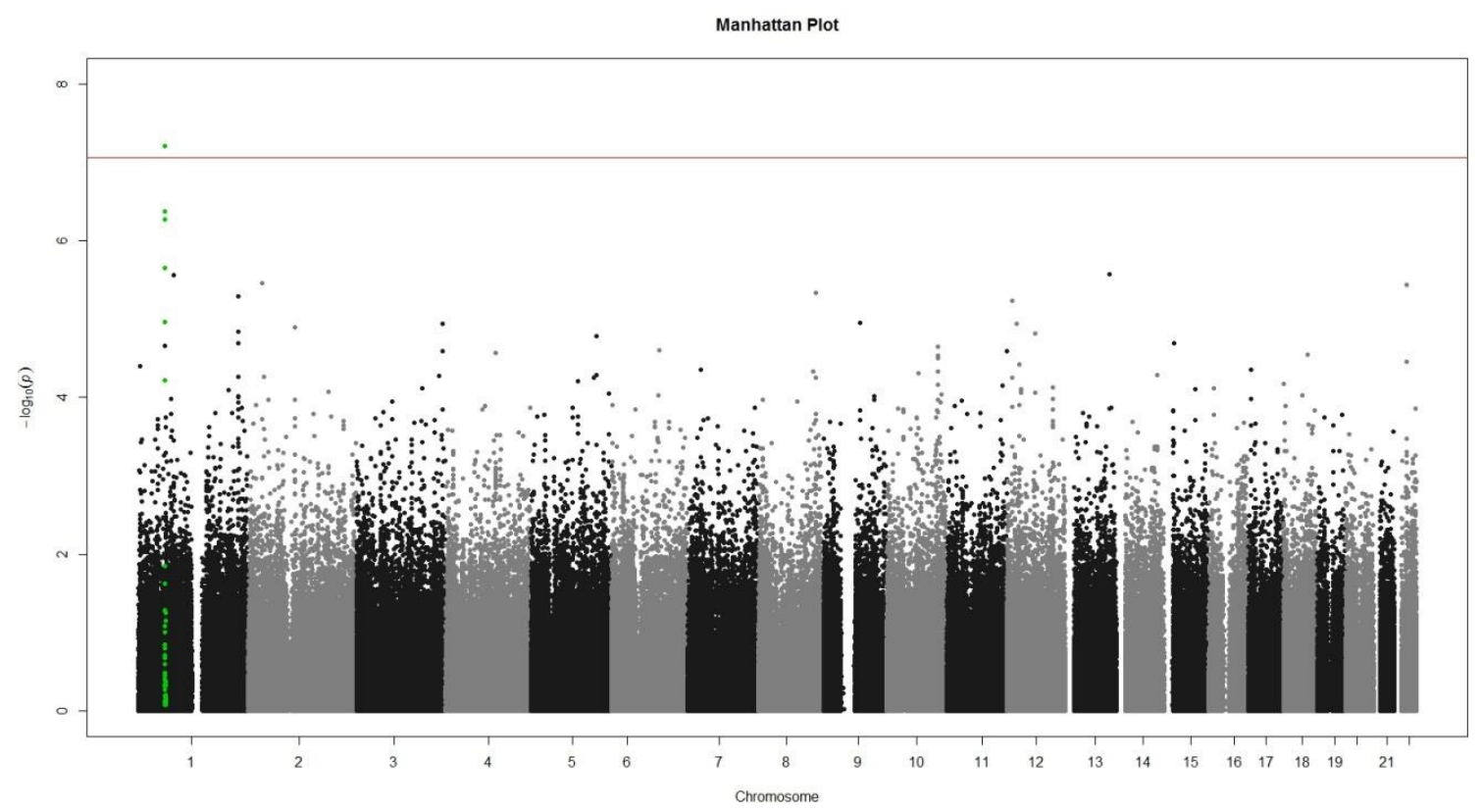

Manhattan plot of single SNP test statistics. The genomic position is shown on the $X$ axis, and the $-\log _{10}(p)$ on the $\mathrm{Y}$ axis. The horizontal line denotes the threshold for significance after Bonferroni correction for 580.297 tests ( $p=8.62 \mathrm{E}-08$ ). A marker on chromosome 1 (rs524991) showing genome-wide significance, as well as surrounding SNPs (+/- 50kbp) are depicted in green.

\section{Figure S7. SNP rs524991 regional association plot}

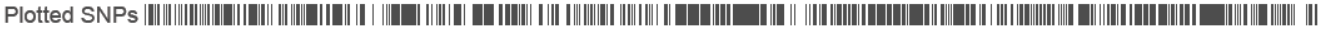

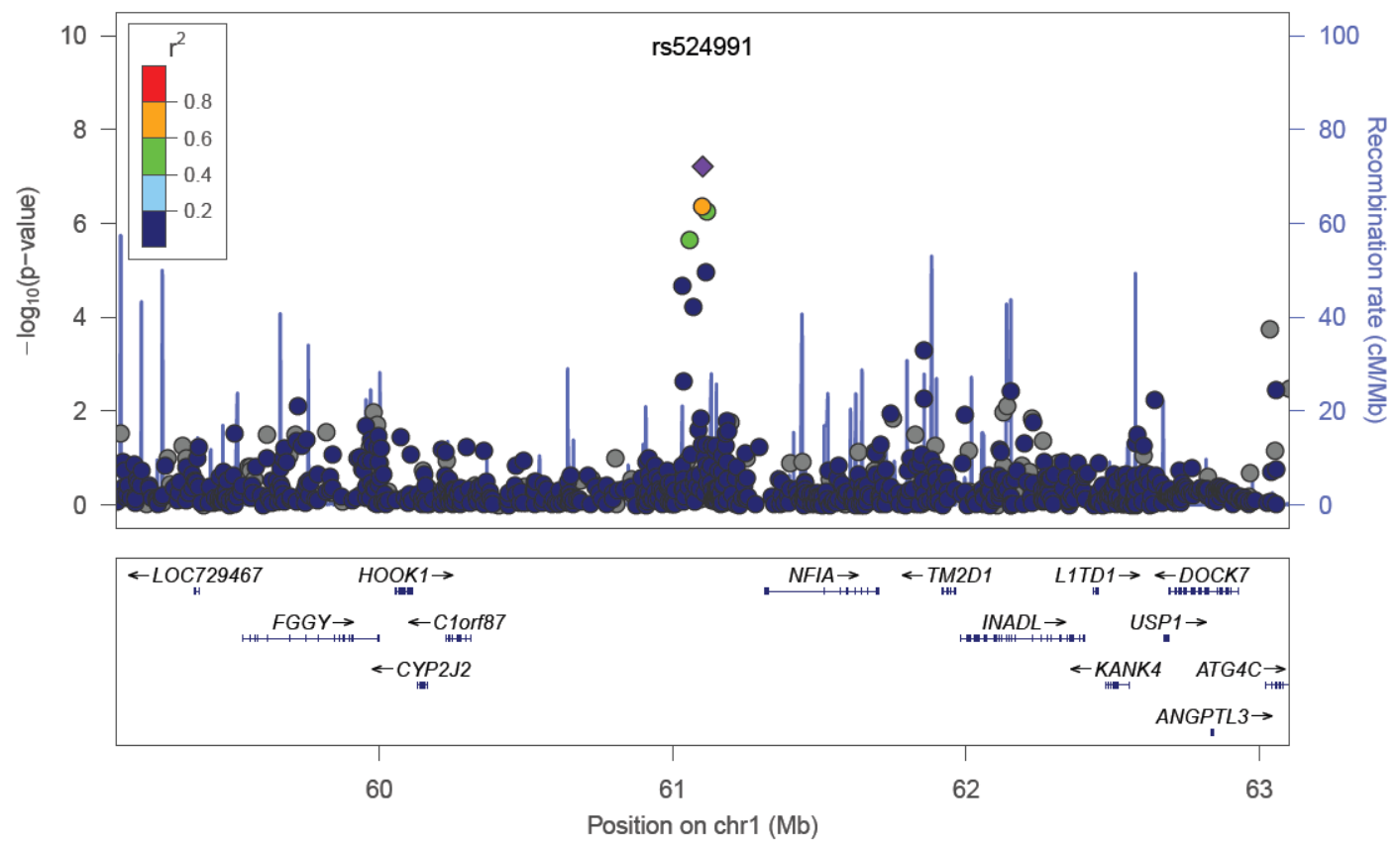

Regional association plot showing 2000kbp 5' and $3^{\prime}$ of rs524991 ( $p=8.62 \mathrm{E}-08$ ). The positions of neighboring genes are shown on the $\mathrm{X}$ axis, and the $-\log _{10}(\mathrm{p})$ on the $\mathrm{Y}$ axis. Color coding denotes LD information (see legend within the plot). 
Table S8. SNP rs524991 association statistics by study group

\begin{tabular}{|c|c|c|c|c|c|c|c|}
\hline Study group & $\begin{array}{l}\text { Anti- } \\
\text { NR1 }\end{array}$ & $\mathbf{N}$ & \multicolumn{3}{|c|}{ Genotypes (frequencies) } & $\begin{array}{r}p \text { (trend test) } \\
\text { d.f.=1 }\end{array}$ & $\begin{array}{r}p(\mathrm{OR},[95 \% \mathrm{CI}]) \\
\text { allelic, d.f. }=1\end{array}$ \\
\hline Both genders & & & GG (\%) & GA (\%) & $\mathrm{AA}(\%)$ & & \\
\hline GRAS patients & $\begin{array}{l}+ \\
-\end{array}$ & $\begin{array}{r}92 \\
976\end{array}$ & $\begin{array}{r}65(70.7) \\
867(88.8)\end{array}$ & $\begin{array}{r}27(29.3) \\
105(10.8)\end{array}$ & $\begin{array}{l}0(0.0) \\
4(0.4)\end{array}$ & 2.92E-06 & 3.23E-06 (2.80, [1.78 - 4.39]) \\
\hline $\begin{array}{l}\text { Affective disorder } \\
\text { patients }\end{array}$ & $\begin{array}{l}+ \\
-\end{array}$ & $\begin{array}{r}24 \\
119\end{array}$ & $\begin{array}{r}18(75.0) \\
104(87.4)\end{array}$ & $\begin{array}{r}6(25.0) \\
15(12.6)\end{array}$ & $\begin{array}{l}0(0.0) \\
0(0.0)\end{array}$ & 0.118 & $0.133(2.12,[0.78-5.79])$ \\
\hline GRAS controls & $\begin{array}{l}+ \\
-\end{array}$ & $\begin{array}{r}135 \\
1115\end{array}$ & $\begin{array}{l}111(82.2) \\
977(87.6)\end{array}$ & $\begin{array}{r}19(14.1) \\
135(12.1)\end{array}$ & $\begin{array}{l}5(3.7) \\
3(0.3)\end{array}$ & 0.007 & $0.006(1.78,[1.17-2.72]$ \\
\hline Parkinson patients & $\begin{array}{l}+ \\
-\end{array}$ & $\begin{array}{r}35 \\
220\end{array}$ & $\begin{array}{r}31(88.6) \\
187(85.0)\end{array}$ & $\begin{array}{r}4(11.4) \\
32(14.5)\end{array}$ & $\begin{array}{l}0(0.0) \\
1(4.6)\end{array}$ & 0.547 & $0.551 \quad(0.72,[0.25-2.11])$ \\
\hline Parkinson controls & $\begin{array}{l}+ \\
-\end{array}$ & $\begin{array}{r}6 \\
45\end{array}$ & $\begin{array}{r}4(66.7) \\
41(91.1)\end{array}$ & $\begin{array}{r}2(33.3) \\
4 \quad(8.9)\end{array}$ & $\begin{array}{l}0(0.0) \\
0(0.0)\end{array}$ & 0.081 & $0.091(4.30,[0.70-26.52])$ \\
\hline TOTAL & + & $\begin{array}{r}292 \\
2475 \\
\end{array}$ & $\begin{array}{r}229(78.4) \\
2176(87.9) \\
\end{array}$ & $\begin{array}{r}58(19.9) \\
291(11.8) \\
\end{array}$ & $\begin{array}{l}5(1.7) \\
8(0.3) \\
\end{array}$ & 7.64E-07 & 7.48E-07 (1.99, [1.51 - 2.63]) \\
\hline Males only & & & $\mathrm{GG}(\%)$ & $\mathrm{GA}(\%)$ & $\mathrm{AA}(\%)$ & & \\
\hline GRAS patients & $\begin{array}{l}+ \\
-\end{array}$ & $\begin{array}{r}67 \\
649\end{array}$ & $\begin{array}{r}51(76.1) \\
579(89.2)\end{array}$ & $\begin{array}{l}16(23.9) \\
67(10.3)\end{array}$ & $\begin{array}{l}0(0.0) \\
3(0.5)\end{array}$ & 0.004 & $0.004(2.28,[1.28-4.04])$ \\
\hline $\begin{array}{l}\text { Affective disorder } \\
\text { patients }\end{array}$ & $\begin{array}{l}+ \\
-\end{array}$ & $\begin{array}{l}11 \\
55\end{array}$ & $\begin{array}{r}9(81.8) \\
51(92.7)\end{array}$ & $\begin{array}{rr}2 & (18.2) \\
4 & (7.2)\end{array}$ & $\begin{array}{l}0(0.0) \\
0(0.0)\end{array}$ & 0.251 & $0.262(2.65,[0.45-15.45])$ \\
\hline GRAS controls & $\begin{array}{l}+ \\
-\end{array}$ & $\begin{array}{r}92 \\
646\end{array}$ & $\begin{array}{r}76(82.6) \\
562(87.0)\end{array}$ & $\begin{array}{l}13(14.1) \\
83(12.8)\end{array}$ & $\begin{array}{ll}3 & (3.3) \\
1 & (0.2)\end{array}$ & 0.064 & $0.063 \quad(1.64,[0.97-2.76])$ \\
\hline Parkinson patients & $\begin{array}{l}+ \\
-\end{array}$ & $\begin{array}{r}28 \\
140\end{array}$ & $\begin{array}{r}25(89.3) \\
118(84.3)\end{array}$ & $\begin{array}{r}3(10.7) \\
21(15.0)\end{array}$ & $\begin{array}{l}0(0.0) \\
1(0.7)\end{array}$ & 0.465 & $0.465 \quad(0.63,[0.18-2.18])$ \\
\hline Parkinson controls & $\begin{array}{l}+ \\
-\end{array}$ & $\begin{array}{r}2 \\
19\end{array}$ & $\begin{array}{r}1(50.0) \\
18(94.7)\end{array}$ & $\begin{array}{rr}1 & (50.0) \\
1 & (5.3)\end{array}$ & $\begin{array}{l}0(0.0) \\
0(0.0)\end{array}$ & 0.04 & $0.046(12.3,[0.61-250.5])$ \\
\hline TOTAL & $\begin{array}{l}+ \\
- \\
\end{array}$ & $\begin{array}{r}200 \\
1509 \\
\end{array}$ & $\begin{array}{r}162(81.0) \\
1328(88.0) \\
\end{array}$ & $\begin{array}{r}35(17.5) \\
176(11.7) \\
\end{array}$ & $\begin{array}{l}3(1.5) \\
5(3.3) \\
\end{array}$ & 0.002 & 2.04E-03 (1.74, [1.22 - 2.48]) \\
\hline Females only & & & GG (\%) & GA (\%) & $\mathrm{AA}(\%)$ & & \\
\hline GRAS patients & $\begin{array}{l}+ \\
-\end{array}$ & $\begin{array}{r}25 \\
327\end{array}$ & $\begin{array}{r}14(56.0) \\
288(88.1)\end{array}$ & $\begin{array}{l}11(44.0) \\
38(11.6)\end{array}$ & $\begin{array}{l}0(0.0) \\
1(0.3)\end{array}$ & 2.11E-05 & 2.97E-05 (4.33, [2.06 - 9.09]) \\
\hline $\begin{array}{l}\text { Affective disorder } \\
\text { patients }\end{array}$ & $\begin{array}{l}+ \\
-\end{array}$ & $\begin{array}{l}13 \\
63\end{array}$ & $\begin{array}{r}9(69.2) \\
52(82.5)\end{array}$ & $\begin{array}{r}4(30.8) \\
11(17.5)\end{array}$ & $\begin{array}{l}0(0.0) \\
0(0.0)\end{array}$ & 0.272 & $0.300(1.90,[0.55-6.52])$ \\
\hline GRAS controls & $\begin{array}{l}+ \\
-\end{array}$ & $\begin{array}{r}39 \\
421\end{array}$ & $\begin{array}{r}31(79.5) \\
371(88.1)\end{array}$ & $\begin{array}{r}6(15.4) \\
49(11.6)\end{array}$ & $\begin{array}{l}2(5.1) \\
1(0.2)\end{array}$ & 0.024 & $0.022(2.28,[1.11-4.69])$ \\
\hline Parkinson patients & $\begin{array}{l}+ \\
-\end{array}$ & $\begin{array}{r}7 \\
80\end{array}$ & $\begin{array}{r}6(85.7) \\
69(86.3)\end{array}$ & $\begin{array}{r}1(14.3) \\
11(13.7)\end{array}$ & $\begin{array}{l}0(0.0) \\
0(0.0)\end{array}$ & 0.969 & $0.970(1.04,[0.12-8.82])$ \\
\hline Parkinson controls & $\begin{array}{l}+ \\
-\end{array}$ & $\begin{array}{r}4 \\
26\end{array}$ & $\begin{array}{r}3(75.0) \\
23(88.5)\end{array}$ & $\begin{array}{l}1(25.0) \\
3(11.5)\end{array}$ & $\begin{array}{l}0(0.0) \\
0(0.0)\end{array}$ & 0.461 & $0.477(2.33,[0.21-25.66])$ \\
\hline TOTAL & $\begin{array}{l}+ \\
- \\
\end{array}$ & $\begin{array}{r}88 \\
917 \\
\end{array}$ & $\begin{array}{r}63(71.6) \\
803(87.6)\end{array}$ & $\begin{array}{r}23(26.1) \\
112(12.2) \\
\end{array}$ & $\begin{array}{l}2(2.3) \\
2(0.2)\end{array}$ & 7.42E-06 & 8.81E-06 (2.68, [1.71 - 4.21]) \\
\hline
\end{tabular}

Bolded values, $p<0.05$; d.f., degrees of freedom; OR, odds ratio; $\mathrm{Cl}$, confidence intervals; -, seronegative; +, seropositive. 
Table S9. HLA type association with seropositivity

\begin{tabular}{|c|c|c|c|c|c|c|c|}
\hline \multirow{2}{*}{ HLA allele } & \multicolumn{2}{|c|}{ Anti-NR1 seropositivity (\%) } & \multirow{2}{*}{$\begin{array}{c}p \\
\begin{array}{c}p \\
\text { (Pearson's } \\
\text { chi }^{2} \text { ) }\end{array}\end{array}$} & \multirow{2}{*}{ OR } & \multicolumn{2}{|c|}{$\mathrm{Cl} 95 \%$} & \multirow{2}{*}{$\begin{array}{l}\text { Imputation } \\
\text { quality (mean) }\end{array}$} \\
\hline & $-(\mathrm{N}=2483)$ & $+(\mathrm{N}=294)$ & & & low & high & \\
\hline \multicolumn{8}{|l|}{ HLA-A } \\
\hline 1 & $14.0 \%$ & $12.6 \%$ & 0.343 & 0.88 & 0.68 & 1.14 & 0.954 \\
\hline 2 & $29.7 \%$ & $28.7 \%$ & 0.615 & 0.95 & 0.79 & 1.15 & 0.937 \\
\hline 3 & $15.6 \%$ & $19.7 \%$ & 0.010 & 1.33 & 1.07 & 1.65 & 0.944 \\
\hline 11 & $5.7 \%$ & $4.8 \%$ & 0.370 & 0.83 & 0.56 & 1.24 & 0.943 \\
\hline 24 & $9.6 \%$ & $10.0 \%$ & 0.727 & 1.05 & 0.79 & 1.40 & 0.907 \\
\hline \multicolumn{8}{|l|}{ HLA-B } \\
\hline 7 & $13.3 \%$ & $13.1 \%$ & 0.874 & 0.98 & 0.76 & 1.26 & 0.833 \\
\hline 8 & $9.8 \%$ & $8.5 \%$ & 0.305 & 0.85 & 0.63 & 1.16 & 0.940 \\
\hline 15 & $8.4 \%$ & $8.5 \%$ & 0.917 & 1.02 & 0.75 & 1.38 & 0.616 \\
\hline 18 & $5.5 \%$ & $4.9 \%$ & 0.554 & 0.89 & 0.60 & 1.32 & 0.570 \\
\hline 35 & $10.6 \%$ & $12.4 \%$ & 0.168 & 1.20 & 0.93 & 1.56 & 0.476 \\
\hline 40 & $6.4 \%$ & $7.1 \%$ & 0.467 & 1.13 & 0.81 & 1.58 & 0.554 \\
\hline 44 & $11.9 \%$ & $9.9 \%$ & 0.150 & 0.81 & 0.61 & 1.08 & 0.869 \\
\hline 51 & $6.2 \%$ & $5.4 \%$ & 0.490 & 0.88 & 0.60 & 1.27 & 0.752 \\
\hline \multicolumn{8}{|l|}{$\mathrm{HLA}-\mathrm{C}$} \\
\hline 2 & $5.2 \%$ & $5.3 \%$ & 0.937 & 1.02 & 0.69 & 1.49 & 0.973 \\
\hline 3 & $13.2 \%$ & $13.1 \%$ & 0.927 & 0.99 & 0.77 & 1.27 & 0.945 \\
\hline 4 & $12.8 \%$ & $15.3 \%$ & 0.095 & 1.23 & 0.96 & 1.56 & 0.967 \\
\hline 5 & $7.1 \%$ & $7.0 \%$ & 0.932 & 0.99 & 0.70 & 1.38 & 0.967 \\
\hline 6 & $10.2 \%$ & $11.2 \%$ & 0.463 & 1.11 & 0.84 & 1.45 & 0.963 \\
\hline 7 & $31.0 \%$ & $30.4 \%$ & 0.793 & 0.98 & 0.81 & 1.17 & 0.957 \\
\hline 12 & $7.1 \%$ & $6.8 \%$ & 0.798 & 0.96 & 0.68 & 1.34 & 0.947 \\
\hline \multicolumn{8}{|l|}{ HLA-DPB1 } \\
\hline 1 & $5.2 \%$ & $4.8 \%$ & 0.624 & 0.91 & 0.61 & 1.35 & 0.885 \\
\hline 2 & $13.9 \%$ & $14.3 \%$ & 0.785 & 1.03 & 0.81 & 1.32 & 0.826 \\
\hline 3 & $9.7 \%$ & $9.2 \%$ & 0.696 & 0.94 & 0.70 & 1.27 & 0.713 \\
\hline 4 & $56.5 \%$ & $58.0 \%$ & 0.497 & 1.06 & 0.89 & 1.26 & 0.859 \\
\hline \multicolumn{8}{|l|}{ HLA-DQA1 } \\
\hline 1 & $41.8 \%$ & $43.9 \%$ & 0.331 & 1.09 & 0.92 & 1.29 & 0.892 \\
\hline 2 & $12.5 \%$ & $11.6 \%$ & 0.531 & 0.92 & 0.70 & 1.20 & 0.941 \\
\hline 3 & $14.3 \%$ & $14.6 \%$ & 0.819 & 1.03 & 0.81 & 1.31 & 0.823 \\
\hline 5 & $28.3 \%$ & $27.7 \%$ & 0.771 & 0.97 & 0.80 & 1.18 & 0.926 \\
\hline \multicolumn{8}{|l|}{ HLA-DQB1 } \\
\hline 2 & $19.8 \%$ & $17.9 \%$ & 0.273 & 0.88 & 0.71 & 1.10 & 0.947 \\
\hline 3 & $35.5 \%$ & $36.4 \%$ & 0.669 & 1.04 & 0.87 & 1.24 & 0.927 \\
\hline 5 & $17.4 \%$ & $17.3 \%$ & 0.985 & 1.00 & 0.80 & 1.25 & 0.937 \\
\hline 6 & $24.4 \%$ & $26.5 \%$ & 0.254 & 1.12 & 0.92 & 1.36 & 0.882 \\
\hline \multicolumn{8}{|l|}{ HLA-DRB1 } \\
\hline 1 & $10.5 \%$ & $11.4 \%$ & 0.511 & 1.09 & 0.84 & 1.43 & 0.838 \\
\hline 3 & $11.0 \%$ & $9.5 \%$ & 0.266 & 0.85 & 0.64 & 1.13 & 0.886 \\
\hline 4 & $13.7 \%$ & $13.6 \%$ & 0.932 & 0.99 & 0.77 & 1.27 & 0.727 \\
\hline 7 & $12.4 \%$ & $11.6 \%$ & 0.540 & 0.92 & 0.70 & 1.20 & 0.889 \\
\hline 11 & $14.1 \%$ & $14.1 \%$ & 0.968 & 1.00 & 0.79 & 1.28 & 0.603 \\
\hline 13 & $12.0 \%$ & $14.5 \%$ & 0.092 & 1.23 & 0.97 & 1.58 & 0.822 \\
\hline 15 & $14.0 \%$ & $13.9 \%$ & 0.995 & 1.00 & 0.78 & 1.28 & 0.892 \\
\hline
\end{tabular}

HLA types were imputed at 4-digit resolution for all genotyped subjects. However, for statistical power issues, statistics were performed at 2-digit resolution. A minimal allele frequency of $5 \%$ in seronegative or seropositive subjects was set as inclusion criterion for statistical analysis. Bolded values, $p<0.05$; -, seronegative; +, seropositive; OR, odds ratio; $\mathrm{Cl}$, confidence intervals. 
Table S10. Influenza association with anti-NR1 seropositivity, gender-specific

\begin{tabular}{|c|c|c|c|c|c|c|c|c|c|c|}
\hline \multirow{2}{*}{\multicolumn{2}{|c|}{$\begin{array}{c}\text { Study cohort } \\
\text { anti-NR1 seropositivity }\end{array}$}} & \multirow{2}{*}{$\begin{array}{l}\text { GRAS patients } \\
+\end{array}$} & $\begin{array}{l}\text { Affective disorder } \\
\text { patients }\end{array}$ & GRAS controls & \multicolumn{2}{|c|}{ Parkinson patients } & \multicolumn{2}{|c|}{ Parkinson controls } & \multicolumn{2}{|c|}{ TOTAL } \\
\hline & & & + & + & + & - & + & - & + & - \\
\hline \multirow{3}{*}{ Influenza A (males) } & $\mathrm{N}$ seropositive $(\%)$ & $55(80.9) \quad 427(65.7)$ & $8(72.7) \quad 45(76.3)$ & $68(71.6) \quad 454(66.4)$ & $19(67.9)$ & $85(57.8)$ & $2(100.0)$ & $11(57.9)$ & $152(74.5)$ & $1022(65.6)$ \\
\hline & $p$ (Pearson's chi²) & 0.011 & 0.461 & 0.312 & \multicolumn{2}{|c|}{0.322} & \multicolumn{2}{|c|}{0.243} & \multicolumn{2}{|c|}{0.011} \\
\hline & OR [Cl 95\%] & $2.210[1.182-4.131]$ & $0.830[0.193-3.559]$ & $1.276[0.795-2.048]$ & \multicolumn{2}{|c|}{1.540 [0.653 - 3.632] } & \multicolumn{2}{|c|}{ NA } & \multicolumn{2}{|c|}{$1.536[1.102-2.140]$} \\
\hline \multirow{3}{*}{ Influenza B (males) } & $\mathrm{N}$ seropositive (\%) & $26(38.2) \quad 135(20.8)$ & $3(27.3) \quad 19(32.2)$ & $28(29.5) \quad 146(21.3)$ & $11(39.3)$ & $54(36.7)$ & $0(0)$ & $4(21.1)$ & 68 (33.3) & $358(23.0)$ \\
\hline & $p$ (Pearson's chi²) & 0.001 & 0.746 & 0.075 & \multicolumn{2}{|c|}{0.798} & \multicolumn{2}{|c|}{0.471} & \multicolumn{2}{|c|}{0.001} \\
\hline & OR [CI 95\%] & $2.362[1.398-3.990]$ & 0.789 [0.188 - 3.315] & $1.540[0.955-2.482]$ & \multicolumn{2}{|c|}{$1.114[0.486-2.554]$} & \multicolumn{2}{|c|}{ NA } & \multicolumn{2}{|c|}{$1.677[1.225-2.296]$} \\
\hline \multirow{4}{*}{ Influenza A (females) } & $\mathrm{N}$ seropositive (\%) & $16(66.7) \quad 221(67.2)$ & $8(61.5) \quad 45(71.4)$ & $29(69.0) \quad 310(68.9)$ & $6(85.7)$ & $56(69.1)$ & $4(100.0)$ & $17(60.7)$ & $63(70.0)$ & $649(68.2)$ \\
\hline & $p$ (Pearson's chi²) & 0.959 & 0.48 & 0.983 & 0.3 & & 0.1 & & & 32 \\
\hline & OR [CI 95\%] & $0.977[0.406-2.355]$ & $0.640[0.184-2.220]$ & $1.007[0.508-1.996]$ & $2.679[0.30$ & o - 23.433] & $\mathrm{N}$ & & $1.086[0.6$ & $78-1.739]$ \\
\hline & $\mathrm{N}$ seropositive $(\%)$ & $4(16.7) \quad 67(20.4)$ & $2(15.4) \quad 13(20.6)$ & $9(21.4) \quad 75(16.7)$ & $1(14.3)$ & $25(30.9)$ & $0(0)$ & $4(14.3)$ & $16(17.8)$ & $184(19.3)$ \\
\hline Influenza B (females) & $p$ (Pearson's chi²) & 0.663 & 0.665 & 0.433 & 0.3 & & 0.4 & & & 18 \\
\hline & OR [CI 95\%] & $0.782[0.259-2.365]$ & $0.699[0.138-3.553]$ & 1.364 [0.627 - 2.968] & $0.373[0.0$ & $3-3.266]$ & $\mathrm{N}$ & & $0.901[0.5$ & $13-1.584]$ \\
\hline
\end{tabular}

Bolded values, $p<0.05$; +, anti-NR1 seropositive; -, anti-NR1 seronegative; OR, odds ratio; $\mathrm{Cl}$, confidence intervals 


\section{CURRICULUM VITAE}

\section{Personal data}

Name

Date of birth

Place of birth

Nationality

E-mail

\section{Education}

since September 2009

2009

2005
Martesa Tantra

08.08.1982

Bogor, Indonesia

Indonesian

tantra@em.mpg.de

Doctoral candidate of the PhD program "Systems Neuroscience" at Göttingen Graduate School for Neurosciences, Biophysics and Molecular Biosciences (GGNB), Georg-August University of Göttingen, Germany

Doctoral thesis at Clinical Neuroscience (Prof. Dr. Dr. Hannelore Ehrenreich) of the Max Planck Institute of Experimental Medicine, Göttingen, Germany

Master of Science: Cognitive Science University of Osnabrück, Germany Thesis: Patch recognition performance on fractal images as a function of phase noise and distance

Bachelor of Science: Biology Institute Technology of Bandung, Indonesia

Thesis: Effects of prenatal lead exposure on pre-weaning behavior and physical development of F1 generation of Swiss Webster mice

\section{Areas of Expertise}

Adult and neonatal behavioral characterization of genetic mouse models targeting genes relevant to neuropsychiatric disorders (ASD, schizophrenia and substance use disorders), to develop novel mouse models of neuropsychiatric conditions

\section{Research experience}

2009 - Present

Doctoral student in the division of Clinical Neuroscience of the Max Planck Institute of Experimental Medicine, Göttingen, Germany

- Animal (mice) management and husbandry

- Development and establishment of neonatal behavioral phenotyping of transgenic mice 
- Adult behavioral characterization of transgenic mice

- Development and establishment of a new method to assess social dominance in mice

- Development and establishment of a protocol to induce self-administration of benzodiazepines in mice, followed by assessments of the development of tolerance and physical withdrawal

- Application of pharmacological intervention to induce epileptic seizure and determination of seizure propensity of transgenic mice

- Behavioral manipulation of transgenic mice: application of environmental factors such as social stress, enriched environment, pharmacological agents

- Mice perfusion

- Mice genotyping, DNA extraction, isolation and PCR

- Statistical analyses using SPSS and GraphPad Prism

$2008-2009$

$2007-2008$

Researcher at the Neurobiopsychology Research Group of Institute of Cognitive Science, University of Osnabrück, Germany

- Eye tracking experiments: stimuli preparation, recruitment of subjects, conducting the measurements

- Statistical analyses using MATLAB

Member of the researcher team "Computational Understanding of Geometric Analogy Representation" study project at Institute of Cognitive Science, University of Osnabrück

- Development of a computational model of proportional geometric analogies based on the Gestalt psychology

$2004-2005$

Student research assistant of the Animal Physiology Lab of the Department of Biology, Institute Technology of Bandung, Indonesia

$2003-2004$

Student research assistant of the Animal Development and Structure Lab of the Department of Biology, Institute Technology of Bandung

- Management of laboratory animals

- Mice surgery (anesthesia, organ isolation, histology and pathology)

- Neonatal assessment in mice

2003

Intern in the laboratory of Stella Maris Hospital, Makassar, Indonesia

- Collecting and analyzing clinical specimen (blood, urine, excrement and sputum) from patients

- Sputum analysis for the presence of Myobacterium tubercolosis using the acid-fast staining (the Ziehl-Neelsen stain)

$2002-2003$

Student research assistant of the Biochemistry Lab of the Department of Biology, Institute Technology of Bandung 


\section{Teaching Experience}

$2010-2013$

$2011-2012$

$2009-2012$

$2003-2005$

2002
Lecturer in an interdisciplinary workshop: Translational Neuroscience Block 'Schizophrenia' (Lecture and practical part: Testing schizophrenia-relevant functions in mice), Max Planck Institute of Experimental Medicine, Göttingen, Germany

Supervision of internships of Bachelor/ and Master students

Lecturer in an interdisciplinary workshop: Translational Neuroscience Block 'Multiple Sclerosis' (Lecture and practical part: Testing multiple sclerosis-relevant functions in mice), Max Planck Institute of Experimental Medicine, Göttingen, Germany

Student assistant of the Environmental Science Course of the Department of Biology, Institute Technology of Bandung, Indonesia

Tutor at Sony Sugema College, Bandung, Indonesia: assisting students for the preparation of the state examination for the university entrance 